\title{
Reduced Capacitor Eight Bit SAR Analog to Digital Converter
}

by

Daniel Lanfranconi, B. Eng., P. Eng.

\begin{abstract}
A thesis submitted to
Faculty of Graduate Studies and Research

in partial fulfillment of the requirements for the degree of
\end{abstract}

\section{Master of Applied Science in Electrical and Computer Engineering}

\author{
Ottawa-Carleton Institute for Electrical and Computer \\ Engineering \\ Department of Electronics \\ Carleton University \\ Ottawa Ontario \\ January 2014 \\ (C)Copyright
}

Daniel Lanfranconi, 2014 
The undersigned hereby recommend to the Faculty of Graduate Studies and Research acceptance of the thesis entitled

\section{Reduced Capacitor Eight Bit SAR Analog to Digital Converter}

submitted by Daniel Lanfranconi, B. Eng., P. Eng.

in partial fulfillment of the requirements for the degree

of

Master of Applied Science in Electrical and Computer Engineering

Professor Leonard MacEachern, Ph.D., P. Eng. Associate Chair, Graduate, Department of Electronics Thesis Supervisor

Professor Calvin Plett, Ph.D.

Chairman, Department of Electronics

Ottawa-Carleton Institute for Electrical and Computer Engineering

Department of Electronics

Carleton University

January, 2014 


\section{Abstract}

The 8-bit charge sharing successive approximation register (SAR) analog to digital converter (ADC) developed for this thesis was designed using the 130nm IBM CMRF8SF library.

The charge sharing SAR ADC is a hybrid between the binary weighted SAR ADC and the serial SAR ADC. The charge sharing SAR ADC has less capacitors, and therefore less die area and less leakage than a binary weighted SAR ADC. It's conversion time is less than the serial SAR ADC because it uses a capacitor array with more capacitors than the serial SAR ADC.

The charge sharing SAR ADC's performance for the typical nfet and typical pfet (TT) and $+27^{\circ} \mathrm{C}$ process, voltage, and temperature (PVT) point was simulated. The simulated signal to noise ratio (SNR) was measured to be $49.9 \mathrm{~dB}$. The simulated signal to noise and distortion ratio (SINAD) was measured to be $48.72 \mathrm{~dB}$. The simulated effective number of bits (ENOB) was measured to be 7.8 bits. The simulated total harmonic distortion (THD) was measured to be $-60.3 \mathrm{~dB}$. The simulated spurious free dynamic range (SFDR) was measured to be $60.4 \mathrm{~dB}$. The simulated worst case dynamic nonlinearity, differential non-linearity (DNL) and integral non-linearity (INL) are less than $1 / 2$ least significant bit (LSB). 


\section{Acknowledgments}

I would like to express my since gratitude to my wife, Dr. Marlise Peruzzo dos Santos. Leaving my full time job and coming back to school to complete my Master's degree was a difficult choice for my wife and I. I couldn't have made it through this challenging time without her constant motivation, support, and love.

Three other individuals have also been instrumental in helping and encouraging me. They are Dr. Leonard MacEachern, Mr. Nagui Mikhail, and Mr. Hongtao Wang. They have all provided support in ways that have significantly gone above and beyond. Thank you so very much guys! 


\section{Contents}

List of Tables . . . . . . . . . . . . . . . . . . . 5

List of Figures . . . . . . . . . . . . . . . . . . 7

Glossary . . . . . . . . . . . . . . . . . . . . . . . . . . . 13

\begin{tabular}{lll}
\hline Introduction & 17
\end{tabular}

1.1 Motivation . . . . . . . . . . . . . . . . . . . . . . 17

1.2 Thesis Organization $\ldots \ldots \ldots \ldots \ldots \ldots \ldots$

$\begin{array}{lll}2 & \text { Background } & 19\end{array}$

2.1 Introduction . . . . . . . . . . . . . . . . . . . . . . . 19

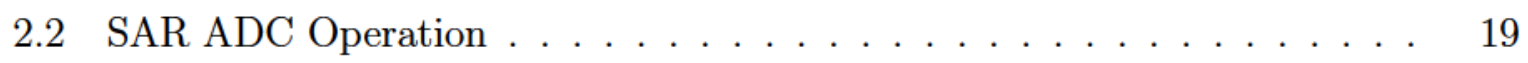

2.2.1 Binary Weighted Successive Approximation Analog to Digital Con-

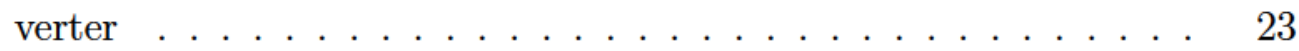

$2.2 .2 \quad$ Serial Successive Approximation Analog to Digital Converter . . . 25

3 Charge Sharing SAR ADC Architecture 29

3.1 Introduction . . . . . . . . . . . . . . . . . . . . . . . . 29

$3.2 \quad$ Charge Sharing SAR ADC $\ldots \ldots \ldots \ldots \ldots$

$3.2 .1 \quad$ Step 1: Initial capacitor array capacitor voltages . . . . . . . . . . 33

$3.2 .2 \quad$ Step $2:$ Generate $\frac{1}{2} V \ldots \ldots \ldots \ldots \ldots \ldots$

$3.2 .3 \quad$ Step $3:$ Generate $\frac{1}{4} V \ldots \ldots \ldots \ldots \ldots \ldots$

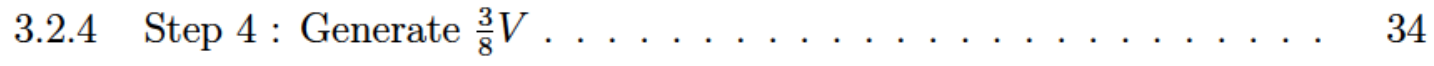

$3.2 .5 \quad$ Step $5:$ Generate $\frac{7}{16} V \ldots \ldots \ldots \ldots \ldots \ldots$

$3.2 .6 \quad$ Step $6:$ Generate $\frac{15}{32} V \ldots \ldots \ldots \ldots \ldots \ldots \ldots$ 
\begin{tabular}{|lll}
4 & Design of Charge Sharing SAR ADC & 37
\end{tabular}

4.1 Introduction . . . . . . . . . . . . . . . . . . . . . 37

$4.2 \quad$ Charge Sharing SAR ADC Design $\ldots \ldots \ldots \ldots \ldots \ldots$

$4.2 .1 \quad$ Sample and Hold Circuit . . . . . . . . . . . . . . . . . 38

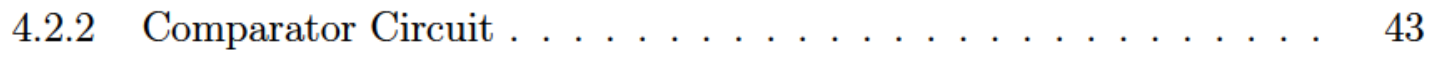

4.2 .2 .1 Clock Inverters A and B . . . . . . . . . 45

$4.2 .2 .2 \quad$ Differential Pair Circuit . . . . . . . . . . . . . 46

$4.2 .2 .3 \quad$ Cross Coupled Inverters $\ldots \ldots \ldots \ldots$

$4.2 .2 .4 \quad$ Negative/Positive Tuning Capacitor Circuits . . . . . . . 48

$4.2 .2 .5 \quad$ Negative/Positive Output Buffer Circuits $\ldots \ldots \ldots . .51$

$4.2 .3 \quad$ Capacitor Array Circuit $\ldots \ldots \ldots$

$4.2 .4 \quad$ SAR Digital Logic Circuit $\ldots \ldots \ldots \ldots \ldots \ldots \ldots$

$4.2 .4 .1 \quad$ CLKINVX1 . . . . . . . . . . . . . . . . . 58

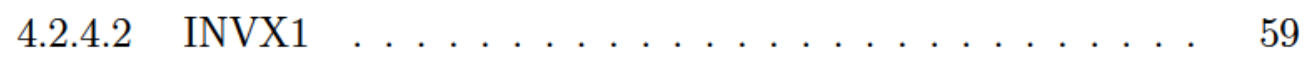

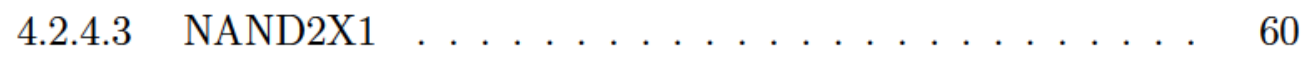

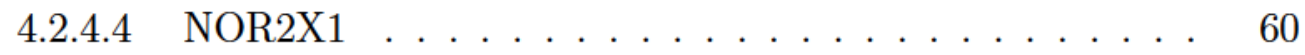

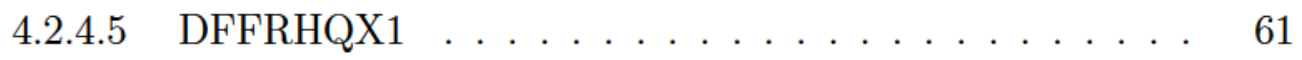

$4.2 .4 .6 \quad D^{2}$

$4.2 .4 .7 \quad$ FILL1 $\ldots \ldots \ldots \ldots \ldots \ldots$

$4.2 .4 .8 \quad$ Verilog RTL $\ldots \ldots \ldots \ldots \ldots$

4.2 .4 .9 Libraries. . . . . . . . . . . . . . . . . . . 70

4.2 .4 .10 Constraints . . . . . . . . . . . . . . . . . . 70

4.2 .4 .10 .1 Cycle to Cycle Jitter . . . . . . . . . . . 70

4.2 .4 .10 .2 Duty Cycle Distortion . . . . . . . . . . 70

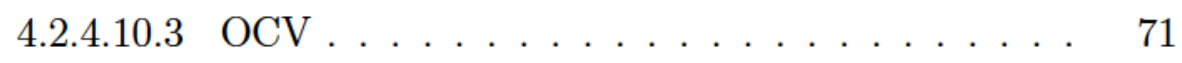

4.2 .4 .10 .4 Allowable Clock Skew . . . . . . . . . . 71

4.2 .4 .11 PNR with Cadence Encounter . . . . . . . . . . . 71

4.2 .4 .11 .1 Sufficient current . . . . . . . . . . . . . . . 75

4.2 .4 .11 .2 Power grid verification . . . . . . . . . . . 75 
4.2.4.11.2.1 $\quad$ Add more power metal . . . . . . . . . 75

4.2.4.11.2.2 Increase the number of VIAs . . . . . . 75

4.2.4.11.2.3 Add decoupling capacitors . . . . . . . 76

$4.2 .5 \quad$ DEF and Verilog import into Virtuoso $\ldots \ldots \ldots$. . . . . . . . . . 76

$4.2 .6 \quad$ Output pad driver $\ldots \ldots \ldots \ldots$

$4.2 .7 \quad$ ESD protection $\ldots \ldots \ldots \ldots$

5 Charge Sharing SAR ADC Simulation Results 81

5.1 Introduction . . . . . . . . . . . . . . . . . . . . . . . 81

5.2 Comparator Monte Carlo Verification . . . . . . . . . . . . . . . . . . . . 81

5.3 Sample and Hold Simulated Performance . . . . . . . . . . . . . . . . . . 91

5.4 SAR Logic and Capacitor Array Verification . . . . . . . . . . . . . . . . 99

5.5 Full Charge Sharing SAR ADC Verification . . . . . . . . . . . . . . 101

5.5 .1 Full Charge Sharing SAR ADC SNR, SINAD, ENOB, THD, and SFDR . . . . . . . . . . . . . . . . . 101

$5.5 .2 \quad$ Full Charge Sharing SAR ADC DNL and INL . . . . . . . . . . . 103

5.6 Leakage Current Simulations $\ldots \ldots \ldots$. . . . . . . . . . . . . . 106

$5.6 .1 \quad$ IBM CMRF8SF FET Drain and Body Leakage Current. . . . . . . 106

\begin{tabular}{|ll|}
\hline Charge Sharing SAR ADC Bench Evaluation & 109
\end{tabular}

6.1 Introduction . . . . . . . . . . . . . . . . . . . . . . 109

6.2 Packaged Die Measurements . . . . . . . . . . . . . . . . . . . . . . . . . 109

6.3 Bare Die Probing Measurements . . . . . . . . . . . . . . . . . . . . . . . 112

6.4 Fibics SEM $\ldots \ldots \ldots \ldots \ldots \ldots$

6.5 Comparison to Published Results . . . . . . . . . . . . . . . . . . 120

\begin{tabular}{lll}
\hline 7 & Conclusion & 123
\end{tabular}

7.1 Contribution to research $\ldots \ldots \ldots \ldots \ldots \ldots$. . . . . . . . . . . . . . . . .

7.2 Future work . . . . . . . . . . . . . . . . . . . . . . . . 125 


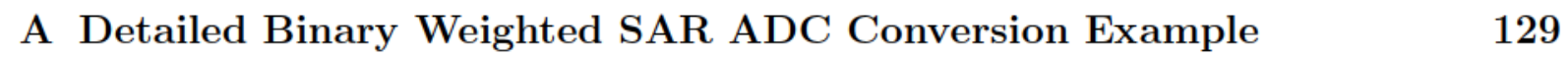

A.1 Step 1: Sampling Mode. . . . . . . . . . . . . . . . . . . . . . . . . . . 130

A.2 Step 2: Hold Mode . . . . . . . . . . . . . . . . . . . . . . . . . 130

A.3 Step 3: Determine binary value of bit $4 \ldots \ldots$. . . . . . . . . . . . . . . 130

A.4 Step 4: Determine binary value of bit $3 \ldots \ldots \ldots$

A.5 Step 5: Determine binary value of bit 2 . . . . . . . . . . . . . . . . . 132

A.6 Step 6: Determine value of bit 1 . . . . . . . . . . . . . . . . . . . . . 133

A.7 Step 7: Determine value of bit 0 . . . . . . . . . . . . . . . 134

B Detailed Serial SAR ADC Conversion Example 137

B.1 Step 1: Determine $\mid$ MSB of $\mid$ ADC|s Digital Output . . . . . . . . . . . . . 138

B.2 Step 2: Determine the Second $\mid$ MSB of $\mid$ ADC/s Digital Output . . . . . . . 138

B.3 Step 3: Determine the Third|MSB of $\mid$ ADC|s Digital Output . . . . . . . 140

B.4 Step 4: Determine the Fourth|MSB of $\mid$ ADC|s Digital Output . . . . . . . . 141

\begin{tabular}{ll|}
\hline C Derivation of SAR Algorithm & 145
\end{tabular}

\begin{tabular}{|l|l|}
\hline D Capacitor Array Voltage Generation Code & 151
\end{tabular}

\begin{tabular}{lll}
\hline E & Derivation of Charge Sharing Equations & 159
\end{tabular}

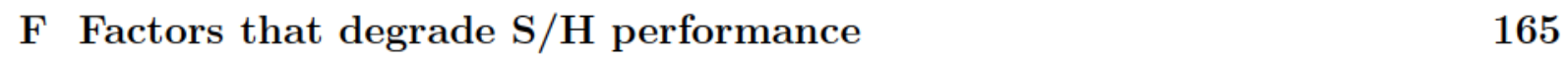

F.1 Charge injection . . . . . . . . . . . . . . . . . . . . 166

F.2 Clock feedthrough $\ldots \ldots \ldots \ldots \ldots$. . . . . . . . . . . . 166

F.3 Conduction . . . . . . . . . . . . . . . . . . . . . . . . . . . . . . 169

F.3.1 Half Conduction . . . . . . . . . . . . . . . . . . . . . 170

F.3.2 Off Region . . . . . . . . . . . . . . . . . . . . . . 173

F.4 Sampling Jitter . . . . . . . . . . . . . . . . . . . . . . . . . . . . . . 174

F.5 Voltage Droop . . . . . . . . . . . . . . . . . . . . . . 174

F.6 Resistance Variation During Track Mode . . . . . . . . . . . . . . . . . . 174 


\section{List of Tables}

2.1 Capacitor values for binary weighted|SAR||ADC, This table describes the association between the capacitors shown in Figure $\mid 2.4$ and the ADC's bit

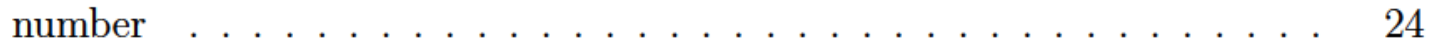

4.1 DFFRHQX1 transistor sizes $\ldots \ldots \ldots \ldots$. . . . . . . . . . . . 63

4.2 DFFSHQX1 transistor sizes . . . . . . . . . . . . . . . . . . 65

\begin{tabular}{|lll|}
\hline 4.3 & Transistor sizes for transistors used by pad driver shown in Figure & 4.31
\end{tabular}$\quad 77$

\begin{tabular}{|lll|l|l|}
\hline 4.4 & Component description used in test bench shown in Figure & 4.32
\end{tabular} . 78

5.1 Description of comparator tests shown in Figure 5.1 . . . . . . . . . . . . 84

5.2 Description of comparator tests shown in Figure 5.2 . . . . . . . . . . . 86

$5.3 \quad$ Summary of simulated $|\mathrm{S} / \mathrm{H}|$ performance. $\ldots \ldots \ldots$. . . . . . . . . . . . 99

5.4 Summary of simulated|ADC|performance. $\ldots \ldots \ldots$. . . . . . . . . . . 103

6.1 Performance comparison between $[1]$ and this work. $\ldots \ldots \ldots$

C.1 All possible charge sharing SAR ADC's capacitor array voltages when $\frac{3}{8} V_{\text {ref }}$ generated. For case of 15 capacitors in the array. . . . . . . . . . . . . . . 147

C.2 All possible charge sharing SAR ADC's capacitor array voltages when $\frac{1}{4} V_{\text {ref }}$ generated. For case of 15 capacitors in the array. . . . . . . . . . . . . . . 147

C.3 All possible charge sharing SAR ADC's capacitor array voltages when $\frac{1}{2} V_{\text {ref }}$ generated. For case of 15 capacitors in the array. . . . . . . . . . . . . . 147 
LIST OF TABLES 


\section{List of Figures}

$2.1 \quad$ Major SAR ADC building blocks. . . . . . . . . . . . . . . 20

$2.2 \quad$ The five voltages generated by the $\mid$ SAR $\mid$ logic in response to the sampled

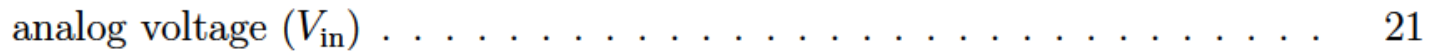

$2.3 \quad$ All possible voltages a five bit|SAR |ADC|s|SAR| logic can generate. . . . 23

2.4 Binary weighted SAR ADC schematic diagram . . . . . . . . . . . 24

$2.5 \quad$ Serial|DAC|schematic diagram showing the circuit configuration when both

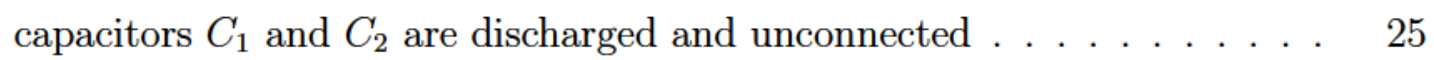

2.6 Major Serial|SAR |ADC|blocks . . . . . . . . . . . . . . . . . . . 27

3.1 Charge sharing $\mid$ SAR $\mid$ ADC|tradeoffs, performance and die area $\ldots . . .30$

3.215 capacitor array schematic diagram showing the circuit configuration when seven capacitors are connected to $V_{\text {ref }}$. . . . . . . . . . . . 33

$3.3 \quad 15$ capacitor array schematic diagram showing the circuit configuration when eight capacitors are connected to ground. The capacitors charged to $V_{\text {ref }}$ in the previous step remain at $V_{\text {ref }} V . \ldots \ldots \ldots \ldots$

3.415 capacitor array schematic diagram showing the circuit configuration when six capacitors are connected in parallel. The voltage across each of these six capacitors becomes $\frac{1}{2} V$. All other capacitors remain at their

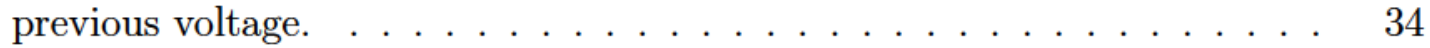

$3.5 \quad 15$ capacitor array schematic diagram showing the circuit configuration when six capacitors are connected in parallel. The voltage across each of these six capacitors becomes $\frac{1}{4} V$. All other capacitors remain at their

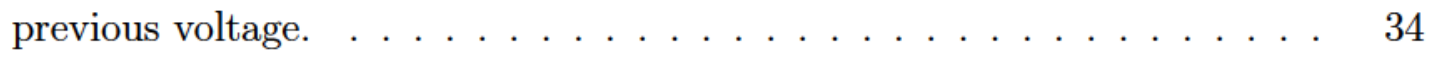


$3.6 \quad 15$ capacitor array schematic diagram showing the circuit configuration when four capacitors are connected in parallel. The voltage across each of these four capacitors becomes $\frac{3}{8} V$. All other capacitors remain at their previous voltage. . . . . . . . . . . . . . . . . . . . . . . 35

$3.7 \quad 15$ capacitor array schematic diagram showing the circuit configuration when four capacitors are connected in parallel. The voltage across each of these four capacitors becomes $\frac{7}{16} V$. All other capacitors remain at their previous voltage. . . . . . . . . . . . . . . . . . . 35

$3.8 \quad 15$ capacitor array schematic diagram showing the circuit configuration when four capacitors are connected in parallel. The voltage across each of these four capacitors becomes $\frac{15}{32} V$. All other capacitors remain at their previous voltage. . . . . . . . . . . . . . . . . . . 35

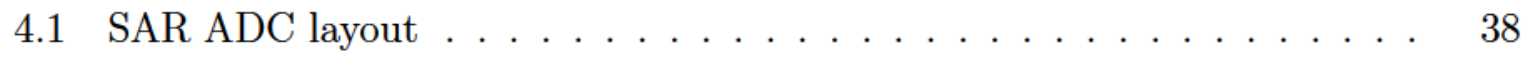

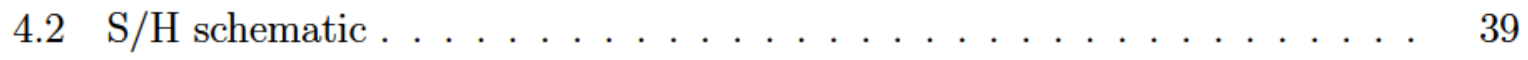

$4.3 \mathrm{~S} / \mathrm{H}$ layout $\ldots \ldots \ldots \ldots \ldots \ldots$

4.4 StrongARM comparator . . . . . . . . . . . . . . . . . . . . 44

4.5 Clock inverter $\mathrm{A}$. . . . . . . . . . . . . . . . . . . . . 46

4.6 Clock inverter B $\ldots \ldots \ldots \ldots \ldots$

4.7 Differential pair transistors . . . . . . . . . . . . . . . . . . 47

4.8 Cross coupled inverters $\ldots \ldots \ldots \ldots \ldots$. . . . . . . . . . . . . . . 48

$4.9 \quad$ Comparator positive output tuning capacitors $\ldots \ldots \ldots$. . . . . . . . . . . 49

4.10 Comparator negative output tuning capacitors . . . . . . . . . . 50

4.11 Comparator negative output buffer schematic . . . . . . . . . . . . . 51

4.12 Comparator positive output buffer schematic . . . . . . . . . . . . . . 52

4.13 Capacitor array layout $\ldots \ldots \ldots \ldots \ldots$. . . . . . . . . . . . 53

4.14 Cross-sectional view of the layer stackup used by the dualmimcap capacitor 53

4.1515 capacitor common centroid diagram . . . . . . . . . . . . . 54

4.16 Example of how dummy capacitors affect etch rate . . . . . . . . . 56

4.17 Example of how the etch rate effects the amount of undercut . . . . . . 56 
4.18 SAR layout $\ldots \ldots \ldots \ldots \ldots \ldots \ldots$. . . . . . . . . . . . . . . . . . . 57

4.19 CLKINVX1 layout $\ldots \ldots \ldots \ldots \ldots$

4.20 INVX1 layout $\ldots \ldots \ldots \ldots$. . . . . . . . . . . . . . . . . . . .

4.21 NAND $2 X 1$ layout $\ldots \ldots \ldots \ldots$. . . . . . . . . . . . . . . . 61

4.22 NOR $2 X 1$ layout $\ldots \ldots \ldots \ldots \ldots \ldots$. . . . . . . . . . . . . . . . . 61

4.23 DFFRHQX1 transistor level schematic . . . . . . . . . . . . . . . . 62

4.24 DFFRHQX1 layout . . . . . . . . . . . . . . . . . . . . . . . . 62

4.25 DFFSHQX1 transistor level schematic . . . . . . . . . . . . . . . 64

4.26 DFFSHQX1 layout . . . . . . . . . . . . . . . . . . . . . . . 64

4.27 FILL1 layout . . . . . . . . . . . . . . . . . . . . . 66

4.28 PNRTFlow $\ldots \ldots \ldots \ldots$

4.29 Mealy and Moore $\mathrm{FSM}$. . . . . . . . . . . . . . . . . . 69

4.30 Register to register timing path $\ldots \ldots \ldots$. . . . . . . . . 73

4.31 Schematic diagram of the pad driver $\ldots \ldots \ldots \ldots$. . . . . . . 77

4.32 Schematic diagram of the pad driver's test bench . . . . . . . . . 78

4.33 Zoomed in view of a single rising edge of the signal applied to the pad driver 79

5.1 Simulated StrongARM comparator analysis. 3.90625mV applied to Vin_n input and $1 \mu V$ applied to Vin_p . . . . . . . . . . . . . . . . . . . . 83

5.2 Simulated StrongARM comparator analysis. 1V applied to Vin_n input and $996.094 \mathrm{mV}$ applied to $V_{i n} \_$p . . . . . . . . . . . . . . 85

5.3 Simulated Monte Carlo StrongARM comparator analysis. $V_{\text {in_n }}$ set to $1 \mu \mathrm{V}$ and $V_{\text {in_p }}$ set to $3.091 \mathrm{mV} \ldots \ldots \ldots \ldots \ldots$. . . . . . . . . . . 87

5.4 Simulated Monte Carlo StrongARM comparator analysis. $\mathrm{V}_{\text {in_n }}$ set to $1 \mathrm{~V}$ and $V_{\text {in_p }}$ set to $\left.0.996094 \mathrm{~V}\right] \ldots \ldots \ldots \ldots$. . . . . . . . . . 88

$5.5 \quad$ Simulated Monte Carlo StrongARM comparator post calibration. . . . . 89

5.6 Simulated Monte Carlo StrongARM comparator offset voltage. . . . . . . 90

5.7 Simulated S/H transient analysis. . . . . . . . . . . . . . . . . . . . 91

$5.8 \quad$ Simulated settling time and hold pedestal $[\mathrm{S} / \mathrm{H} \mid$ transient analysis. . . . . $\quad 92$

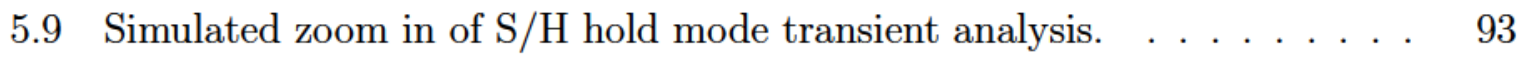


5.10 Hold mode $\mathrm{S} / \mathrm{H}$ transient analysis. . . . . . . . . . . . . . . . 94

5.11 Simulated $\mathrm{S} / \mathrm{H} \mid$ performance $|\mathrm{TT}|+27^{\circ} \mathrm{C} \ldots \ldots \ldots$

5.12 Simulated $|\mathrm{S} / \mathrm{H}|$ performance $|\mathrm{FF}|-40^{\circ} \mathrm{C} \ldots \ldots \ldots$

5.13 Simulated $|\mathrm{S} / \mathrm{H}|$ performance $\mid \mathrm{SS},+125^{\circ} \mathrm{C} \ldots \ldots \ldots$

5.14 SAR/capacitor array transient analysis $\ldots \ldots \ldots \ldots$. . . . . . . . 100

5.15 Simulated $|\mathrm{ADC}|$ performance $\mid \mathrm{TT},+27^{\circ} \mathrm{C} \ldots \ldots \ldots$. . . . . . . . 101

5.16 Simulated $|\mathrm{ADC}|$ performance $|\mathrm{FF}|-40^{\circ} \mathrm{C} \ldots \ldots \ldots \ldots$

5.17 Simulated $\mathrm{ADC}$ performance $\mid \mathrm{SS},+125^{\circ} \mathrm{C} \ldots \ldots \ldots$. . . . . . . . 102

5.18 Simulated $\mid \mathrm{ADC} \| \mathrm{DND}$. . . . . . . . . . . . . . . . . . . . . . . 104

5.19 Simulated $\mid \mathrm{ADC} \| \mathrm{IND}$. . . . . . . . . . . . . . . . . . . . . . . 105

5.20 CMRF8SF pfet transistor leakage test circuit. . . . . . . . . . . . 106

5.21 CMRF8SF pfet transistor source-body and drain-source currents. . . . . 107

5.22 CMRF8SF pfet transistor leakage test circuit. . . . . . . . . . . . . . . 108

5.23 CMRF8SF nfet transistor source-body and drain-source currents. . . . . 108

6.1 Packaged die lab bench evaluation setup . . . . . . . . . . . . . . . . . . 110

6.2 CMC 44 pin clamshell socket and breakout board . . . . . . . . . . . . . 111

6.3 Measured $1 \mathrm{kHz}$ analog input, $1 \mathrm{kHz}|\mathrm{S} / \mathrm{H}|$ clock, $|\mathrm{S} / \mathrm{H}|$ output . . . . . . . . 111

6.4 Lab bench configuration used to probe unpackaged die . . . . . . . . . . 112

6.5 Nine die mounted on conductive plate where one die is probed using six needles . . . . . . . . . . . . . . . . . . . . . . . 113

$6.6 \quad$ Nine die mounted on conductive plate where one die is probed using six needles. View is looking down from above. . . . . . . . . . . . . . . . 113

$6.7 \quad$ Four die mounted on conductive plate . . . . . . . . . . . . . . . 114

6.8 Five hundred times zoom in of die to show "chicken pox". . . . . . . . . 115

6.9 One thousand times zoom in of die to show "chicken pox" more clearly . 115

6.10 Full view of die to show "chicken pox" are present over entire die . . . . 116

6.11 Surface of die captured using SEM $\ldots \ldots \ldots$. . . . . . . . . . . . . . 117

6.12 SEM picture showing "chicken pox" bubble. . . . . . . . . . . . . . . . . 117

6.13 SEM picture of VIA stack . . . . . . . . . . . . . . . . . . . . . 118 
6.14 Fibics Incorporated|FIB|SEM| station. . . . . . . . . . . . . . . . . . . . 119

6.15 SEM image of "chicken pox" bubbles . . . . . . . . . . . . . . . . . . 120

A.1 Binary weighted $S A R|| A D C \mid$ schematic diagram shown in the signal sampling configuration . . . . . . . . . . . . . . . . . . . 130

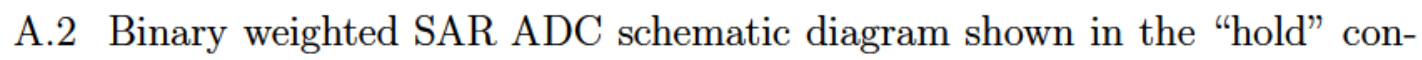
figuration $\ldots \ldots \ldots \ldots \ldots \ldots$. . . . . . . . . . . . . . . . . . . . . . . .

A.3 Binary weighted $\mid$ SAR $\mid$ ADC $\mid$ schematic diagram showing the circuit configuration used to determine the logic value of bit 4 by allowing capacitor "C" to charge . . . . . . . . . . . . . . . . . . . . . . . . . 131

A.4 Binary weighted $\mid$ SAR $\mid$ ADC| schematic diagram showing the circuit configuration used to determine the logic value of bit 3 by allowing capacitor $\frac{\mathrm{C}}{2}$ to charge . . . . . . . . . . . . . . . . . . . . 132

A.5 Binary weighted $|\mathrm{SAR}||\mathrm{ADC}|$ schematic diagram showing the circuit configuration used to determine the logic value of bit 2 by allowing capacitor $\frac{\mathrm{C}}{4}$ to charge . . . . . . . . . . . . . . . . . . 133

A.6 Binary weighted|SAR ||ADC| schematic diagram showing the circuit configuration used to determine the logic value of bit 1 by allowing capacitor $\frac{\mathrm{C}}{8}$ to charge . . . . . . . . . . . . . . . . . . . . . . . 134

A.7 Binary weighted $\mid$ SAR $\mid$ ADC $\mid$ schematic diagram showing the circuit configuration used to determine the logic value of bit 0 by allowing capacitor $\frac{\mathrm{C}}{16}$ to charge.

B.1 Serial|DAC| schematic diagram showing the circuit configuration when capacitor $C_{1}$ is charged to $V_{\text {ref }}$ and $C_{2}$ is discharged to $\left.0 \mathrm{~V}\right]$.

B.2 Serial|DAC schematic diagram showing the circuit configuration when capacitor $C_{1}$ is connected in parallel with capacitor $C_{2}$, charging each capacitor to $\frac{V_{\text {ref }}}{2}$

B.3 Serial|DAC $\mid$ schematic diagram showing the circuit configuration when capacitor $C_{1}$ is charged to $V_{\text {ref }}$ 
B.4 Serial|DAC| schematic diagram showing the circuit configuration when capacitor $C_{1}$ is connected in parallel with capacitor $C_{2}$ to create a voltage across each capacitor of $\frac{3}{4} V_{\mathrm{ref}} \ldots \ldots \ldots$. . . . . . . . . . . . . 140

B.5 Serial|DAC| schematic diagram showing the circuit configuration when capacitor $C_{1}$ is connected to $V_{\text {ref }} \ldots \ldots \ldots \ldots \ldots$. . . . . . . . . 140

B.6 Serial|DAC schematic diagram showing the circuit configuration when capacitors $C_{1}$ and $C_{2}$ are connected in parallel . . . . . . . . . . . . . . . . 141

B.7 Serial|DAC $\mid$ schematic diagram showing the circuit configuration when capacitor $C_{1}$ is connected to ground and $C_{1}$ discharges to $\left.0 \mathrm{~V}\right]$. . . . . . . . 142

B.8 Serial|DAC|schematic diagram showing the circuit configuration when capacitors $C_{1}$ and $C_{2}$ are connected in parallel . . . . . . . . . . . . . . . . 142

B.9 Serial|DAC| schematic diagram showing the circuit configuration when capacitor $C_{1}$ is connected to $V_{\text {ref }} \ldots \ldots \ldots \ldots$. . . . . . . . . . 142

B.10 Serial|DAC schematic diagram showing the circuit configuration when capacitors $C_{1}$ and $C_{2}$ are connected in parallel . . . . . . . . . . . . . . . . 143

B.11 Serial|DAC| schematic diagram showing the circuit configuration when capacitor $C_{1}$ is connected to $V_{\mathrm{ref}} \ldots \ldots \ldots \ldots \ldots$. . . . . . . . . . 143

B.12 Serial|DAC|schematic diagram showing the circuit configuration when capacitors $C_{1}$ and $C_{2}$ are connected in parallel . . . . . . . . . . . . . . . 143

F.1 CMOS transistor drain current f . . . . . . . . . . . . . . . . . . . 167

F.2 CMOS $\mid$ transmission gate drain currents . . . . . . . . . . . . . . . . 167

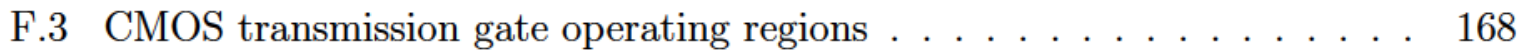




\section{Glossary}

ADC Analog to Digital Converter. 4, 5, 7, 8, 10, 11, 17, 27, 29, 32, 37, 38, 40, 44, 47, [52, 57, 71, 72, 74, 77, 81, 82, 100,106, 110, 112, 120, 123,125, 130,134, 138, 140, 141, 146, 148

CMC Canadian Microelectronics Corporation. 10, 57, 110, 111, 123, 124

CMOS Complementary Metal Oxide Semiconductor. 12, 87, 166,168

CSV Comma Separated Value. 30, 146, 148

DAC Digital to Analog Converter. 7, 11, 12, 25, 27, 138,143

DC Design Compiler. 66, 67, 73

DEF Design Exchange Format. 67, 74, 76

DNL Differential Non-Linearity. 10, 32, 81, 103, 104, 120, 121, 124, 125

DRC Design Rule Check. 66, 67, 72, 76

ECG Electrocardiography. 17, 123

EEG Electroencephalography. 17, 123

EMG Electromyography. 17, 123

ENG Electronystagmography. 17, 123

ENOB Effective Number of Bits. 81, 92, 95, 99, 101, 103, 120, 124 
ESD Electrostatic Discharge. 79, 109

FF Fast nfet, Fast pfet. 10, 97, 99, 102, 103

FFT Fast Fourier Transform. 96, 101

FIB Focused Ion Beam. 11, 119

FSM Finite State Machine. 9, 69

GIDL Gate Induced Drain Leakage. 106108

INL Integral Non-Linearity. 10, 32, 81, $103,105,120,121,124,125$

LSB Least Significant Bit. 27, 82

LVS Layout Versus Schematic. 76

MSB Most Significant Bit. 4, 138 143

OCV On Chip Variation. 70, 71

PCB Printed Circuit Board. 110

PNR Place and Route. 9, 66, 71, 74, 76, 124

PVT Process, Voltage, Temperature. $33,84,86$

RMS Root Mean Squared. 94

RTL Register Transfer Level. 18, 66, 67, 69, 124

S/H Sample and Hold. 5, 8,10, 20, 37, 40, 42, 43, 47, 52, 91, 99, 101, 102, 111, 113, 123 , $124,166,174$

SAR Successive Approximation Register. 5, 7, 11, 17, 27, 29, 32, 37, 38, 40, 44, 47, 52, 57, 71, 72, 74, 76, 77, 81, 82, 99,101, 103, 104, 106, 120, 123, 125, 130,134, 146, 148 
SEM Scanning Electron Microscope. 10, 11, 109, 116,119

SFDR Spurious Free Dynamic Range. 81, 92, 96, 99, 101, 103, 120, 124

SINAD Signal-to-Noise and Distortion Ratio. 81, 92, 94 99, $101,103,124$

SNDR Signal to Noise and Distortion Ratio. 120

SNR Signal-to-Noise Ratio. 81, 92, 94, 96 99, 101,103, 124

SPI Serial Peripheral Interface. 110

SS Slow nfet, Slow pfet. 10, 97, 99, 102, 103

THD Total Harmonic Distortion. 81, 92, 95 99, 101, 103,124

TT Typical nfet, Typical pfet. 10, 97, 99, 101, 103, 124

VNCAP Vertical Natural CAPacitor. 50 


\section{Chapter 1}

\section{Introduction}

\subsection{Motivation}

The key to telemonitoring systems is that they measure signals from patients in every day situations in ways that don't impede a patient's mobility. An accurate diagnosis requires measurement from an unimpeded patient so that the measurements are based on the patient's normal activities. Telemonitoring devices, therefore, must be small and have a long battery life.

Test modalities such as electroencephalography (EEG), electrocardiography (ECG), electromyography (EMG), and electronystagmography (ENG) use signals measured from patients in order to diagnose ailments. The highest frequency these test modalities work in is less than $1 \mathrm{kHz}[2]$. Analog to digital converters are an integral part of a telemonitoring system used by the aforementioned test modalities due to the fact that they convert real world signals into their digital equivalent, which, can be processed and analyzed using modern medical equipment. The SAR ADC is used in bio medical implementations because it provides high energy efficiency [3] [4] [5] [6] at the sampling rates required in these applications. Resolutions of between 6-10 bits are required in these applications due to the fact that higher resolutions waste both power and die area [1] [4]. The SAR ADC is used in applications requiring low power as opposed to integrating and Sigma-Delta ADCs due to the fact that oversampling requirements of Sigma-Delta ADCs and the low 
conversion rate of integrating ADCs have higher energy per conversion cycle than a SAR ADC [7].

One method to reduce the size of a $S \mathrm{SAR} / \mathrm{ADC}$ is to reduce the number of capacitors it uses. Implementing capacitors on a die requires a substantial percentage of the die's area. Therefore, reducing the number of capacitors reduces die size. Capacitors also have leakage currents. So an additional benefit of reducing the number of capacitors also reduces a SAR $\mathrm{ADC}$ s power dissipation and therefore increases battery life.

The objectives of this thesis are to improve upon [1] by reducing the number of capacitors from 28 to 15 in an 8-bit SAR ADC. The measured performance of [1]'s fabricated device was less than simulated due to what [1] commented were "agregious" and "careless layout" issues. Therefore, this thesis will improve upon the fabricated device's performance by using common centroid layout with dummy capacitors.

The contributions to knowledge are an algorithm that controls a 15 capacitor 8-bit SAR ADC and better performance than was measured by [1]. The other contribution is a method to determine which voltages generated by a SAR ADC can be recycled to generate all required voltages - voltages compared to the sampled analog signal.

\subsection{Thesis Organization}

Chap. 2 is a background chapter that describes the binary weighted SAR ADC and the serial SAR ADC. The charge sharing SAR ADC is a hybrid of these two SAR ADC"

Chap. 3 describes the architecture of the charge sharing SAR ADC.

Chap. 4 describes the design of each of the charge sharing SAR ADC]s functional blocks . Chap. 3 also contains sections about Verilog coding styles, and the methodology used to implement the register transfer level (RTL).

Chap. 5 contains the simulation results of each of the charge sharing SAR ADC $\mathrm{s}$ sub-components.

Chap. 6 contains bench evaluation results for the packaged and bare die.

Chap. 7 concludes this work and provides possible techniques for improvement. 


\section{Chapter 2}

\section{Background}

\section{$2.1 \quad$ Introduction}

The operation of the SAR ADC is described in this chapter. The major functional blocks of the SAR ADC are also discussed. An example showing the operation of the $\mathrm{SAR} \mathrm{ADC}$ as it generates the digital equivalent of an analog signal is presented. The digital conversion methods used by the binary weighted SAR ADC and a serial SAR ADC are also presented.

\subsection{SAR ADC Operation}

The SAR ADC generates a digital value that represents the amplitude of a sampled analog waveform. The SAR ADC generates this digital value by successively comparing the sampled analog waveform to a specific number of static voltages generated by the SAR logic. 
The SAR ADC is comprised of three major blocks, sample and hold $(\mathrm{S} / \mathrm{H}), \mathrm{SAR}$ logic, and a comparator. These blocks, and their connections are shown in Figure 2.1.

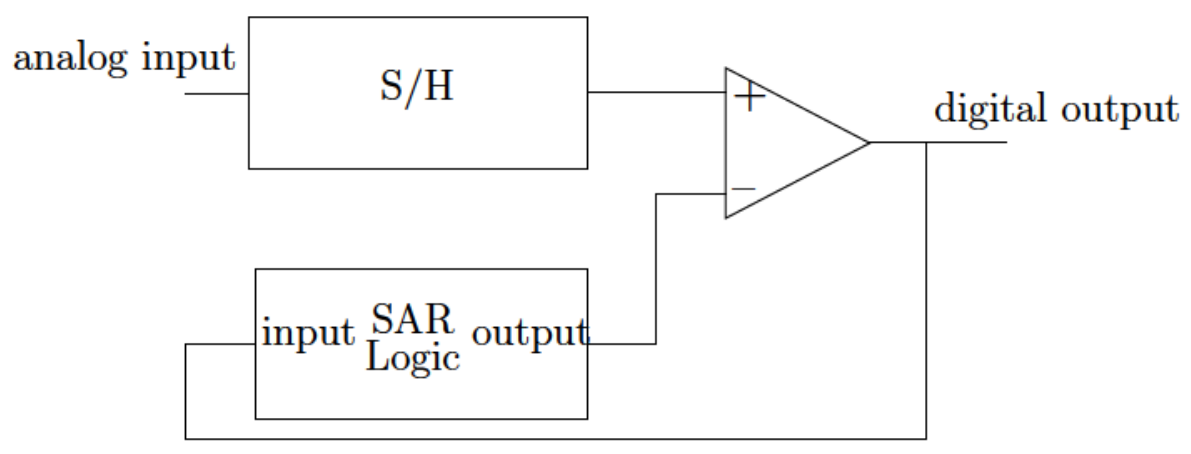

Figure 2.1: Major SAR ADC building blocks.

The $\mathrm{S} / \mathrm{H}$ samples the analog signal applied to the ADC's input. The comparator compares the output of the $\mathrm{S} / \mathrm{H}$ to the output of the SAR logic. A digital logic one is generated by the comparator when the output of the $\mathrm{S} / \mathrm{H}]$ is greater than the voltage generated by the SAR logic. A logic zero is generated by the comparator otherwise. The SAR logic implements an algorithm (implemented in hardware via a custom library of standard cell logic gates) that determines the amplitude of the next voltage to generate based on the result of a comparison between the amplitude of its currently generated voltage and the amplitude of the sampled signal.

The SAR ADC s resolution, or number of bits, defines the number of discrete voltage values that are compared to the sampled analog waveform. For example, a five bit SAR ADC has a resolution of five bits. The five bit SAR compares the sampled analog waveform to five different discrete voltages generated by the SAR logic in order to determine the logical value of its five bits. An example of five voltages generated by the SAR logic, in response to the sampled analog signal, are shown in Figure 2.2 . 


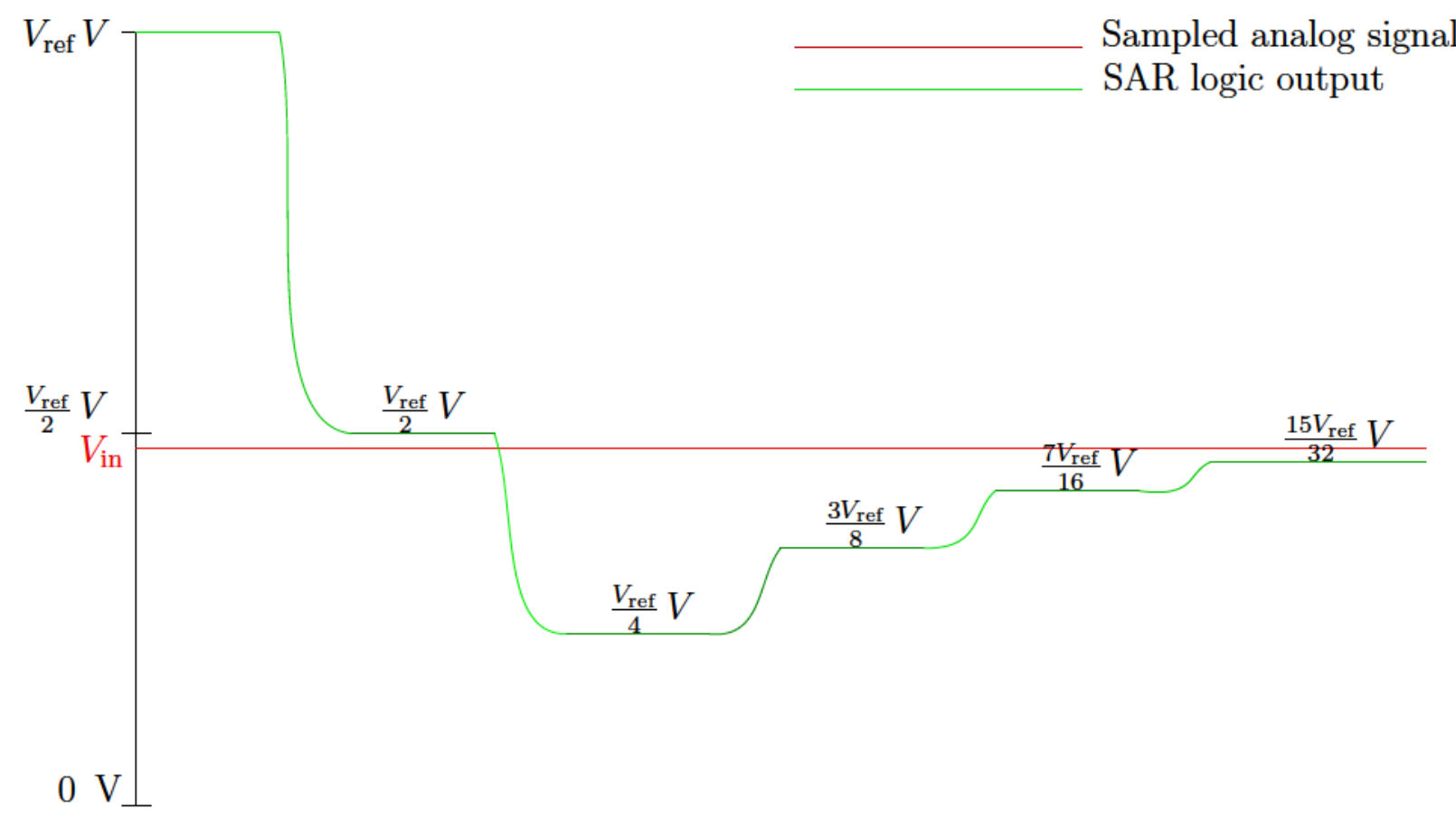

Figure 2.2: The five voltages generated by the $\mathrm{SAR}$ logic in response to the sampled analog voltage $\left(V_{\text {in }}\right)$. The first voltage generated by the SAR logic is always $\frac{V_{\text {ref }}}{2} V$. In this example, the sampled signal voltage is less than $\frac{V_{\text {ref }}}{2} V$ and greater than $\frac{15 V_{\text {ref }}}{32} V$.

The first voltage generated by the SAR logic is one half of the SAR ADC's reference voltage. The SAR ADC s comparator generates a logic zero because the sampled analog signal (denoted as $V_{\text {in }}$ in Figure 2.2) is less than one half of the SAR ADC's reference voltage. Therefore, in this example, the SAR ADC sets its most significant bit, bit 4, to a binary zero value. Next, the SAR ADC compares one quarter of the reference voltage to the sampled analog voltage in the SAR ADC s determination of bit 3's logic value. Three eighths of the reference voltage is compared when determining the logic value of bit 2 , seven sixteenths of the reference voltage when determining the logic value of bit 1 , and fifteen thirty seconds of the reference voltage when determining the logic value of bit 0 . The sampled analog signal is greater than each of the voltages generated by the SAR logic for bits $3,2,1$, and 0 . The resulting digital value generated by the SAR ADC is 01111. Equation 2.1 is used to determine the equivalent analog voltage of this digital 
value,

$$
V_{\text {equivalent }}=V_{\mathrm{lsb}}\left[b_{4}\left(2^{4}\right)+b_{3}\left(2^{3}\right)+b_{2}\left(2^{2}\right)+b_{1}\left(2^{1}\right)+b_{0}\left(2^{0}\right)\right]
$$

where, $V_{\text {lsb }}$ is determined using Equation 2.2.

$$
V_{\mathrm{lsb}}=\frac{V_{\mathrm{ref}}}{2^{\mathrm{N}}}
$$

Equation 2.2 is evaluated to be,

$V_{\mathrm{lsb}}=\frac{1}{2^{5}}=31.25 \mathrm{mV}$.

The equivalent analog voltage of the digital value output by the ADC is determined to be (using Equation 2.1),

$V_{\text {equivalent }}=31.25 m V\left[0\left(2^{4}\right)+1\left(2^{3}\right)+1\left(2^{2}\right)+1\left(2^{1}\right)+1\left(2^{0}\right)\right]=0.46875 V$.

Fig. (2.2) shows all possible voltages that the five bit SAR ADC's SAR logic can generate for comparison with the sampled analog signal. The SAR ADC decides which voltage to generate next based on the current voltage generated by the SAR logic and also based on the binary value output by the SAR ADC's comparator. The comparator generates a logic zero when the voltage generated by the SAR logic is greater than the amplitude of the sampled analog signal. The comparator will generate a logic one otherwise. The next voltage generated by the SAR logic is greater than its current voltage when the comparator's output is a logic one. The next voltage generated by the SAR logic will be less than its current voltage otherwise.

The classic paper [8] introduced the now common implementation of the binary weighted capacitor array SAR ADC, A second classic paper [9], which was published in the same journal, describes an alternate SAR ADC implementation known as the serial SAR ADC. The serial SAR ADC implementation uses significantly less capacitors than the binary weighted capacitor array implementation, described in [8], but unfortunately has the disadvantage of a longer conversion time. The SAR ADC implemented 


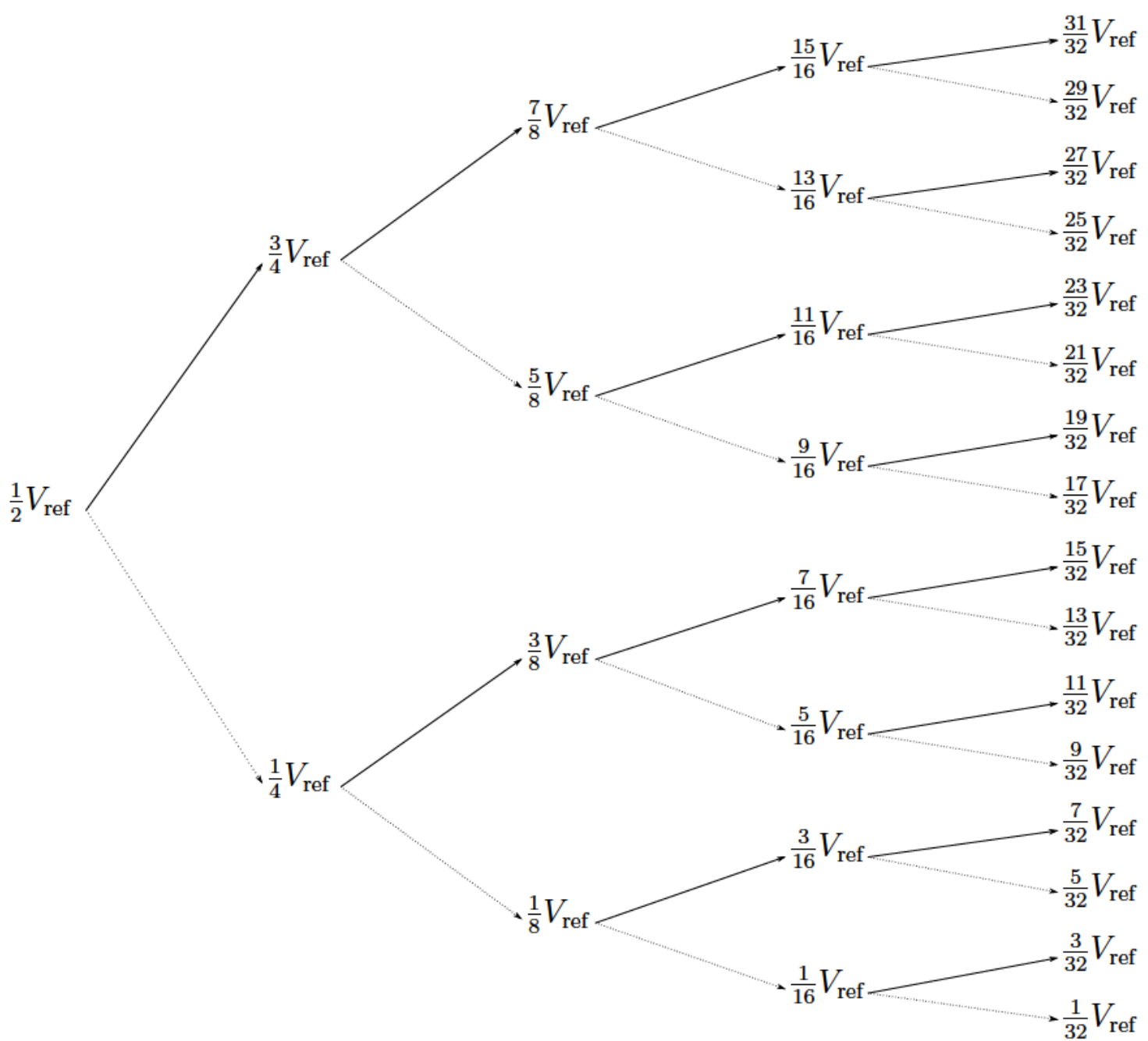

Figure 2.3: All possible voltages a five bit $\mathrm{SAR}$ ADC/s $\mathrm{SAR}$ logic can generate. The next voltage generated by the SAR logic will be one of the voltages shown in this figure. The next voltage will either be greater or less than the current voltage generated by the SAR logic. The decision of which voltage to generate next is based upon if the sampled voltage is less than (shown as a dotted line in the figure) or greater than (shown as a solid line in the figure) the current voltage generated by the SAR logic.

in this work is known as a charge sharing SAR ADC. The charge sharing SAR ADC balances the implementation ideas of both [8] and [9] to balance the number of capacitors in the array with conversion speed.

\subsubsection{Binary Weighted Successive Approximation Analog to Dig- ital Converter}

The binary weighted SAR ADC was introduced by [8]. This ADC is called binary weighted because its operation is based on a specific number of capacitors that are sized based on the binary bit they represent. A schematic diagram of the binary weighted SAR ADC is shown in Figure 2.4. 


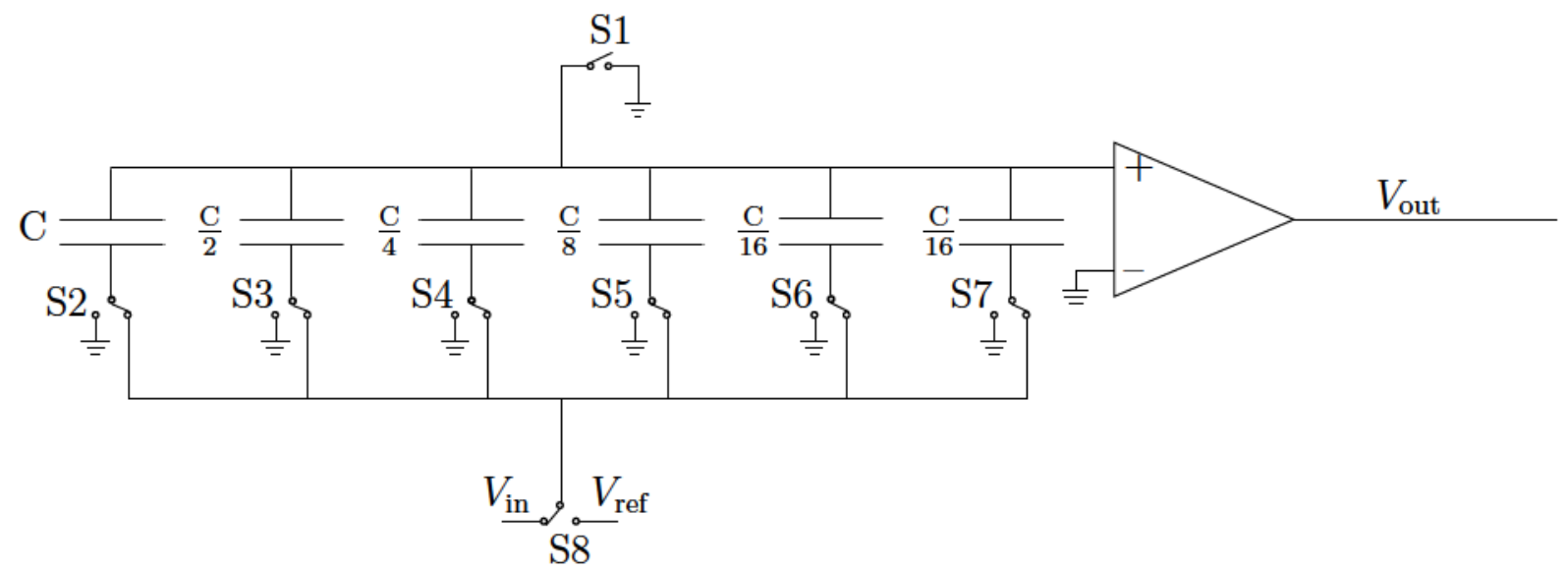

Figure 2.4: Binary weighted SAR ADC schematic diagram. Switch S1 is used to initalize the top plate of the capacitors in the capacitor array. Switches S2, S3, S4, S5, S6, and S7 are used to either initalize the bottom plates of the capacitors in the capacitor array or allow these capacitors to be connected to either $V_{\text {in }}$ or $V_{\text {ref }}$. Table 2.1 describes the association between the capacitors shown in this figure and the ADC's bit number.

\begin{tabular}{|c|c|c|}
\hline Bit Number & Capacitor Size & Switch Number \\
\hline 4 & $\mathrm{C}$ & $\mathrm{S} 2$ \\
\hline 3 & $\frac{C}{2}$ & $\mathrm{~S} 3$ \\
\hline 2 & $\frac{C}{4}$ & $\mathrm{~S} 4$ \\
\hline 1 & $\frac{C}{8}$ & $\mathrm{~S} 5$ \\
\hline 0 & $\frac{C}{16}$ & $\mathrm{~S} 6$ \\
\hline
\end{tabular}

Table 2.1: Capacitor values for binary weighted SAR ADC. This table describes the association between the capacitors shown in Figure 2.4 and the ADC's bit number. This table also shows the association between the capacitors and the switches shown in Fig. (2.4).

The capacitors shown Table 2.1 are sized using Eq. 2.3,

$$
C_{\text {bit number }}=C\left(\frac{2^{\text {bit number }+1}}{2^{\text {number of bits }}}\right)
$$

where $C_{\text {bit number }}$ is the capacitor associated with a specific SAR ADC binary bit (i.e. capacitor $C_{4}$ is associated with bit 4 ), $\mathrm{C}$ is the largest capacitance value in the capacitor array, bit number is the SAR ADC bit (i.e. bit 4), number of bits is SAR ADC resolution (this is the number of bits in the digital word output by the SAR ADC).

As shown in Figure 2.4 an additional capacitor is included in the array that has a capacitance value equal to the least significant bit. This is the right most capacitor shown 
in the diagram and is associated with switch $\mathrm{S} 7$. The reason for this capacitor is described using an example in Appendix A. This capacitor is connected to the comparator's noninverting input. In this example, the capacitor size is $\frac{\mathrm{C}}{16}$.

The binary weighted SAR ADC determines the digital equivalent value of a sampled analog signal is described in detail using an example in Appendix A.

\subsubsection{Serial Successive Approximation Analog to Digital Con- verter}

The operation of the serial SAR ADC was introduced in 9$]$. This SAR is called a serial SAR ADC because this ADC determines the digital value of a sampled analog signal by first determining the value of bit- $\mathrm{N}$, then bit- $\mathrm{N}$ and bit- $\mathrm{N}-1$, then bit-N and bit-N - 1 and bit-N - 2, and so on until all N-bits have been determined. The advantage of this $\mathrm{SAR}$ ADC is that it it requires much less die area than the binary weighted SAR ADC. The serial SAR ADC/s disadvantage is that its conversion time is much longer than a binary weighted SAR ADC s conversion time.

The heart of the serial SAR ADC is the serial digital to analog converter (DAC), as shown in Figure 2.5.

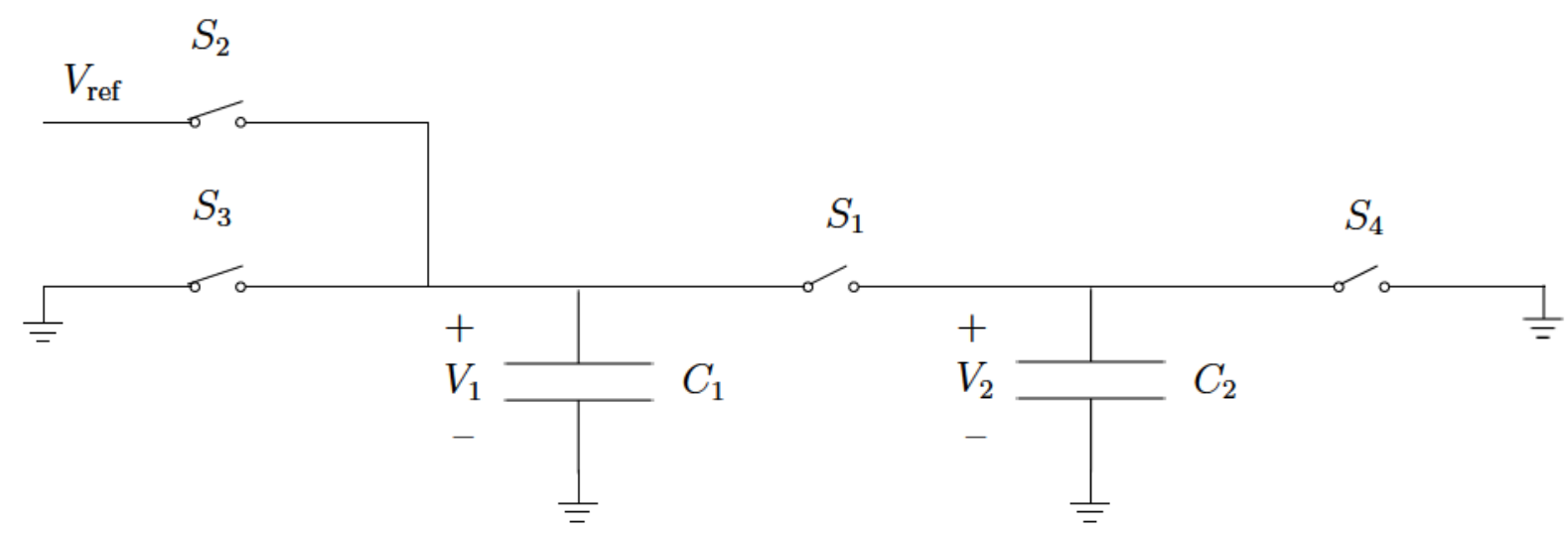

Figure 2.5: Serial DAC schematic diagram showing the circuit configuration when both capacitors $C_{1}$ and $C_{2}$ are discharged and unconnected. Switch $S_{1}$ is used to connect capacitors $C_{1}$ and $C_{2}$ to divide the sum of the voltages across them in half (the capacitance of capacitor $C_{1}$ is equal to the capacitance of capacitor $C_{2}$ ). Switch $S_{2}$ connects capacitor $C_{1}$ 's top plate to $V_{\text {ref. }}$. Switch $S_{3}$ connects capacitor $C_{1}$ 's top plate to ground. Switch $S_{4}$ connects the top plate of capacitor $C_{2}$ to ground.

The serial DAC shown in Figure 2.5 generates analog voltages, as controlled by digital logic in the following two steps. 
1. Close either switch $S_{2}$ to charge capacitor $C_{1}$ to $V_{\text {ref }}$, or, close switch $S_{3}$ to discharge capacitor $C_{1}$ to $0 \mathrm{~V}$. Capacitor $C_{2}$ is discharged to $0 \mathrm{~V}$ when switch $S_{4}$ is closed.

2. Close switch $S_{1}$. Since the value of capacitors $C_{1}$ and $C_{2}$ are the same, the voltage across both capacitors will be the sum of the current voltages across both capacitors divided by two.

Eq. 2.4 is used in [9] to describe the voltage developed across both capacitors after K cycles,

$$
V_{1}(K)=V_{2}(K)=\sum_{i=1}^{K} \frac{2^{i} d_{i}}{2^{K+1}} V_{\text {ref }}
$$

where, $V_{1}(\mathrm{~K})$ is the voltage across capacitor $C_{1}$ after $\mathrm{K}$ cycles, $V_{2}(\mathrm{~K})$ is the voltage across capacitor $C_{2}$ after $\mathrm{K}$ cycles, $d_{i}$ is the logic value of current bit being considered (i.e. if $i=2$ and bit 2 is a logic one, $d_{i}=1$ ), and $V_{\text {ref }}$ is the DAC s reference voltage.

A diagram showing the serial ADC's major building blocks is labeled Figure 2.6.

The analog value generated by the serial $\mathrm{DAC}$ is applied to the inverting input of a comparator and the sampled analog signal (the sampled analog signal is applied to the serial ADC s $V_{\text {in }}$ pin) is applied to the comparator's non-inverting input. The comparator's output is a logic one when the serial DAC s analog voltage is less than the sampled analog signal. Otherwise, the comparator's output is set to a logic 0 .

[9] gives the example of determining the digital equivalent value for the sampled analog signal with amplitude $\frac{13}{16} V_{\text {ref. }}$ This example is described in detail in Appendix B. The example shows that the serial ADC first determines the digital value of the most significant bit, followed by the most significant two bits, followed by the most significant three bits, followed by the most significant four bits.

The general equation describing the number of cycles required to determine the number of cycles for this serial SAR ADC to convert a sampled analog signal into a digital 


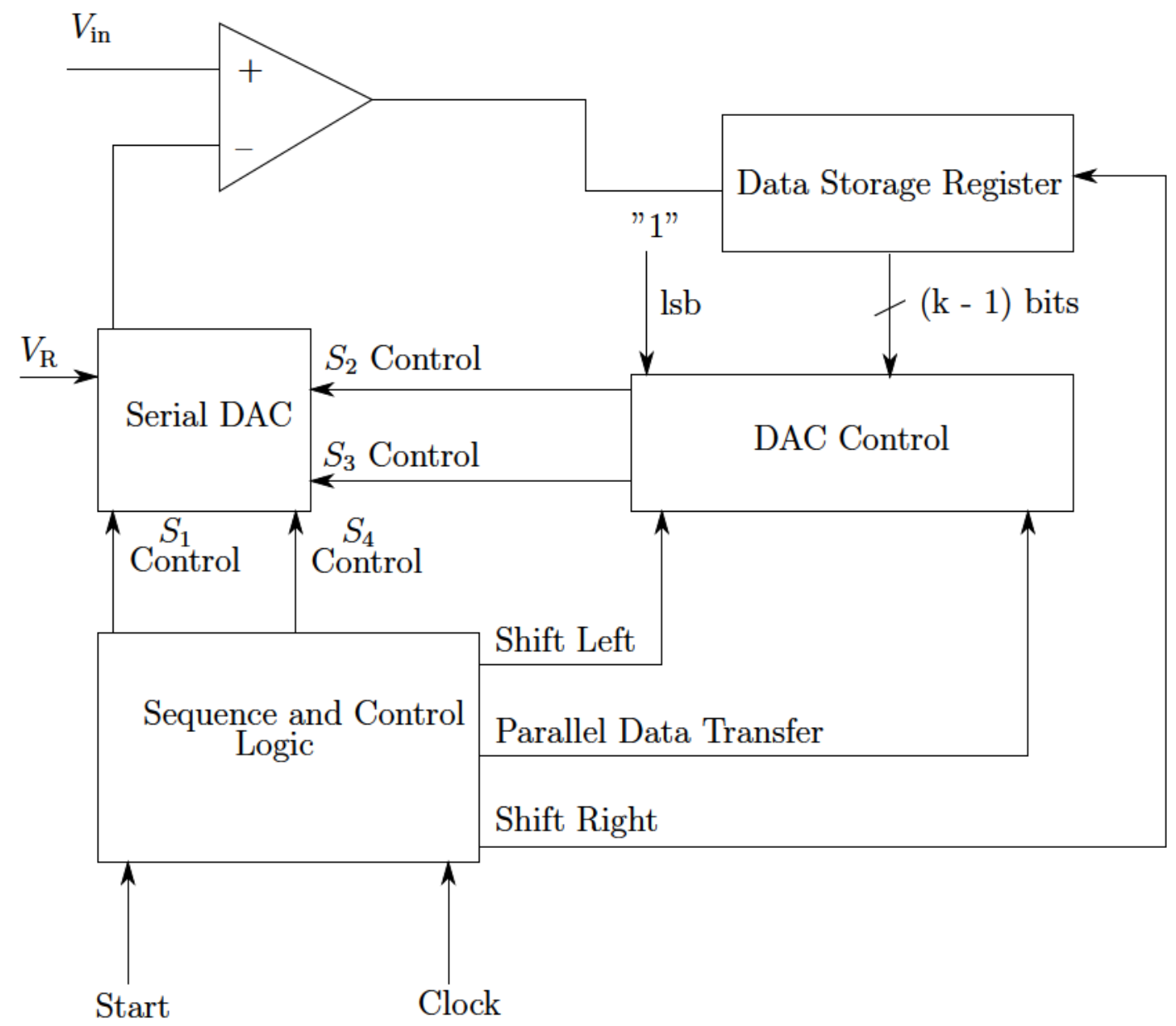

Figure 2.6: Major Serial SAR ADC blocks. The comparator generates a logic one when the sampled analog voltage $\left(V_{\text {in }}\right)$ is greater than the voltage generated by the serial DAC. The data storage block, sets the appropriate bit (the appropriate bit is determined depending on the current phase of the analog to digital conversion) of its data word to the logic state output of the comparator. The data storage block replaces the $\mathrm{LSB}$ of its data word with a logic one and uses this new data word value to determine the state of switches $S_{2}$ and $S_{3}$. The state of switches $S_{1}$ and $S_{4}$ are set based on the sequencing and control logic block. The state of switches $S_{1}, S_{2}, S_{3}, S_{4}$, and the reference voltage (supplied to the serial $\mathrm{DAC}$ by pin $V_{\mathrm{R}}$ ) control the voltage output by the serial DAC.

value is described by Eq. 2.5,

$$
\text { Serial ADC Number Cycles }=N(N+1)
$$

where, $\mathrm{N}$ refers to the number of ADC bits. 


\section{Chapter 3}

\section{Charge Sharing SAR ADC Architecture}

\subsection{Introduction}

This chapter begins with a discussion about how the charge sharing SAR ADC compares to the binary weighted SAR ADC and the serial SAR ADC. The chapter contains a discussion about the required "on" resistance of the charge sharing SAR ADC's transistor switches. An example is used to show the operation of the charge sharing SAR ADC as it generates a digital equivalent value of the sampled analog signal.

\subsection{Charge Sharing SAR ADC}

The two SAR ADC implementations discussed so far can be classified as shown in Figure 3.1 .

The charge sharing SAR ADC lies somewhere between the two SAR ADC $s$ in terms of die size and conversion speed. It requires less die area than the binary weighted SAR ADC and has a faster conversion time than a serial SAR ADC,

The charge sharing SAR|ADC] uses both an algorithm and a capacitor array to convert a sampled analog voltage into an equivalent binary value. In order for the charge sharing ADC to have a faster conversion time than the serial SAR ADC, the charge sharing SAR 


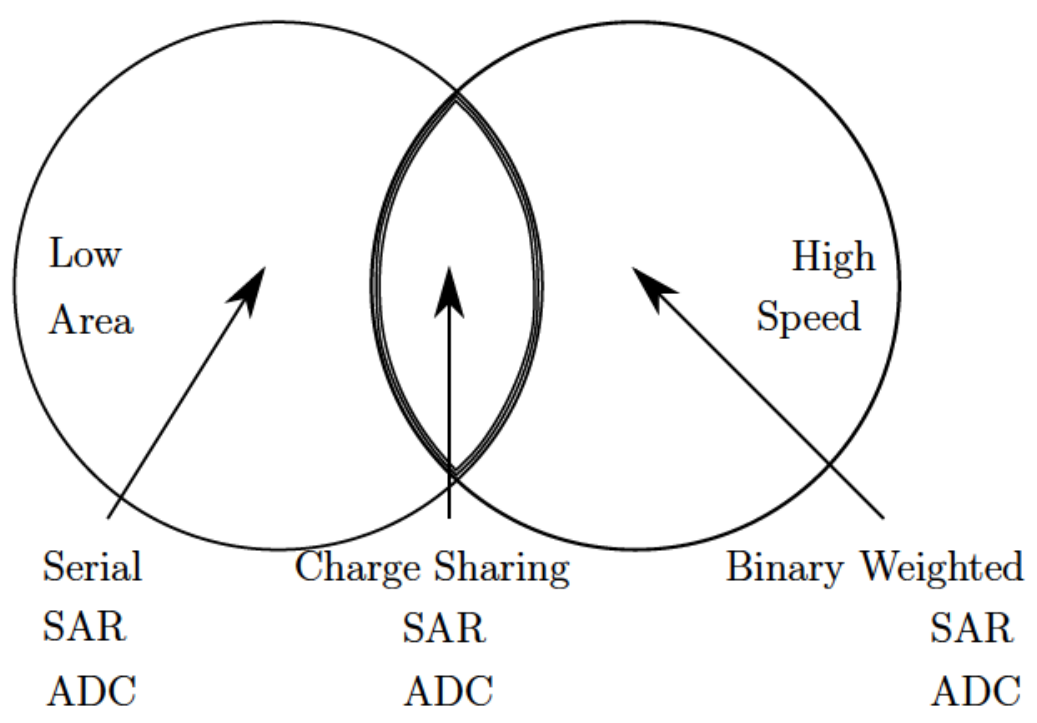

Figure 3.1: Charge sharing SAR ADC tradeoffs, performance and die area. The charge sharing SAR ADC lies somewhere between the binary weighted SAR $\mathrm{ADC}$ in terms of die size and the serial SAR $\overline{A D C}$ in terms of conversion speed.

ADC s capacitor array requires more than two capacitors, but, its capacitor array requires less capacitors than the binary weighted SAR ADC. Its due to the algorithmic nature of the charge sharing SAR ADC that its conversion rate is slower than the binary weighted SAR ADC, Therefore, the number of capacitors and algorithm used by the charge sharing SAR $\mathrm{ADC}$ are the factors that effect conversion time and die area.

A Perl program was developed in order to determine the algorithm as well as the number of capacitors required to implement the 8-bit charge sharing SAR ADC in this thesis. This program generated every possible combination of generated voltages. From this data, an algorithm that traverses the decision tree, such as the tree shown in Figure 2.3 , was developed. To generate the algorithm, the data generated by the Perl program was imported into Excel for analysis via a CSV file. The analysis showed that fifteen unit capacitors were required and the algorithm was developed based on this data. A description of this algorithm is in Appendix D. 
In order for this charge sharing SAR|ADC to generate it's analog voltage, the capacitor array's capacitors are connected (under the control of the algorithm implemented in digital logic) to "share" the voltage stored across each of the connected capacitors. An example of how $\frac{15}{32} V_{\text {ref }}$ is generated by this charge sharing SAR ADC is described in sections 3.2.1, 3.2.2, 3.2.3, 3.2.4, 3.2.5, and 3.2.6.

The capacitors are charged when a transmission gate connects the capacitor to one or more capacitors in the array, the power supply, and/or ground. The transmission gate has a resistance when it is turned on. This resistance is the parallel combination of the pfet's "on" resistance and the nfet's "on" resistance. The transmission gate's resistance decreases as the width of the pfet and/or nfet increase.

Capacitors in the array are charged through the transmission gate and the charging of capacitors in the array can be thought of as a simple RC network. The time to charge one of the array's capacitors can be reduced by reducing the transmission gate's "on" resistance, which is done by increasing the width of the transmission gate's transistors. The series combination of each transmission gate and capacitor pair in the array is in parallel with the other transmission gate and capacitor pairs used to generate voltages. Therefore, assuming that all the capacitors in the array have the same capacitance (all capacitors in the array were laid out using common centroid so that each capacitor in the array is the same) and the transmission gates all have the same "on" resistance means that the charging time of a single transmission gate and capacitor in the array will be the same as all other transmission gates and capacitor pairs in the array.

Giving the capacitor in the array five time constants to be charged, the "on" resistance of its series connected transmission gate is determined using Equation 3.1.

$$
r_{\text {transmission_gate }}=\frac{-t_{\text {charge }}}{C_{\mathrm{p}} \ln \left(\frac{1}{5}\right)}
$$

where, the variables in Equation 3.1 are, $\mathrm{r}_{\text {transmission_gate }}$ is the "on" resistance of the transmission gate, $t_{\text {charge }}$ is the maximum length of time for the capacitor to charge, and $\mathrm{C}_{\mathrm{p}}$ is the capacitance of the capacitor being charged.

The capacitor array capacitors are the minimum capacitance dual MIMCAP capac- 
itors. Two dual MIMCAPs (each $680.17 \mathrm{fF}$ ) were required for each capacitor to be able to lay them out using common centroid layout techniques.

The maximum sampling clock frequency is $20 \mathrm{kHz}(50 \mu$ s period). This ADC was designed with 16 SAR clocks for each sampling clock period. Therefore, the capacitors in the array must be charged within half of the sampling clock frequency multiplied by 16, which is $400 \mu \mathrm{s}$. Therefore, Equation 3.1 was used to determine the maximum "on" resistance of the transmission gate. Equation 3.1 using these values evaluated to be $182 \mathrm{M} \Omega$. If the transmission gate's "on" resistance is larger than this, the capacitors in the array will not be charged quickly enough and the voltage compared to the output of the $\mathrm{S} / \mathrm{H}$ will be incorrect, making the $\mathrm{SAR} A \mathrm{ADC}$ s conversion incorrect.

Increasing $\mathrm{M}_{1}$ and $\mathrm{M}_{2}$ 's width decreases the transmission gate's "on" resistance. However, increasing a transistor's width also increases its input capacitance which increases the amount of time it takes to turn the transistor on [10]. Therefore, simulation is required to ensure $M_{1}$ and $M_{2}$ are sized correctly.

Unfortunately, the capacitors won't all have the same capacitance and the transmission gates won't all have the same "on" resistance due to manufacturing effects. Therefore, there will be differences in the charging times of each transmission gate and capacitor pair in the array and the voltage stored across each capacitor in the array won't be the same. The charging time of the capacitors can vary, as long as each capacitor is completely charged within $50 \mu$ s, the ADC will work correctly. However, the the ADC's [NL and DNL will increase if each capacitor in the array doesn't have the same capacitance.

The design goal is to make the capacitor array as physically small as possible. However, the impact of manufacturing defects increases as the size of a capacitor decreases. Therefore, the capacitors in the array must be made as large as possible to minimize the effect of manufacturing defects balanced with making the capacitors as small as possible so the capacitor array needs the smallest possible area. The minimum area of the capacitor array was very important so it was decided to make each capacitor in the array as small as physically possible $(26.5 \mu \mathrm{m}$ wide and $39 \mu \mathrm{m}$ tall). Therefore, to minimize the effect of manufacturing defects, common centroid layout techniques and dummy capacitors 
were used when laying out the capacitor array. The performance of the capacitor array was verified at the minimum, typical, and maximum $[\mathrm{PVT}]$ points in simulation.

\subsubsection{Step 1: Initial capacitor array capacitor voltages}

The algorithm initializes the fifteen unit capacitors in the array such that seven of the capacitors are charged to $V_{\text {ref, }}$ which in this case is $1 \mathrm{~V}$. The algorithm also discharges the remaining eight capacitors in the array to 0V. This is shown in Figure 3.2 and Figure 3.3 .

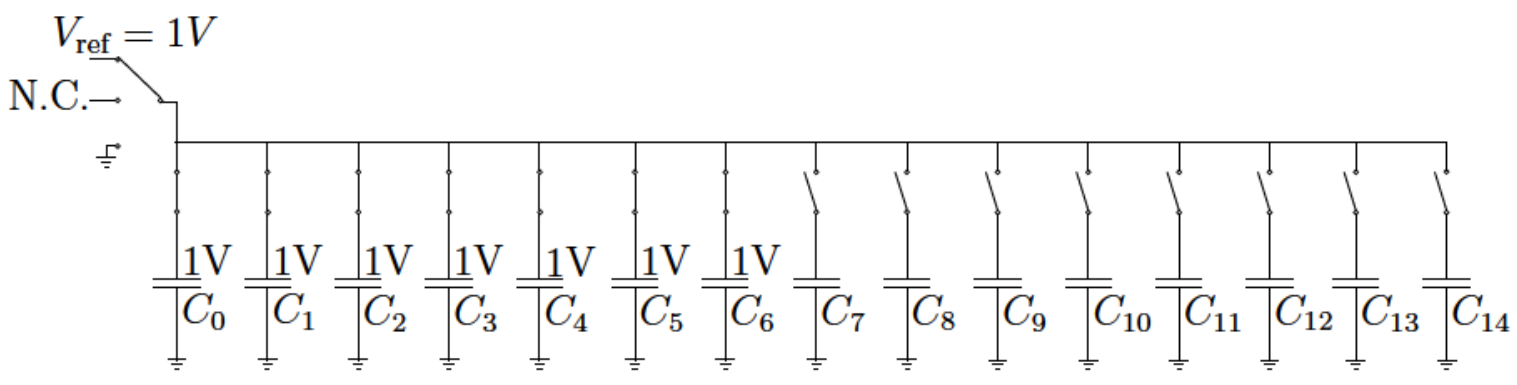

Figure 3.2: 15 capacitor array schematic diagram showing the circuit configuration when seven capacitors are connected to $V_{\text {ref. }}$.

$$
\underbrace{V_{\text {ref }}=1 V}_{\equiv}
$$

Figure 3.3: 15 capacitor array schematic diagram showing the circuit configuration when eight capacitors are connected to ground. The capacitors charged to $V_{\text {ref }}$ in the previous step remain at $V_{\text {ref }} V$.

\subsubsection{Step 2 : Generate $\frac{1}{2} V$}

The algorithm next generates $\frac{1}{2} V$ for comparison to the sampled analog voltage. The capacitor connections are shown in Figure 3.4 .

The algorithm generates $\frac{1}{2} V$ by connecting capacitors $C_{0}, C_{1}, C_{2}, C_{7}, C_{8}$, and $C_{9}$ in parallel and sharing the charge stored on capacitors $C_{0}, C_{1}, C_{2}$ with the uncharged capacitors $C_{7}, C_{8}$, and $C_{9}$.

Appendix $\mathrm{D}$ has a detailed derivation of the equations that describe the voltage across the capacitors in the capacitor array shown in Figure 3.4. 


$$
V_{\text {ref }}=1 \mathrm{~V}
$$

N.C

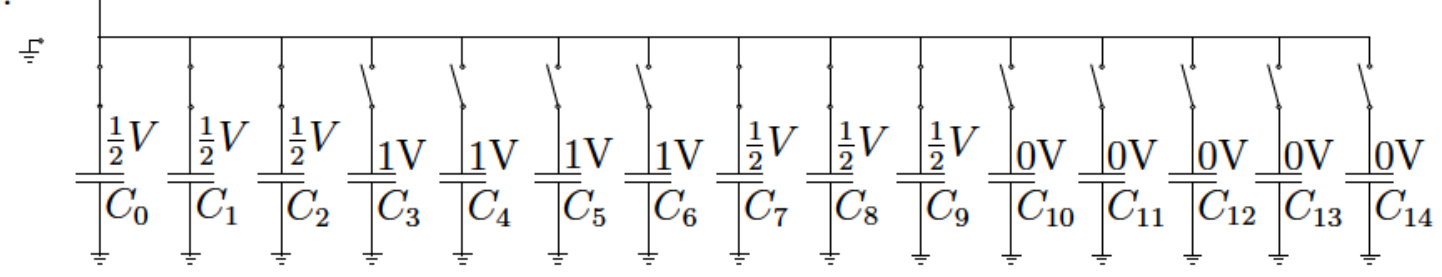

Figure 3.4: 15 capacitor array schematic diagram showing the circuit configuration when six capacitors are connected in parallel. The voltage across each of these six capacitors becomes $\frac{1}{2} V$. All other capacitors remain at their previous voltage.

Figure 3.4 shows that the voltage across capacitors $C_{0}, C_{1}$, and $C_{2}$ equal $1 \mathrm{~V}$ and the voltage across capacitors $C_{7}, C_{8}$, and $C_{9}$ equal $0 \mathrm{~V}$. This simplifies the equations from Appendix D, Equation E.15, Equation E.16, Equation E.17, Equation E.18, Equation E.19, and Equation E.20 so that they all equal $1 / 2 \mathrm{~V}$.

The same analysis can be used to determine the voltage across each capacitor in the capacitor array shown in subsections $3.2 .3,3.2 .4,3.2 .5$, and 3.2 .6 .

\subsubsection{Step 3 : Generate $\frac{1}{4} V$}

The algorithm next generates $\frac{1}{4} V$, which is compared to the sampled analog voltage. The capacitor connections and capacitor voltages are shown in Figure 3.5 .

$$
\begin{aligned}
& V_{\text {ref }}=1 \mathrm{~V} \\
& \text { N.C.- } \\
& \stackrel{5}{=}
\end{aligned}
$$

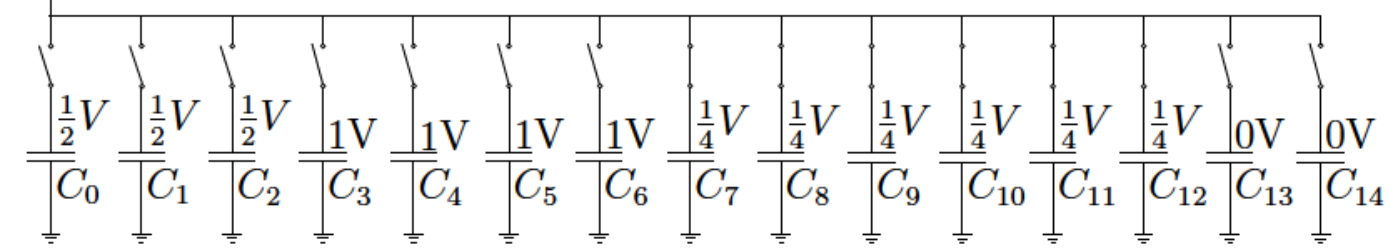

Figure 3.5: 15 capacitor array schematic diagram showing the circuit configuration when six capacitors are connected in parallel. The voltage across each of these six capacitors becomes $\frac{1}{4} V$. All other capacitors remain at their previous voltage.

\subsubsection{Step 4 : Generate $\frac{3}{8} V$}

The third step of this algorithm generates $\frac{3}{8} V$. Then $\frac{3}{8} V$ is compared to the sampled analog voltage. The capacitor connections and capacitor voltages required to generate $\frac{3}{8} V$ is shown in Figure 3.6 . 


$$
V_{\text {ref }}=1 \mathrm{~V}
$$

N.C.

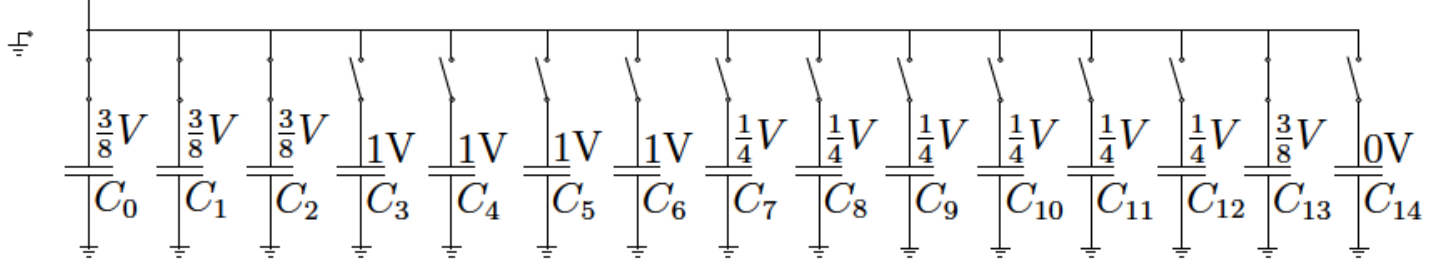

Figure 3.6: 15 capacitor array schematic diagram showing the circuit configuration when four capacitors are connected in parallel. The voltage across each of these four capacitors becomes $\frac{3}{8} V$. All other capacitors remain at their previous voltage.

\subsubsection{Step $5:$ Generate $\frac{7}{16} V$}

The fourth step of this algorithm generates $\frac{7}{16} V$ that is then compared to the sampled analog voltage. The capacitor array configuration required to generate $\frac{7}{16} V$ is shown in Figure 3.7.

$$
V_{\text {ref }}=1 \mathrm{~V}
$$

N.C

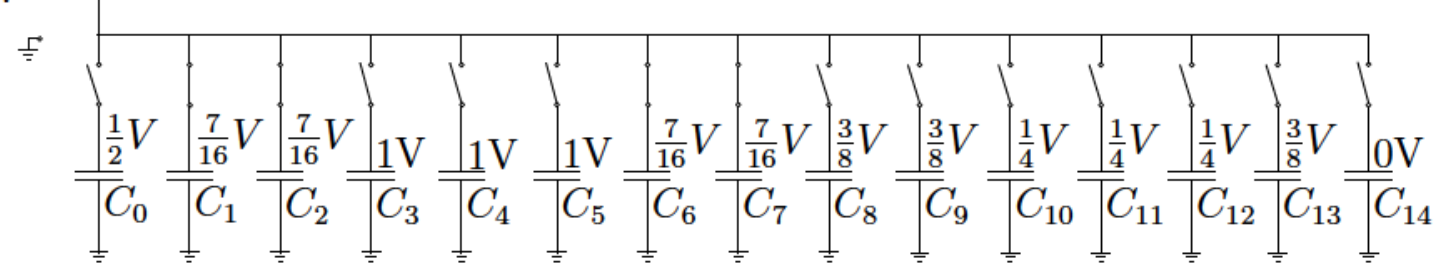

Figure 3.7: 15 capacitor array schematic diagram showing the circuit configuration when four capacitors are connected in parallel. The voltage across each of these four capacitors becomes $\frac{7}{16} V$. All other capacitors remain at their previous voltage.

\subsubsection{Step 6 : Generate $\frac{15}{32} V$}

The next step of this algorithm generates $\frac{15}{32} V \cdot \frac{15}{32} V$ is compared to the sampled analog voltage. Figure 3.8 shows the capacitor array configuration of this step.

$$
V_{\text {ref }}=1 \mathrm{~V}
$$

N.C.

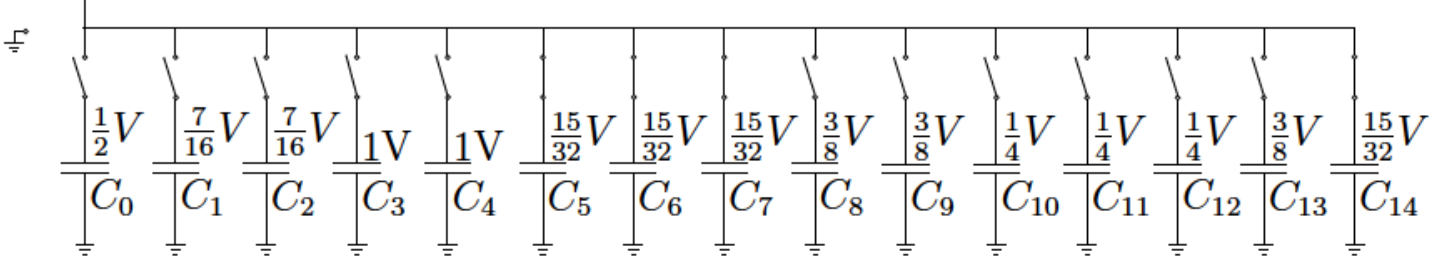

Figure 3.8: 15 capacitor array schematic diagram showing the circuit configuration when four capacitors are connected in parallel. The voltage across each of these four capacitors becomes $\frac{15}{32} \mathrm{~V}$. All other capacitors remain at their previous voltage. 


\section{Chapter 4}

\section{Design of Charge Sharing SAR ADC}

\subsection{Introduction}

This chapter describes the operation and design of the charge sharing SAR ADC's functional blocks. This chapter also describes layout techniques used to improve the performance of the fabricated charge sharing SAR ADC. The chapter describes place and route (PNR) flows and how designs implemented using digital PNR tools can be imported into the Cadence Analog design environment. This chapter also contains methods used to improve electrical parameters such as signal integrity.

\subsection{Charge Sharing SAR ADC Design}

The layout of the charge sharing SAR ADC is shown in Figure 4.1. This charge sharing $\mathrm{SAR}$ ADC $\mathrm{s}$ major building blocks are a $\mathrm{S} / \mathrm{H}$, a comparator, a capacitor array, and a SAR logic block. 


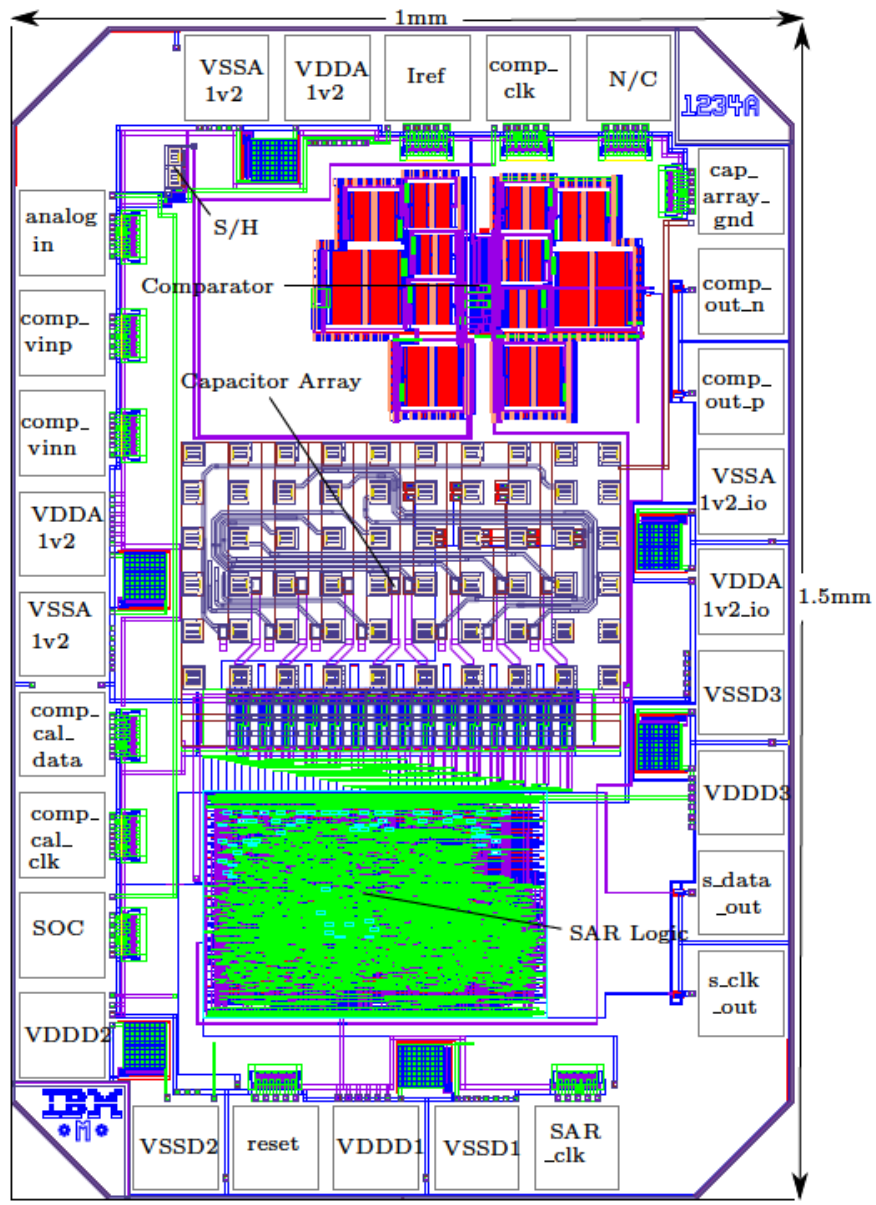

Figure 4.1: $\mathrm{SAR} / \mathrm{ADC}$ layout. The major building blocks of this $\mathrm{ADC}$ are a $\mathrm{S} / \mathrm{H}$, comparator, capacitor array, and SAR Logic. The SAR $\mathrm{ADC}$ layout is $1.5 \mathrm{~mm}$ tall and $1 \mathrm{~mm}$ wide.

\subsubsection{Sample and Hold Circuit}

Figure 4.2 shows the schematic representation of the $\mathrm{S} / \mathrm{H}$ circuit used by the charge sharing SAR ADC. Figure 4.3 shows the layout of the $\mathrm{S} / \mathrm{H}$.

The amplifier was implemented as a common source amplifier operating in the sub threshold region to reduce power consumption. The gain of the amplifier was determined through simulation to be $25 \mathrm{~V} / \mathrm{V}$ in the TT process point, $27^{\circ} \mathrm{C}$. MOSFETs operating in the subthreshold region of operation have a lower current but also have a lower $\mathrm{f}_{\mathrm{t}}$ than when operating in the saturation region [11]. The reduced $\mathrm{f}_{\mathrm{t}}$ isn't a concern due to the low frequency of operation of this device.

The $\mathrm{S} / \mathrm{H}$ uses a switch made of of a single transistor, $\mathrm{M}_{3}$, and a dummy transistor, $\mathrm{M}_{4}$. As transistor $\mathrm{M}_{3}$ turns off, charge used to form its channel flows out of both its 


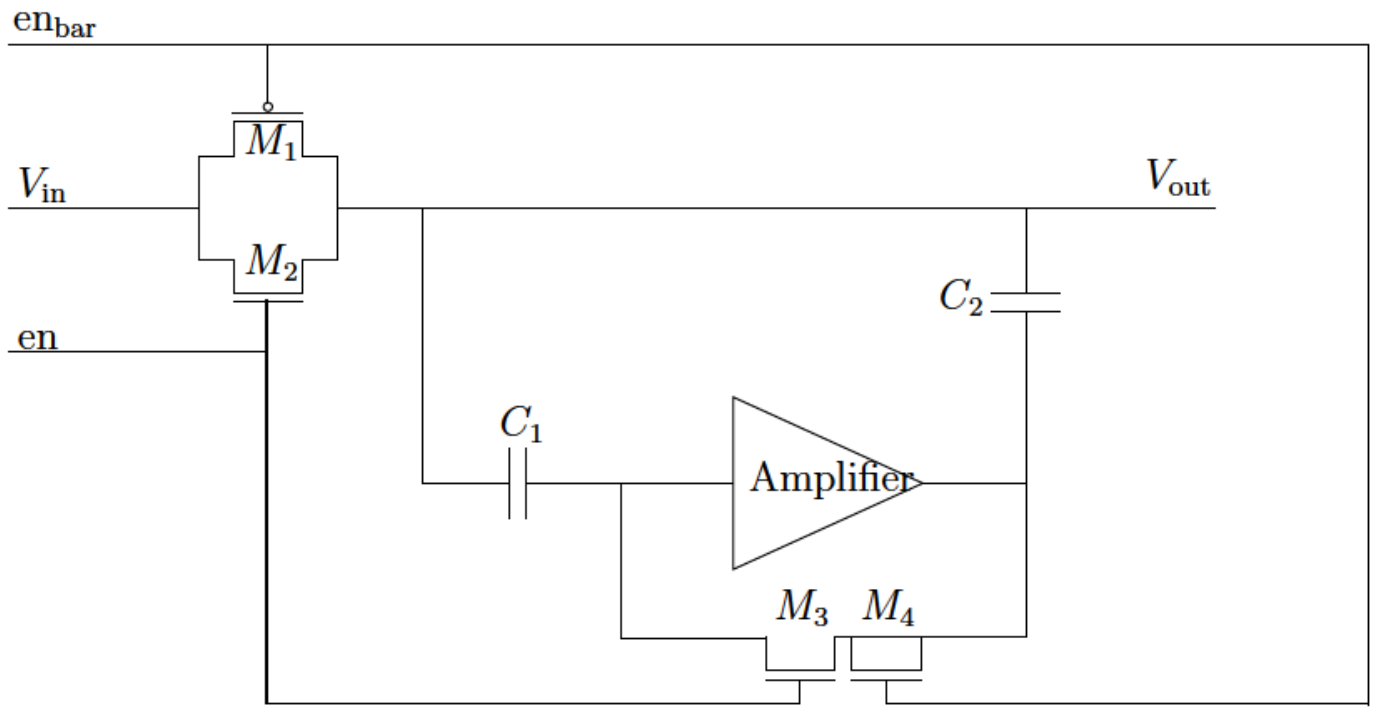

Figure 4.2: $\mathrm{S} / \mathrm{H}]$ schematic. The $\mathrm{S} / \mathrm{H}$ is made up of a transmission gate, amplifier, two capacitors, and a switch that is comprised of a transistor with a series dummy transistor.

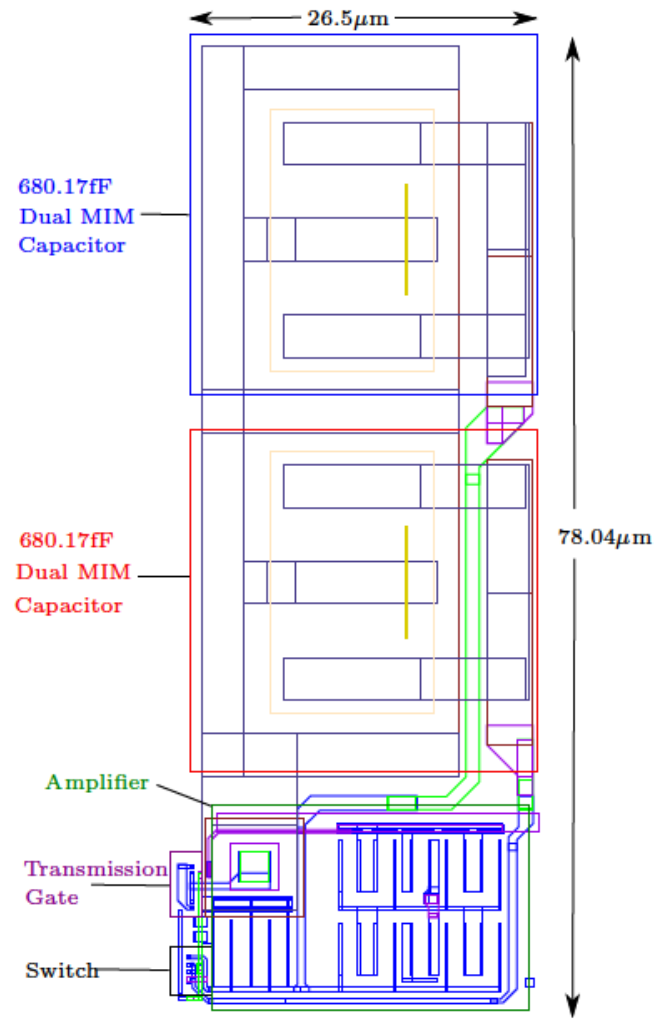

Figure 4.3: $\mathrm{S} / \mathrm{H}$ layout. The $\mathrm{S} / \mathrm{H}$ is made up of two $680.17 \mathrm{fF}$ capacitors, a transmission gate, a switch, and an amplifier. The $\mathrm{S} / \mathrm{H}$ layout is $78.04 \mu \mathrm{m}$ tall and $26.5 \mu \mathrm{m}$ wide.

source and drain terminals. $\mathrm{M}_{4}$ ensures that this charge doesn't affect the charge stored on capacitor $\mathrm{C}_{2}$. This done by turning on $\mathrm{M}_{4}$ as $\mathrm{M}_{3}$ turns off. $\mathrm{M}_{4}$ 's channel is formed using the charge that flows into it from $\mathrm{M}_{3}$. It is commonly accepted that half of $\mathrm{M}_{3}$ 's channel charge flows out of its source and half out of its drain terminals as it turns off. 
Therefore, the width of $\mathrm{M}_{4}$ is half that of $\mathrm{M}_{3}$ 's width. However, there are two situations in which $\mathrm{M}_{4}$ is unable to protect $\mathrm{C}_{2}$ 's charge. The first situation is if more than half of $\mathrm{M}_{3}$ 's charge flows into $\mathrm{M}_{4}$. The excess charge alters the charge stored on $\mathrm{C}_{2}$. The second situation where the charge stored by $\mathrm{C}_{2}$ is altered occurs when $\mathrm{M}_{4}$ isn't turned on at the exact time when $\mathrm{M}_{3}$ turns off.

A transmission gate switch is also used in the $\mathrm{S} / \mathrm{H}$ circuit. The transmission gate switch as shown in Figure 4.2 is made up of transistors $\mathrm{M}_{1}$ and $\mathrm{M}_{2}$.

A transmission gate switch has a lower "on" resistance than if either a single pfet or nfet are used as a switch. The transmission gate exhibits this behavior due to the fact that its "on" resistance is the parallel combination of the pfet's "on" resistance and the nfet's "on" resistance. The transmission gate's resistance decreases as the width of the pfet and/or nfet increase.

Capacitors $\mathrm{C}_{1}$ and $\mathrm{C}_{2}$ are charged through the transmission gate made up of transistors $\mathrm{M}_{1}$ and $\mathrm{M}_{2}$. The parallel combination of capacitors $\mathrm{C}_{1}$ and $\mathrm{C}_{2}$ and the transmission gate's "on" resistance act like a standard RC network. Reducing the transmission gate's "on" resistance has the effect of decreases the time to charge $\mathrm{C}_{1}$ and $\mathrm{C}_{2}$.

Giving $\mathrm{C}_{1}$ and $\mathrm{C}_{2}$ five time constants to be charged, the "on" resistance of the transmission gate formed by $\mathrm{M}_{1}$ and $\mathrm{M}_{2}$ is determined using Equation 3.1 .

Where, the variables in Equation 3.1 are, $\mathrm{r}_{\text {transmission_gate }}$ is the "on" resistance of the transmission gate, $t_{\text {charge }}$ is the maximum length of time for the capacitors $\mathrm{C}_{1}$ and $\mathrm{C}_{2}$ to charge (which is at most, one half of the sampling clock cycle), and $\mathrm{C}_{\mathrm{p}}$ is the parallel combination of capacitors $\mathrm{C}_{1}$ and $\mathrm{C}_{2}$.

The maximum sampling clock frequency is $20 \mathrm{kHz}$. Capacitors $\mathrm{C}_{1}$ and $\mathrm{C}_{2}$ must be charged by the time the $\mathrm{S} / \mathrm{H}$ enters hold mode, which is one half of the sampling period (or $25 \mu \mathrm{s}$ ). The maximum amplitude of the signal applied to the $\mathrm{S} / \mathrm{H}$ is $1 \mathrm{~V}$. Therefore, Equation 3.1 was used to determine "on" resistance of the transmission gate formed using $\mathrm{M}_{1}$ and $\mathrm{M}_{2}$ must be less than $11.42 \mathrm{k} \Omega$. The $\mathrm{S} / \mathrm{H}$ requires that the "on" resistance of the transmission gate be less than $11.42 \mathrm{k} \Omega$. If the transmission gate's "on" resistance is larger than this, the sampled voltage applied to the rest of the SAR ADC s components will be 
incorrect, making the SAR ADC's conversion incorrect. 
The topology used for this $\mathrm{S} / \mathrm{H} \mathrm{I}^{\mathrm{T}}$ is commonly known as a Miller effect $\mathrm{S} / \mathrm{H}$. The Miller effect manifests as an increase in the capacitance between the amplifier's input and output terminals. The effective capacitance is the capacitance of the capacitor used to store the sampled signal, multiplied by the amplifier's gain. This effective capacitance is described by Equation 4.1 [12],

$$
C_{\text {hold-effective }}=(1+A)\left(\frac{C_{1} C_{2}}{C_{1}+C_{2}}\right)
$$

where, $\mathrm{C}_{\text {hold-effective }}$ is the capacitance of the capacitor storing the sampled signal, $\mathrm{A}$ is the gain of the $\mathrm{S} / \mathrm{H}$ s amplifier, $\mathrm{C}_{1}$ is the capacitance value of the capacitor connected to the amplifier's input, and $\mathrm{C}_{2}$ is the capacitance value of the capacitor connected to the amplifier's output.

The $\mathrm{S} / \mathrm{H}$ circuit samples the analog signal applied to its $V_{\text {in }}$ pin when the clock applied

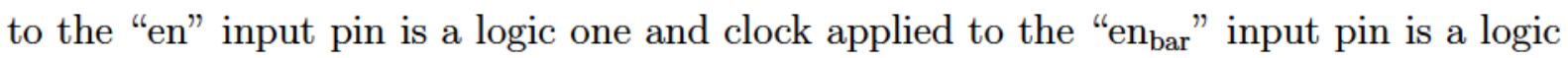
zero. Since the input "en" is a logic high, switch $\mathrm{SW}_{1}$ is enabled, shorting the amplifier and connecting capacitors $\mathrm{C}_{1}$ and $\mathrm{C}_{2}$ in parallel. This is commonly known as "track mode". In this mode, capacitors $\mathrm{C}_{1}$ and $\mathrm{C}_{2}$ store the analog signal applied to the $\mathrm{S} / \mathrm{H} \mathrm{s}$ input.

The $\mathrm{S} / \mathrm{H}$ enters what is commonly known as "hold mode" when the clock transition and the clock applied to the $\mathrm{S} / \mathrm{H}$ 's "en" input is a logic low and clock applied to the

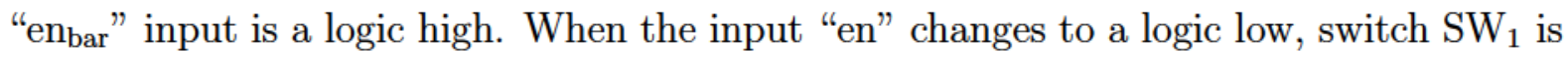
disabled, un-shorting the amplifier and connecting the capacitors in series. In this mode, the analog signal is no longer applied to capacitors $\mathrm{C}_{1}$ and $\mathrm{C}_{2}$ but these capacitors still retain the analog input signal applied to them during "track mode".

$\mathrm{S} / \mathrm{H}$ circuit performance is degraded by several factors. A brief description of these factors are as follows. A more detailed description of these factors is in Appendix F.

- Charge injection : Occurs when a transistor turns off and the charge used to form its

\footnotetext{
${ }^{1}$ The $\mathrm{S} / \mathrm{H}$ implemented by this charge sharing $\mathrm{SAR}$ ADC was designed by Mr. Mark Klibanov, a master's student in the Department of Electronics at Carleton University. The schematic capture, layout, and verification of this $\mathrm{S} / \mathrm{H}$ circuit was performed by Mr. Hongtao Wang, a doctoral student in the Electrical Engineering department at the University of Massachusetts, Amherst.
} 
channel flows out of the transistor's source and drain terminals. This charge distorts the sampled signal by either increasing (for the case of an nfet) or decreasing (for the case of a pfet) the voltage stored on capacitors $\mathrm{C}_{1}$ and $\mathrm{C}_{2}$.

- Clock feedthrough : An error that is due to capacitive coupling to the $\mathrm{S} / \mathrm{H}$ capacitor $\mathrm{C}_{\mathrm{L}}$ from the overlap capacitor $\mathrm{C}_{\mathrm{gd}}$ and the gate capacitor $\mathrm{C}_{\mathrm{ox}}[13$. The amount by which the voltage stored on the $\mathrm{S} / \mathrm{H}$ 's capacitor, $\mathrm{C}_{\mathrm{L}}$, is modified is determined by the difference between the coupled charge and the charge injected by the transistor current.

- Sampling Jitter : Occurs when the $\mathrm{S} / \mathrm{H}$ circuit samples the input analog signal at the incorrect time. This jitter usually manifests itself such that the sampling period isn't consistent and therefore the analog signal isn't sampled at the same point of the sampling clock period. This can lead to aliasing the sampled analog signal.

- Voltage Droop : The amount of voltage discharged from the storage capacitor while the $\mathrm{S} / \mathrm{H}$ circuit is in hold mode.

- Resistance Variation During Track Mode : The $[\mathrm{S} / \mathrm{H}$ s transmission gate switches and the $\mathrm{S} / \mathrm{H}]$ s storage capacitor can be reduced to an $\mathrm{RC}$ network to model the charging and discharging behavior of the S/H's hold capacitor. The varying value of the signal being sampled results in $\mathrm{V}_{\mathrm{gs}}$ of the transmission gate's nfet and pfet transistors to vary. The threshold voltage of the transmission gate will also vary because $\mathrm{V}_{\mathrm{t}}$ is a function of the source-body voltage. The varying value of $\mathrm{V}_{\mathrm{gs}}$ and $\mathrm{V}_{\mathrm{t}}$ result in a variation of the transmission gate's "on" resistance, which, results in a varying charge/discharge time of the $\mathrm{S} / \mathrm{H}$ 's sampling capacitor.

\subsubsection{Comparator Circuit}

Figure 4.4 shows the schematic diagram of the comparator circuit used in the implementation of this charge sharing SAR|ADC. The general operation of the comparator is to compare the amplitude of the analog signal sampled by the $\mathrm{S} / \mathrm{H}$ to the voltage generated by the capacitor array and SAR digital logic. The comparator generates a digital logic 
zero when the sampled analog signal is less than the voltage generated by the capacitor array and SAR digital logic. Otherwise, the comparator generates a digital logic one. This comparison is repeated eight times as this SAR ADC converts an analog signal into an equivalent eight bit digital value.

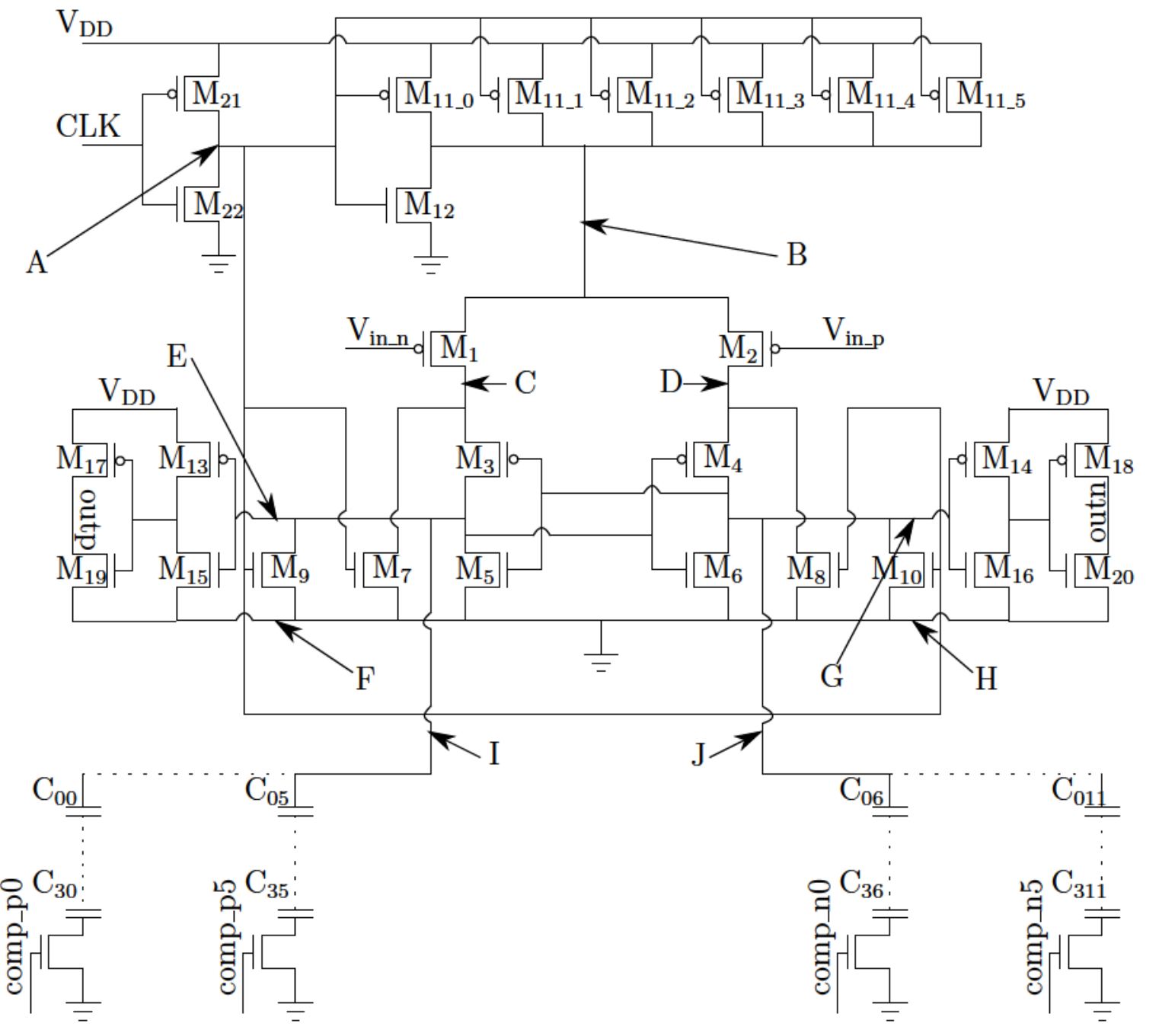

Figure 4.4: StrongARM comparator. The comparator layout is $321.26 \mu \mathrm{m}$ tall and $420.92 \mu \mathrm{m}$ wide.

The comparator implemented in Figure 4.4 is known as a StrongARM comparator [14] [15]. This comparator is a positive, or rising edge triggered, clocked comparator that performs comparisons during the rising edge of the clock input to the comparator.

The clock inverter A circuit generates a logic high when the clock applied to the comparator is a logic low. This results in turning on both the positive shorting transistors and negative shorting transistors, shorting the transistors in the cross coupled inverters so that both the positive and negative outputs of the comparator are logic zero.

The clock inverter B circuit inverters the output of clock inverter A, generating a 
logic low. Resulting in no current flow through both differential pair transistors and also inhibiting current flow through the cross coupled inverters. This reinforces the logic low output by the comparator when the clock input to the comparator is a logic low.

When the clock input to the comparator transitions from a logic low to a logic high, known as the rising edge of the clock, the output of clock inverter A becomes a logic low, turning off both the positive and negative shorting transistors and unshorting the cross coupled inverters. Clock inverter B inverts the output of clock inverter A, generating a logic high and allowing current to flow into the differential pair transistors and also into the cross coupled inverters. The amount of current flowing through each of the differential pair transistors is dependent on the voltage applied to the respective transistor's gate, via the comparator's inputs. The amount of current that flows through each transistor in the differential pair sets the current that flows into each of the cross coupled inverters. Therefore, one inverter will have more current flowing though it than the other inverter. Resulting in the inverter with more current outputting a logic low and the inverter with less current outputting a logic high. Since the inverters are cross coupled, the inverter with less current flowing through it, and a logic high on its output, feeds the input of the inverter with more current flowing though it and a logic low on its output, reinforcing the logic level already achieved by both inverters based on current flow. The result is that the cross coupled inverters reach their logic state very quickly. This can be thought of as the cross coupled inverters having a high gain because they reach a steady logic level very fast and remain at this logic level. This is, of course, until the clock input to the comparator is a logic low, turning on both the positive shorting transistors and negative shorting transistors, forcing both of the comparator's outputs once again to a logic low.

\subsubsection{Clock Inverters A and B}

Figure 4.5 shows a schematic diagram of comparator's clock inverter A. This inverter is connected to the comparator's clock input. Its function is to invert the clock to turn on positive and negative shorting transistors when comparator's clock is a logic low to force the comparator's output to a logic low. 


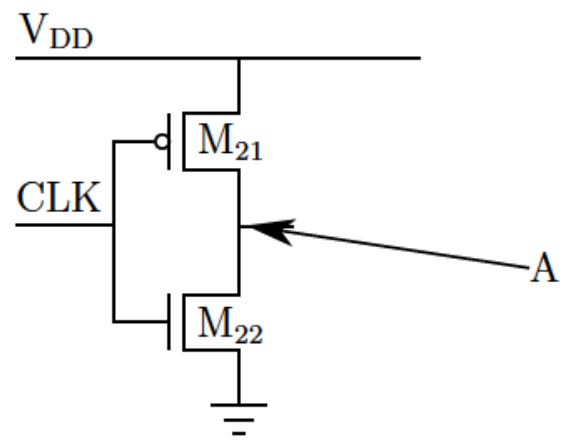

Figure 4.5: Clock inverter A. Transistors $\mathrm{M}_{21}$ and $\mathrm{M}_{22}$ form an inverter that inverts the clock input to the StrongARM comparator. Transistor $\mathrm{M}_{21}$ is $1 \mathrm{um}$ wide, $120 \mathrm{~nm}$ long, and has 1 finger. Transistor $\mathrm{M}_{22}$ is $320 \mathrm{~nm}$ wide, $120 \mathrm{~nm}$ long, and has 1 finger. Label A refers to labelled point A in Figure 4.4.

Figure 4.6 is the schematic diagram of the comparator's clock inverter B. It inverts the output of clock inverter A to shut off the flow of current when the comparator's clock is a logic low. This reinforces the logic low output by the comparator when the comparator's clock is a logic low.

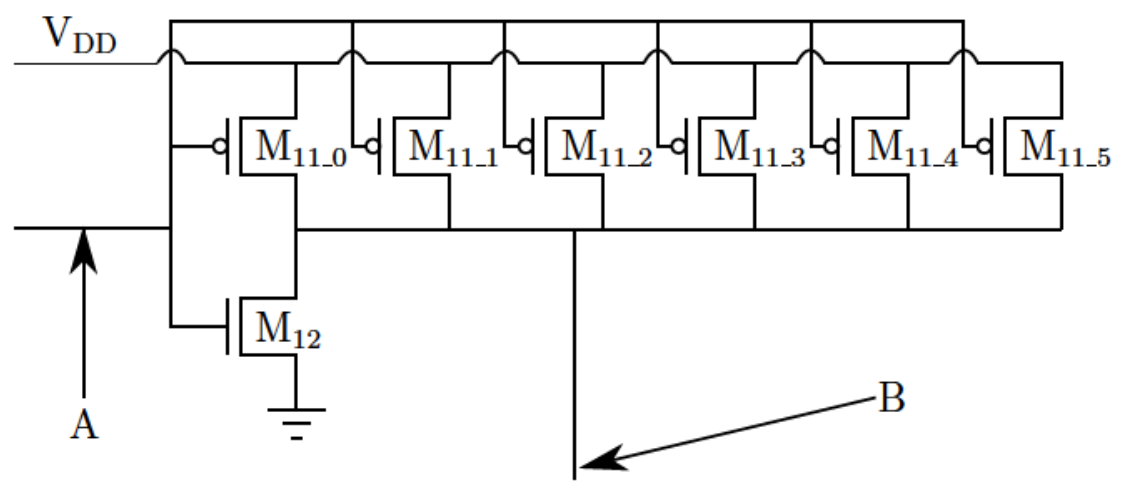

Figure 4.6: Clock inverter $B$. Pfet transistors $M_{11 \_0}, M_{11 \_1}, M_{11_{-} 2}, M_{11_{-} 3}, M_{11_{-} 4}, M_{11 \_5}$ and the nfet transistor $\mathrm{M}_{12}$ form an inverter. These transistors disable current flow into the StrongARM comparator when the clock input to the comparator is low. The six pfet transistors are wired in parallel. These pfets are each $4 u m$ wide, $120 \mathrm{~nm}$ long, and have 1 finger. Nfet transistor, $\mathrm{M}_{12}$ is $2 \mathrm{um}$ wide, $120 \mathrm{~nm}$ long, and has 1 finger. Labels A and B refer to labelled points A and B in Figure 4.4 .

As Figure 4.6 shows, the inverter has six PMOS transistors connected in parallel. This creates an PMOS transistor that is eight times wider. The reason why six transistors are physically connected in parallel instead of using six "fingers" is to more accurately model the transistors in this circuit for Monte Carlo simulations.

\subsubsection{Differential Pair Circuit}

The differential pair transistors are shown in Figure 4.7. The gates of these transistors are connected to the positive and negative inputs of the comparator. The voltage differ- 
ence applied to the gates of these transistors control the current flow difference flowing through these transistors, which in turn, controls the logic level of the output of the cross coupled inverters. The logic level of the cross coupled inverters sets the output of the differential output of the comparator.

When the voltage of $V_{\text {in_n }}$ is greater than $V_{\text {in_p }}$, the current flowing through transistor $M_{1}$ is less than the current flowing through transistor $M_{2}$. This causes the negative output of the comparator to be set to a logic high and the positive output to be set to a logic low. The logic level of the comparator's positive output is a logic high and the comparator's negative output is a logic low in the reverse situation when the voltage applied to $\mathrm{V}_{\text {in_p }}$ is greater than $V_{\text {in_n }}$.

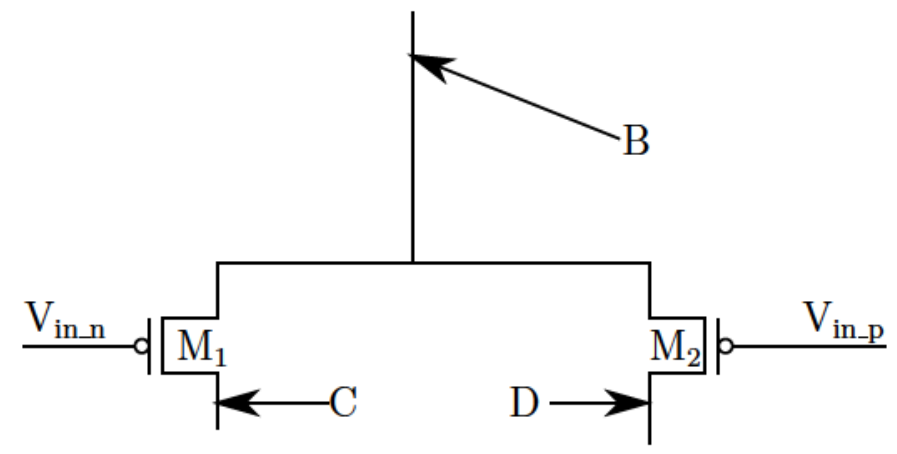

Figure 4.7: Differential pair transistors. Pfet transistors $M_{1}$ and $M_{2}$ form a differential pair circuit. The gates of these transistors are connected to the amplifier's external positive and negative input pins. The difference between the negative and positive inputs, causes a difference in current flow through each of these transistors. Both transistor $\mathrm{M}_{1}$ 's and $\mathrm{M}_{2}$ 's width are each $60 \mathrm{um}$, length are each $120 \mathrm{~nm}$, and each has 1 finger. Labels B, C, and D refer to labelled points B, C, and D in Figure 4.4 .

The operation described above is used to compare the output of the $\mathrm{S} / \mathrm{H}$ to that of the capacitor array/SAR logic to determine if the voltage generated by the capacitor array/SAR logic approximates the sampled signal's voltage level. The comparator generates a logic high when the capacitor array/SAR logic approximates the sampled signal's voltage level. Otherwise, the comparator generates a logic low. This is the basis for how the charge sharing $[\mathrm{SAR} / \mathrm{ADC}$ converts an analog signal into a digital value.

\subsubsection{Cross Coupled Inverters}

Figure 4.8 shows the schematic representation of the cross coupled inverters used by the StrongARM comparator. 


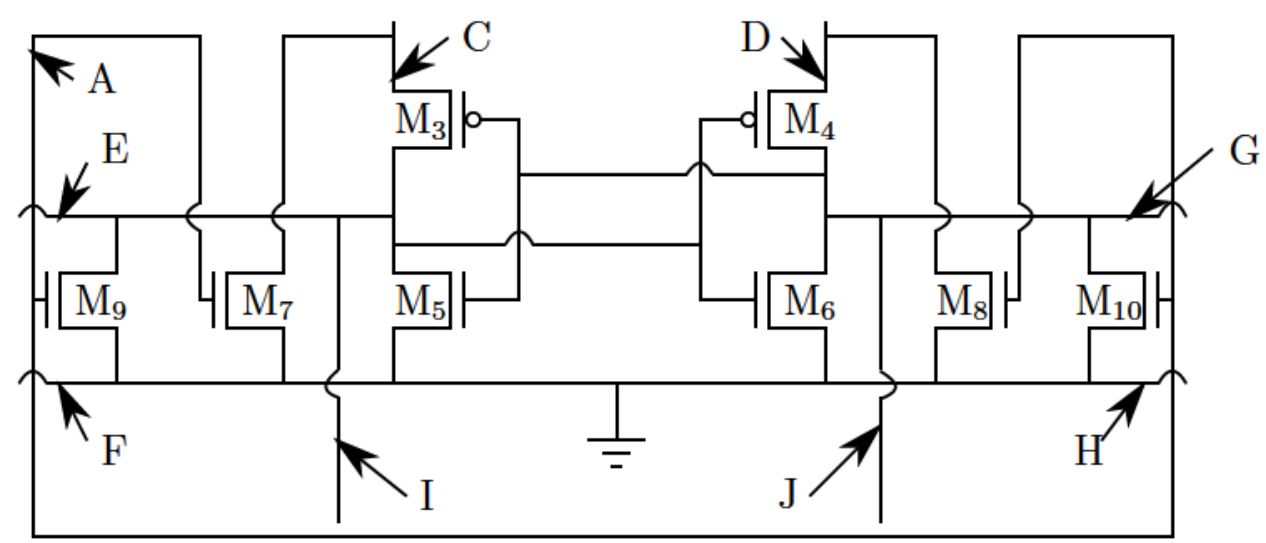

Figure 4.8: Cross coupled inverters. Transistors, $M_{3}$ and $M_{5}$ form one inverter and transistors $M_{4}$ and $\mathrm{M}_{6}$ form a second inverter. The two inverters are cross coupled to form an edge triggered latch that stores the comparator's decision when the comparator's clock input transistions from a low to a high level. Transistors $\mathrm{M}_{3}$ and $\mathrm{M}_{4}$ are each 60um wide, $120 \mathrm{~nm}$ long, and have one finger. Transistors $\mathrm{M}_{5}$ and $\mathrm{M}_{6}$ are each $60 \mathrm{um}$ wide, $120 \mathrm{~nm}$ long, and each have 1 finger. Transistors $\mathrm{M}_{7}, \mathrm{M}_{8}, \mathrm{M}_{9}$, and $\mathrm{M}_{10}$ short out the inverters when the clock input to the comparator is low, forcing both of the comparator's outputs low. Transistors $\mathrm{M}_{7}$ and $\mathrm{M}_{9}$ are known as the "positive shorting transistors". Transistors $\mathrm{M}_{8}$ and $\mathrm{M}_{10}$ are known as the "negative shorting transistors". Labels A, C, D, E, F, G, H, I, and J refer to labelled points A, C, D, E, F, G, H, I, and J in Figure 4.4 .

As previously described, the reason why the inverters are cross coupled is to create a high gain, rising edge clocked storage circuit to store a single digital value. The logic value is determined by these inverters based on the difference in current flowing through the differential pair transistors. The inverter with more current generates a logic low. The inverter with less current generates a logic high. Cross coupling these inverters reinforces their logic level and, in effect, act like a high gain circuit that reaches its decision quickly and remains at this logic level until the comparator's clock input is a logic low.

Figure 4.8 also shows four transistors used to short out the cross coupled inverters. These transistors are turned on by clock inverter A when the clock input to this comparator is low, to force the comparator's positive and negative outputs low.

\subsubsection{Negative/Positive Tuning Capacitor Circuits}

In an ideal circuit, one without manufacturing defects, the current flowing through each transistor in the comparator's differential pair is entirely due to the gate voltage applied to each differential pair transistor. However, manufactured devices have variation. The result of this variation is that the comparator's transistors have different threshold voltages and also an undesirable offset current [16]. The comparator can be calibrated by 
adding a capacitance mismatch at the two output nodes. This capacitance cancels the offset due to manufacturing variations such as threshold voltage mismatches [17].

Figure 4.9 shows the schematic diagram of the tuning capacitors used to tune the comparator when the manufacturing defects require adding capacitance to the comparator's positive output.

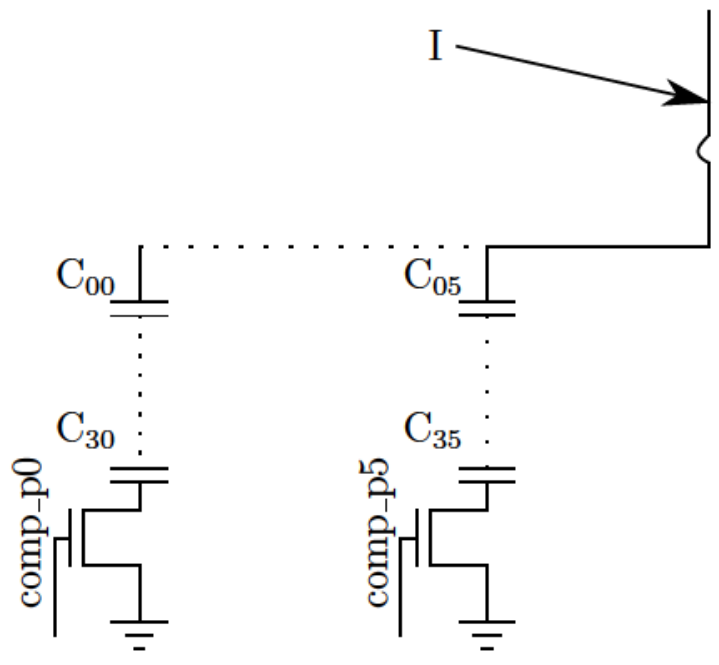

Figure 4.9: Comparator positive output tuning capacitors. Five sets of four series connected capacitors can be connected to the comparator's positive output node. These capacitors can be added to the comparator's positive output to compensate for an imbalance in capacitance between the differential pair and cross coupled inverters. Nominally, the capacitance of the positive and negative sides of the differential pair and cross coupled inverters are the same. If they differ due to manufacturing defects, the capacitors in this circuit can be added to compensate. Label I refers to labelled point I in Figure 4.4 .

Figure 4.10 shows the schematic diagram of the tuning capacitors used to tune the comparator when the manufacturing defects require adding capacitance to the comparator's negative output.

The calibration circuits shown in Figure 4.9 and Figure 4.10 allow capacitance to be added to the respective output node of the comparator. Each calibration circuit allows any combination of $299 \mathrm{fF}, 180 \mathrm{fF}, 121 \mathrm{fF}, 90.5 \mathrm{fF}, 75.9 \mathrm{fF}$, and $60.6 \mathrm{fF}$ to be added to the comparator's respective output node.

Monte Carlo analysis was performed with 200 points, process and random statistical variation. The parameters varied during each of the 200 the Monte Carlo runs was saved as a "corner" 2 that could be re-run as desired. This allowed the calibration of the comparator to be simulated at each of the 200 "corners" so that the optimal capacitance

\footnotetext{
${ }^{2}$ The use of the term "corner" is due how Monte Carlo simulation parameters are saved for use in re-simuations. It does not refer to either the commonly known process FF and SS "corners".
} 


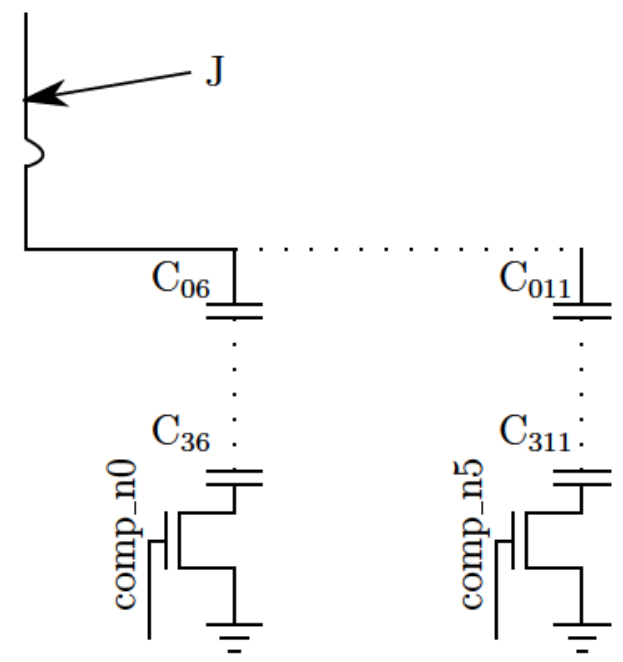

Figure 4.10: Comparator negative output tuning capacitors. Five sets of four series conected capacitors can be connected to the comparator's negative output node. These capacitors can be added to the comparator's negative output to compensate for a capacitor imbalance between the differential pair and cross coupled inverters. Nominally, the capacitance of the positive and negative sides of the differential pair and cross coupled inverters are the same. If they differ due to manufacturing defects, the capacitors in this circuit can be added to compensate. Label $\mathrm{J}$ refers to labelled point $\mathrm{J}$ in Figure 4.4 .

value required for calibration at each of these 200 corners could be determined. Analyzing the calibration of these 200 corners showed that adding capacitance in increments of $15 \mathrm{fF}$ compensated for manufacturing defects found while re-running the 200 Monte Carlo "corners".

A $15 \mathrm{fF}$ capacitance isn't large compared to wiring capacitance. Therefore, it was decided to use larger capacitors but to have a capacitance difference of $15 \mathrm{fF}$ between the negative and positive output nodes of the comparator. For example, adding $75.9 \mathrm{fF}$ to the positive node and $60.6 \mathrm{fF}$ to the negative node results in a difference of $15.3 \mathrm{fF}$. Depending on the amount of variation due to manufacturing and therefore, the amount of capacitance required to compensate determines which tuning capacitors to connect to the comparator's output nodes. Selecting which capacitors to connect is determined in a digital fashion by applying a logic 1 , or logic zero to each of the transistors shown in Figure 4.10 and Figure 4.9. Applying a logic 1 to any of the transistors, turns them on, and connects the associated capacitors to the respective comparator output node.

The capacitors used to implement the calibration circuits were vertical natural capacitors (VNCAP). This is a real capacitor with interdigitated metal fingers using the the same metal 1, IBM layer M1, metal layer. This capacitor was used instead of a MOS 
capacitor, a capacitor which has its capacitance based on the voltage applied to its gate, because if a MOS capacitor was used, the transient voltages applied to the comparator's output nodes will cause the MOS capacitor's capacitance to change over time. This changing voltage results in a varying capacitance, a capacitance that doesn't compensate for the non-varying manufacturing defect capacitance. The VNCAP is a fixed capacitance that doesn't vary as the transient voltage varies.

\subsubsection{Negative/Positive Output Buffer Circuits}

Figure 4.11 shows the schematic diagram of the output buffer for the negative output of the comparator. Figure 4.12 shows the schematic of the positive output buffer, which drives the positive output of the comparator. These buffers are required to isolate the differential pair and the cross coupled inverters from the loads they drive. This is necessary to ensure that the differential pair and the cross coupled inverters are not loaded by the circuits they drive. The differential pair and cross coupled inverters are very sensitive to the capacitance they drive, this is the reason for the calibration circuits described in the last section.

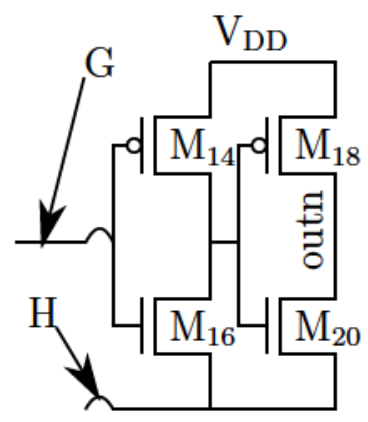

Figure 4.11: Comparator negative output buffer schematic. This buffer is composed of two inverters. The first inverter is made up of transistors $\mathrm{M}_{14}$ and $\mathrm{M}_{16}$. The second inverter is made up of transistors $\mathrm{M}_{18}$ and $\mathrm{M}_{20}$. Transistors $\mathrm{M}_{14}$ and $\mathrm{M}_{18}$ are each 640nm wide, 120nm long, and each have 1 finger. Transistors $\mathrm{M}_{16}$ and $\mathrm{M}_{20}$ are each $320 \mathrm{~nm}$ wide, $120 \mathrm{~nm}$ long, and each have 1 finger. Labels $\mathrm{G}$ and $\mathrm{H}$ refer to labelled points $\mathrm{G}$ and $\mathrm{H}$ in Figure 4.4 .

The sizes of the transistors in Figure 4.11 and Figure 4.12 were sized so the the differential pair and cross coupled inverters we not loaded by the level translating buffers they drive. 


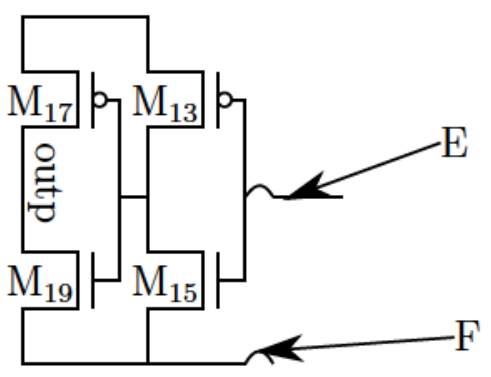

Figure 4.12: Comparator positive output buffer schematic. This buffer is composed of two inverters. The first inverter is made up of transistors $\mathrm{M}_{13}$ and $\mathrm{M}_{15}$. The second inverter is made up of transistors $\mathrm{M}_{17}$ and $\mathrm{M}_{19}$. Transistors $\mathrm{M}_{13}$ and $\mathrm{M}_{17}$ are each $640 \mathrm{~nm}$ wide, $120 \mathrm{~nm}$ long, and each have 1 finger. Transistors $\mathrm{M}_{15}$ and $\mathrm{M}_{19}$ are each $320 \mathrm{~nm}$ wide, $120 \mathrm{~nm}$ long, and each have 1 finger. Labels $\mathrm{E}$ and $\mathrm{F}$ refer to labelled points $\mathrm{E}$ and $\mathrm{F}$ in Figure 4.4 .

\subsubsection{Capacitor Array Circuit}

The charge sharing SAR $\mid$ ADC compares the voltage generated by its capacitor array to the voltage output by its $\mathrm{S} / \mathrm{H}$ to determine if the capacitor array's voltage approximates the sampled analog signal. The capacitor array's voltage is controlled by a SAR logic block. Each capacitor in the capacitor array is connected to a switch that is controlled by the SAR logic. The SAR logic uses the switches to set the voltage of each capacitor to either $\mathrm{V}_{\text {ref }}, 0 \mathrm{~V}$, or a voltage between $\mathrm{V}_{\text {ref }}$ and $0 \mathrm{~V}$.

Figure 4.13 shows the layout of the capacitor array used by this charge sharing SAR ADC.

Figure 4.13 implements 15 capacitors, each made up of two minimum sized two-layer MIM capacitors (known as a dualmimcap) from the IBM CMRF8SF library. Figure 4.14 shows a side view of the layers used by this capacitor. As Figure 4.14 shows, this capacitor is actually made up of two capacitors. The first capacitor is formed by the two metal layers HY and QY that are separated by an insulator. The second capacitor is made up of the QY and LY layers that are separated by an insulator. The dualmimcap was used because it has the largest capacitance per unit area of all the capacitors available in the IBM CMRF8SF library. The dualmimcap capacitor's capacitance is $4.10 \mathrm{fF} / \mu \mathrm{m}^{2}$ where the HY and QY layers overlap.

Figure 4.13 shows that each of the 15 capacitors in the capacitor array is made up of two capacitors (i.e. there are two capacitor $\mathrm{C}_{1}$ 's). This allows the use of common centroid layout techniques to be used when laying out the capacitor array. Common 


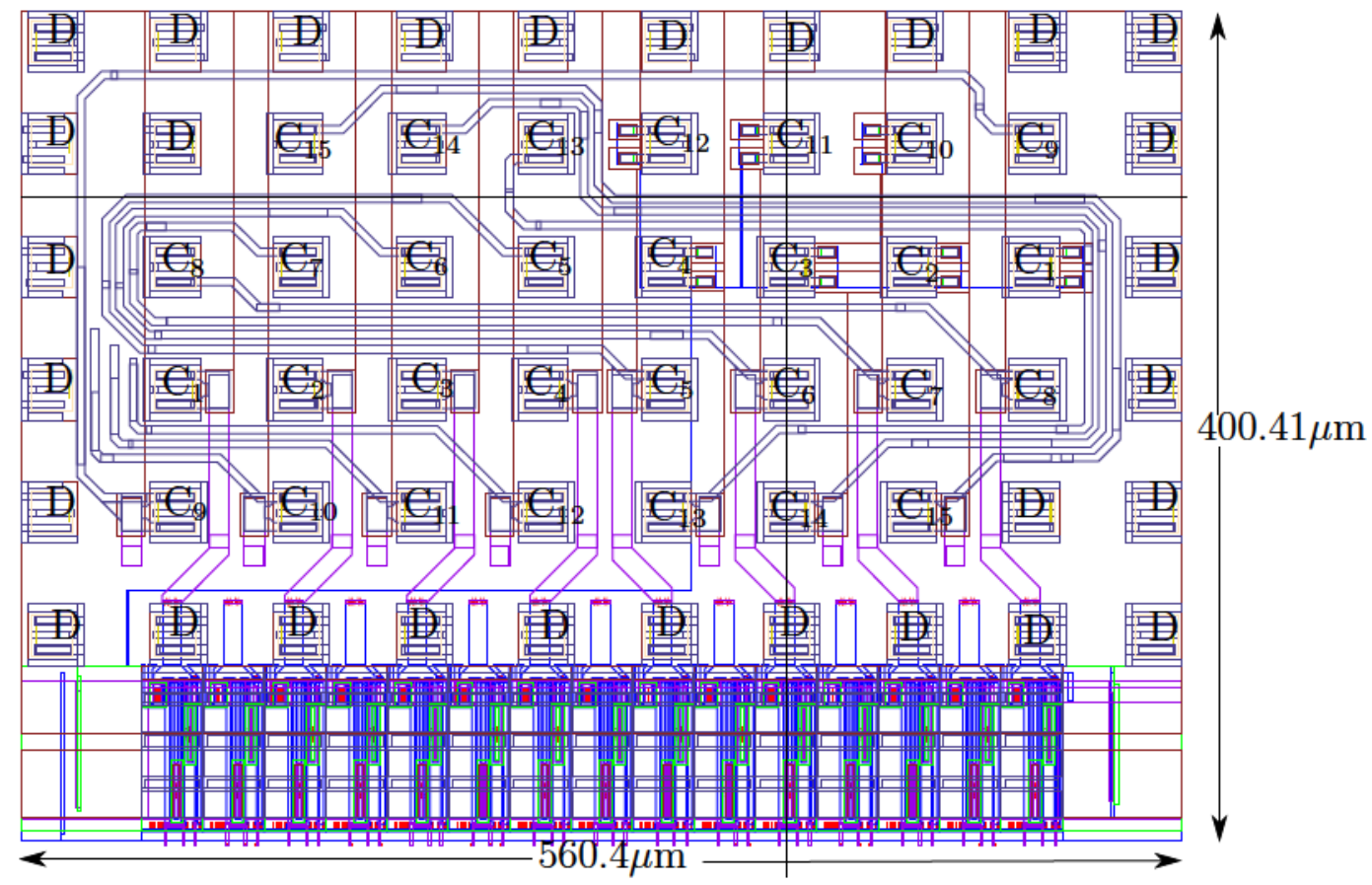

Figure 4.13: Capacitor array layout. " $\mathrm{D}$ " denotes a dummy capacitor and " $\mathrm{C}_{\mathrm{x}}$ " denotes a capacitor where $\mathrm{x}$ denotes the capacitor number (i.e. $\mathrm{C}_{4}$ denotes capacitor 4) between 1 and 15 . The capacitor array layout is $400.41 \mu \mathrm{m}$ tall and $560.4 \mu \mathrm{m}$ wide.

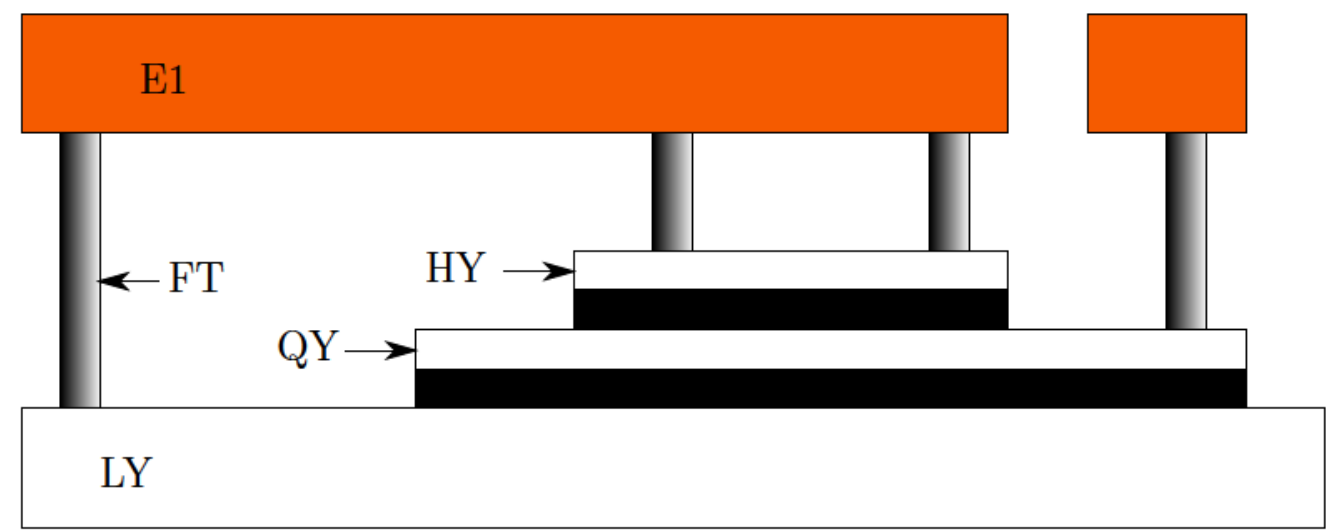

Figure 4.14: Cross-sectional view of the layer stackup used by the dualmimcap capacitor. The dualmimcap is a capacitor available in the IBM $0.13 \mu \mathrm{m}$ CMRF8SF library. The capacitor has a capacitance of $4.10 \mathrm{fF} / \mu \mathrm{m}^{2}$ in the region where the $\mathrm{HY}$ and QY plates overlap.

centroid layout techniques are used to reduce the effects of gradient variation such as oxide thickness that occur as a result of the manufacturing process. The commonly accepted basic assumption is that these gradients vary linearly over small distances, so, the effect of a mismatch due to these gradients can be reduced by reducing the separation of the centroids of the laid out devices. This is true because the magnitude of the mismatch equals the product of the distance between the centroids and the magnitude of the gradient along the axis of separation [18]. Figure 4.15 shows how the capacitor 
array was laid out to ensure the centroid of this layout was in the center of the capacitor array. As Figure 4.15 shows, the two rows of capacitors below the dashed line labelled as "Horizontal axis of symmetry" were flipped horizontally above this line (shown as an arrow with a solid line in Figure 4.15) and then flipped again, vertically about the dotted line labelled as "Vertical axis of symmetry" (shown as an arrow with a dotted line in Figure 4.15). Flipping these capacitors in this manner caused the two rows of capacitors above the "Horizontal axis of symmetry" to be the mirror image, both horizontally and vertically, of the two rows of capacitors below the "Horizontal axis of symmetry", resulting in the centroid of this layout to be at the center of the capacitors.

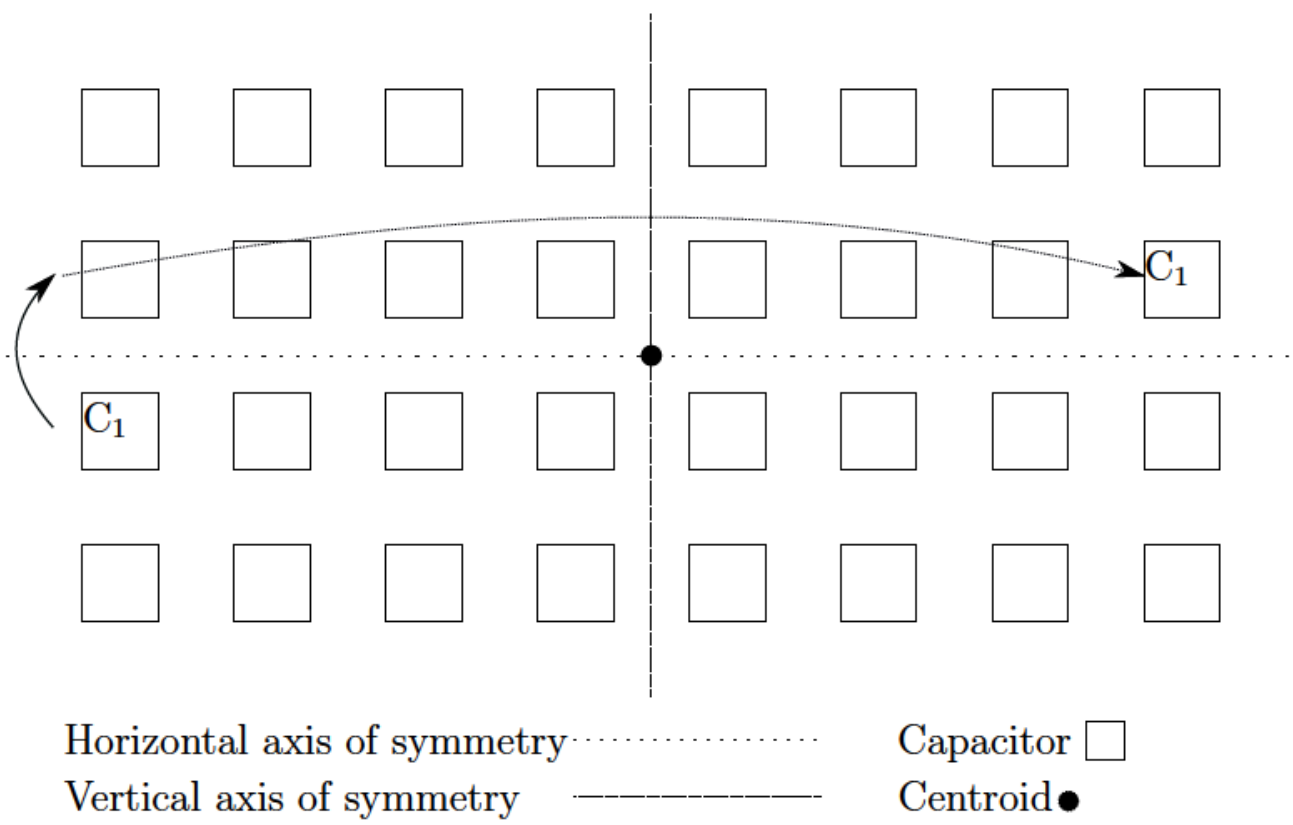

Figure 4.15: 15 capacitor common centroid diagram. The two rows of capacitors below the line denoted as "Horizontal axis of symmetry" were flipped, horizontally, above this line. The arrow with a solid line shows this flip. These horizontally flipped capacitors are then flipped vertically about the line denoted "Vertical axis of symmetry". This vertical flip is shown by the arrow with the dotted line. The centroid of this capacitor array layout is therefore at the center of the layout to compensate for linear gradients that occur as a result of the manufacturing process. 
Figure 4.13 also shows capacitors labelled "D", for dummy. These dummy capacitors were added into the layout of the capacitor array to improve matching of capacitors $\mathrm{C}_{1}$ through $\mathrm{C}_{15}$. A major issue that effects capacitor matching is the amount of etching the capacitors are subjected to. The size of an area etched determines the "etch rate". This means that a larger area to be etched will have a higher etch rate. Dummy capacitors are added to the layout to ensure the etching of all capacitors in the capacitor array are exposed to the same etch rate, and therefore, the same amount on undercut. Undercut occurs during the manufacturing process and is a result of the excessive removal of material under the current layer of material being processed. Figure 4.16 shows how adding dummy capacitors effect etch rate and Figure 4.17 shows how undercut is effected by etch rate, and therefore, the capacitance value of the capacitors in the array after manufacturing.

An example of how the capacitors would be processed differently is described in [10]. [10] describes matching resistors, but this same issues and methods to resolve them are true for capacitors. Dummy elements are used to improve matching between two or more elements [10]. 


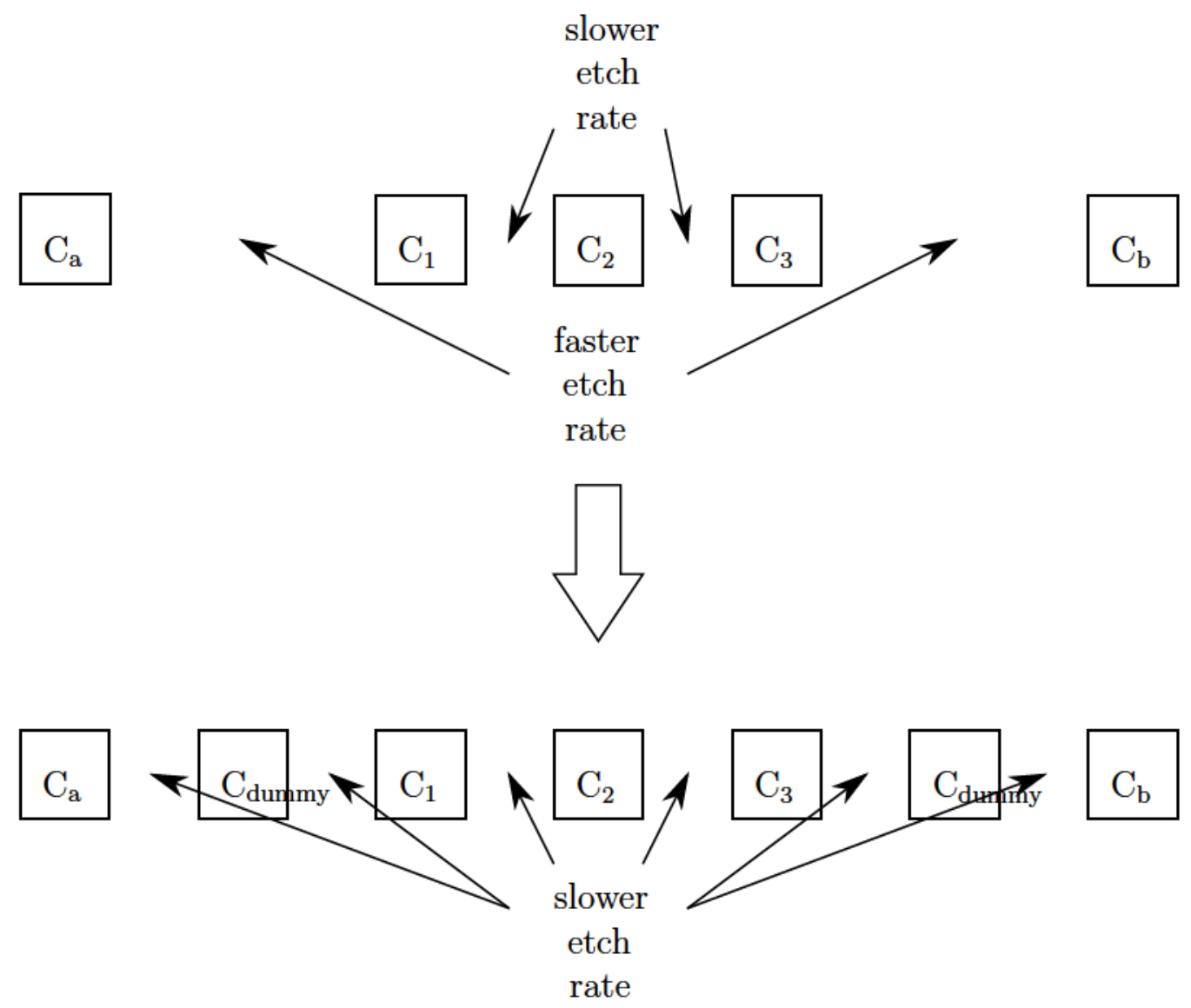

Figure 4.16: Example of how dummy capacitors affect etch rate. The top row of capacitors are spaced so that the amount of etch between capacitors $\mathrm{C}_{\mathrm{a}}, \mathrm{C}_{1}$ and $\mathrm{C}_{3}, \mathrm{C}_{\mathrm{b}}$ is greater than between $\mathrm{C}_{1}$ and $\mathrm{C}_{2}$ and $\mathrm{C}_{3}$. Therefore, the capacitance of capacitor $\mathrm{C}_{1}$ is not the same as capacitor $\mathrm{C}_{2}$ and the capacitance of capacitor $\mathrm{C}_{2}$ isn't the same as capacitor $\mathrm{C}_{3}$. Adding dummy capacitors in the second row of capacitors spaces them so that the amount of etch each capacitor is exposed to is the same. With the same etch rate, all capacitors in the second row have the same capacitance.
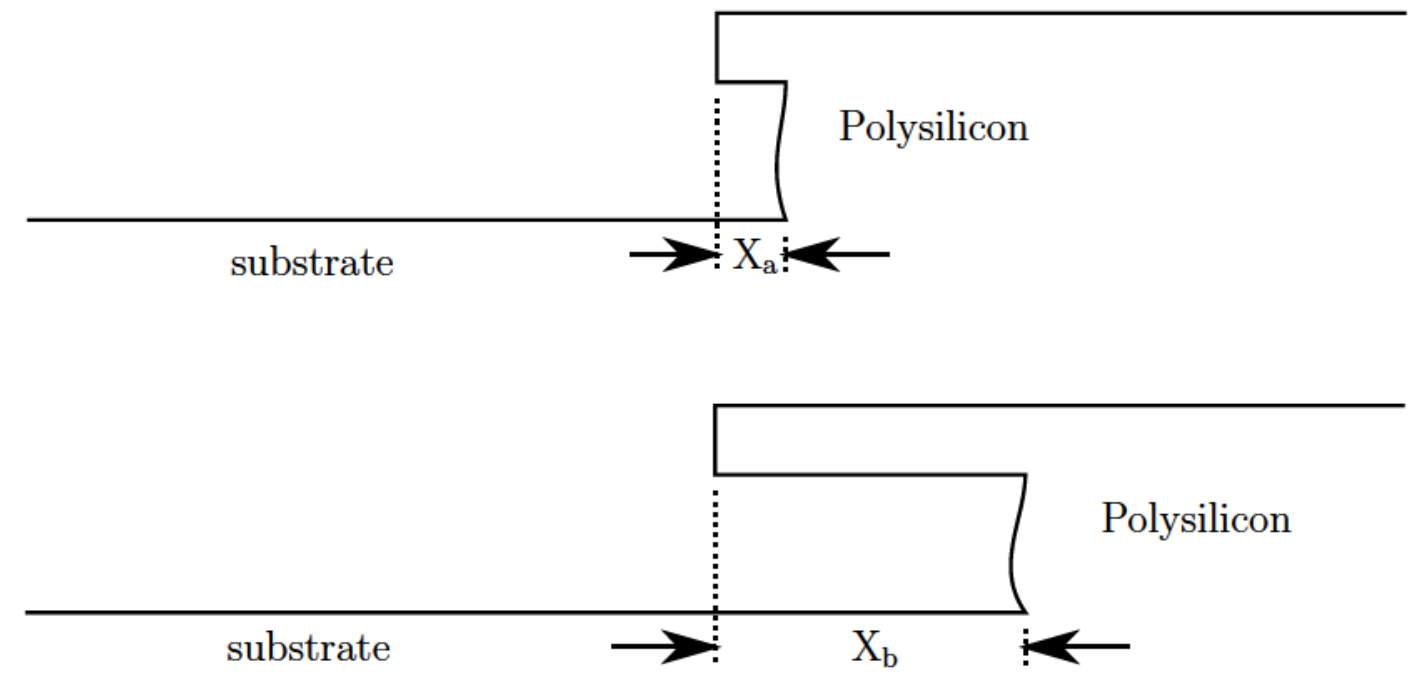

Figure 4.17: Example of how the etch rate effects the amount of undercut. The higher the etch rate the higher the undercut. $\mathrm{X}_{\mathrm{a}}$ is the amount of undercut when there is less etch rate. $\mathrm{X}_{\mathrm{b}}$ is the amount of undercut when there is more etch rate. 


\subsubsection{SAR Digital Logic Circuit}

The SAR digital logic block in the charge sharing SAR ADC controls the capacitor array to generate a voltage that the ADC compares to a sampled analog voltage in an attempt to convert the sampled analog voltage into an equivalent digital value. The layout of the SAR digital logic block used in the charge sharing SAR ADC is shown in Figure 4.18 .

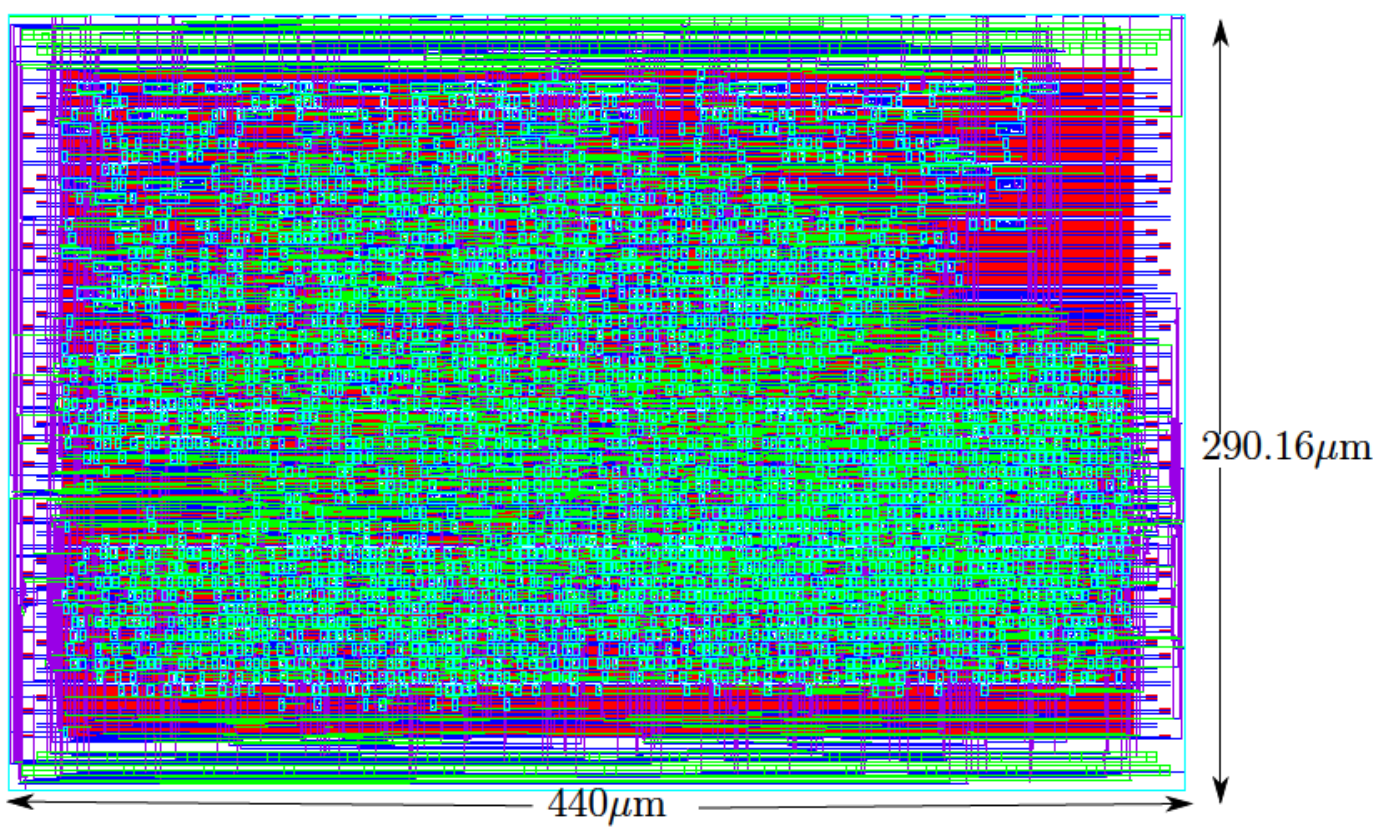

Figure 4.18: SAR layout showing placement of the standard cells (small green boxes), routes (horizontal and vertical wires), and filler cells (red fill). The SAR layout is $290.16 \mu \mathrm{m}$ tall and is $440 \mu \mathrm{m}$ wide.

The SAR logic block shown in Figure 4.18 implements an algorithm using standard cells specifically created for this thesis. The Canadian Microelectronics Corporation (CMC) can provide an ARM standard cell library for use with the IBM CMRF8SF $0.13 \mu \mathrm{m}$ library. Unfortunately, the ARM standard cell library does not include GDSII views. This means that the standard cells are represented by black boxes and can not be simulated in the Virtuoso environment. It was desirable to simulate the full charge sharing SAR ADC, including the SAR digital logic block in Virtuoso. So a custom standard cell library was developed. A second reason to develop a custom standard cell library was to take advantage of standardized PNR flows to synthesize, place, and route the SAR,

This custom standard cell library has a clock inverter (CLKINVX1), inverter (INVX1), two input NAND (NAND2X1), two input NOR (NOR2X1), asynchronously resetable 
flip-flop (DFFRHQX1), and an asynchronously setable flip-flop (DFFSHQX1). These cells are well defined and the interested reader can refer to [19], [20], and [21] for more information about how to design these cells.

\subsubsection{CLKINVX1}

CLKINVX1 is a clock inverter.

Clock trees are either built out of buffers or inverters. The choice of which cell to use to build the clock tree is based on which cell's output signal has the most symmetric rise and fall time. Standard inverters and buffers tend to either have output's with faster rise time than fall time or faster fall time than rise time. The problem with asymmetric rise and fall times is that this leads to what is known as duty cycle distortion. Duty cycle distortion is a form of clock jitter where the time the inverter or buffer's output takes to transition from a logic low to a logic high is different than the time its output transitions from a logic high to a logic low. The reason for creating a specific cell for the clock tree is to ensure that the cell has an output with symmetric rise and fall times. This was achieved by sizing the pfet and nfet transistors correctly. It was found that sizing the pfet to be 1.5 times the size of the nfet gave the most symmetric rise and fall times when driving four inverters (INVX1 cells from this library) with drive strength of 1 . The pfet's length is $420 \mathrm{~nm}$ and width is $120 \mathrm{~nm}$. The nfet's length is $280 \mathrm{~nm}$ and width is $120 \mathrm{~nm}$. The layout of the CLKINVX1 is shown in Figure 4.19 .

To ease placement, all the cells in a standard cell library are physically the same height. Standard cells are either 9 tracks or 12 tracks tall. Their width must be a multiple of the width of the smallest cell in the library, which in this library is a filler cell (the filler cell in this library is the FILL1). A 9 track tall cell has a height that is 9 times the minimum metal 1 wiring pitch. The metal 1 wiring pitch is the minimum width of metal 1 plus the minimum metal 1 spacing. A 12 track cell is the height that is 12 times the minimum metal 1 wiring pitch. The IBM CMRF8SF $0.13 \mu \mathrm{m}$ library's metal 1 minimum width is $0.16 \mu \mathrm{m}$ and the minimum metal 1 spacing is also $0.16 \mu \mathrm{m}$, therefore, the height of the CLKINVX1 is $3.84 \mu \mathrm{m}$. The cells in this custom standard cell 


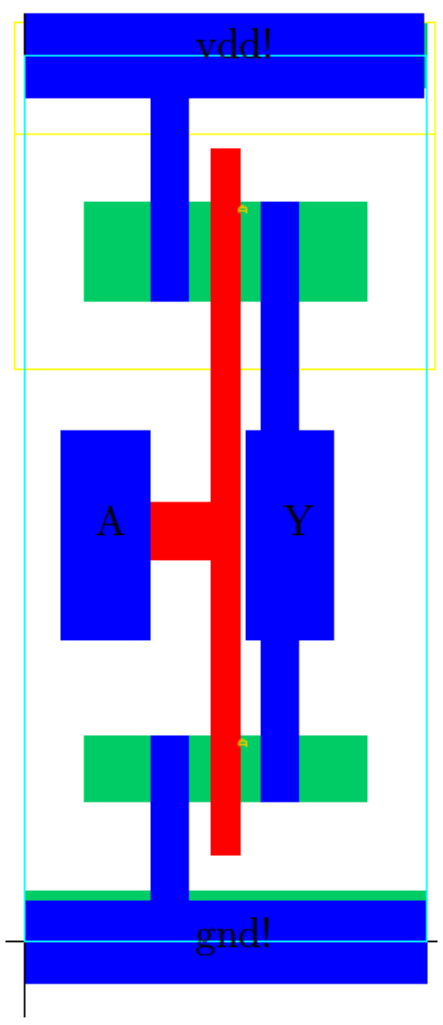

Figure 4.19: CLKINVX1 layout showing the nfet and the pfet transistors and their connection. The input pin is labelled "A", the output is labelled "Y", the nwell connection is labelled "vdd!" and the subtrate connection is labelled "gnd!". The CLKINVX1 standard cell is $3.84 \mu \mathrm{m}$ tall and is $1.74 \mu \mathrm{m}$ wide.

library are 12 tracks tall. Using 12 track cells allowed the standard cells to be routed only using poly and metal 1 . Nine track tall cells would require higher layers of metal in the standard cells, which would remove layers for signal routing. It was also found that 9 track cells were much longer than 12 track cells in the CMRF8SF library due to design rule check (DRC) spacing rules.

\subsubsection{INVX1}

The INVX1 is an inverter. This INVX1's pfet and nfet transistors were sized so that the INVX1 could drive four other INVX1's spaced by approximately $1 \mu \mathrm{m}$ vertically and $1 \mu \mathrm{m}$ horizontally, without a logic high degrading below $10 \%$. The pfet width is $380 \mathrm{~nm}$ and length is $120 \mathrm{~nm}$. The nfet is also $380 \mathrm{~nm}$ wide and $120 \mathrm{~nm}$ long. The layout of the INVX1 is shown in Figure 4.20. 


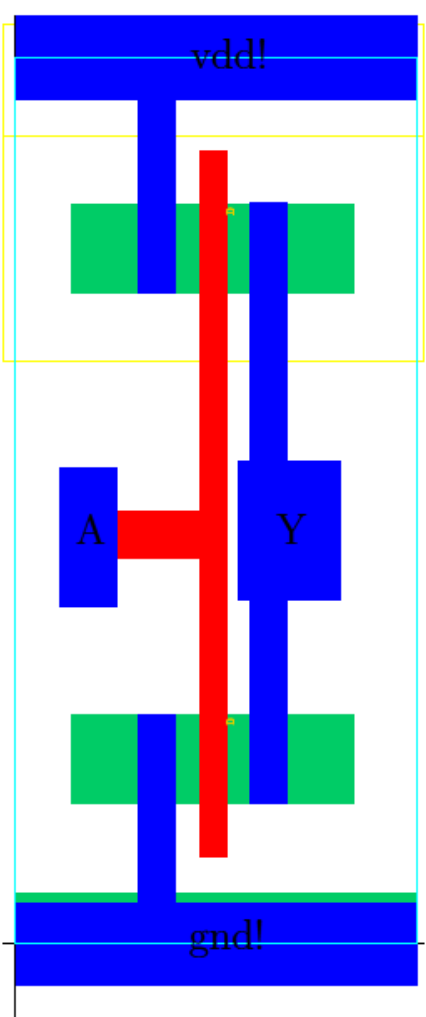

Figure 4.20: INVX1 layout showing the nfet and the pfet transistors and their connection. The input pin is labelled "A", the output is labelled "Y", the nwell connection is labelled "vdd!" and the subtrate connection is labelled "gnd!". The INVX1 standard cell is $3.84 \mu \mathrm{m}$ tall and is $1.74 \mu \mathrm{m}$ wide.

\subsubsection{NAND2X1}

The NAND2X1 is a two input NAND gate. This NAND2X1's pfet and nfet transistors were sized with the same width and length as the INVX1. The layout of the NAND2X1 is shown in Figure 4.21 .

\subsubsection{NOR2X1}

NOR2X1 is a two input NOR gate. The NOR2X1's pfet and nfet transistors have the same width and length as the INVX1's pfet and nfet transistors. The layout of the NOR2X1 is shown in Figure 4.22 . 


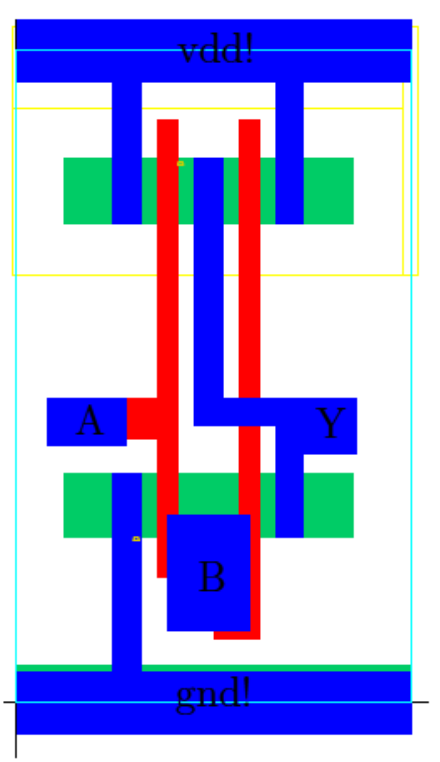

Figure 4.21: NAND2X1 layout showing the two nfet and two pfet transistors and their connection. The input pins are labelled "A" and "B", the output is labelled "Y", the nwell connection is labelled "vdd!" and the subtrate connection is labelled "gnd!". The NAND2X1 standard cell is $3.84 \mu \mathrm{m}$ tall and is $2.32 \mu \mathrm{m}$ wide.

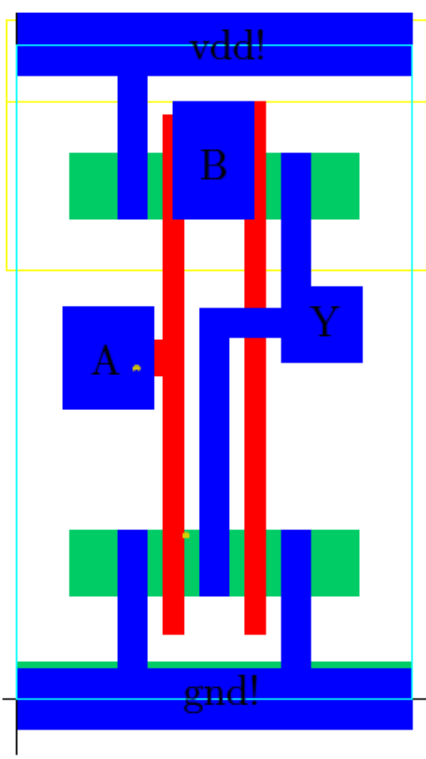

Figure 4.22: NOR2X1 layout showing the two nfet and two pfet transistors and their connection. The input pins are labelled "A" and "B", the output is labelled "Y", the nwell connection is labelled "vdd!" and the subtrate connection is labelled "gnd!". The NOR2X1 standard cell is $3.84 \mu \mathrm{m}$ tall and is $2.32 \mu \mathrm{m}$ wide.

\subsubsection{DFFRHQX1}

The DFFRHQX1 is a D flip-flop with asynchronous reset. The schematic diagram of the DFFRHQX1 is shown in Figure 4.23. The sizes of the transistors for Figure 4.23 are shown in Table 4.1. The layout of the DFFRHQX1 is shown in Figure 4.24. 


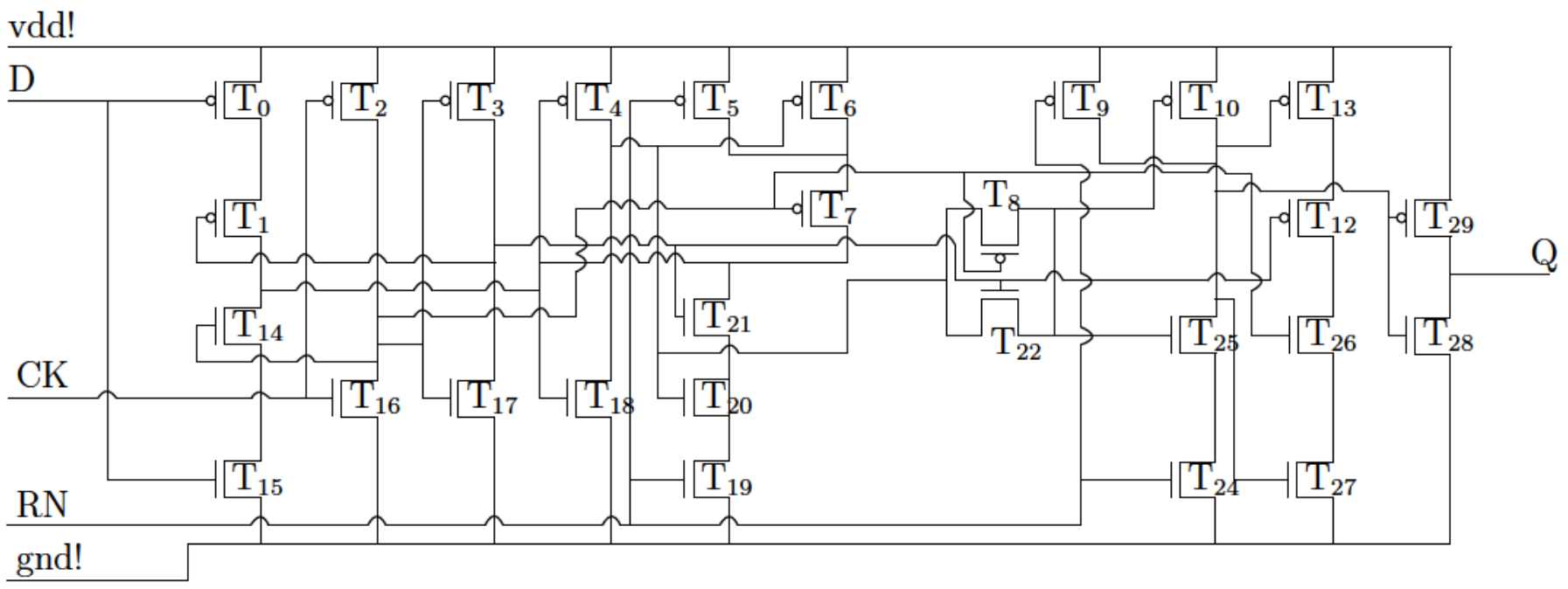

Figure 4.23: DFFRHQX1 transistor level schematic. The sizes of transistors in this diagram are provided in Table 4.1 .

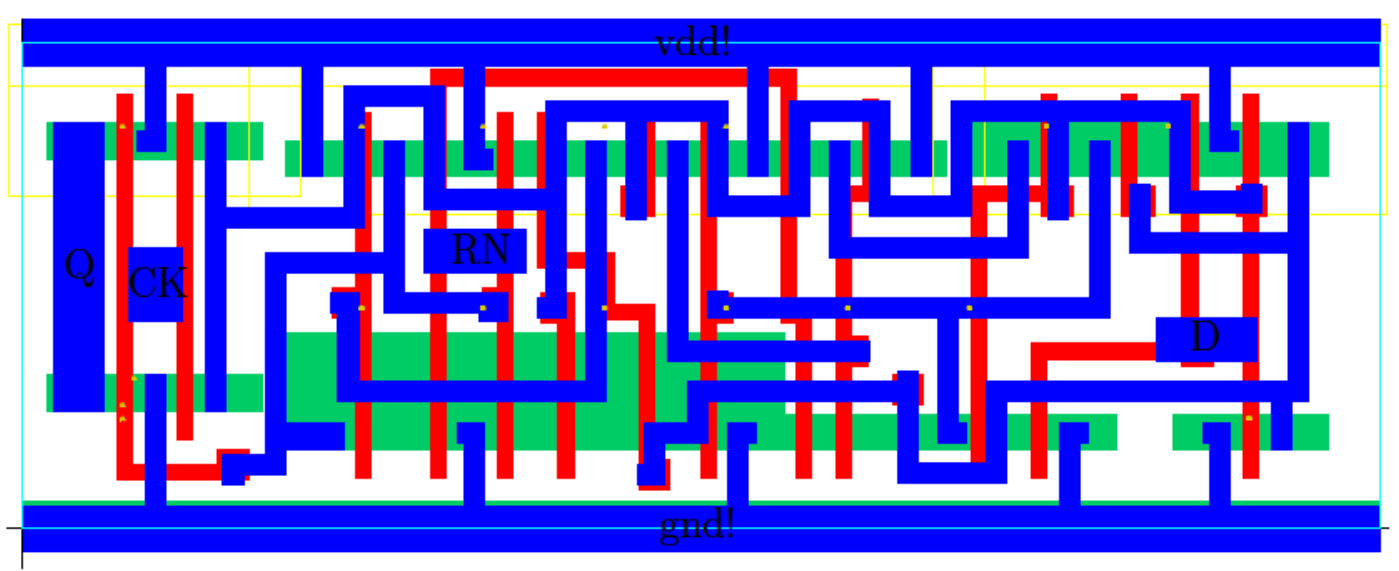

Figure 4.24: DFFRHQX1 layout showing 14 nfet and 14 pfet transistors and their connection. The input pin is labelled " $\mathrm{D}$ ", the output is labelled "Q", the asynchronous reset input pint is labelled "RN", the clock input pin is labelled "CK", the nwell connection is labelled "vdd!" and the subtrate connection is labelled "gnd!". The DFFRHQX1 standard cell is $3.84 \mu \mathrm{m}$ tall and is $10.73 \mu \mathrm{m}$ wide. 


\begin{tabular}{|c|c|c|c|}
\hline Transistor Name & Width & Length & Number of Fingers \\
\hline $\mathrm{T}_{0}$ & $280 \mathrm{~nm}$ & $120 \mathrm{~nm}$ & 1 \\
\hline $\mathrm{T}_{1}$ & $280 \mathrm{~nm}$ & $120 \mathrm{~nm}$ & 1 \\
\hline $\mathrm{T}_{2}$ & $280 \mathrm{~nm}$ & $120 \mathrm{~nm}$ & 1 \\
\hline $\mathrm{T}_{3}$ & $280 \mathrm{~nm}$ & $120 \mathrm{~nm}$ & 1 \\
\hline $\mathrm{T}_{4}$ & $280 \mathrm{~nm}$ & $120 \mathrm{~nm}$ & 1 \\
\hline $\mathrm{T}_{5}$ & $280 \mathrm{~nm}$ & $120 \mathrm{~nm}$ & 1 \\
\hline $\mathrm{T}_{6}$ & $280 \mathrm{~nm}$ & $120 \mathrm{~nm}$ & 1 \\
\hline $\mathrm{T}_{7}$ & $280 \mathrm{~nm}$ & $120 \mathrm{~nm}$ & 1 \\
\hline $\mathrm{T}_{8}$ & $280 \mathrm{~nm}$ & $120 \mathrm{~nm}$ & 1 \\
\hline $\mathrm{T}_{9}$ & $280 \mathrm{~nm}$ & $120 \mathrm{~nm}$ & 1 \\
\hline $\mathrm{T}_{10}$ & $280 \mathrm{~nm}$ & $120 \mathrm{~nm}$ & 1 \\
\hline $\mathrm{T}_{11}$ & $280 \mathrm{~nm}$ & $120 \mathrm{~nm}$ & 1 \\
\hline $\mathrm{T}_{24}$ & $280 \mathrm{~nm}$ & $120 \mathrm{~nm}$ & 1 \\
\hline $\mathrm{T}_{26}$ & $380 \mathrm{~nm}$ & $120 \mathrm{~nm}$ & 1 \\
\hline $\mathrm{T}_{27}$ & $280 \mathrm{~nm}$ & $120 \mathrm{~nm}$ & 1 \\
\hline $\mathrm{T}_{28}$ & $380 \mathrm{~nm}$ & $120 \mathrm{~nm}$ & 1 \\
\hline $\mathrm{T}_{29}$ & $920 \mathrm{~nm}$ & $120 \mathrm{~nm}$ & 1 \\
\hline $\mathrm{T}_{31}$ & $320 \mathrm{~nm}$ & $120 \mathrm{~nm}$ & 1 \\
\hline $\mathrm{T}_{32}$ & $320 \mathrm{~nm}$ & $120 \mathrm{~nm}$ & 1 \\
\hline $\mathrm{T}_{33}$ & $320 \mathrm{~nm}$ & $120 \mathrm{~nm}$ & 1 \\
\hline $\mathrm{T}_{34}$ & $920 \mathrm{~nm}$ & $120 \mathrm{~nm}$ & 1 \\
\hline $\mathrm{T}_{35}$ & $920 \mathrm{~nm}$ & $120 \mathrm{~nm}$ & 1 \\
\hline $\mathrm{T}_{36}$ & $920 \mathrm{~nm}$ & $120 \mathrm{~nm}$ & 1 \\
\hline $\mathrm{T}_{37}$ & $920 \mathrm{~nm}$ & $120 \mathrm{~nm}$ & 1 \\
\hline $\mathrm{T}_{38}$ & $920 \mathrm{~nm}$ & $120 \mathrm{~nm}$ & 1 \\
\hline $\mathrm{T}_{39}$ & $920 \mathrm{~nm}$ & $120 \mathrm{~nm}$ & 1 \\
\hline $\mathrm{T}_{40}$ & $280 \mathrm{~nm}$ & $120 \mathrm{~nm}$ & 1 \\
\hline
\end{tabular}

Table 4.1: Figure 4.23 DFFRHQX1 transistor sizes. 


\subsubsection{DFFSHQX1}

The DFFSHQX1 is a D flip-flop with asynchronous set. The schematic diagram of the DFFSHQX1 is shown in Figure 4.25. The sizes of the transistors for Figure 4.25 are shown in Table 4.2. The layout of the DFFSHQX1 is shown in Figure 4.26.

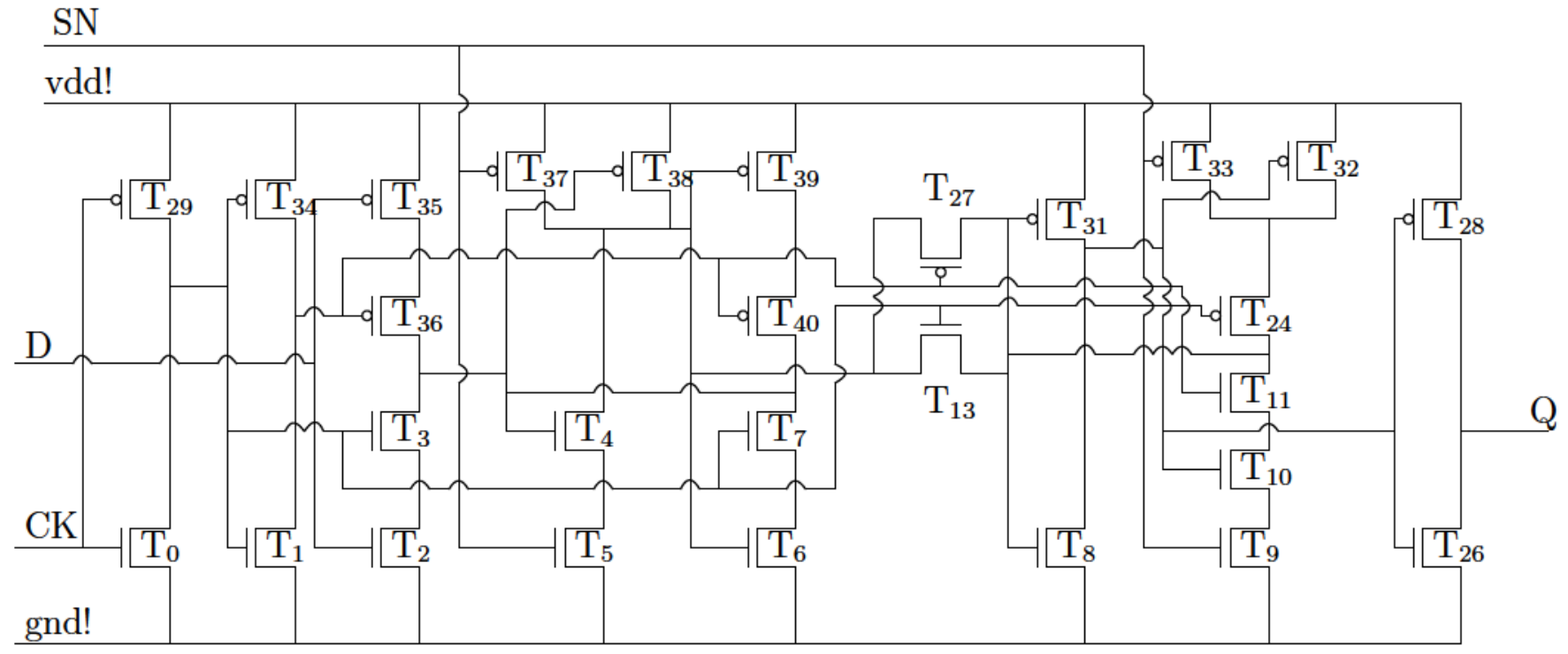

Figure 4.25: DFFSHQX1 transistor level schematic. The sizes of transistors in this diagram are provided in Table 4.2 .

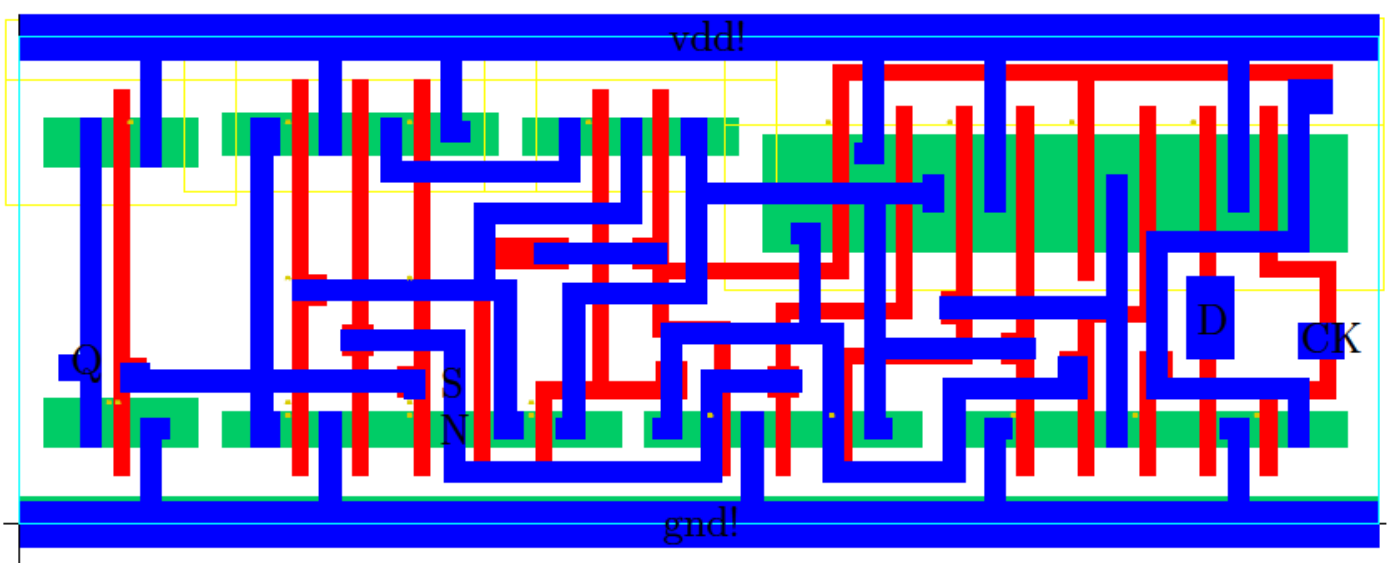

Figure 4.26: DFFSHQX1 layout showing 14 nfet and 14 pfet transistors and their connection. The input pin is labelled "D", the output is labelled "Q", the asynchronous set input pint is labelled "SN", the clock input pin is labelled "CK", the nwell connection is labelled "vdd!" and the subtrate connection is labelled "gnd!". The DFFSHQX1 standard cell is $3.84 \mu \mathrm{m}$ tall and is $10.73 \mu \mathrm{m}$ wide. 


\begin{tabular}{|c|c|c|c|}
\hline Transistor Name & Width & Length & Number of Fingers \\
\hline $\mathrm{T}_{0}$ & $420 \mathrm{~nm}$ & $120 \mathrm{~nm}$ & 1 \\
\hline $\mathrm{T}_{1}$ & $420 \mathrm{~nm}$ & $120 \mathrm{~nm}$ & 1 \\
\hline $\mathrm{T}_{2}$ & $280 \mathrm{~nm}$ & $120 \mathrm{~nm}$ & 1 \\
\hline $\mathrm{T}_{3}$ & $420 \mathrm{~nm}$ & $120 \mathrm{~nm}$ & 1 \\
\hline $\mathrm{T}_{4}$ & $280 \mathrm{~nm}$ & $120 \mathrm{~nm}$ & 1 \\
\hline $\mathrm{T}_{5}$ & $280 \mathrm{~nm}$ & $120 \mathrm{~nm}$ & 1 \\
\hline $\mathrm{T}_{6}$ & $280 \mathrm{~nm}$ & $120 \mathrm{~nm}$ & 1 \\
\hline $\mathrm{T}_{7}$ & $420 \mathrm{~nm}$ & $120 \mathrm{~nm}$ & 1 \\
\hline $\mathrm{T}_{8}$ & $280 \mathrm{~nm}$ & $120 \mathrm{~nm}$ & 1 \\
\hline $\mathrm{T}_{9}$ & $280 \mathrm{~nm}$ & $120 \mathrm{~nm}$ & 1 \\
\hline $\mathrm{T}_{10}$ & $280 \mathrm{~nm}$ & $120 \mathrm{~nm}$ & 1 \\
\hline $\mathrm{T}_{12}$ & $280 \mathrm{~nm}$ & $120 \mathrm{~nm}$ & 1 \\
\hline $\mathrm{T}_{13}$ & $280 \mathrm{~nm}$ & $120 \mathrm{~nm}$ & 1 \\
\hline $\mathrm{T}_{14}$ & $280 \mathrm{~nm}$ & $120 \mathrm{~nm}$ & 1 \\
\hline $\mathrm{T}_{15}$ & $280 \mathrm{~nm}$ & $120 \mathrm{~nm}$ & 1 \\
\hline $\mathrm{T}_{16}$ & $280 \mathrm{~nm}$ & $120 \mathrm{~nm}$ & 1 \\
\hline $\mathrm{T}_{17}$ & $280 \mathrm{~nm}$ & $120 \mathrm{~nm}$ & 1 \\
\hline $\mathrm{T}_{18}$ & $920 \mathrm{~nm}$ & $120 \mathrm{~nm}$ & 1 \\
\hline $\mathrm{T}_{19}$ & $280 \mathrm{~nm}$ & $120 \mathrm{~nm}$ & 1 \\
\hline $\mathrm{T}_{20}$ & $280 \mathrm{~nm}$ & $120 \mathrm{~nm}$ & 1 \\
\hline $\mathrm{T}_{21}$ & $280 \mathrm{~nm}$ & $120 \mathrm{~nm}$ & 1 \\
\hline $\mathrm{T}_{22}$ & $920 \mathrm{~nm}$ & $120 \mathrm{~nm}$ & 1 \\
\hline $\mathrm{T}_{24}$ & $920 \mathrm{~nm}$ & $120 \mathrm{~nm}$ & 1 \\
\hline $\mathrm{T}_{25}$ & $920 \mathrm{~nm}$ & $120 \mathrm{~nm}$ & 1 \\
\hline $\mathrm{T}_{26}$ & $920 \mathrm{~nm}$ & $120 \mathrm{~nm}$ & 1 \\
\hline $\mathrm{T}_{27}$ & $920 \mathrm{~nm}$ & $120 \mathrm{~nm}$ & 1 \\
\hline $\mathrm{T}_{28}$ & $280 \mathrm{~nm}$ & $120 \mathrm{~nm}$ & 1 \\
\hline
\end{tabular}

Table 4.2: Figure 4.25 DFFSHQX1 transistor sizes. 


\subsubsection{FILL1}

Like all technology libraries, CMRF8SF has a DRC rule specifying the minimum spacing between nwells. In order to pack the standard cells in a row as tightly as possible, and mitigate the nwell spacing rule, all standard cells in a row share the same nwell. This results in an nwell that extends the entire length of a row. The width of standard cells are a multiple of the filler cell, FILL1's width. This means the the length of a row is also a multiple of the FILL1's width and all CLKINVX1, INVX1, NOR2X1, NAND2X1, DFFRHQX1, DFFSHQX1 cells that aren't abutted horizontally, have one or more FILL1 cells placed between them.

The FILL1 cell's layout is shown in Figure 4.27.

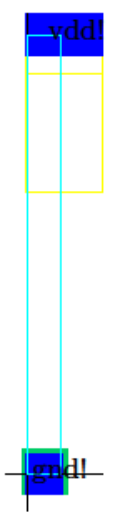

Figure 4.27: FILL1 layout. This cell has no input pins nor output pins. The nwell connection is labelled "vdd!" and the subtrate connection is labelled "gnd!". The FILL1 standard cell is $3.84 \mu \mathrm{m}$ tall and is $0.29 \mu \mathrm{m}$ wide.

Standardized PNR flows give designers a flexible set of industry standard tools to generate a synthesized, placed, and routed design that has no timing violations and no DRC violations in a short period of time. The advantage of the PNR flow is that when a bug is found in the design, the RTL can be modified and a new synthesized, placed, and routed design can be generated quickly. However, the main advantage of the PNR flow is that it allows designers to focus on fixing bugs instead of being forced to worry about the logic's implementation.

Figure 4.28 shows the standard $\mathrm{PNR}$ flow. The inputs to the flow are a Verilog RTL code, the timing and placement information of the standard cell library, and timing constraints. Synopsys Deign Compiler (DC) was used to convert the Verilog RTL into a 
structural RTL file. Structural RTL contains only the instantiated standard cells, their interconnecting nets, and input and output ports. This structural RTL is then imported into Cadence Encounter to create the floorplan. The floorplan defines the placement of the input and output pins, the power grid, and rows to place the standard cells in. The design exchange format (DEF) file, that represents the floorplan, is exported from Encounter and used as a constraint by [DC during subsequent synthesis runs. Without a DEF file, DC synthesizes logic using what are known as "wire load models". Wire load models are statistical estimated capacitive loads applied to the inputs and output of standard cells. These models allow DC to estimate the timing of the standard cells and this timing is the basis for how DC selects not only which logic cells to use. The physical information in the DEF file allows physically aware synthesis tools like $\mathrm{DC} \mathrm{S}^{3}$ to more accurately synthesize the Verilog RTL. The physically aware synthesis uses a more accurate estimate of the interconnection between logic cells called a "Steiner Route" which is a form of "Manhattan" routing. This routing is extracted to estimate the capacitance connected to the standard cell's inputs and output. A standard cell's timing is based on the capacitance it drives and the transition time of the cell's inputs. The information in the $\mathrm{DEF}$ file also allows $\mathrm{DC}$ to perform a simple form of placement so that buffers can be inserted into logic paths (paths using standard cells between flip-flops). The result is that synthesis based on a $\mathrm{DEF}$ converges and closes timing more quickly than a design synthesized using wire load models because the logic chosen during synthesis requires less optimization during placement. Physical synthesis has replaced synthesis based on wire load models because it is well known that logic optimization during the placement phase may not converge so it is a good design practice to optimize logic at the synthesis stage. The placement tool is not as good at logic optimization as a synthesis tool.

The design is closed when the design is timing clean, has no DRC violations, and all

\footnotetext{
${ }^{3}$ Design Compiler is commonly used as the term used to describe the more broad set of tools such as DC, Design Compiler Topological (DC-T), and Design Compiler Graphical (DC-G). It is common to simply refer to all of these tools as DC because many PNR flows launch DC and then use the different modes (Topological and Graphical) on the fly by invoking the required modes as needed. For example, the PNR script can be written to always launch $\mathrm{DC}$ and then invoke the topological physical synthesis options if a DEF file is provided or to invoke the graphical physical synthesis options if the library contains foundry yield information.
} 


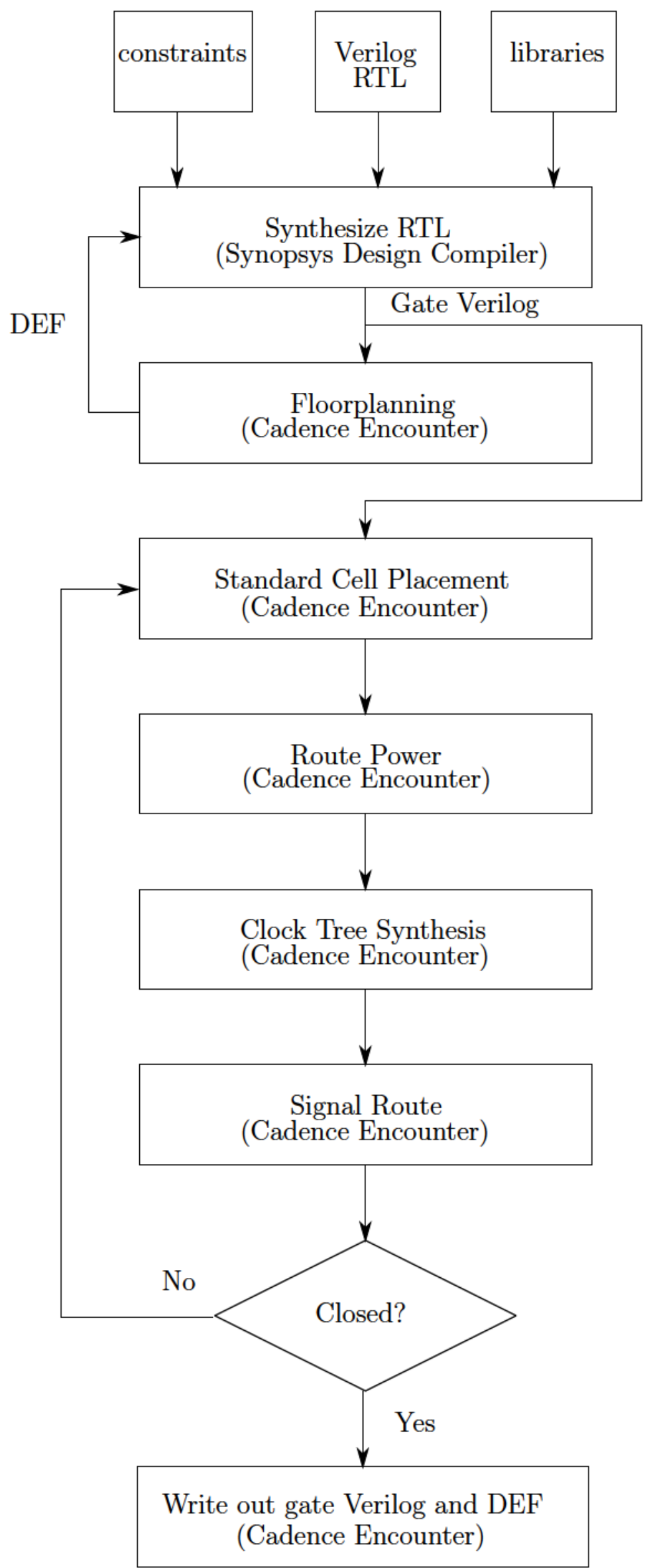

Figure 4.28: $\mathrm{PNR}$ Flow. 
of the wires are routed without shorts. The design iterates through this flow until all of these conditions are satisfied.

\subsubsection{Verilog RTL}

Writing Verilog RTL code allows designers to use abstraction to create very complex logic that would not be possible if the designer were to write a more structural RTL. Abstraction moves the designer away from the concerns of which specific cells to instantiate and how to connect them and allows them to focus on implementing complex algorithms. Verilog RTL designs rely on standard synthesis and PNR tools to take care of implementing specific gates, their drive strengths, and their connectivity.

The Verilog RTL code implements a finite state machine (FSM). A FSM uses logic gates (i.e. NAND, NOR, AND, OR, INV, XOR, XNOR gates) and flip-flops to implement an algorithm to control the capacitor array's output voltage. The FSM is broken up into a finite number of states that uses a clock to trigger the transition from one state to the next. Two common FSM are the Mealy and Moore state machines. A Mealy type FSM s outputs are dependent on the current state and one or more of the input signals.

A Moore type FSM s output is only due to the current state.

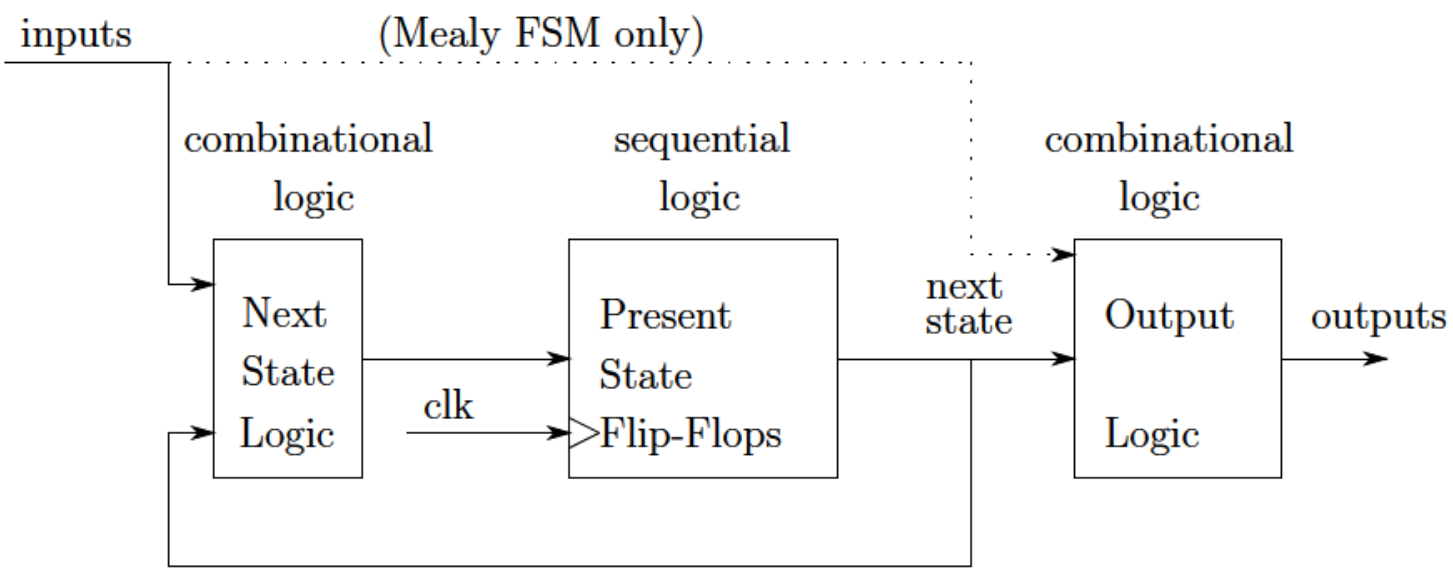

Figure 4.29: Mealy and Moore FSM. The next state logic is a combinational block (i.e. is comprised of combinational logic gates only) that determines which state in the FSM to transition into next. The present state flip-flops block is comprised of sequential logic that is updated at the next clock edge. The output logic block is a combinational logic block that considers the input(s) to the FSM only in a Mealy implementation. The Moore implementation is comprised of eveything in this diagram except for the dotted line that connects the inputs to the output logic block.

[22] is a paper well known for providing best practices for RTL coding. 


\subsubsection{Libraries}

Synthesis and $\mathrm{PNR}$ tools require timing and physical information about the standard cells in the library in order to implement the design. The timing information is in the form of tables that describe the transition time of the standard cell's output based on the capacitance the cell is driving. The delay of the cell is also included in this timing information. The cell's delay is determined from tables and is based on the transition time of the signal(s) driving the cell's input(s).

The physical information is in the form of the cell's input and output capacitance, the cell's physical size, and the location of the cell's pins.

\subsubsection{Constraints}

Synthesis and PNR tools require timing constraints in order to evaluate their resulting designs. Timing constraints consist of clock definitions. These definitions define the clock's period, duty cycle, rise and fall times, and the clock's uncertainties. The clock's uncertainties describe factors that effect the length of the clock's period. These factors can be any combination of cycle to cycle jitter, duty cycle distortion, on chip violation (OCV), and amount of allowable clock skew.

\subsection{Cycle to Cycle Jitter}

Cycle to cycle jitter is the worst case difference in clock periods between adjacent clock cycles.

\subsection{Duty Cycle Distortion}

Duty cycle distortion is the deviation from an ideal $50 \%$ duty cycle. The deviations can be positive, a duty cycle that is greater than $50 \%$, or negative, a duty cycle that is less than $50 \%$. 


\subsection{OCV}

OCV is a timing effect where the delay of a cell varies for the same cells placed at different locations on a die. What this means is that, for example, an INVX2 placed at two different locations on the same die, both driving the same capacitive load and both with an input signal with the same transition time each have different delays. The reason for differing cell delays is due to process, voltage, and temperature variations across the die.

\subsection{Allowable Clock Skew}

Ideally, a clock tree ensures that the clock arrives at the same time at all of the clock tree's endpoints. Unfortunately in practice, this isn't the case. The clock arrives at all of the clock tree's endpoints at different times. The difference in clock arrivals for the flip flop that launches the data and the clock arrival for the flip flop that captures the data is call clock skew. A value for the allowable clock skew is used in the clock uncertainty.

\subsubsection{PNR with Cadence Encounter}

The Cadence Encounter set of PNR tools can be used to create what is known as a floorplan. The floorplan is a physical description of the charge sharing SAR ADC?s layout. The floorplan defines the physical size of the SAR logic, the location of the input and output ports, the power grid's size, metal layer, and connectivity and the location of the rows used to place the standard cells.

The power grid is made up of power rings (located around the perimeter of the floorplan) and both vertical and horizontal power straps. Power is provided to the standard cells by connecting the ADC's package power and ground pins to the power grid. Current enters and exits via the package power and ground pins, then into and out of the power rings, and then into and out of the horizontal power and ground straps that connect to the standard cell's power and ground pins. The power grid must be designed so that the wires are wide enough so no electromigration occurs and also so that sufficient current 
can flow into the standard cells.

The power rings are routed on metal layer 3 horizontally and metal layer MQ vertically. The power rings are $4 \mu \mathrm{m}$ wide and spaced by $0.36 \mu \mathrm{m}$. The horizontal metal 1 power straps extend the entire length of the standard cell rows. Vertical power straps are also routed in metal layer $\mathrm{MQ}$ and are $4 \mu \mathrm{m}$ wide. These vertical straps are created in power and ground pairs that are spaced by $2 \mu \mathrm{m}$. The groups of power and ground straps are on a $30 \mu \mathrm{m}$ pitch.

The cells are placed in rows. The rows are created in what is known as "double back" where every even numbered row is flipped about the horizontal axis so that the power pins (named vdd!) of the standard cells face each other. This flipping of rows results in the ground pins of the standard cells (named gnd!) in two rows also face each other. The rows are spaced by $1 \mu \mathrm{m}$ to mitigate DRC violations such as minimum contact spacing rules.

As was previously described, clock skew is the difference in clock arrival times between the flip-flop launching the data and the flip-flop capturing the data. The ideal case is that this skew is zero. If the insertion delays of all the flip flops can be made the same, then the clock skew is zero. Therefore, a clock tree is built to try to match the insertion delay of all of the flip-flops. Unfortunately, the start point and end point flip flops in all the paths will separated by different physical distances. This makes balancing the insertion delay of all the paths very difficult. Therefore, it is common practice to determine an acceptable amount of insertion delay difference and use this value as a target when building a clock tree. Figure 4.30 shows an example of this timing path. The clock is applied to the clock pin of the charge sharing SAR ADC's package pin. The clock then travels through CLKINVX1 cells (these cells are shown in Figure 4.30 as a two buffers, buffer common $_{\text {and }}$ buffer $_{\text {s.p. }}$ ) to reach the clock pin of the flip-flop launching the data (commonly called the start point flop). Figure 4.30 shows that the clock travels through buffer ${ }_{\text {common }}$ and buffer $_{\text {e.p. }}$ (which are also comprised of multiple CLKINVX1 cells) before it reaches the clock pin of the flip-flop capturing the data (commonly called the end point flop). The time taken for the clock to travel from the clock package pin to the flip-flops it drives 
is called "insertion delay". Each flip-flop has a different insertion delay. The insertion

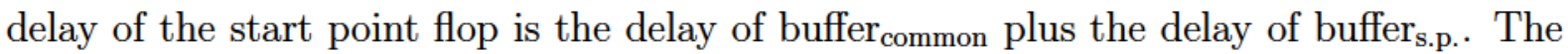
insertion delay of the end point flop is the delay of buffer common $_{\text {plus the delay of buffer }}$ e.p.

Figure ?? shows the SAR logic floorplan with the physical placement of the CLKINVX1 cells that make up the clock tree built in Cadence Encounter. The location of these CLKINVX1 cells isn't intuitive.

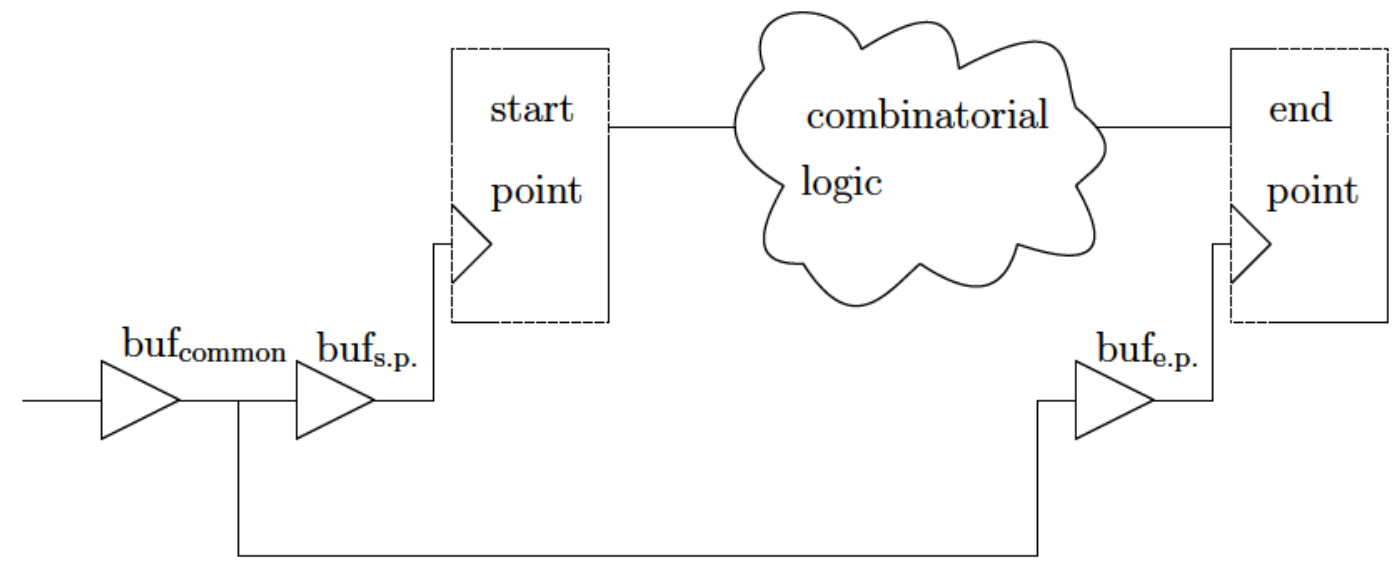

Figure 4.30: Illustration of register to register timing path. The start point flip-flop launches the data on the rising edge of the clock applied to it. The end point flip-flop captures the data on the rising edge of the clock applied to it. The difference between the arrival times of these two clocks is called clock skew. This is a single cycle timing path, the clock that triggers the end point flip-flop is one clock cycle later than the clock that triggers the start point flip-flop.

Cadence Encounter's signal router physically connects the placed input and output ports, and standard cell pins as defined in the structural Verilog imported from DC. The signal router also connects the clock tree cells. The signal router will do its best to route all nets without creating shorts or spacing violations. The process of routing begins by performing what is known as a "global route". Global route divides the floorplan into regions (known as route boxes) and then creates tracks (tracks are based on the routing pitch of each metal layer) used by the router to know where it can place routes. The global router is responsible for routing signals between these route boxes using the tracks on each metal layer. The global router only routes wires between these route boxes. It doesn't route to the level of the standard cell's pins or floorplan's ports. The detailed router is responsible for routing to and from the standard cell's pins or floorplan ports. 
The reason for using two routers is twofold, first, the designer can use the global router to determine the floorplan's routing congestion. Simply knowing if the space required to route all of the nets to connect all of the route boxes is greater than the area of the route boxes these nets must travel through lets the designer know if the floorplan needs adjustment. The second reason for using two routers is that routing algorithms are very complex, therefore, breaking the routing problem into two routers, and therefore, two routing algorithms makes the problem significantly less complex.

The ability to route designs using $\mathrm{PNR}$ tools such as Encounter does automate this task. However, the design will have to be modified or the designer will have to fix any shorts and or routes with spacing violations if the routers are unable to route all the nets without creating shorts and or spacing violations. The routing algorithms to date are not more intelligent than a designer. For the SAR logic, the router was restricted to only use metal layers 1 through MQ to route all nets. This left enough metal layers above the SAR logic for use to route between blocks at the SAR ADC level. Figure ?? shows the SAR logic after the design has been routed using Cadence Encounter.

The output of the flow is a gate level Verilog file and a DEF file. The gate level Verilog file output describes the interconnection of the standard cells that implement the algorithm as well as the design's clock tree. The DEF file describes the physical placement of the standard cells, the physical placement of the clock tree buffers, the physical locations of the routes that connect the standard cells and clock buffers, the physical locations of the input and output pins, and the physical locations of the power routes. 


\subsection{Sufficient current}

To ensure that sufficient current is provided to each of the standard cells in the layout, the standard cell power and ground pins are connected to the power grid not only at the East and West edges of the floorplan but also via vertical power straps.

Insufficient current provided to the standard cells degrades the cell's timing.

\subsection{Power grid verification}

To verify that the power grid design provides sufficient current, the logic's switching activity is provided. The reason why the switching activity is important is due to the fact that as the logic state of a standard cell transitions, it draws current from the power grid. If too much current is drawn from the power grid, the voltage at the power pins of the standard cells can drop below a minimum value, causing timing degradation. Cadence Encounter performs an IR drop analysis (common name for power grid verification) to determine the voltage at the various regions of the layout. If the voltage at any point in the layout is too low, the power grid can be modified in the following ways:

\subsection{Add more power metal}

Adding more power metal to the power grid increases the number of paths current can use to flow into the standard cells. This means that as the state of standard cell logic transitions, more current is available.

\subsection{Increase the number of VIAs}

VIAs are the interconnection between metal layers. Each VIA has a resistance value. Groups of VIAs are called "VIA Arrays". The resistance of a VIA array decreases as the number of VIAs in a VIA array increase. Therefore, decreasing the VIAs array's resistance, allows more current to flow into the standard cell's power pins as its output 
changes logic state.

\subsection{Add decoupling capacitors}

Decoupling capacitors act like resources of current as current is drawn from the power grid. Therefore, as the logic state of standard cells change, and draw current from the power grid, the decoupling capacitors reduce the effect of this current draw.

\subsubsection{DEF and Verilog import into Virtuoso}

The placed, routed, and timing closed layout using the Cadence Encounter PNR tool can be imported into Virtuoso for connection with the analog designs designed in the Virtuoso environment. To transfer a design from Encounter into Virtuoso, the DEF and Verilog files are written out of Encounter and then read into Virtuoso. Since the DEF describes the physical placement of the standard cells and routes, the layout view of the SAR logic is created when the DEF is imported into Virtuoso. The Verilog file describes the logical connectivity of the SAR logic, therefore, importing the Verilog file into Virtuoso generates the SAR logic's schematic view.

Each of the standard cells, INVX1, CLKINVX1, NAND2X1, NOR2X1, DFFRHQX1, and DFFSHQX1 have all passed DRC and layout versus schematic (LVS) checks individually. Therefore, when DRC checks are performed on the SAR logic, all that is really being checked is the routing performed in Encounter and that any routing errors that Encounter couldn't resolve have been fixed. SAR logic LVS checks verify that the logic implemented in the DEF matches the logic implemented in the DEF. This LVS check is used to ensure that no errors occurred during the transfer process from Encounter to Virtuoso.

It is important to note that the global power net was called vdd! and the global ground net was called gnd!. The global nets have an exclamation mark in their name so that the LVS tool can resolve the connection of these nets to cells that exist only in the layout, such as the "subc" cell. The subc cell is required to define the substrate 
connection of the NFET transistors in the IBM CMRF8SF library.

\subsubsection{Output pad driver}

The digital logic and analog circuits that implement the charge sharing SAR ADC are incapable of driving large capacitive loads such as bond pads and off chip loads. A pad driving buffer was developed based on the classic [23]. The pad driving buffer schematic is shown in Figure 4.31. The transistor sizes of this pad driver is shown in Table 4.3 .

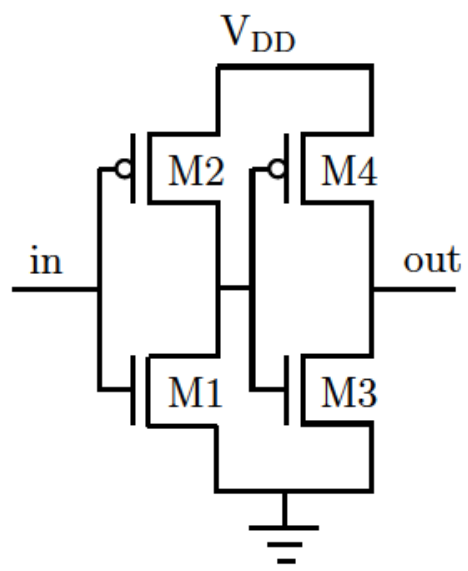

Figure 4.31: The schematic diagram of the pad driver. The driver is a pair of inverters that create a buffer. The sizes of the transistors is shown in Table 4.3 .

\begin{tabular}{|l|l|l|l|}
\hline Transistor & Width $(\mu \mathrm{m})$ & Length $(\mathrm{nm})$ & Number Fingers \\
\hline M1 & 3 & 120 & 4 \\
\hline M2 & 12 & 120 & 4 \\
\hline M3 & 16 & 120 & 16 \\
\hline M4 & 48 & 120 & 20 \\
\hline
\end{tabular}

Table 4.3: Transistor sizes for transistors used by pad driver shown in Figure 4.31 .

In order to verify the operation of this pad driving circuit, the test bench shown in Figure 4.32 was used. The signal source shown in Figure 4.32 drives a buffer with minimum sized transistors, which drives a wire modeled as a minimum sized MIMCAP from the IBM CMRF8SF library. The pad driver drives a bondpad. The bondpad cell used in the simulation was a schematic view of the wirebond bondpad cell from the IBM CMRF8SF library. The bondpad cell uses the same dimensions as the bondpad $(108 \mu \mathrm{m}$ 
$\mathrm{x} 108 \mu \mathrm{m})$ used in the layout. The bond pad drives a bond wire. It is assumed that the bond wire has an inductance of $2 \mathrm{nH}$. The sum of the bond wire's capacitance and the capacitance of a 'scope probe is estimated to be $15 \mathrm{pF}$. The components of this test bench is described in Table 4.4 .

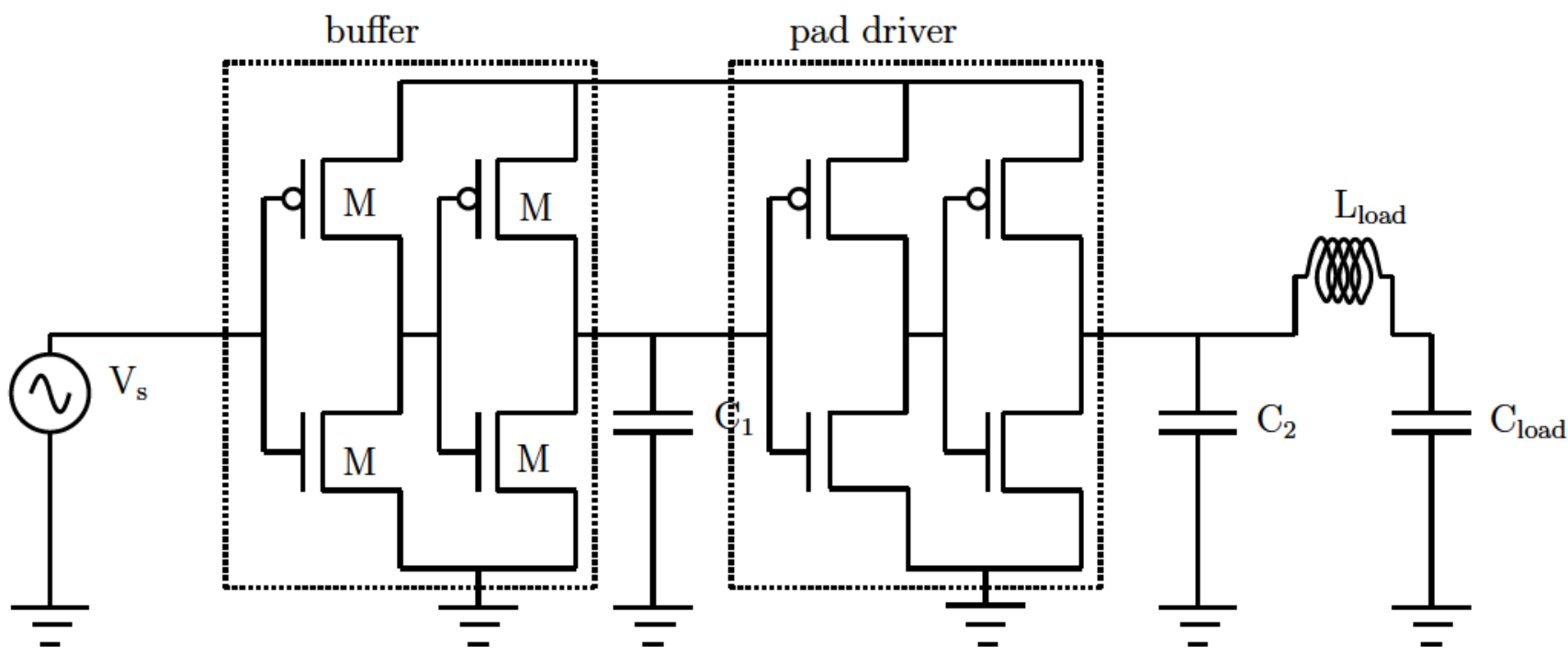

Figure 4.32: The schematic diagram of the pad driver's test bench. The test bench is comprised of a signal source that drives a buffer which drives the pad driver which drives a load. The components are described in Table 4.4

\begin{tabular}{|l|l|}
\hline Component & Component Description \\
\hline $\mathrm{V}_{\mathrm{s}}$ & $1 \mathrm{MHz}, 1.2 \mathrm{~V}$ square wave. \\
\hline $\mathrm{M}$ & Buffer transistors. width $=280 \mathrm{~nm}$, length $=120 \mathrm{~nm}$, fingers $=1$ \\
\hline $\mathrm{C}_{1}$ & $\begin{array}{l}\text { Capacitor to model an arbitrary wire connected to pad driver buffer. } \\
\text { Minimum sized IBM CMRF8SF MIMCAP }(153.4505 \mathrm{fF}) \text { used as a } \\
\text { model for an arbitrary wire. }\end{array}$ \\
\hline $\mathrm{C}_{2}$ & $108 \mu \mathrm{m} \times 108 \mu \mathrm{m}$ IBM CMRF8SF wirebond bondpad cell. \\
\hline $\mathrm{L}_{\text {load }}$ & Estimated bondwire inductance $(2 \mathrm{nH})$. \\
\hline $\mathrm{C}_{\text {load }}$ & Estimated bondwire and 'scope probe capacitance $(15 \mathrm{pF})$. \\
\hline
\end{tabular}

Table 4.4: Component description used in test bench shown in Figure 4.32 .

The signal applied by the testbench to the pad driver shown in Figure 4.32 and the output of the pad driver are shown in Figure 4.33. Figure 4.33 shows, the pad driver's output responds to the change in its input $4 \mathrm{~ns}$ after the input signal changes. This figure also shows that the pad driver's output reaches $1.2 \mathrm{~V} 15.237 \mathrm{~ns}$ after its input. 
NameVis

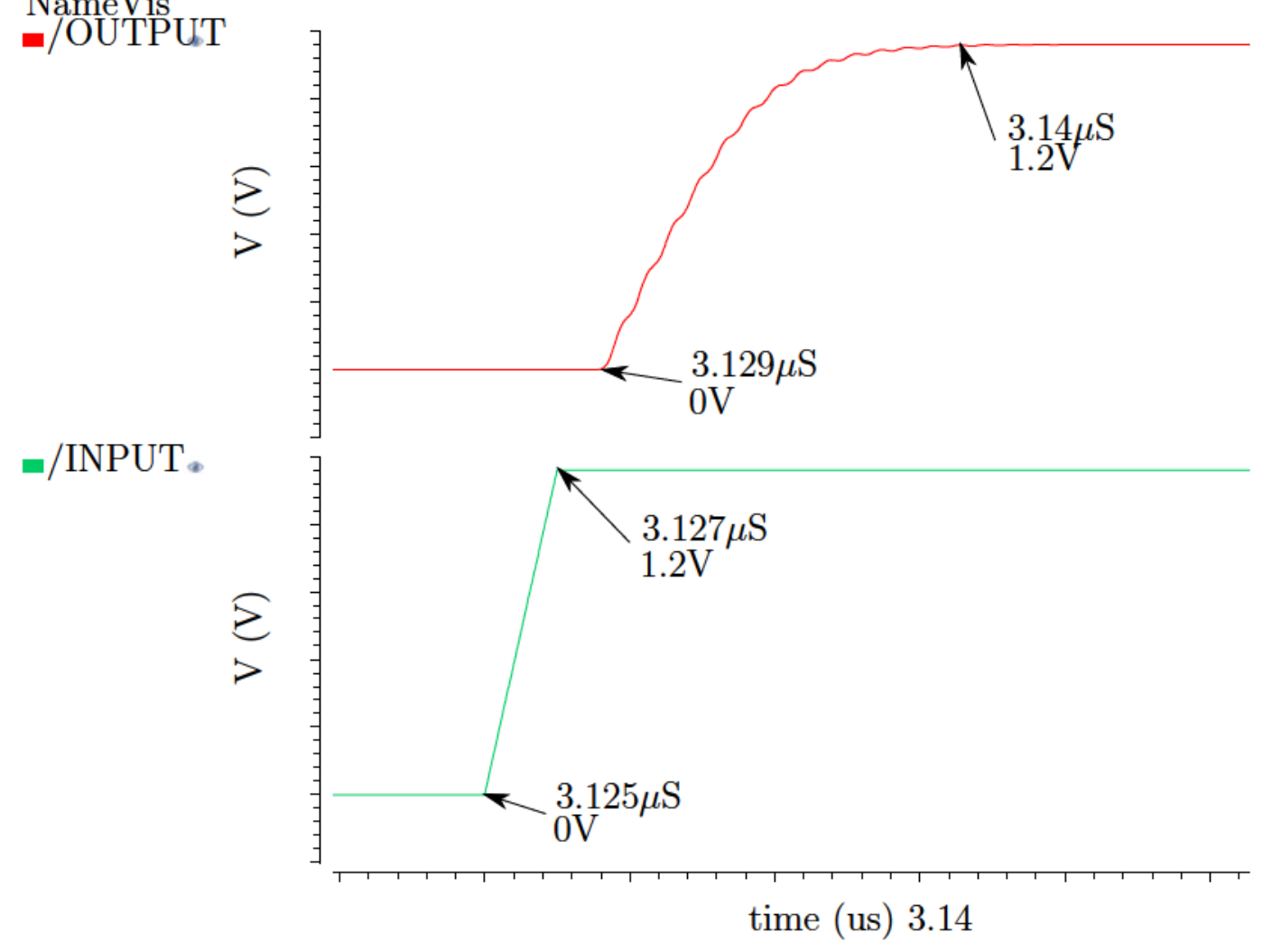

Figure 4.33: Zoomed in view of a single rising edge of the signal applied to the pad driver and its output from Figure 4.33. The pad driver's output responds to the change in its input 4ns after the input signal changes. This figure also shows that the pad driver's output reaches $1.2 \mathrm{~V} 15.237 \mathrm{~ns}$ after its input.

\subsubsection{ESD protection}

The IBM CMRF8SF library provides a double diode cell for use as an electrostatic discharge ESD protection device for transistors connected to input bondpads. This device consists of two diodes. The anode of the first diode is connected to the input pad and the cathode of this diode is connected to the positive power supply. A large positive ESD event forward biases this diode so that the ESD current flows into the positive power supply instead of into the transistors connected to the input pad. The cathode of the second diode of the double diode is connected to the input bondpad. The anode of this diode is connected to the negative power supply. Large negative ESD events forward bias this second diode so that the ESD current flow into the negative power supply instead on into the transistors connected to the input bondpad.

The IBM CMRF8SF library also provided an RC-ClampESD device. The RC-Clamp provides a path for currents from large positive and negative ESD events to flow from the 
positive power supply to the negative power supply instead of through any transistors connected to the positive power supply. 


\section{Chapter 5}

\section{Charge Sharing SAR ADC Simulation Results}

\subsection{Introduction}

This chapter describes the simulation methods and the simulation results of the charge sharing SAR ADC's functional blocks. The charge sharing SAR ADC's ENOB, SINAD, SNR, THD, SFDR, DNL, and INL are also discussed. The chapter concludes with pfet and nfet transistor leakage simulations.

\subsection{Comparator Monte Carlo Verification}

The StrongARM comparator generates an output that is either $0 \mathrm{~V}$ or $1.2 \mathrm{~V}$. The value is based on the voltage difference applied between its negative and positive input terminals. For example, $1.2 \mathrm{~V}$ is output when the voltage applied to the comparator's positive input is greater than the voltage applied to its negative input. $0 \mathrm{~V}$ is output when the voltage applied to the comparator's negative input is greater than the voltage applied to its positive input.

This comparator is a clocked comparator, therefore, the value of its inputs determine the value of its output only when the clock transitions from $0 \mathrm{~V}$ to $1.2 \mathrm{~V}$ (the clock's rising edge). The comparator's output remains at this voltage regardless of the voltage 
difference applied to its inputs, until, the clock returns to $0 \mathrm{~V}$. When the clock is $0 \mathrm{~V}$, the comparator's output is forced to 0V regardless of the voltage difference applied to it's input terminals.

The comparator's output is used in two ways. Its first use is as the value of each bit of the charge sharing ADC's output word. The second is as an input to the SAR digital logic.

The Charge Sharing SAR ADCPs digital output word is comprised of 8-bits. Each bit of this 8-bit word is actually the voltage generated by the comparator at each rising edge of the clock. Therefore, it takes 8 clock cycles to create the 8 -bits required for the ADCs 8-bit word.

The comparator's output is also used by the SAR digital logic as a factor in determining the next voltage generated by the capacitor array.

The StrongARM comparator must be able to detect a voltage difference that is at most one LSB of the charge sharing $[\mathrm{SAR}$ ADC]s digital output word. Since this ADC]s output word is 8 -bits, the voltage of one $\mathrm{LSB}$, assuming a reference voltage of $1 \mathrm{~V}$, is $3.90625 \mathrm{mV}$.

The StrongARM comparator must be able to determine this $3.90625 \mathrm{mV}$ difference in the following two cases.

1) When one input is minimum $(0 \mathrm{~V})$ and the other is at most $3.90625 \mathrm{mV}$. The transient waveforms used to verify the StrongARM comparator in this case are shown in Figure 5.1. Figure 5.1 shows the waveforms for eight tests performed under these conditions. The description of each of these eight tests is contained in Table 5.1. 


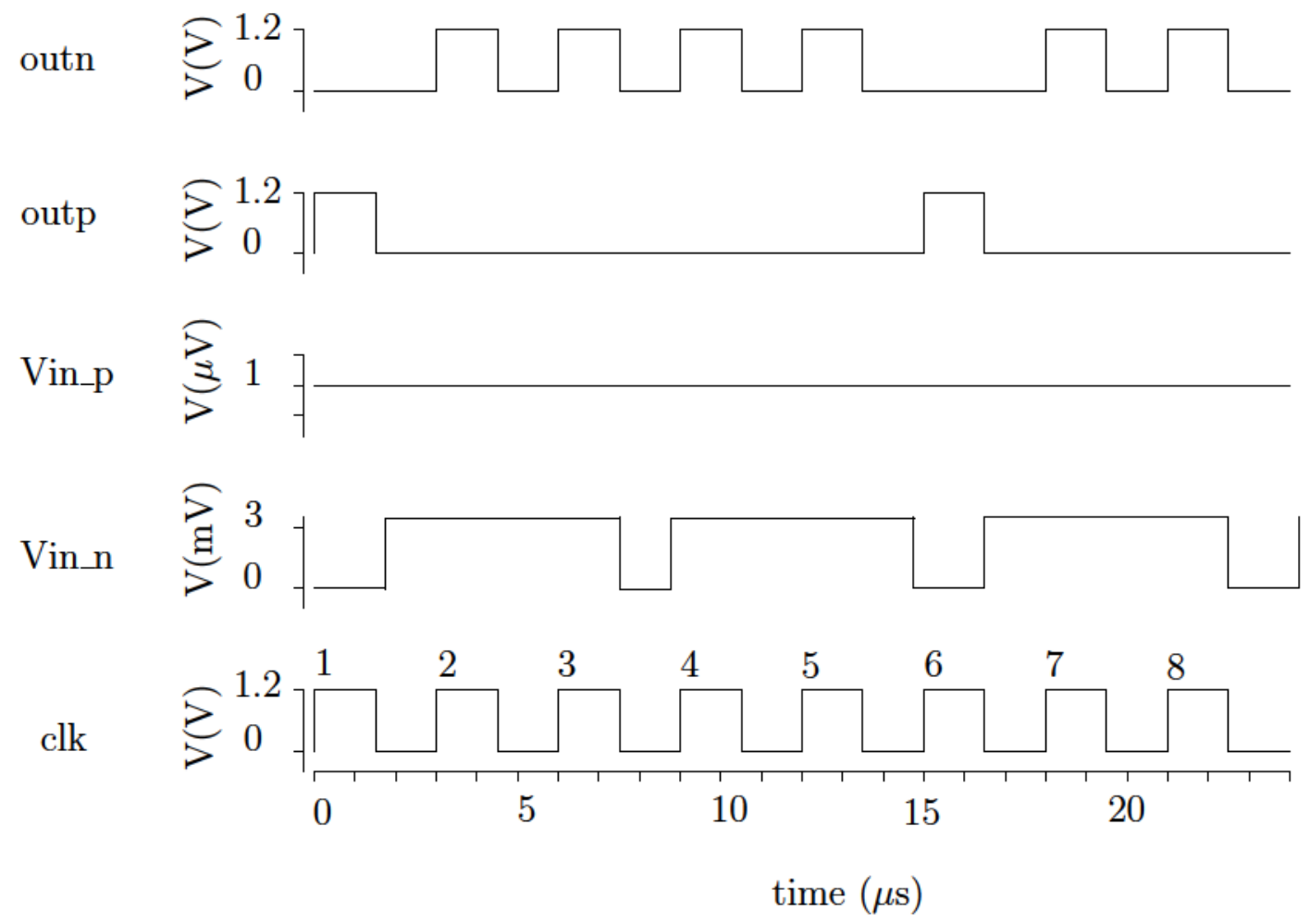

Figure 5.1: Verify operation of StrongARM comparator when $3.90625 \mathrm{mV}$ applied to Vin_n input and $1 \mu \mathrm{V}$ applied to Vin_p input. At clock rising edges 1 and 6, the voltage applied to Vin p input is greater than the voltage applied to Vin_n input, resulting in a pulse being generated on the comparator's positive output (outp). At clock rising edges $2,3,4,5,7$, and 8, the voltage applied to input Vin_n is greater than the voltage applied to the Vin_p input which causes the comparator's negative output (outn) to pulse. 


\begin{tabular}{|c|c|}
\hline Clock Edge & Test Description \\
\hline 1 & No test. \\
\hline 2 & Vin_n set to $3.90625 \mathrm{mV} 1.4625 \mu$ s before rising clock edge 2 . \\
\hline 3 & $\begin{array}{l}\text { Vin_n is set to } 3.90625 \mathrm{mV} \text { for more than one clock cycle } \\
(4.639975 \mu \mathrm{s}) \text { before rising clock edge } 3 \text {. }\end{array}$ \\
\hline 4 & $\begin{array}{l}\text { Vin } n \text { is set to } 0 \mathrm{~V} 1.7725 \mu \text { s before rising clock edge } 4 \text { but transitions } \\
\text { to } 3.90625 \mathrm{mV} 0.102409 \mu \text { s before rising clock edge } 4 \text {. This test shows } \\
\text { that the comparator is unable to detect signal levels that transition } \\
0.102409 \mu \text { s prior to the rising clock edge. This is proved due to } \\
\text { the fact that the comparator positive output transitions and the } \\
\text { negative output does not. It was found that the comparator can } \\
\text { not detect signal transitions, at nominal PVT, for times less than } \\
\text { or equal to this value through simulation experiments. }\end{array}$ \\
\hline 5 & $\begin{array}{l}\text { Simple test to determine if the comparator can correctly detect } \\
\text { that its positive output (outp) should be pulsed when Vin_n is set } \\
\text { to } 3.90625 \mathrm{mV} \text { at least one clock cycle (in this case } 3.22489 \mu \mathrm{s} \text { ) prior } \\
\text { to, and close to one clock cycle (in this case } 2.7125 \mu \mathrm{s} \text { ) after the } \\
\text { clock rising edge following the test where it has not been able to } \\
\text { detect a signal transition (test at rising clock edge } 4 \text { ). }\end{array}$ \\
\hline 6 & $\begin{array}{l}\text { Minimum setup time test. This test shows that the comparator can } \\
\text { detect signal levels that transition } 0.41 \mu \text { s prior to the rising clock } \\
\text { edge. This was proved by the comparator's positive output pulsing } \\
\text { in response to the voltage level of the comparator's positive input } \\
\text { being greater than its negative input. This was found to be the } \\
\text { minimum setup time for the comparator, at nominal[PVT, through } \\
\text { simulation experiments. }\end{array}$ \\
\hline 7 & $\begin{array}{l}\text { Simple test to determine if the comparator can detect signal tran- } \\
\text { sitions half a clock cycle prior to the rising edge of the clock. }\end{array}$ \\
\hline 8 & $\begin{array}{l}\text { Simple test to determine if the comparator can detect signal tran- } \\
\text { sitions one and a half clock cycles prior to the rising edge of the } \\
\text { clock. }\end{array}$ \\
\hline
\end{tabular}

Table 5.1: Description of comparator tests shown in Figure 5.1. 
2) When one input is full scale (1V) and the other is at least $3.90625 \mathrm{mV}$ less than 1V (996.09375mV). The transient waveforms used to verify the StrongARM comparator in this case are shown in Figure 5.2 . Figure 5.2 shows the waveforms for eight tests performed under these operating conditions. A description of each of these eight tests, and their results, are contained in Table 5.2 .

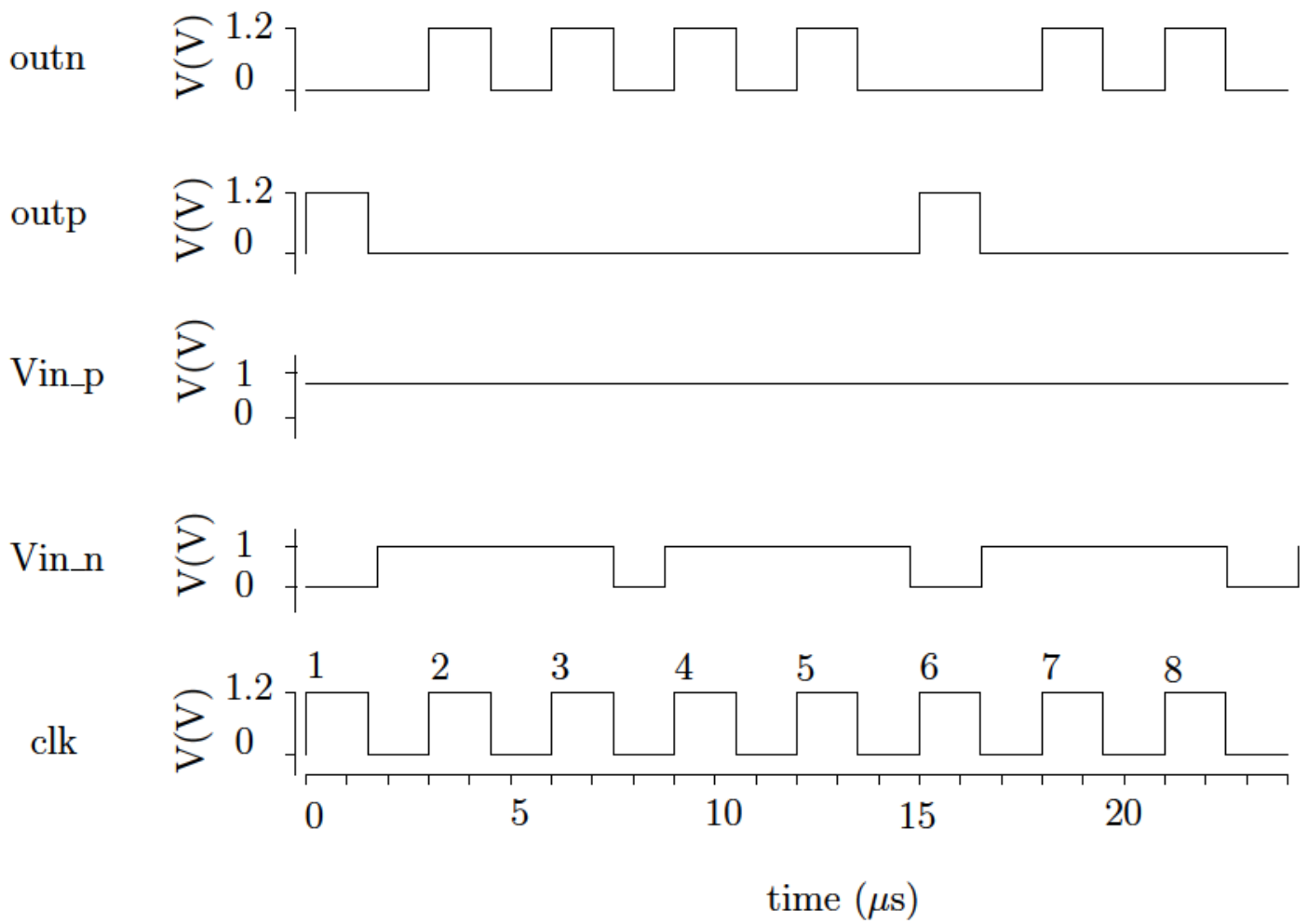

Figure 5.2: Verify operation of StrongARM comparator when $1 \mathrm{~V}$ applied to Vin_n input and $996.094 \mathrm{mV}$ applied to Vin_p input. At clock rising edges 1 and 6 the voltage applied to Vin_p input is greater than the voltage applied to Vin_n input resulting in a pulse being generated on the positive comparator output (outp). At clock rising edges 2, 3, 4, 5, 7, and 8, the voltage applied to input Vin_n is greater than the voltage applied to the Vin_p input which causes the negative comparator output (outn) to pulse. 


\begin{tabular}{|c|c|}
\hline Clock Edge & Test Description \\
\hline 1 & No test. \\
\hline 2 & Vin_n set to $1 \mathrm{~V} 1.4625 \mu$ s before rising clock edge 2 . \\
\hline 3 & $\begin{array}{l}\text { Vin } n \text { is set to } 1 \mathrm{~V} \text { for more than one clock cycle }(4.639975 \mu \mathrm{s}) \text { before } \\
\text { rising clock edge } 3 \text {. }\end{array}$ \\
\hline 4 & $\begin{array}{l}\text { Vin } n \text { is set to } 0 \mathrm{~V} 1.7725 \mu \text { s before rising clock edge } 4 \text { but transi- } \\
\text { tions to } 1 \mathrm{~V} 0.072278 \mu \text { s before rising clock edge } 4 \text {. This test shows } \\
\text { that the comparator is unable to detect signal levels that transition } \\
0.072278 \mu \text { s prior to the rising clock edge. This is proved due to } \\
\text { the fact that the comparator positive output transitions and the } \\
\text { negative output does not. This was found to be the minimum time } \\
\text { that the comparator can not detect signal transitions, at nominal } \\
\text { PVT, through simulation experiments. }\end{array}$ \\
\hline 5 & $\begin{array}{l}\text { Simple test to determine if the comparator can correctly detect that } \\
\text { its positive output (outp) should be pulsed when Vin_n is set to } 1 \mathrm{~V} \\
\text { for at least one clock cycle (in this case } 3.225 \mu \mathrm{s} \text { ) prior to, and close } \\
\text { to one clock cycle (in this case } 2.7125 \mu \mathrm{s} \text { ) after the clock rising edge } \\
\text { when it has not been able to detect a signal transition (test at rising } \\
\text { clock edge } 4 \text { ). }\end{array}$ \\
\hline 6 & $\begin{array}{l}\text { Minimum setup time test. This test shows that the comparator } \\
\text { can detect signal levels that transition } 0.41001 \mu \text { s prior to the rising } \\
\text { clock edge. This was proved by the comparator's positive output } \\
\text { pulsing in response to the voltage level of the comparator's positive } \\
\text { input being greater than its negative input. This was found to } \\
\text { be the minimum setup time for the comparator, at nominal PVT, } \\
\text { through simulation experiments. }\end{array}$ \\
\hline 7 & $\begin{array}{l}\text { Simple test to determine if the comparator can detect signal tran- } \\
\text { sitions half a clock cycle prior to the rising edge of the clock. }\end{array}$ \\
\hline 8 & $\begin{array}{l}\text { Simple test to determine if the comparator can detect signal tran- } \\
\text { sitions one and a half of a clock cycles prior to the rising edge of } \\
\text { the clock. }\end{array}$ \\
\hline
\end{tabular}

Table 5.2: Description of comparator tests shown in Figure 5.2 .

The best case, nominal[PVT, simulation waveforms are shown in Figure 5.1 and Figure 5.2. These figures show the waveforms generated by the comparator in response to the stimulus when the capacitance driven by both sides of the comparator's differential pair are equal. Unfortunately, the manufacturing process is imperfect and the fabricated device's differential pair won't drive equal capacitances. Simulations prove that a suffi- 
ciently large capacitor imbalance results in the comparator making an incorrect decision. What this means is that the comparator's response to the stimulus as shown in Figure 5.1 and Figure 5.2 will be incorrect and won't be the same as shown in these two figures. To overcome this capacitor mismatch, the comparator is calibrated by adding capacitance, via complementary metal oxide semiconductor (CMOS) switches, to one side of the comparator's differential pair. Monte Carlo analysis was performed to quantitatively measure the effectiveness of calibration when the stimulus waveforms shown in both Figure 5.1 and Figure 5.2 are run 200 times varying both process and mismatch parameters. This analysis uses device models provided by IBM with statistical distributions representing long-term process variations observed during manufacturing [24]. The results of these 200 Monte Carlo runs are shown in Figure 5.3 .

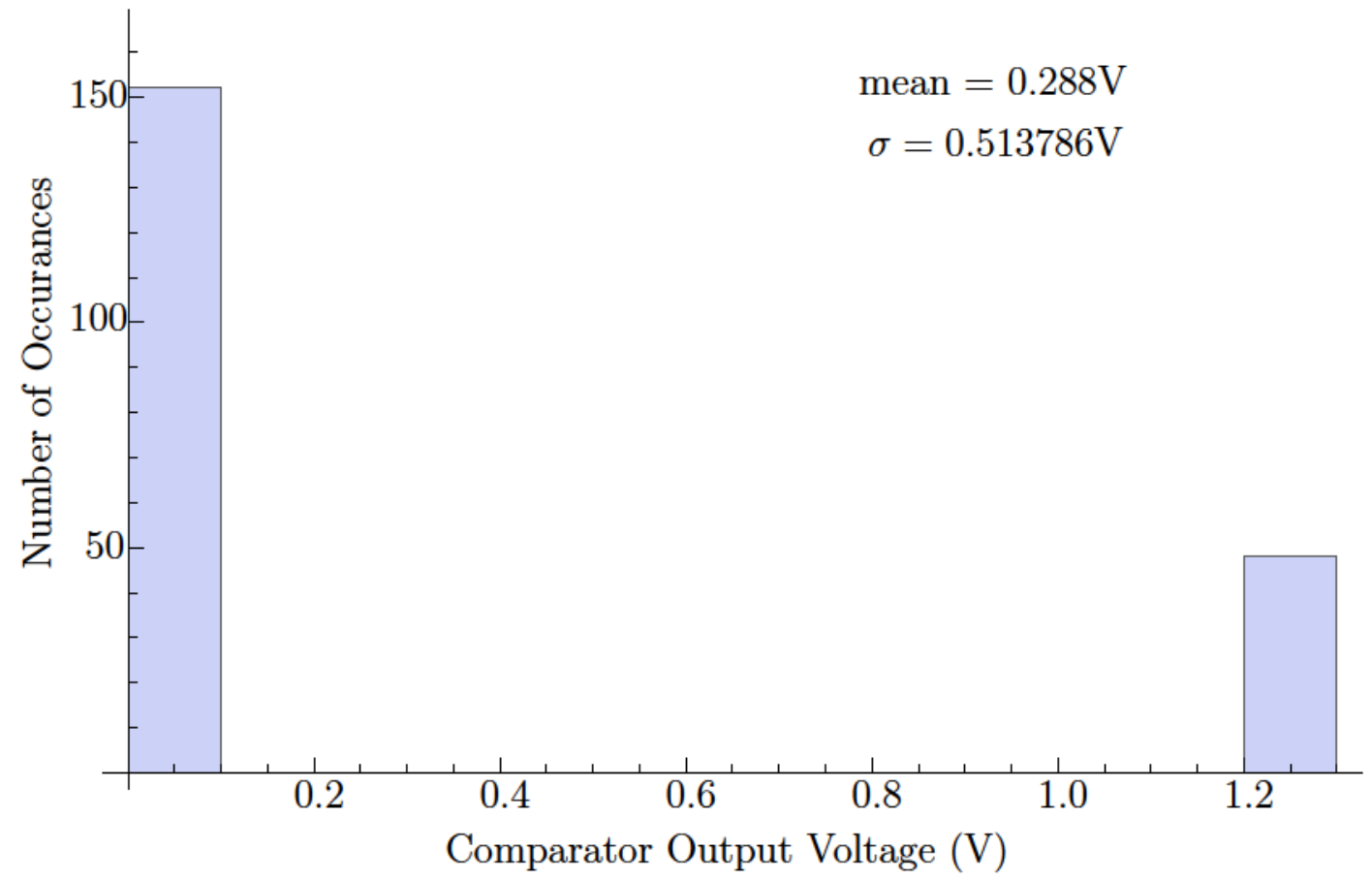

Figure 5.3: Monte Carlo analysis of StrongARM comparator with $V_{\text {in_n }}$ set to $1 \mu \mathrm{V}$ and $V_{\text {in_p }}$ set to $3.90625 \mathrm{mV}$ as shown in Figure 5.1. The test shown in Figure 5.1 was repeated 200 times with process and mismatch statistical variation for each test. This analysis was performed before calibration. The negative comparator output should be $1.2 \mathrm{~V}$ at rising clock edges $2,3,4,5,7$, and 8 . The positive comparator output should be $1.2 \mathrm{~V}$ at rising clock edges 1 and 6 . The transient waveforms for each of the 200 tests were analyzed. The comparator output is only $1.2 \mathrm{~V}$ when both negative and postive comparator outputs match the waveforms shown in Figure 5.1. The comparator didn't generate the correct output 152 times so it is incorrect $76 \%$ of the time.

As Figure 5.3 shows, $76 \%$ of the time, the comparator makes the wrong decision of which output to pulse when the comparator is tested using Figure 5.1. The results of 
the 200 Monte Carlo runs of test Figure 5.2 are shown in Figure 5.4. This time however, Figure 5.4 shows that the comparator incorrectly decided which output to pulse $4 \%$ of the time.

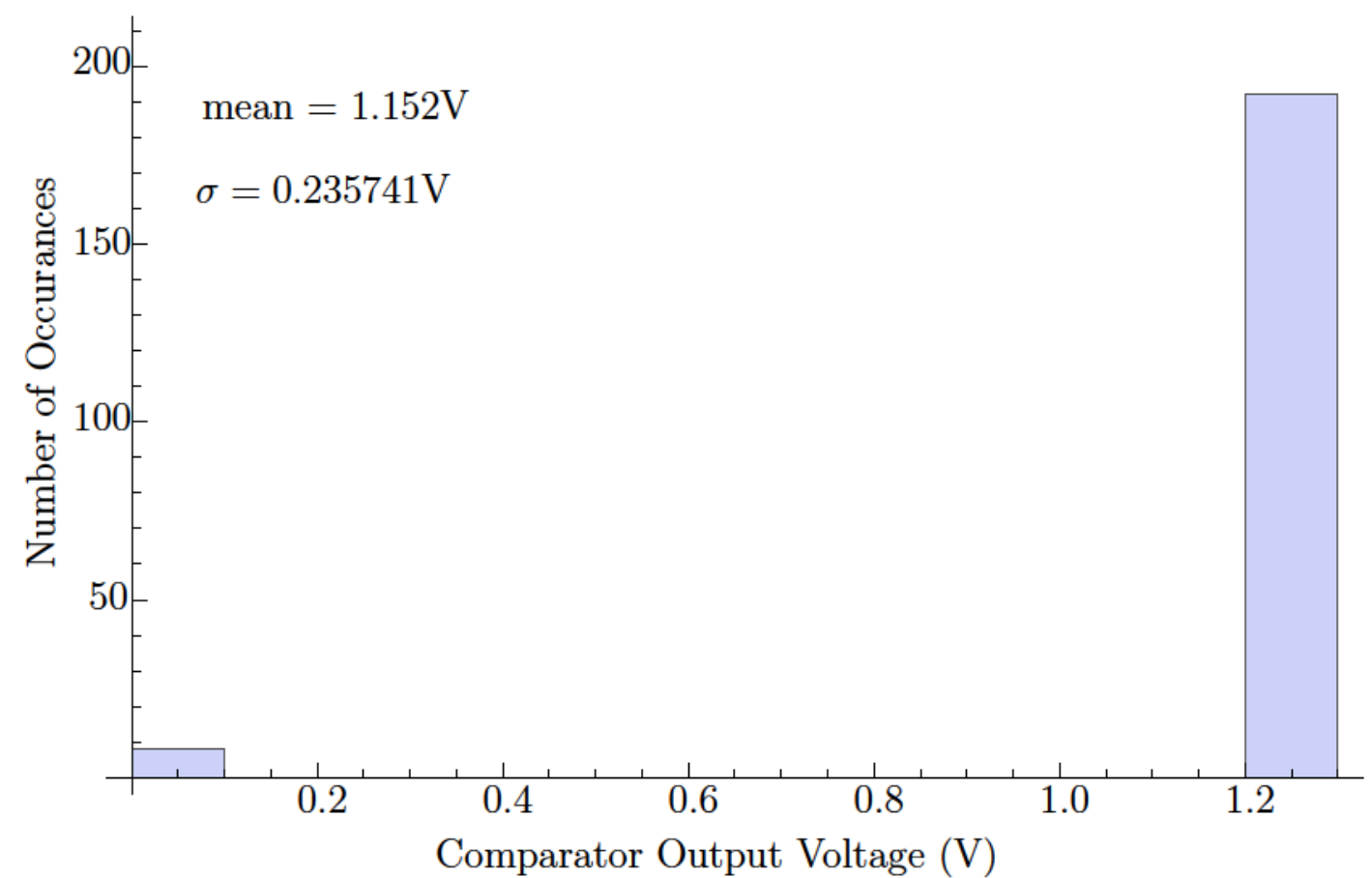

Figure 5.4: Monte Carlo analysis of StrongARM comparator with $\mathrm{V}_{\text {in_n }}$ set to $1 \mathrm{~V}$ and $\mathrm{V}_{\text {in_p }}$ set to $0.996094 \mathrm{~V}$ as shown in Figure 5.2. The test shown in Figure 5.2 was repeated 200 test points with process and mismatch statistical variation for each test. This analysis was performed before calibration. The negative comparator output should be $1.2 \mathrm{~V}$ at rising clock edges $2,3,4,5,7$, and 8 . The positive comparator output should be $1.2 \mathrm{~V}$ at rising clock edges 1 and 6 . The transient waveforms for each of the 200 tests were analyzed. The output should be $1.2 \mathrm{~V}$ when both negative and postive comparator outputs match the waveforms shown in Figure 5.2. The comparator didn't generate the correct output 8 times so it is incorrect $4 \%$ of the time.

In simulation, the comparator's calibration was validated for all 200 Monte Carlo runs using the eight tests shown in Figure 5.1. Each Monte Carlo run has different circuit parameters and each of these 200 sets of circuit parameters were saved as a "corner" in the Cadence GXL simulation environment. Then each "corner" was used during a transient simulation to create 200 transient simulations. For each of these 200 "corners", the comparator calibration was carried out by either adding capacitance until the transient analysis matched the waveforms shown in Figure 5.1 or until the maximum capacitance of the calibration circuit was reached.

The second step of this calibration validation was to perform 200 Monte Carlo analyses using the eight tests shown in Figure 5.2 and saving each set of circuit parameters as a 
"corner". These "corners" resulted in 200 transient simulations performed using the same calibration settings as the 200 transient simulations for the tests shown in Figure 5.1. Only two of these 200 transient simulations under these conditions didn't match the results of tests shown in Figure 5.2 .

The calibrated comparator Monte Carlo analysis results are shown in Figure 5.5 . Calibration has improved the comparator's decision making so that it only makes the incorrect decision $22.5 \%$ of the time for both tests from Figure 5.1 and Figure 5.2 .

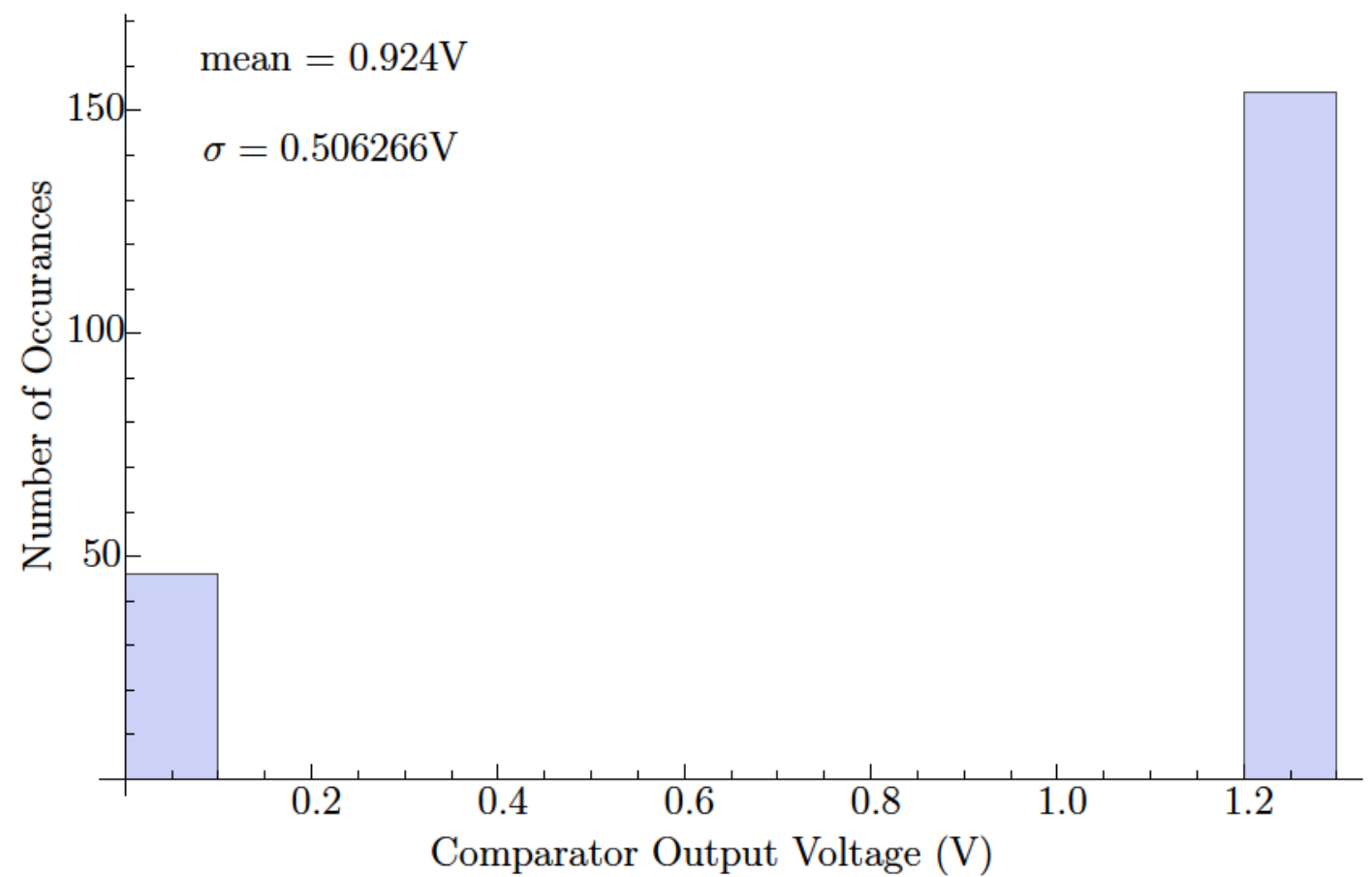

Figure 5.5: Monte Carlo analysis of StrongARM comparator post calibration. The comparator was calibrated for test Figure 5.1 repeated 200 times with process and mismatch statistical variation. The comparator retained the same calibration and test Figure 5.2 was repeated 200 times with process and mismatch statistical variation. The comparator's output should be $1.2 \mathrm{~V}$ for all 200 tests, but, as this figure shows, the comparator's output is incorrect 45 times or $22.5 \%$ of the time.

Since the StrongARM comparator is a clocked comparator, changes in its inputs are only detected when the comparator's clock transitions from a logic low to a logic high. It is usual for the evaluation of a comparator's performance to generate the comparator's analog transfer function. To generate the analog transfer function, an increasing ramp is applied to one of the comparator's inputs and a decreasing ramp is applied to the comparator's other input. The voltage difference between the two input ramps that causes the output to transition is the interesting point to observe from the transfer function. However, since the clocked comparator only detects changes in its inputs when the 
comparator's clock transitions from a logic low to a logic high, the transfer function for a clocked comparator isn't as interesting. Therefore, it is more valuable for a clocked comparator to analyze the variance of the clocked comparator's threshold voltage using Monte Carlo process and mismatch simulations.

The StrongARM comparator's output is determined based on the current difference flowing through the comparator's differential pair. The threshold voltage of the differential pair's transistors is affected by process and mismatch variation. This shifting threshold voltage difference between the the differential pair shifts the threshold of the comparator and appears as an offset voltage. To measure this offset voltage, Monte Carlo process and mismatch simulations were performed and the results are shown in Figure 5.6 . Figure 5.6 shows that offset voltage varies within $3 \sigma$ which is acceptable for a fabricated device.

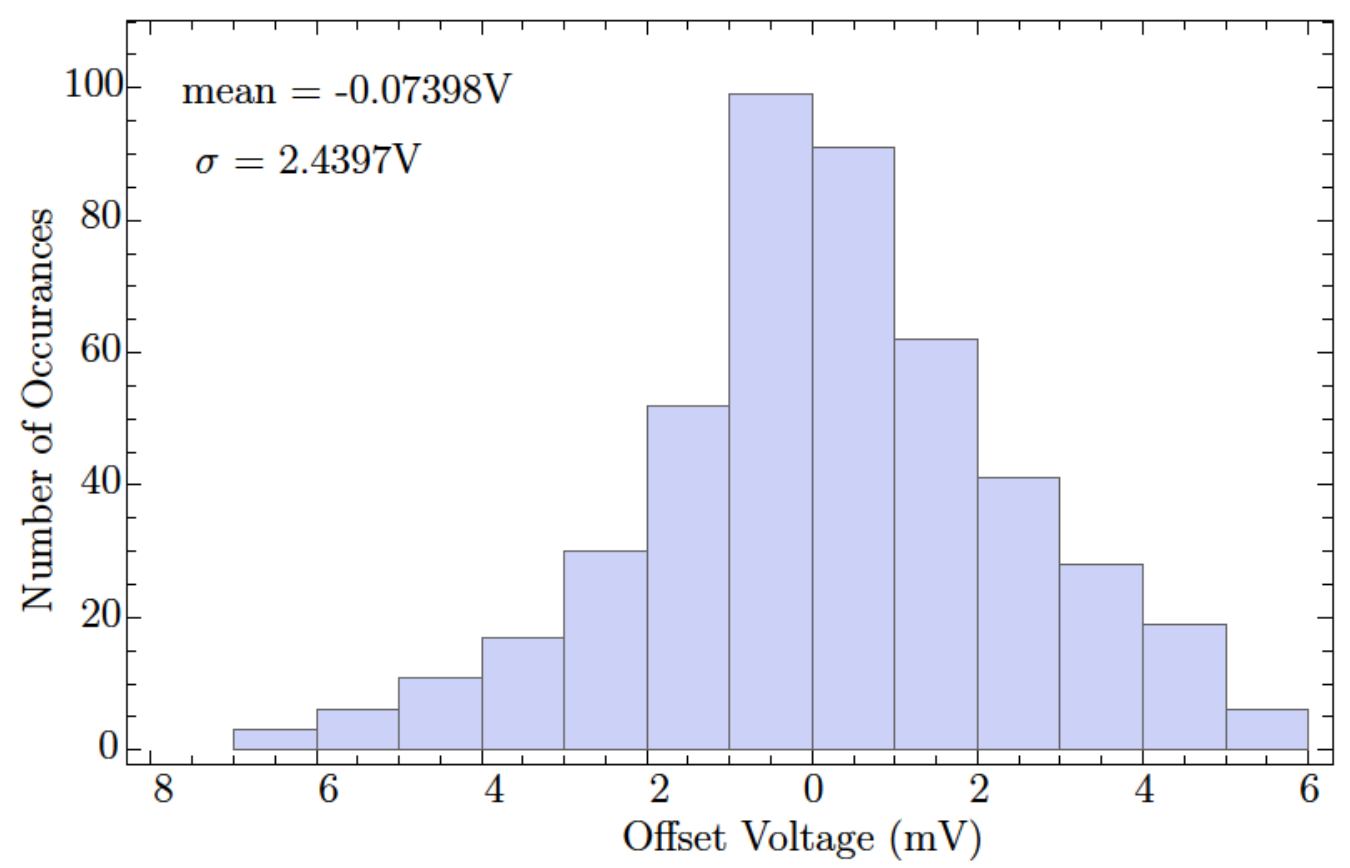

Figure 5.6: Monte Carlo analysis of StrongARM comparator's offset voltage. 600 process and mismatch simulations were performed and as the figure shows, the extremes of the offset voltage remains within $3 \sigma$. 


\subsection{Sample and Hold Simulated Performance}

A transient analysis of the $\mathrm{S} / \mathrm{H}$ is shown in Figure 5.7. As Figure 5.7 shows, a $1 \mathrm{kHz}$ sinusoid is applied to the $\mathrm{S} / \mathrm{H}$ 's input and a $20 \mathrm{kHz}$ sampling clock is used. The frequencies of interest for telemonitoring systems are less than $1 \mathrm{kHz}$. The frequency of the sampling clock must meet at least the minimum requirements of the Nyquist-Shannon sampling theorem. According to the Nyquist-Shannon sampling theorem, a sampled signal can be perfectly reconstructed (i.e. not aliased) as long as the frequency of the sampled signal is not greater than half of the sampling period. The Nyquest-Shannon sampling theorem is mathematical and assumes a perfect sampling signal and sampling conditions. Therefore, because this $\mathrm{S} / \mathrm{H}$ won't operate in perfect sampling conditions, the frequency of the sampling signal was increased significantly from this minimum requirement and is 20 times the input signal.

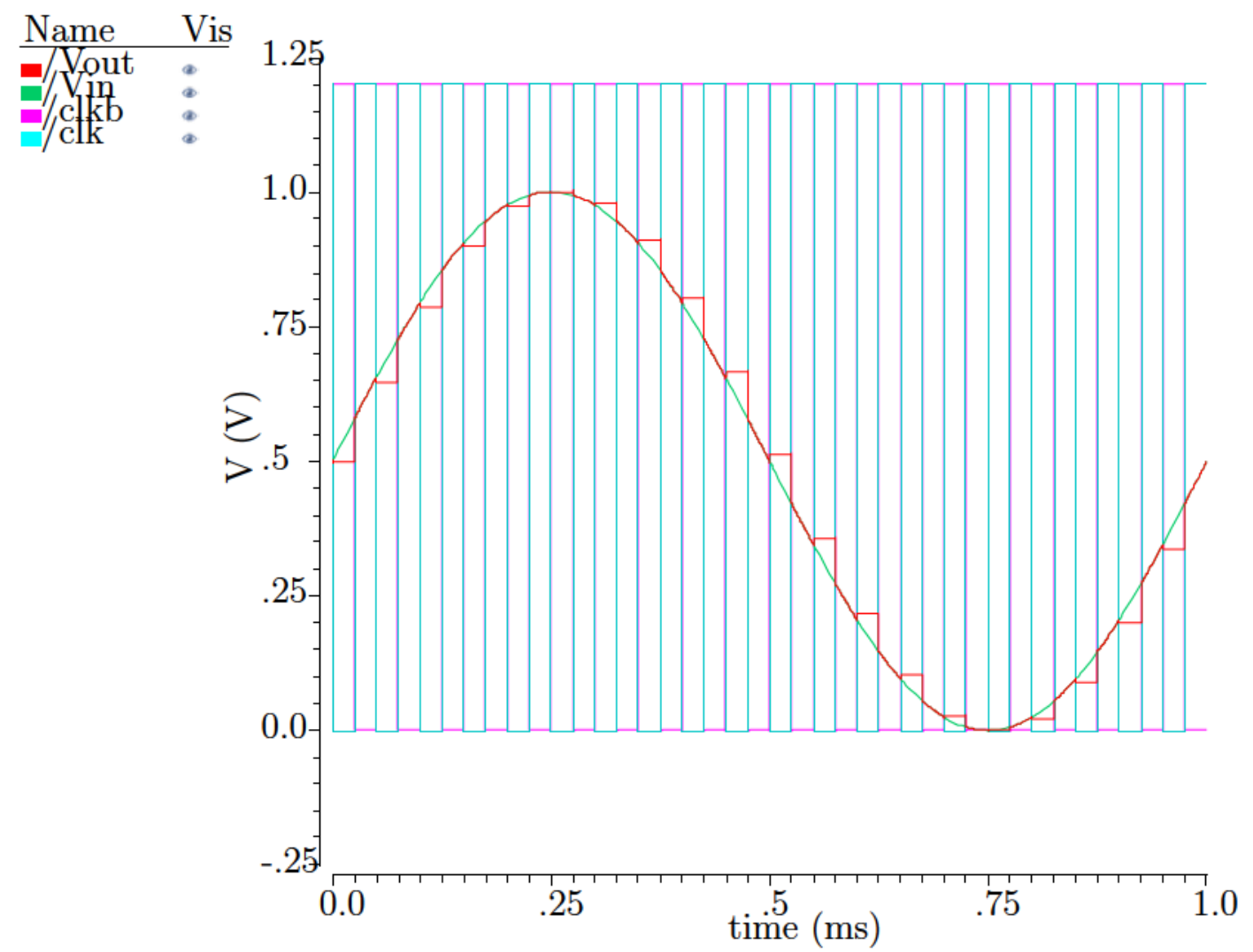

Figure 5.7: Transient analysis of $\mathrm{S} / \mathrm{H}$. The input signal is a $1 \mathrm{kHz}$ sine wave and the sampling clocks (clk and clkb) both operate at $20 \mathrm{kHz}$. The output of the $\mathrm{S} / \mathrm{H}$ when in sampling mode (the sampling clock, clk is high and clkb is low) follows the input signal. The output of the $\mathrm{S} / \mathrm{H}$ when in hold mode (the sampling clock, clk is low and clkb is high) retains the value of the input signal when sampled.

The performance of a $\mathrm{S} / \mathrm{H}$ is measured using several metrics. These metrics are 
settling time, hold pedestal, droop, SNR, SINAD, ENOB, THD, and SFDR.

When the $\mathrm{S} / \mathrm{H}$ changes from simply sampling the signal applied to its input to holding the sampled input signal, the transistor switch that applies the input signal to the capacitor storing the signal opens. This causes a transient and it takes a certain period of time for the voltage across the capacitor to reach a steady state. This time is known as the "settling time". Settling time is measured as the time taken for the voltage across the capacitor (or in this case the output voltage of the $\mathrm{S} / \mathrm{H}$ ) to reach $0.1 \%$ of the voltage applied to the $[\mathrm{S} / \mathrm{H}] \mathrm{s}$ input $[25]$. The settling time was measured to be $753.6 \mathrm{~ns}$ as shown in Figure 5.8 .

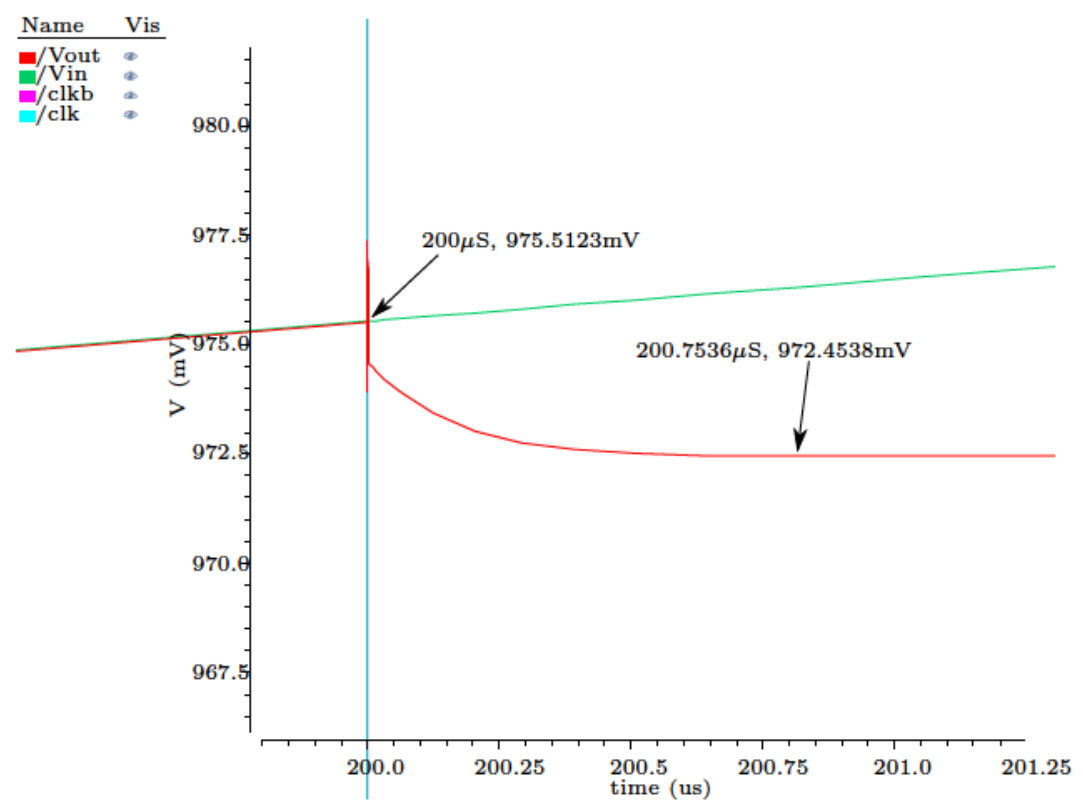

Figure 5.8: Transient analysis of $\mathrm{S} / \mathrm{H}$ showing the settling time and hold pedestal. The input signal is sampled at $200 \mu$ s with an amplitude of $975.5123 \mathrm{mV}$ and settles to steady voltage of $972.4538 \mathrm{mV}$ at $200.7536 \mu$ s. The settling time is therefore $753.6 \mathrm{~ns}$. The hold pedestal is $3.0585 \mathrm{mV}$.

Figure 5.9 shows the largest voltage transition of the $\mathrm{S} / \mathrm{H}$ s output voltage as a result of the $\mathrm{S} / \mathrm{H}$ entering hold mode. This transition is very fast compared to the setting time since it lasts for only $2.5 \mathrm{~ns}$ where the settling time is measured to be $753.6 \mathrm{~ns}$.

Hold pedestal is a dc offset voltage resulting from the transistor(s) in the switch that apply the input signal to the capacitor turn off which allows their channel charge to modify the voltage stored across the capacitor. This offset voltage is measured by subtracting the voltage output by the $\mathrm{S} / \mathrm{H}$ after the $\mathrm{S} / \mathrm{H} / \mathrm{s}$ output has settled (i.e. after settling time) and the voltage input to the $\mathrm{S} / \mathrm{H}]$ when the input is sampled [12]. Figure 5.8 


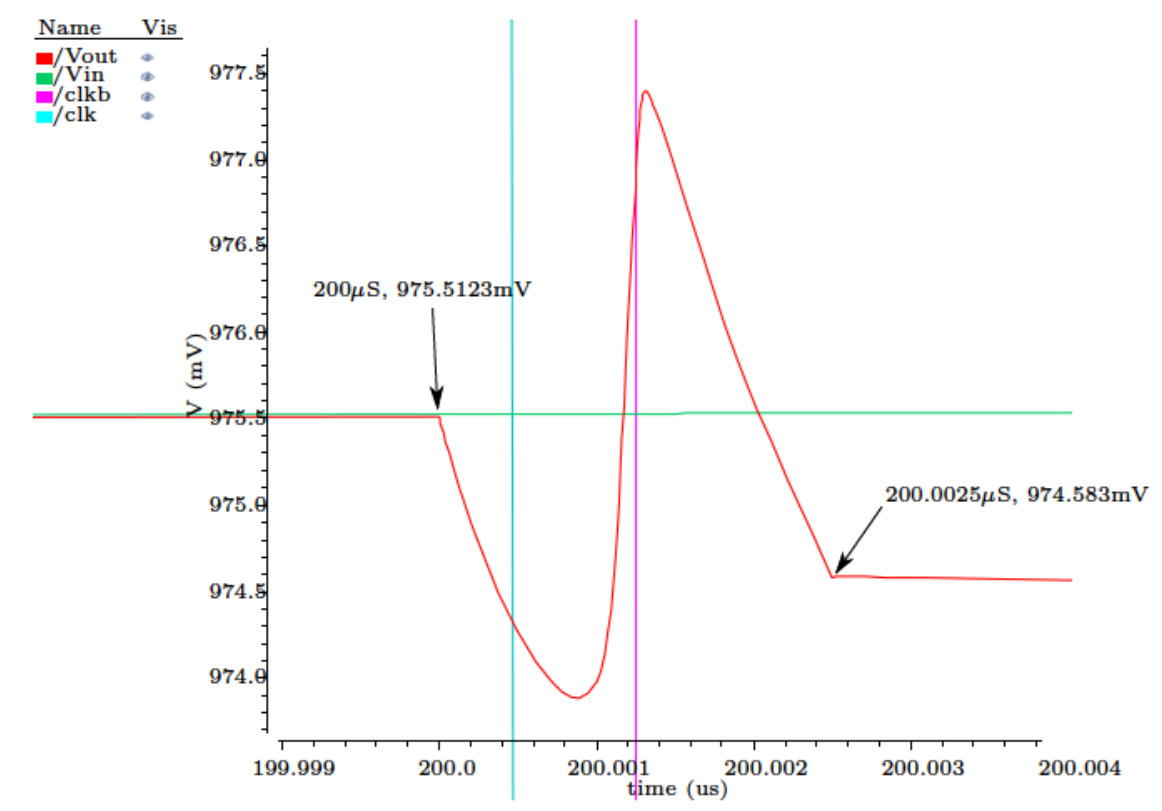

Figure 5.9: Transient analysis of $\mathrm{S} / \mathrm{H}$ showing a zoomed in view of the largest voltage transition of the $\mathrm{S} / \mathrm{H}$ output voltage when the $\mathrm{S} / \mathrm{H}$ enters into hold mode.

shows the measured hold pedestal to be $3.0585 \mathrm{mV}$.

While the $\mathrm{S} / \mathrm{H}$ is in hold mode, the ideal situation is that the voltage across the capacitor (and output by the $\mathrm{S} / \mathrm{H}$ ) remain constant. Unfortunately, leakage currents resulting from reverse-biased junctions allow the capacitor to discharge slowly over time. Commonly, the resulting change in voltage stored by the capacitor due to these leakage currents are insignificant and therefore ignored [12]. The signals sampled in this application are very low frequency and therefore the voltage stored across the capacitor must be held for a long period of time. This means that voltage droop is a significant concern. One method to mitigate voltage droop is to increase the size the of the capacitor that stores the sampled voltage. The second method to reduce voltage droop is the reduction of channel charge applied to the capacitor by the transistor switch using a clock with long rise and fall times. The minimum sized VNCAP was used (680.1726fF) to reduce the amount of area required to implement the $\mathrm{S} / \mathrm{H}$ and the rise and fall times of the clock were set to $2.5 \mathrm{~ns}$. Therefore, the rise and fall times and capacitance were traded off to reduce both area and charge injection.

The transient simulation was performed on the $\mathrm{S} / \mathrm{H}$ to determine its voltage droop. The $\mathrm{S} / \mathrm{H}$ output and sampling clock waveforms are shown in Figure 5.10. As Figure 5.10 shows, that while the $\mathrm{S} / \mathrm{H}$ is operating in hold mode that the voltage droop is not mea- 
surable. At $1.009175 \mu$ s the output voltage is measured to be $499.9597 \mathrm{mV}$. At $25.002 \mu \mathrm{s}$ the output voltage is measured to be $500.01 \mathrm{mV}$. The input signal is a $1 \mathrm{kHz}$ sine wave and the clock is a $20 \mathrm{kHz}$ square wave. As the measurements indicate, due to the speed of the sampling clock relative to the frequency of the input signal and the size of the sampling capacitor, there is no voltage droop.

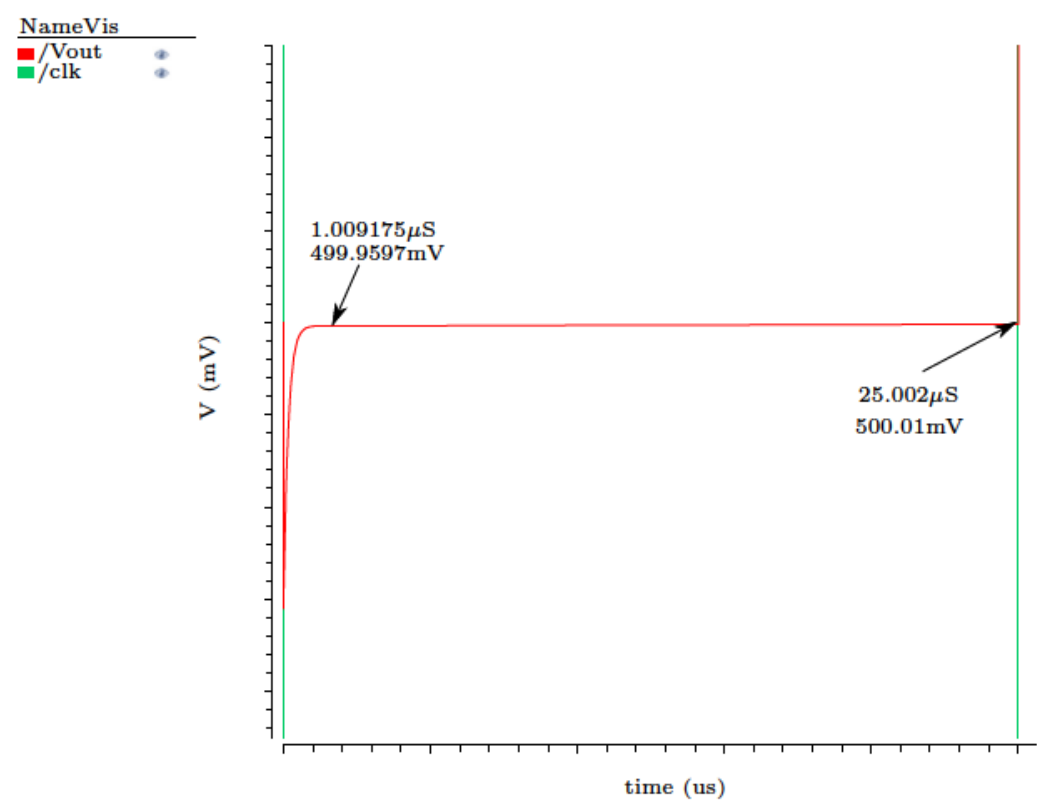

Figure 5.10: Transient analysis of $\mathrm{S} / \mathrm{H}$ showing the output voltage of the $\mathrm{S} / \mathrm{H}]$ while the $\mathrm{S} / \mathrm{H}$ is operating in hold mode. At $1.009175 \mu$ s the output voltage is measured to be $499.9597 \mathrm{mV}$. At $25.002 \mu$ s the output voltage is measured to be $500.01 \mathrm{mV}$. The input signal is a $1 \mathrm{kHz}$ sine wave and the clock is a $20 \mathrm{kHz}$ square wave. As the mesauements indicate, due to the speed of the sampling clock relative to the frequency of the input signal and the size of the samping capacitor, there is no voltage droop.

$\mathrm{SNR}$ is defined as the ratio of the signal power to the noise power and is calculated using Equation 5.1 ,

$$
S N R=10 \log _{10}\left(\frac{P_{\mathrm{C}}}{\sum_{i=1}^{N} P h_{\mathrm{i}}-P_{\mathrm{C}}}\right)
$$

where, $\mathrm{P}_{\mathrm{C}}$ is the power of fundamental frequency extracted from the $\mathrm{S} / \mathrm{H}$ s output, $\mathrm{Ph}_{\mathrm{i}}$ is the $i$ th harmonic power frequency from the $\mathrm{S} / \mathrm{H}$. s output, and $\mathrm{N}$ is the number of samples.

SINAD is the ratio of the root mean squared $(\overline{R M S})$ value of the input signal to the RMS noise measured at the $\mathrm{S} / \mathrm{H}$ 's output. To calculate SINAD Equation 5.2 is 
substituted into Equation 5.3 [26] can be used,

$$
\text { noise }_{\mathrm{RMS}}=\sqrt{\frac{1}{M} \sum_{n=1}^{M}\left(y_{n}-y_{n}^{\prime}\right)^{2}}
$$

where, $\mathrm{M}$ is the number of harmonics, $\mathrm{n}$ is the variable with value between 1 and $\mathrm{M}$, $\mathrm{y}_{n}$ is the sample data set, $\mathrm{y}_{n}^{\prime}$ is the data set of the best fit sine wave,

$$
S I N A D=\frac{\text { signal }_{\mathrm{RMS}}}{\text { noise }_{\mathrm{RMS}}}
$$

where, signal ${ }_{\mathrm{RMS}}$ is the (peak amplitude of input)/ $\sqrt{2}$.

As [26] describes, the method to calculate SINAD using Equation 5.3 requires the application of a sinusoid to the $\mathrm{S} / \mathrm{H}$ s input. This sinusoid must have what $[26$ describes as "good short term stability" so that it has no distortion nor random noise as these effects can degrade the $\mathrm{S} / \mathrm{H}$ s performance. The next step in the SINAD calculation is to fit the measured noise at the $\mathrm{S} / \mathrm{H}$ s output to a sinusoid at the fundamental frequency as per section 4.1 .4 of 26 , and then calculate the $\mathrm{S} / \mathrm{H}$ s SINAD using Equation 5.3 .

ENOB is SINAD expressed in terms of bits instead of in terms of decibels. Equation 5.4 can be used to calculate ENOB. Equation 5.4 is defined in [26],

$$
E N O B=\log _{2}(\mathrm{SINAD})-\frac{1}{2} \log _{2}(1.5)-\log _{2}\left[\frac{A}{(V / 2)}\right]
$$

where, $\mathrm{A}$ is the amplitude of input sine wave fitted to the output, and V is the full-scale range of device under test.

THD of a signal is defined as the amount of harmonic distortion present and is the ratio of the sum of all harmonic frequency power to the power of the fundamental frequency. 
Equation 5.5 is used to calculate THD,

$$
T H D=20 \log _{10}\left(\frac{\sqrt{\sum_{h}\left(X_{\mathrm{avm}}\left(f_{h}\right)\right)^{2}}}{\sqrt{\left(X_{\mathrm{avm}}\left(f_{i}\right)\right)^{2}+\left(X_{\mathrm{avm}}\left(f_{s}-f_{i}\right)\right)^{2}}}\right)
$$

where, $\mathrm{X}_{\mathrm{avm}}\left(f_{h}\right)$ is the averaged magnitude of the component at the $h$ th harmonic of the fast fourier transform (FFT) of the $\mathrm{S} / \mathrm{H}$ s output signal, $\mathrm{X}_{\mathrm{avm}}\left(f_{i}\right)$ is the averaged magnitude of the output fundamental component at the signal test frequency, and $\mathrm{X}_{\mathrm{avm}}\left(f_{s}-f_{i}\right)$ is the averaged magnitude of the output frequency that is the difference between the sampling frequency and the fundamental component at the signal test frequency.

The definition of SFDR is the ratio of the fundamental to the strongest spurious signal output by the $\mathrm{S} / \mathrm{H}$. 26] defines that $\mathrm{SFDR}$ is calculated as the ratio of the fundamental frequency in the $\mathrm{S} / \mathrm{H}$ s output to the amplitude of either the largest harmonic or spurious frequency component in the $\mathrm{S} / \mathrm{H}$; s output over the entire Nyquist band. 26] also defines spurious frequency components as persistent sine waves at frequencies other than the harmonic frequencies. The equation used to calculate SFDR is shown in Equation 5.6 ,

$$
S F D R=20 \log _{10}\left(\frac{\left|\mathrm{X}_{\mathrm{avg}}\left(f_{i}\right)\right|}{\max _{f_{s}, f_{h}}\left\{\left|X_{\mathrm{avg}}\left(f_{h}\right)\right| \operatorname{or}\left|X_{\mathrm{avg}}\left(f_{s}\right)\right|\right\}}\right)
$$

where, $\mathrm{X}_{\text {avg }}$ is the averaged spectrum output by the $\mathrm{S} / \mathrm{H}, \mathrm{f}_{i}$ is the amplitude of the input signal frequency, $\mathrm{f}_{h}$ is the amplitude of the harmonic spectral component frequency, and $\mathrm{f}_{s}$ is the amplitude of the spurious spectral component frequency.

A transient analysis was performed as a part of the determination of the $\mathrm{S} / \mathrm{H}$ s $\mathrm{s}$ performance. The simulation applied an analog signal to the $\mathrm{S} / \mathrm{H}$ s input and also applied a clock signal. The resulting $[\mathrm{S} / \mathrm{H}$ output and applied analog input, and clock signals were written out of Cadence and then read into Matlab. The Matlab code performed a FFT] and then calculated SNR, SINAD, ENOB, THD, and SFDR, A 960Hz sine wave was applied to the input of the $\mathrm{S} / \mathrm{H}$ and a $20 \mathrm{kHz}$ square wave was applied to the $\mathrm{S} / \mathrm{H}$ 's 
clock input.

The $\mathrm{S} / \mathrm{H}$ s performance in the typical nfet/typical pfet $(\mathrm{TT})$ and $27^{\circ} \mathrm{C}$, fast corner, fast nfet/fast pfet $(\overline{F F})$ and $-40^{\circ} \mathrm{C}$, and slow nfet/slow pfet $(\mathrm{SS})$ and $125^{\circ} \mathrm{C}$ are shown in Figure 5.11, Figure 5.12, and Figure 5.13 respectively. Theses figures show not only the fundamental frequency but also its harmonics and intermodulation frequencies. The harmonic frequencies are a result of the S/H's output not being a sinusoidal waveform. While the S/H is in "hold" mode, the S/H's output is a "flat spot". This is "flat spot" is a D.C. voltage that the rest of the ADC uses to converge on an equivalent digital value. This "flat spot" causes harmonic frequencies that are integer multiples of the fundamental. The intermodulation frequencies are a result of charge injection from the transmission gate as it turns off. This charge injection are carries, either electrons or holes, that made up the transmission gate's channel. This charge flows from the transmission gate when it turns off. The charge injection modifies the charge stored on the S/H's capacitor, causing distortion and generating frequencies other than the fundamental of its harmonics.

The SNR, SINAD, ENOB, THD, and SFDR measurements at these three process points and operating temperatures are summarized in Table 5.3 . 
The $\overline{\mathrm{S} / \mathrm{H}}$ s performance in the TT process point (typical nfet and typical pfet) at $27^{\circ} \mathrm{C}$ is shown in Figure 5.11 .

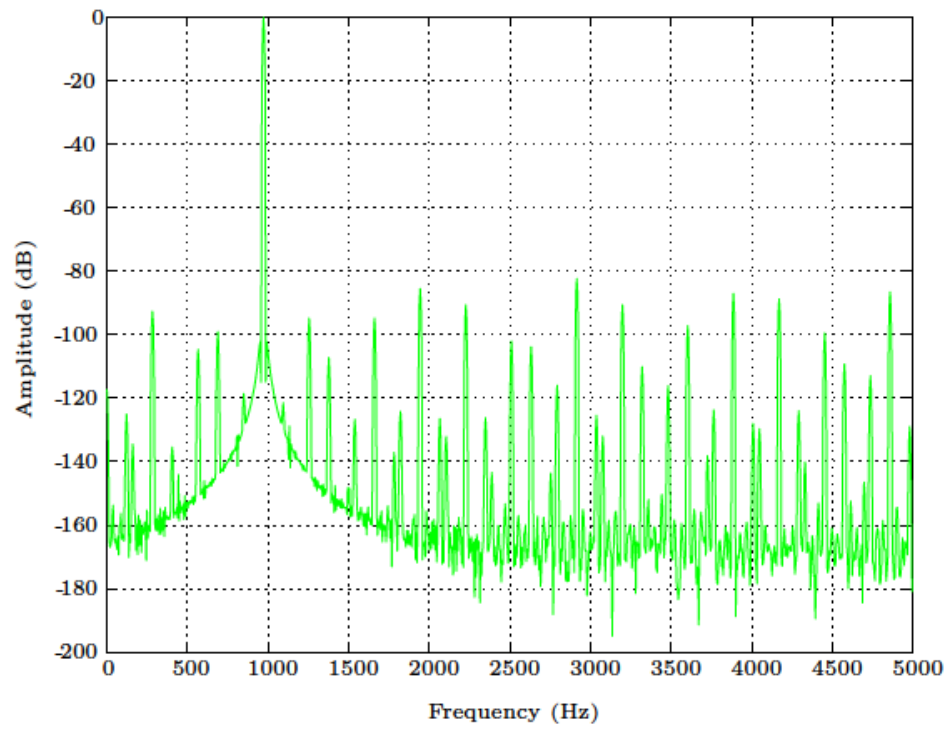

Figure 5.11: $\mathrm{S} / \mathrm{H}$ frequency spectrum generated using Matlab. This spectrum was used to measure $\mathrm{S} / \mathrm{H}$ /s performance for TT process and $27^{\circ} \mathrm{C}$. The measured $\mathrm{SNR}=80.1 \mathrm{~dB}, \mathrm{SINAD}=77.5 \mathrm{~dB}$, ENOB $=12.6 \mathrm{bits}, \mathrm{THD}=-80.9 \mathrm{~dB}$, and $\mathrm{SFDR}=84.7 \mathrm{~dB}$. A $960 \mathrm{~Hz}$ sine wave was applied to the input of the $[\mathrm{S} / \mathrm{H}$ and a $20 \mathrm{kHz}$ square wave was applied to the $\mathrm{S} / \mathrm{H}$ 's clock input. Therefore, the $960 \mathrm{~Hz}$ sinusoid was sampled 21 times.

The $\mathrm{S} / \mathrm{H}$ s performance using the $\mathrm{FF}$ corner at $-40^{\circ} \mathrm{C}$ is shown in Figure 5.12 .

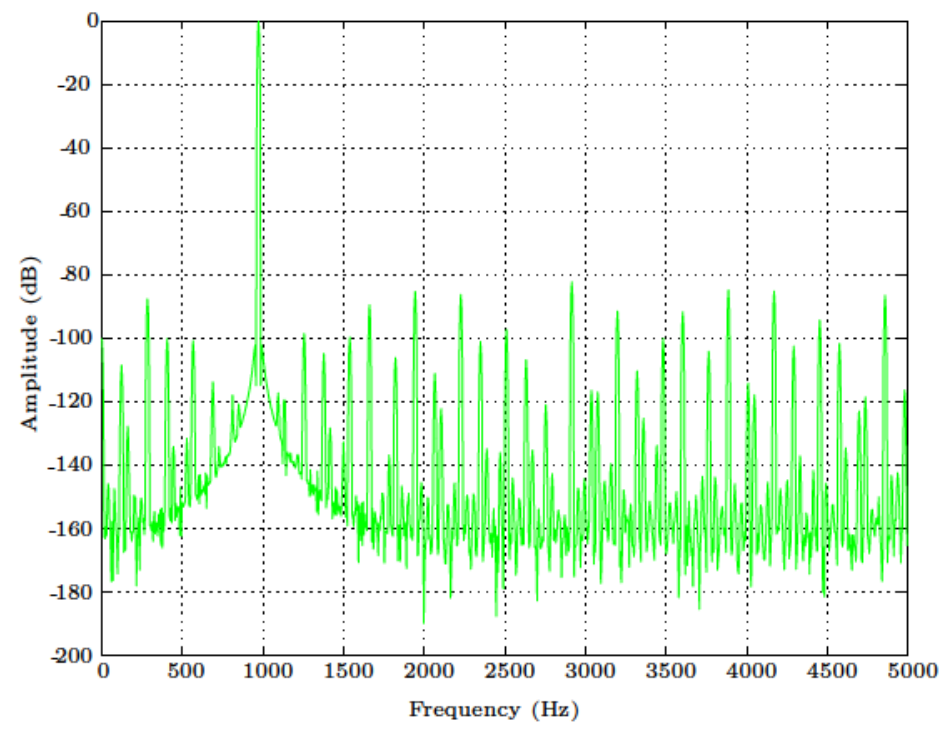

Figure 5.12: $\mathrm{S} / \mathrm{H}$ frequency spectrum generated using Matlab. This spectrum was used to measure $\mathrm{S} / \mathrm{H}$ 's performance for $\mathrm{FF}$ process and $-40^{\circ} \mathrm{C}$. The measured $\mathrm{SNR}=78.1 \mathrm{~dB}, \operatorname{SINAD}=76.0 \mathrm{~dB}$, ENOB $=12.3 \mathrm{bits}, \mathrm{THD}=80.1 \mathrm{~dB}$, and $\mathrm{SFDR}=84.8 \mathrm{~dB}$. A $960 \mathrm{~Hz}$ sine wave was applied to the input of the $[\mathrm{S} / \mathrm{H}$ and a $20 \mathrm{kHz}$ square wave was applied to the $\mathrm{S} / \mathrm{H}$ 's clock input. Therefore, the $960 \mathrm{~Hz}$ sinusoid was sampled 21 times. 
The $\mathrm{S} / \mathrm{H}$ s performance using the $\mathrm{SS}$ corner at $+125^{\circ} \mathrm{C}$ is shown in Figure 5.13 .

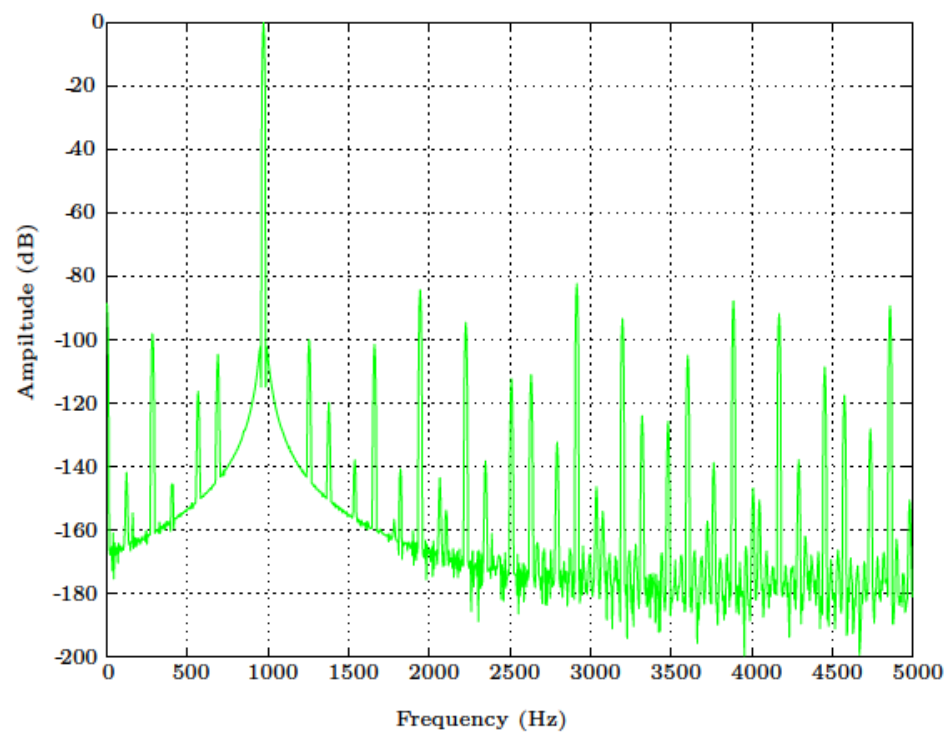

Figure 5.13: $\mathrm{S} / \mathrm{H}$ frequency spectrum generated using Matlab. This spectrum was used to measure $\mathrm{S} / \mathrm{H}$ ]s performance for SS process and $+125^{\circ} \mathrm{C}$. The measured $\mathrm{SNR}=81.0 \mathrm{~dB}$, SINAD $=78.1 \mathrm{~dB}$, ENOB $=12.7$ bits, $T H D=81.3 \mathrm{~dB}$, and $\operatorname{SFDR}=84.8 \mathrm{~dB}$. A $960 \mathrm{~Hz}$ sine wave was applied to the input of the $[\mathrm{S} / \mathrm{H}]$ and a $20 \mathrm{kHz}$ square wave was applied to the $\mathrm{S} / \mathrm{H}$ 's clock input. Therefore, the $960 \mathrm{~Hz}$ sinusoid was sampled 21 times.

\begin{tabular}{|c|c|c|c|}
\hline Parameter & $\mathrm{TT}$ process, $+27^{\circ} \mathrm{C}$ & $\mathrm{FF}$ process, $-40^{\circ} \mathrm{C}$ & $\mathrm{SS}$ process, $+125^{\circ} \mathrm{C}$ \\
\hline SNR $(\mathrm{dB})$ & 80.1 & 78.1 & 81.0 \\
\hline $\operatorname{SINAD}(\mathrm{dB})$ & 77.5 & 76.0 & 78.1 \\
\hline ENOB (bit) & 12.6 & 12.3 & 12.7 \\
\hline THD (dB) & -80.9 & -80.1 & -81.3 \\
\hline SFDR $(\mathrm{dB})$ & 84.7 & 84.8 & 84.8 \\
\hline
\end{tabular}

Table 5.3: Simulated $\mathrm{S} / \mathrm{H}$ performance for $\mathrm{TT}$, FF, and $\mathrm{SS}$ process and $+27^{\circ} \mathrm{C},-40^{\circ} \mathrm{C}$, and $+125^{\circ} \mathrm{C}$ temperatures. The $\mathrm{S} / \mathrm{H} /$ performance parameters are $\mathrm{SNR}$, SINAD, ENOB, THD, and SFDR and were measured in Matlab using the frequency spectrums shown in Figure 5.11, Figure 5.12, and Figure 5.13 respectively. These process and temperature process points are commonly known as the "Industrial Corners".

\subsection{SAR Logic and Capacitor Array Verification}

The SAR logic and capacitor array were functionally verified via a transient analysis performed using the Cadence Spectre simulator. An analog input, clock, and reset were 
applied to the SAR logic. The SAR has two sets of outputs. One set controls switches in the capacitor array to control the charge on specific capacitors to generate the required voltage. The second set of outputs is a serial clock and data interface. This interface is the output of the Charge Sharing SAR ADC.

The simulation results when $1 \mathrm{~V}$ is applied to the input of the SAR is shown in Figure 5.14. Figure 5.14 is an example of the SAR and capacitor array verification. Many such simulations were performed to verify the SAR and capacitor array.

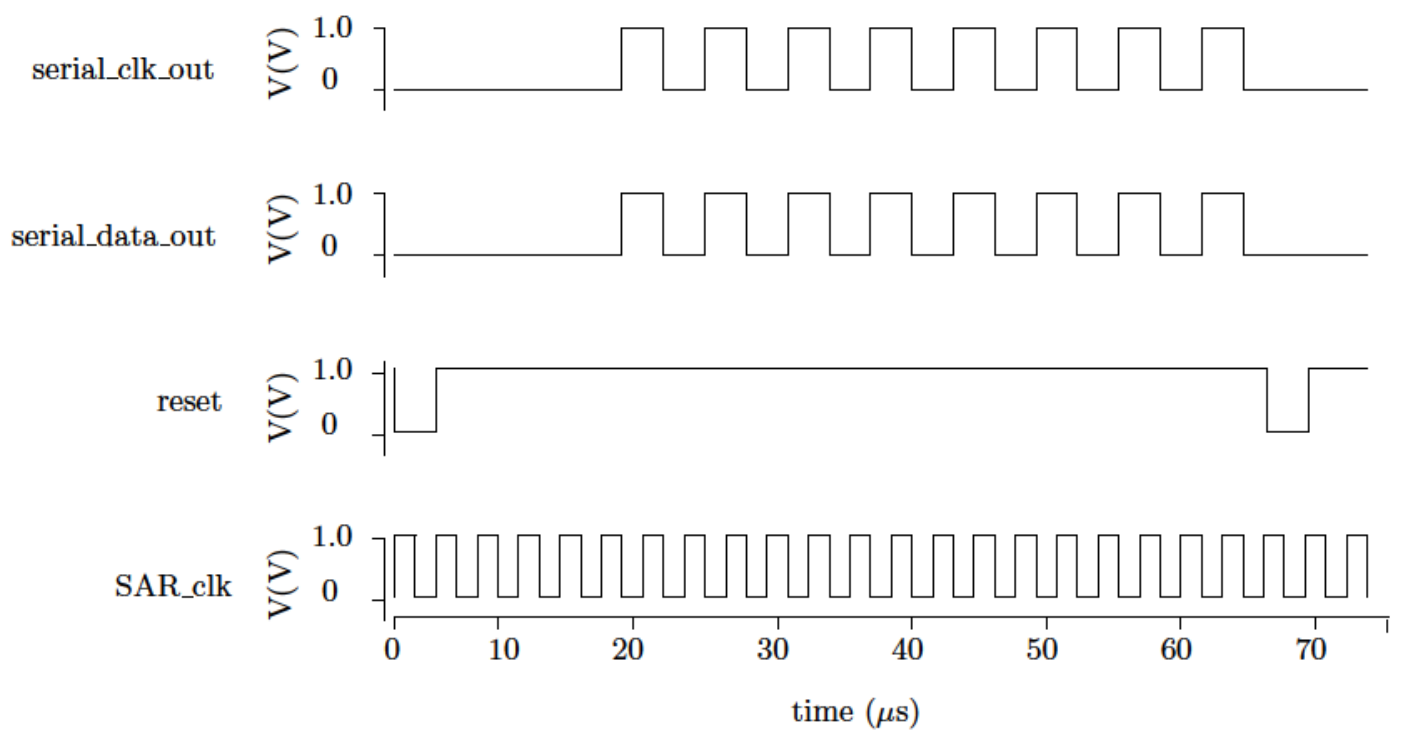

Figure 5.14: SAR/capacitor array transient analysis. The figure shows the serial interface's resulting waveforms in response to $1 \mathrm{~V}$ applied to the SAR $\mathrm{s}$ input. 


\subsection{Full Charge Sharing SAR ADC Verification}

\subsubsection{Full Charge Sharing SAR ADC SNR, SINAD, ENOB, THD, and SFDR}

Part of the full charge sharing [SAR ADC verification consisted of measuring the ADC's SNR, SINAD, ENOB, THD, and SFDR at three process points, typical nfet/pfet and $+27^{\circ} \mathrm{C}$, slow nfet $/$ pfet and $-40^{\circ} \mathrm{C}$, fast nfet $/$ pfet and $+125^{\circ} \mathrm{C}$. The measurements were performed using a method similar to the $\mathrm{S} / \mathrm{H}$. A transient analysis was performed and the resulting full $\mathrm{ADC}$ s output and applied analog input were written out of Cadence and then read into Matlab. The Matlab code performed a[FFT] and then calculated SNR, SINAD, ENOB, THD, and SFDR.

The $\mathrm{ADC}$ s performance in the TT process point at $27^{\circ} \mathrm{C}$ is shown in Figure 5.15 .

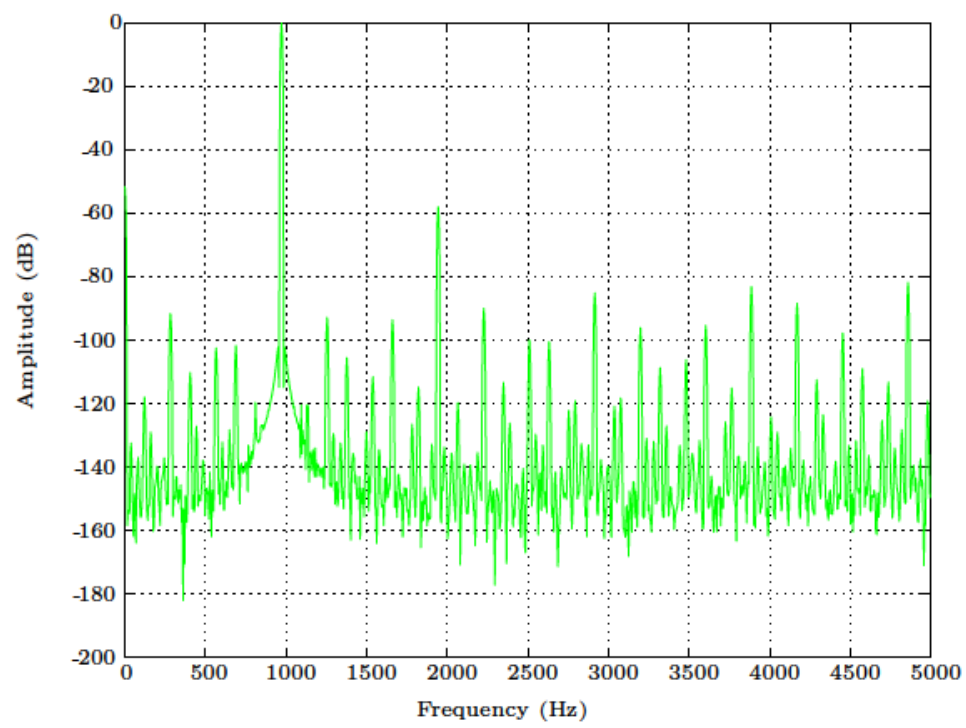

Figure 5.15: Full ADC frequency spectrum generated using Matlab. This spectrum was used to measure $\mathrm{ADC}$ s performance for TT process and $+27^{\circ} \mathrm{C}$. The measured $\mathrm{SNR}=49.7 \mathrm{~dB}, \mathrm{SINAD}=48.72$ $\mathrm{dB}, \overline{\mathrm{ENOB}}=7.8 \mathrm{bits}, \mathrm{THD}=-60.3 \mathrm{~dB}$, and $\mathrm{SFDR}=60.4 \mathrm{~dB}$. A $960 \mathrm{~Hz}$ sine wave was applied to the input of the $\mathrm{S} / \mathrm{H}$ and the frequency of the SAR clock was $300 \mathrm{kHz}$. 
The $\mathrm{ADC}$ s performance using the $\mathrm{FF}$ corner at $-40^{\circ} \mathrm{C}$ is shown in Figure 5.16 .

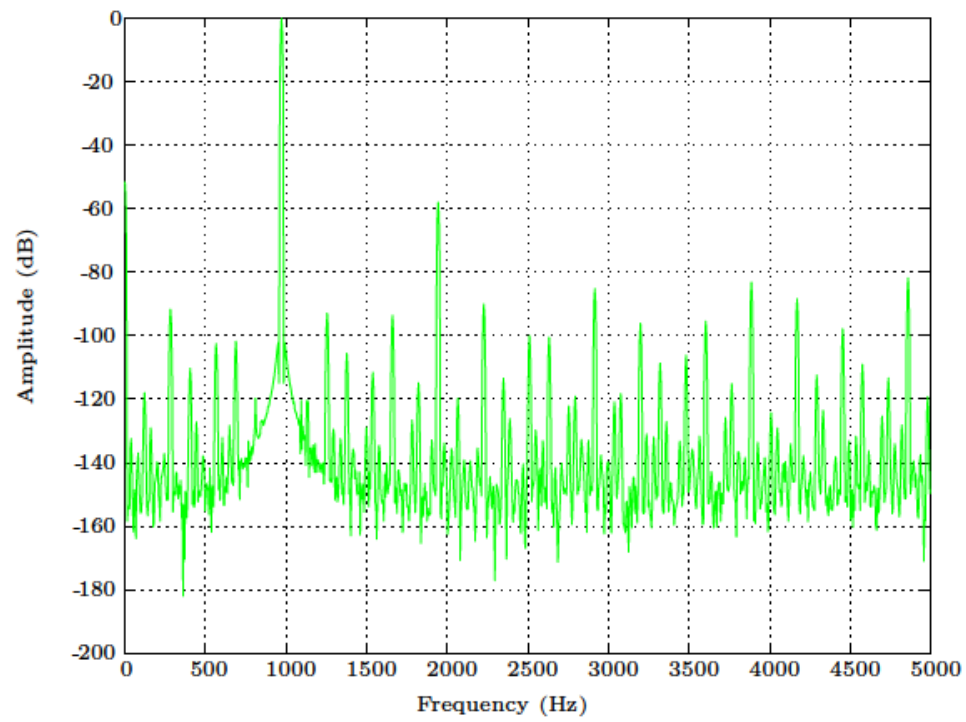

Figure 5.16: Full ADC frequency spectrum generated using Matlab. This spectrum was used to measure ADC]s performance for $\left[\mathrm{FF}\right.$ process and $-40^{\circ} \mathrm{C}$. The measured $\mathrm{SNR}=50.2 \mathrm{~dB}, \mathrm{SINAD}=49.44 \mathrm{~dB}$, $\mathrm{ENOB}=7.92 \mathrm{bits}, \mathrm{THD}=-61.5 \mathrm{~dB}$, and $\mathrm{SFDR}=61.8 \mathrm{~dB}$. A $960 \mathrm{~Hz}$ sine wave was applied to the input of the $[\mathrm{S} / \mathrm{H}]$ and the frequency of the SAR clock was $300 \mathrm{kHz}$.

The $\mathrm{ADC}$ s performance using the $\mathrm{SS}$ corner at $+125^{\circ} \mathrm{C}$ is shown in Figure 5.17.

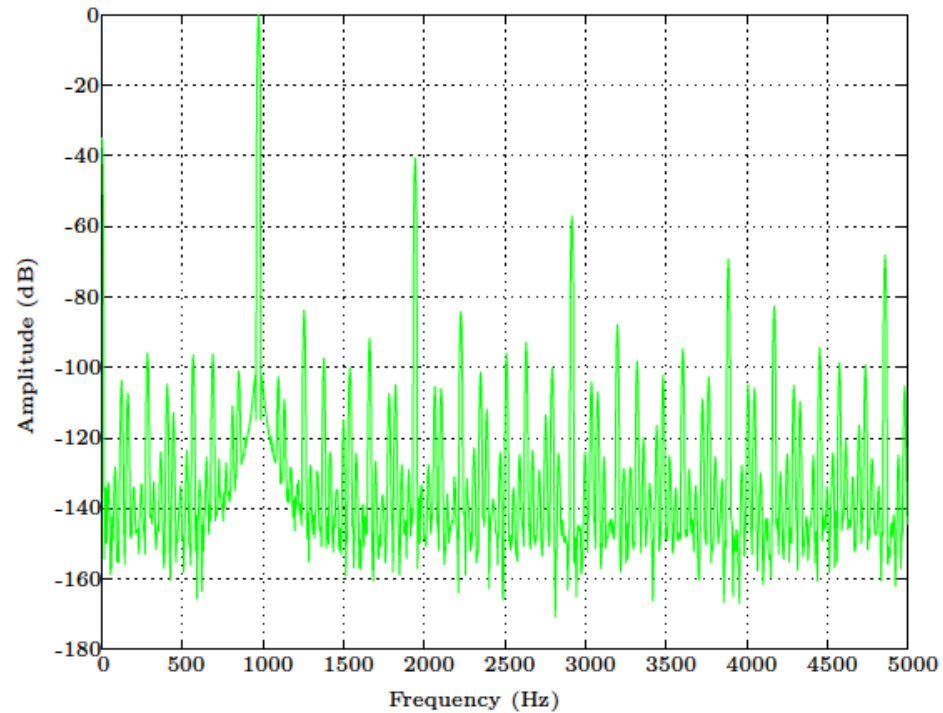

Figure 5.17: Full ADC frequency spectrum generated using Matlab. This spectrum was used to measure $\mathrm{ADC}$ /s performance for $\mathrm{SS}$ process and $+125^{\circ} \mathrm{C}$. The measured $\mathrm{SNR}=35.2 \mathrm{~dB}, \mathrm{SINAD}=34.6$ $\mathrm{dB}, \quad \mathrm{ENOB}=5.4 \mathrm{bits}, \mathrm{THD}=-42.9 \mathrm{~dB}$, and $\mathrm{SFDR}=43 \mathrm{~dB}$. A $960 \mathrm{~Hz}$ sine wave was applied to the input of the $[\mathrm{S} / \mathrm{H}$ and the frequency of the SAR clock was $300 \mathrm{kHz}$. 


\begin{tabular}{|c|c|c|c|}
\hline Parameter & $\mathrm{TT}$ process, $+27^{\circ} \mathrm{C}$ & $\mathrm{FF}$ process, $-40^{\circ} \mathrm{C}$ & $\mathrm{SS}$ process, $+125^{\circ} \mathrm{C}$ \\
\hline SNR $(\mathrm{dB})$ & 49.9 & 50.2 & 35.2 \\
\hline $\operatorname{SINAD}(\mathrm{dB})$ & 48.72 & 49.44 & 34.6 \\
\hline ENOB (bit) & 7.8 & 7.92 & 5.4 \\
\hline THD (dB) & -60.3 & -61.5 & -42.9 \\
\hline $\operatorname{SFDR}(\mathrm{dB})$ & 60.4 & 61.8 & 43 \\
\hline
\end{tabular}

Table 5.4: Simulated ADC performance for TT, FF, and $\mathrm{SS}$ process and $+27^{\circ} \mathrm{C},-40^{\circ} \mathrm{C}$, and $+125^{\circ} \mathrm{C}$ temperatures. The ADC performance parameters are SNR, SINAD, ENOB, THD, and SFDR, and were measured in Matlab using the frequency spectrums shown in Figure 5.15, Figure 5.16, and Figure 5.17 respectively. These process and temperature process points are commonly known as the "Industrial Corners".

\subsubsection{Full Charge Sharing SAR ADC DNL and INL}

The next part of the full charge sharing SAR ADC verification consisted of measuring the ADCls DND and INL.

The output of an ideal ADC is divided into $2^{\mathrm{N}}$ uniform steps. Each step has the same width. Deviation from this ideal step width is calledDNL. INL is the maximum deviation of the $\mathrm{ADC}$ s output voltage from its ideal value. A missing code will occur when either $\mathrm{DNL}$ or INL is greater than LSB/2.

The histogram method was used to perform DNL and INL measurements as described in [27].

The histogram method requires the application of a triangular wave to the ADC?s input and recording the ADC's digital output. The number of recorded samples is denoted as $\mathrm{M}_{\mathrm{T}} \cdot \mathrm{M}_{\mathrm{T}}$ is calculated using Equation 5.7.

$$
M_{\mathrm{T}}=2^{\mathrm{N}}(S)
$$

where, $M_{T}$ is the number of recorded samples, $N$ is the number of $A D C$ bits $(N=8$ for this $\mathrm{ADC}$, and $\mathrm{S}$ is the desired number of hits per bin. The variable $\mathrm{S}$ describes the required number of occurrences in a digital code bin. 
The charge sharing SAR ADC has 8-bits and it is desired that the number of occurrences in a digital code bin is 14 . Therefore, using Equation 5.7, $\mathrm{M}_{\mathrm{T}}$ is calculated to be 3584 .

The triangular wave is required to have a slow ramp. The time for the ramp to make a full scale transition is the ADCls sampling period multiplied by $\mathrm{M}_{\mathrm{T}}$, which is $179.2 \mathrm{~ms}$.

$\mathrm{DNL}$ is calculated by substituting Equation 5.8 into Equation 5.9 . The DNL in terms of an LSB that was measured during simulation is shown in Figure 5.18 .

$$
h(n)_{\text {theoretical }}=\frac{M_{\mathrm{T}}}{2^{\mathrm{N}}-2}
$$

where, $\mathrm{M}_{\mathrm{T}}$ and $\mathrm{N}$ were previously defined terms used by Equation 5.7 .

$$
D N L(n)=\left(\frac{h(n)_{\text {actual }}}{h(n)_{\text {theoretical }}}\right)-1
$$

where, $\mathrm{h}(\mathrm{n})_{\text {actual }}$ is the number of occurrences in each code bin.

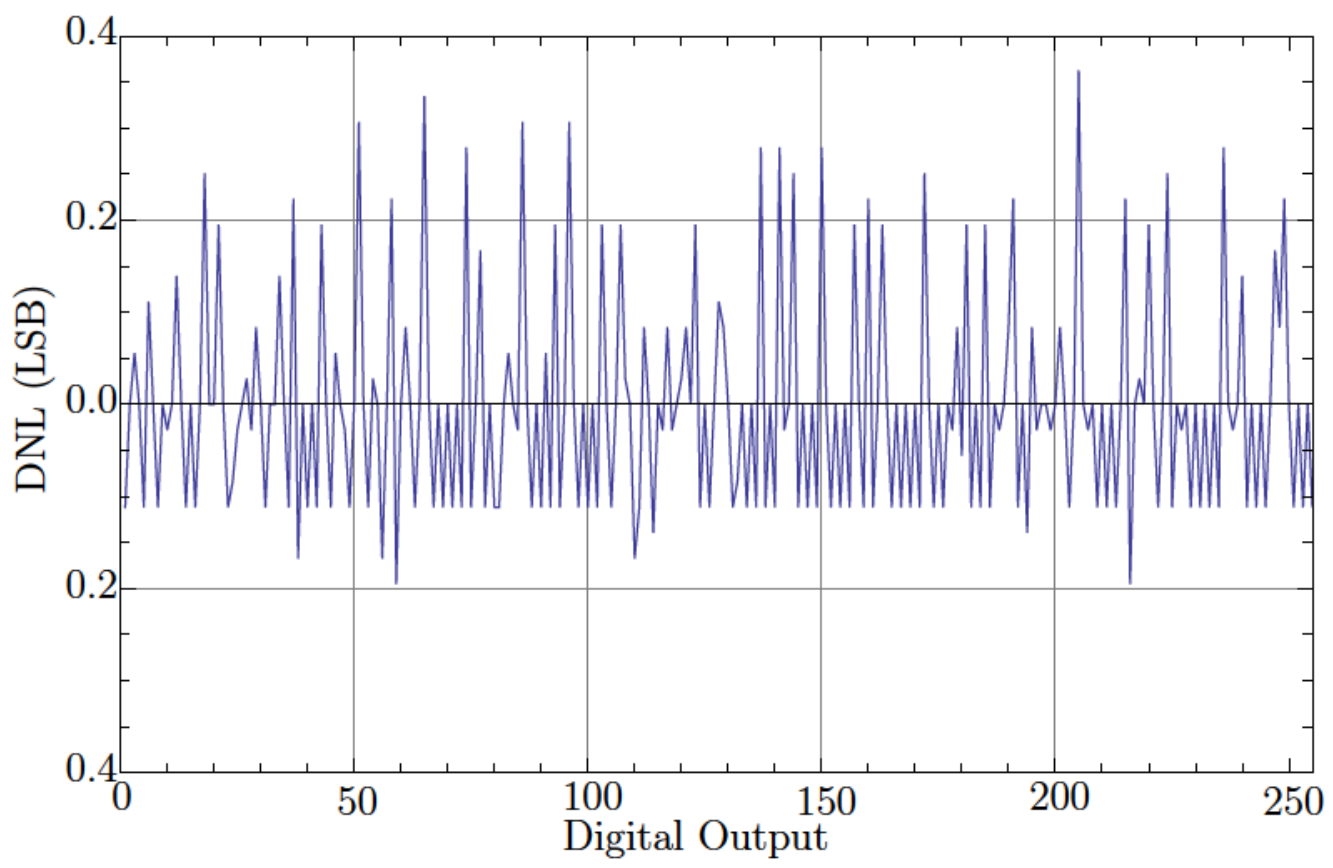

Figure 5.18: Simulated ADC DNL. The worst case DNL was 0.3627LSB.

INL is calculated by integrating DNL. INL was calculated using Equation 5.10. The 
INL in terms of an LSB that was measured during simulation is shown in Figure 5.19 .

$$
I N L(n)=\sum_{i=1}^{n} D N L_{i}
$$

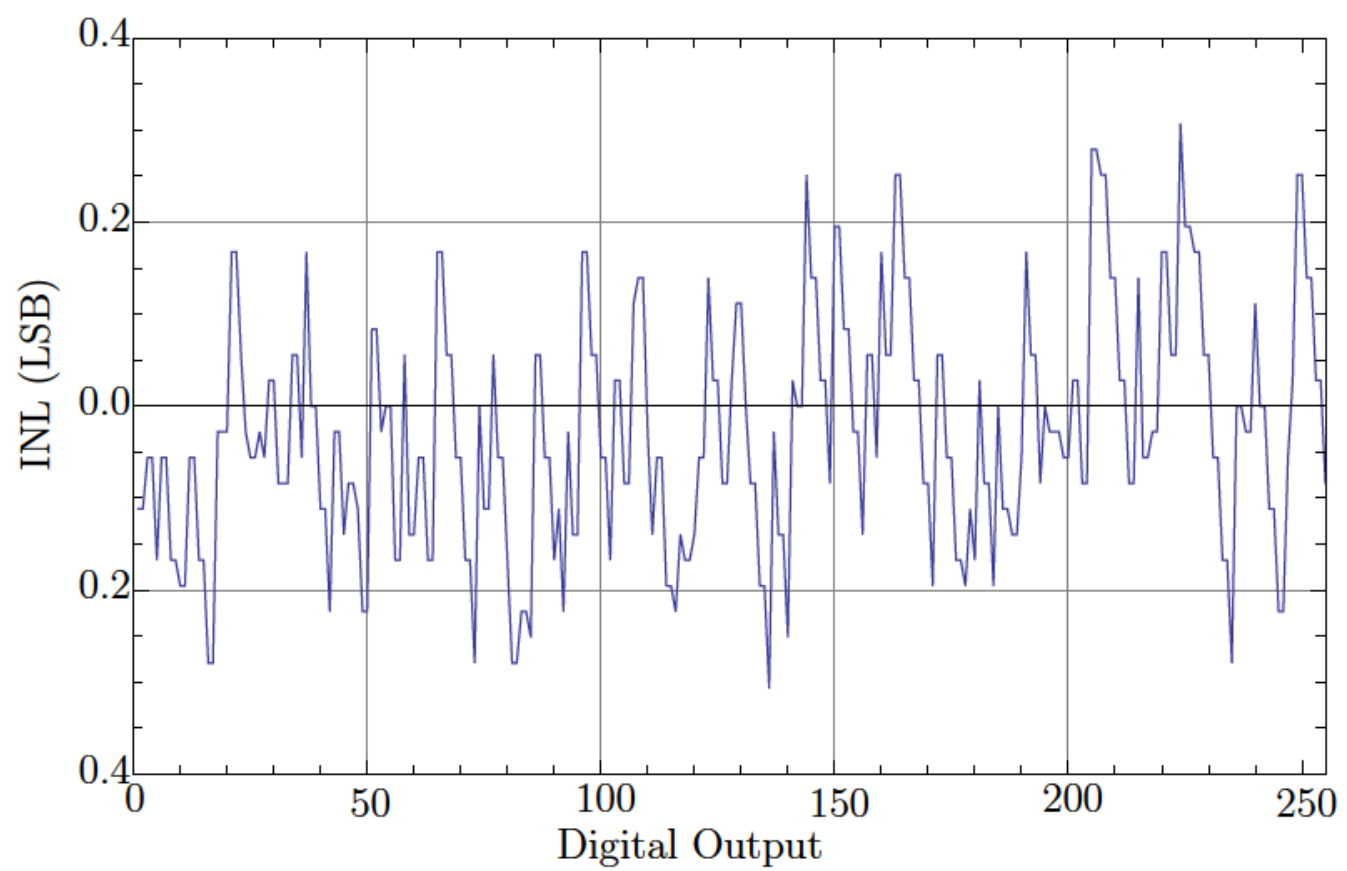

Figure 5.19: Simulated ADC INL The wost case INL was 0.3069LSB. 


\subsection{Leakage Current Simulations}

Chapter 6 shows that the measured performance of the fabricated charge sharing SAR ADC doesn't match the simulations shown in this chapter. The measured lab results show the voltage stored across the capacitors leak at a very fast rate.

It was found that previous versions of the IBM CMRF8SF library didn't model leakage [28]. To determine if the transistor leakage is modeled in the version of the IBM CMRF8SF library used to develop this work, a single transistor was simulated and its drain and substrate currents were measured.

\subsubsection{IBM CMRF8SF FET Drain and Body Leakage Current}

The circuit shown in Figure 5.20 was simulated using a pfet transistor from the IBM CMRF8SF library (CMCL Revision: 1.11 10/02/22 10:22:46). The drain and substrate currents are plotted in Figure 5.21. The voltage $\mathrm{V}_{\mathrm{sb}}$ is a one volt supply that is only used to measure substrate current.

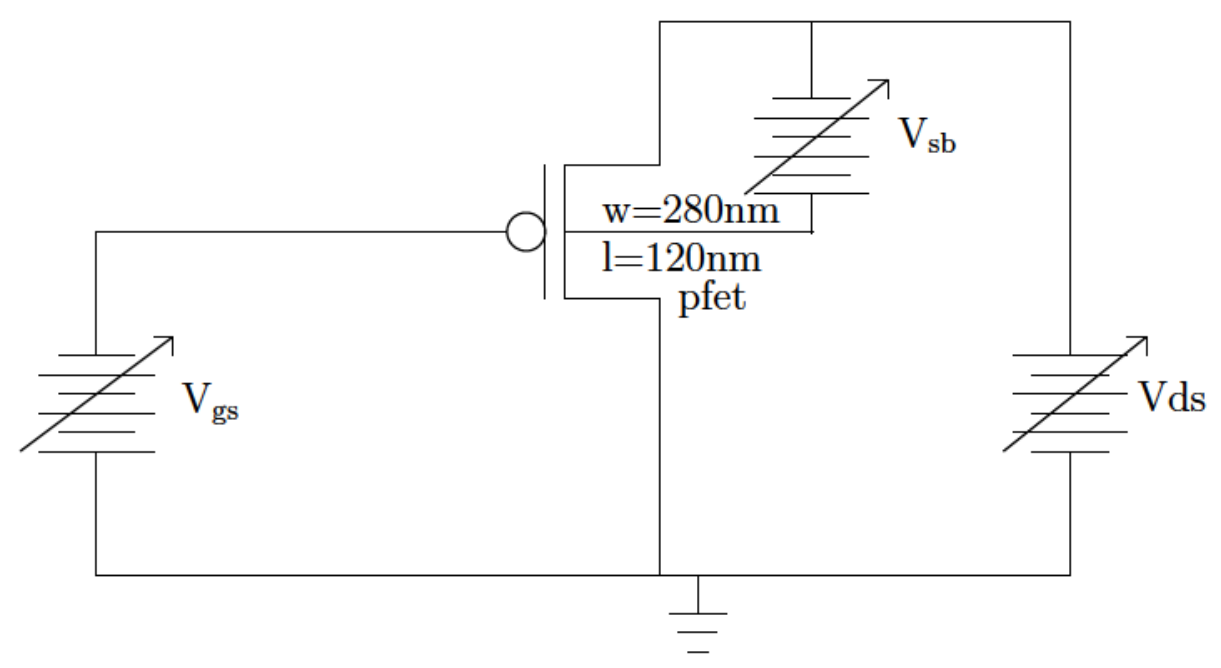

Figure 5.20: CMRF8SF pfet transistor leakage test circuit.

The drain and substrate currents shown in Figure 5.21 were generated by sweeping $\mathrm{V}_{\mathrm{gs}}$ in Figure 5.20. It is expected that as $\mathrm{V}_{\mathrm{gs}}$ is increased from $-1 \mathrm{~V}$ to a voltage less than $0 \mathrm{~V}$, that both the drain and substrate currents will decrease. These currents should decrease due to an effect known as GIDL. As $V_{\mathrm{gs}}$ is increased further, the substrate current will increase due junction leakage currents. Then as $\mathrm{V}_{\mathrm{gs}}$ is further increased, 


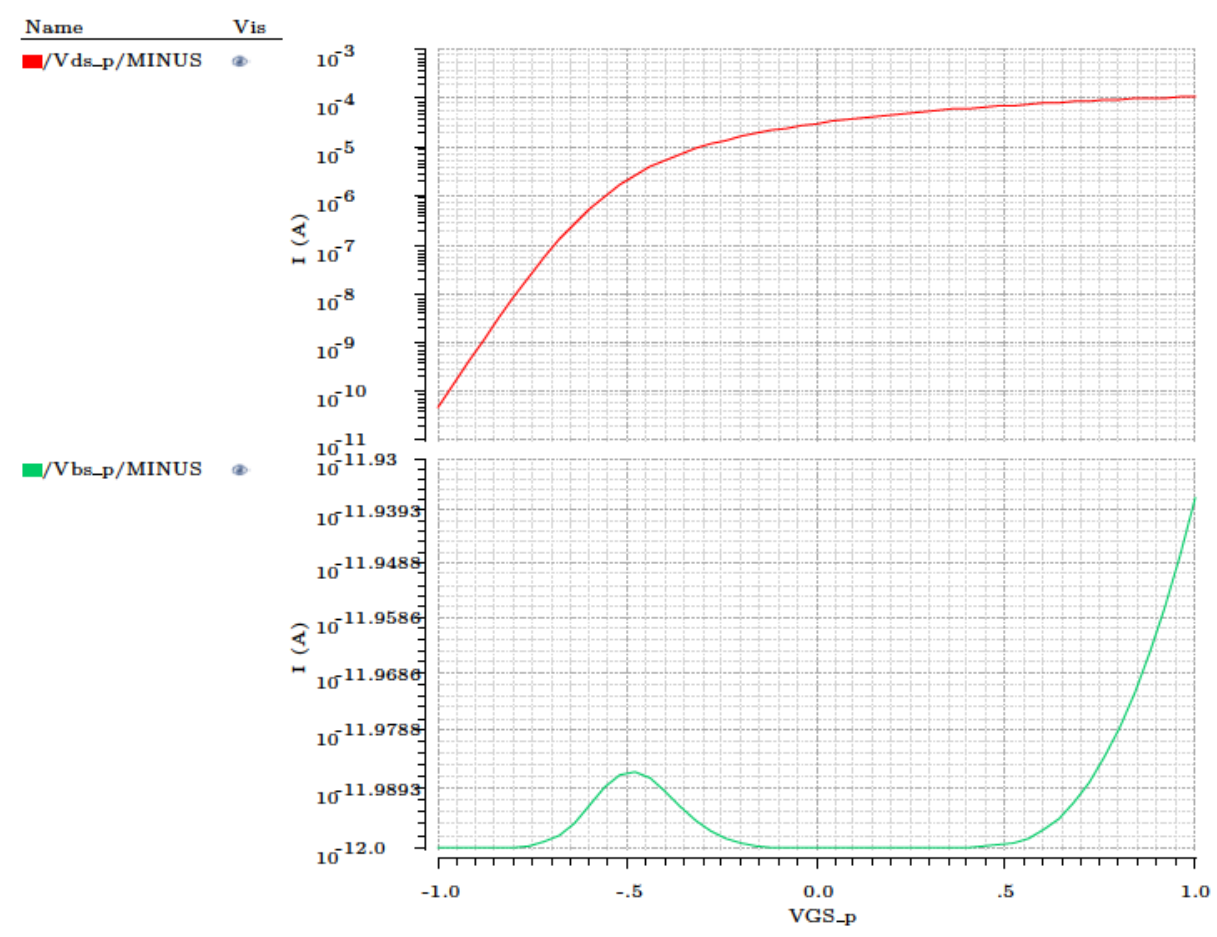

Figure 5.21: CMRF8SF pfet transistor source-body and drain-source currents.

substrate currents increase due to impact ionization. All of these effects are evident in Figure 5.21 so, therefore, it is concluded that the IBM CMRF8SF library models leakage currents.

GIDL is described in [29] as a current that is generated at the drain junction when operating in accumulation. As $\mathrm{V}_{\mathrm{gs}}$ is made more negative, direct gate tunneling is the dominant current in GIDL, which is independent of $\mathrm{V}_{\mathrm{ds}}$.

The junction leakage currents are currents that flow through the reverse-bias pn junctions that exist in the drain to well and source to well junctions. These pn leakage currents have two main components. The first component is minority carrier diffusion that exists near the edge of the depletion region. The second component is due to electron-hold pair generation in the depletion region of the reversed-bias junction [30].

Impact ionization occurs when electrons strike silicon atoms and create electron/hole pairs. As the electric field increases, for example due to increasing $\mathrm{V}_{\mathrm{gs}}$, the electron's energy increases and more electron/hole pairs are created.

The circuit shown in Figure 5.22 was simulated using an nfet transistor from the IBM CMRF8SF library (CMCL Revision: 1.11 10/02/22 10:22:46). The drain and substrate currents are plotted in Figure 5.23. The power supply, $\mathrm{V}_{\mathrm{sb}}$ is a $0 \mathrm{~V}$ substrate current 
measuring point.

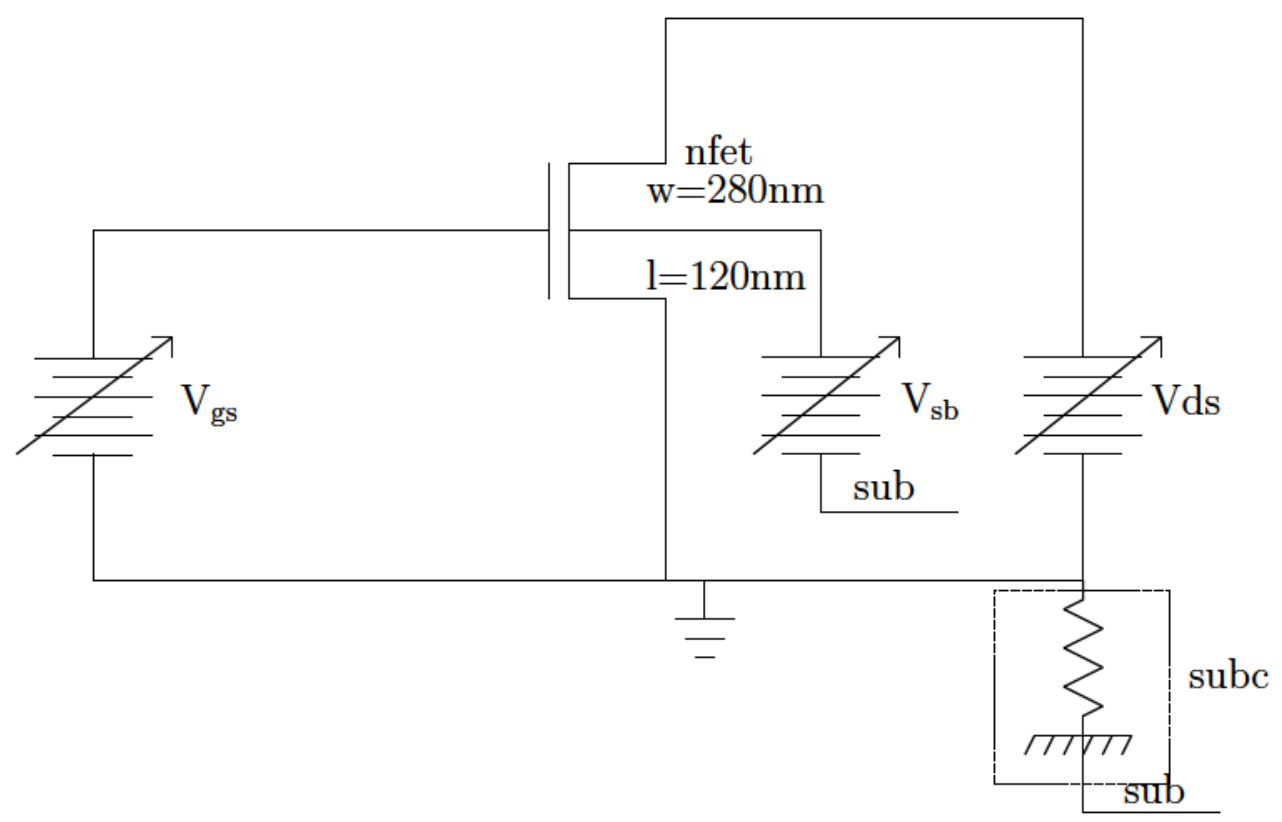

Figure 5.22: CMRF8SF pfet transistor leakage test circuit.

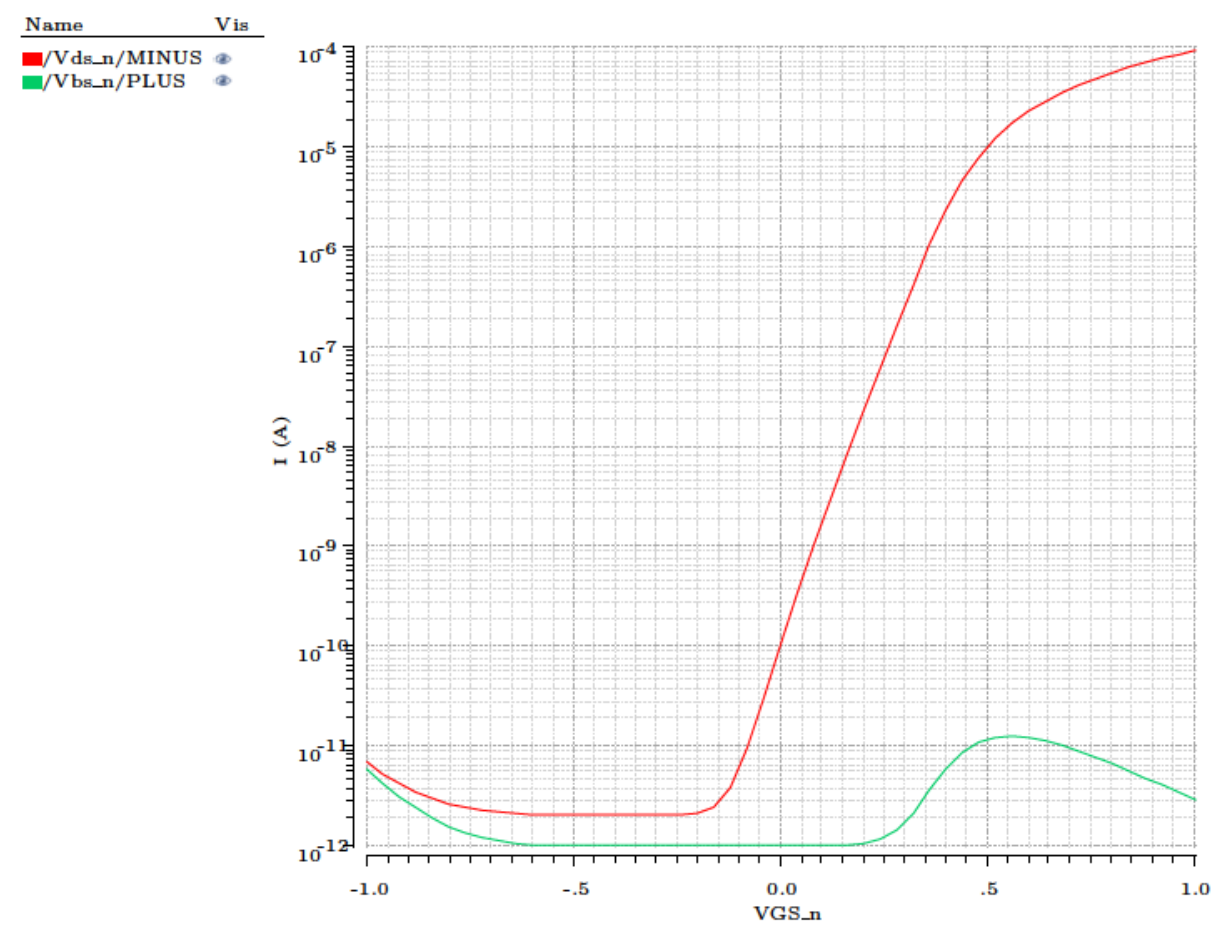

Figure 5.23: CMRF8SF nfet transistor source-body and drain-source currents.

Again, as was the case of the pfet, the GIDL, junction leakage currents, and impact ionization are evident in the simulated substrate current. Therefore, leakage effects are modeled. 


\section{Chapter 6}

\section{Charge Sharing SAR ADC Bench Evaluation}

\subsection{Introduction}

This chapter describes the measured results of packaged parts. Since these results didn't match the simulation results, it was decided to measure unpackaged die to determine if the process of packaging die caused ESD damage. The die was then inspected using microscopes and SEM to determine if the issues were due to manufacturing faults. Finally, the results of [1]'s work are compared to the results of this work.

\subsection{Packaged Die Measurements}

The lab bench evaluation consisted of the packaged die connected to the test equipment shown in Figure 6.1 .

The analog signal and clock applied to the packaged die were supplied by the Agilent 33210A function generators. The function generators were configured so that the signals they output had a worst case undershoot of $0 \mathrm{~V}$ and worst case overshoot of $1.09 \mathrm{~V}$. The current source was a Keithly 6220 current source. A Keithly 2602 was used to measure the output of the Keithly 6220 . Analog $(1.2 \mathrm{~V})$ and digital $(1 \mathrm{~V})$ was provided by the HP E3610A power supplies. The supplies were configured so that when their output was 


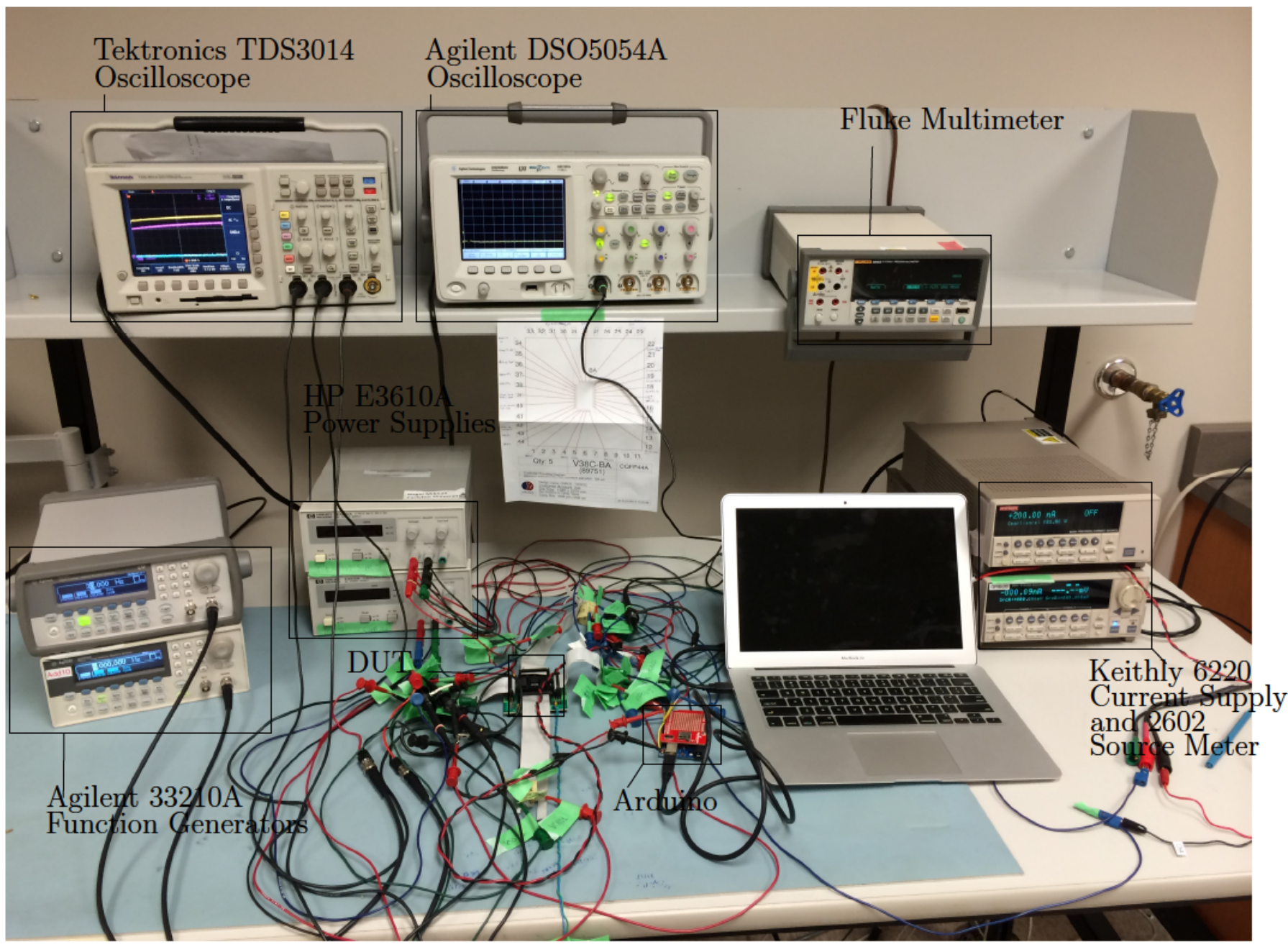

Figure 6.1: Packaged die lab bench evaluation setup. The analog signal and clock were supplied by the Agilent 33210 A function generators. The current source was a Keithly 6220 current source. A keithly 2602 was used to measure the source current. Analog (1.2V) and digital (1V) was provided by the HP E3610A power supplies. Signals were measured using Tektronics TPS3014 and Agilent DSO5054A Oscilloscopes. An Arduino and laptop were used to capture the ADC's output.

short circuited, the maximum current supplied was $50 \mathrm{~mA}$. Signals were measured using Tektronics TPS3014 and Agilent DSO5054A Oscilloscopes via 10x probes. An Arduino and laptop were used to capture the $\mathrm{ADC}$ s output. The Arduino's serial peripheral interface (SPI) interface operated at $4 \mathrm{MHz}$ and was used to capture the serial clock and data from the ADC. The data captured by the Arduino was analyzed in Matlab.

To evaluate packaged die, they were placed in a 44 pin clam-shell socket mounted on a simple printed circuit board $(\mathrm{PCB})$. This socket and $\mathrm{PCB}$ are available from the $\mathrm{CMC}$ (CMC part number CFP44CSTF) and are shown in Figure 6.2. 


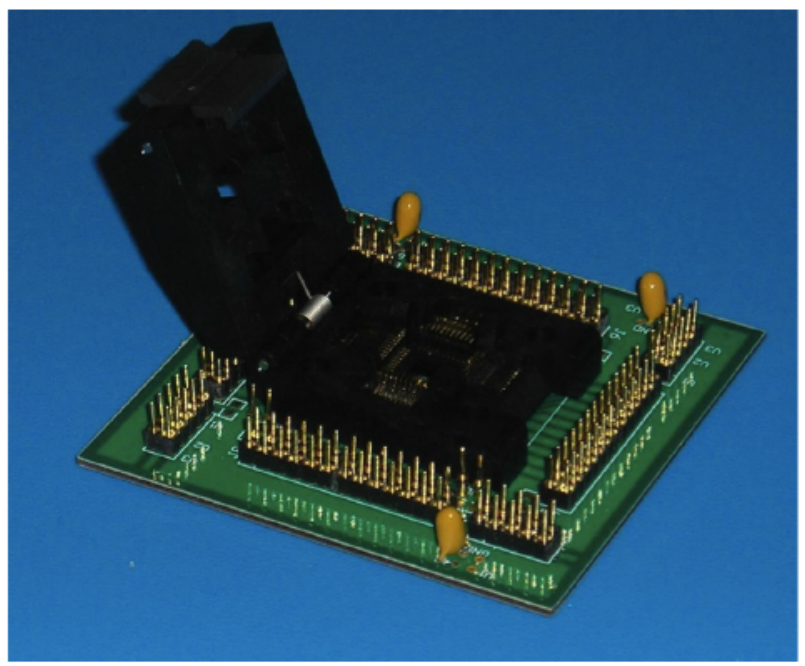

Figure 6.2: The 44 pin clamshell socket and breakout board available from CMC.

Unfortunately, as Figure 6.3 shows, the $\overline{\mathrm{S} / \mathrm{H}}$ 's capacitor discharged when the $\mathrm{S} / \mathrm{H}$ entered hold mode.

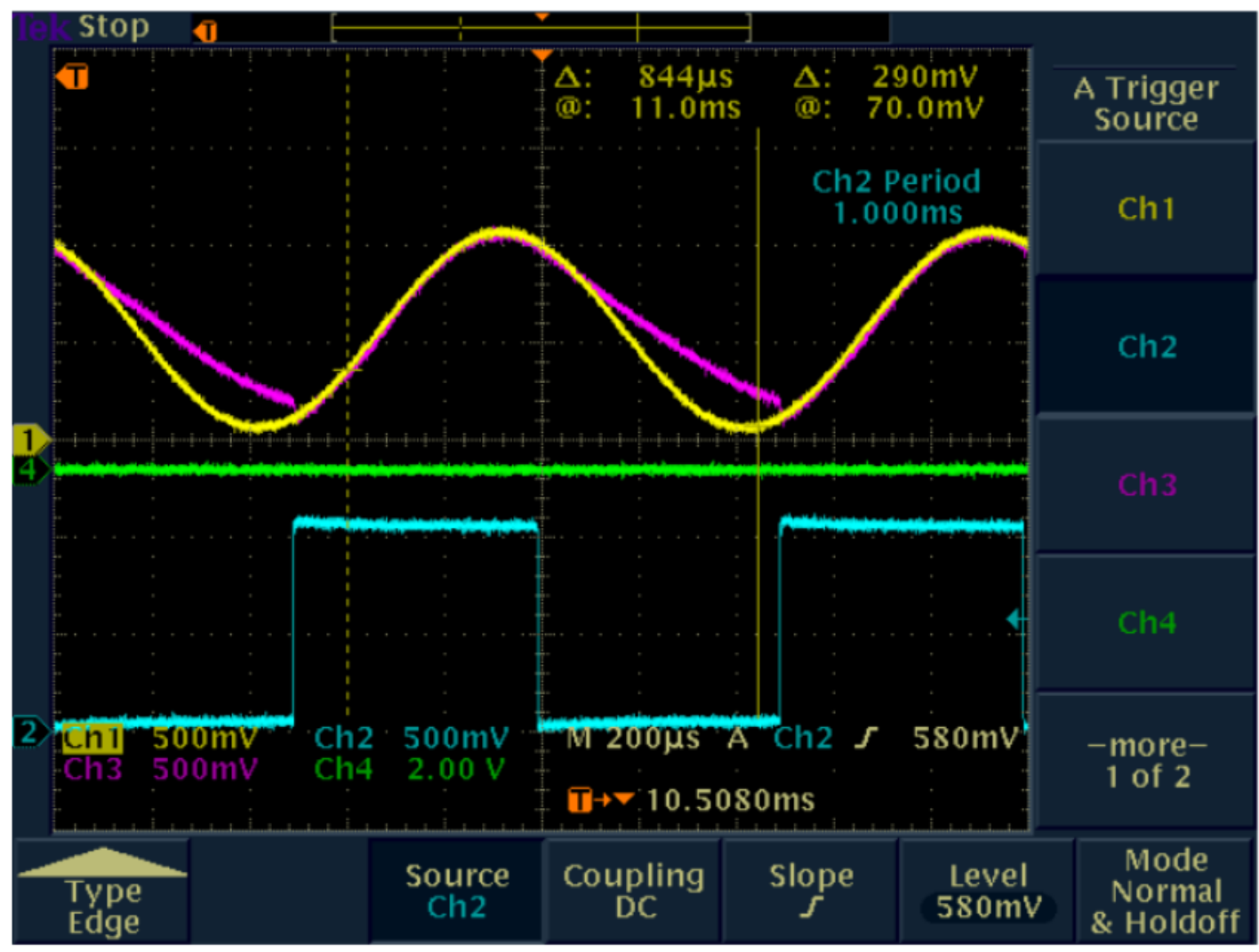

TDS $3014-6: 26: 41$ PM 12/18/2013

Figure 6.3: Measured $1 \mathrm{kHz}$ analog input (channel 1), $1 \mathrm{kHz} \mathrm{S} / \mathrm{H}$ clock (channel 2), $\mathrm{S} / \mathrm{H}$ output (channel 3). $[\mathrm{S} / \mathrm{H}$ output follows analog input when clock is high - track mode. But the $\mathrm{S} / \mathrm{H}$ capacitor discharges when clock is low - hold mode. 


\subsection{Bare Die Probing Measurements}

Unpackaged die were probed in order to rule out the possibility that ADC die were damaged during packaging. Figure 6.4 shows the probing station used to probe the unpackaged die.

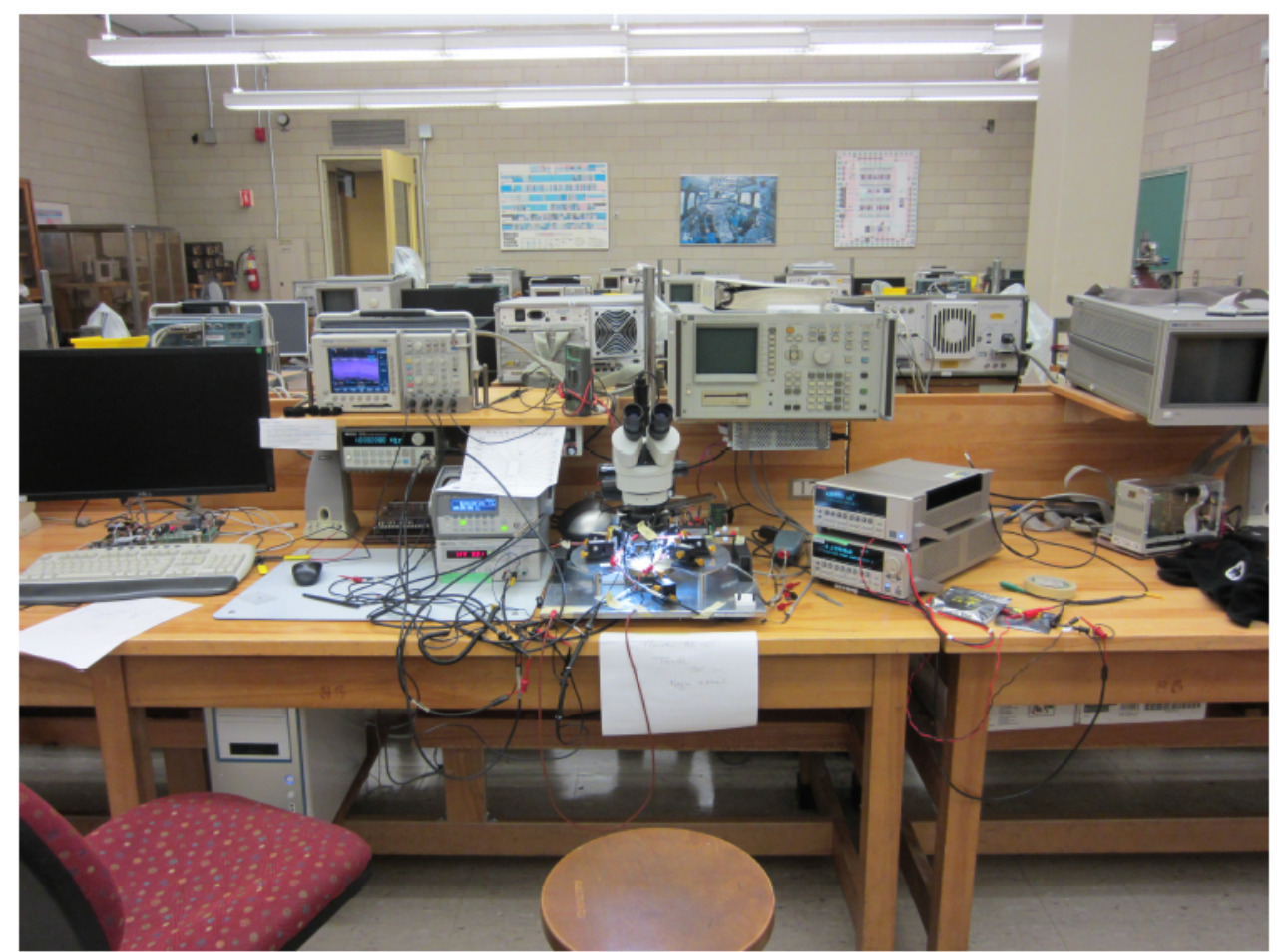

Figure 6.4: Lab bench configuration used to probe unpackaged die. Also shown are the function generators that provide the analog and clock inputs, power supply, Oscilloscope, current supply and current meter, and probing station.

Figure 6.5 shows a close up view of the die mounted on a conductive wafer while being probed.

Figure 6.6 shows the same conductive wafer being probed but this time, the view is from above the wafer. 


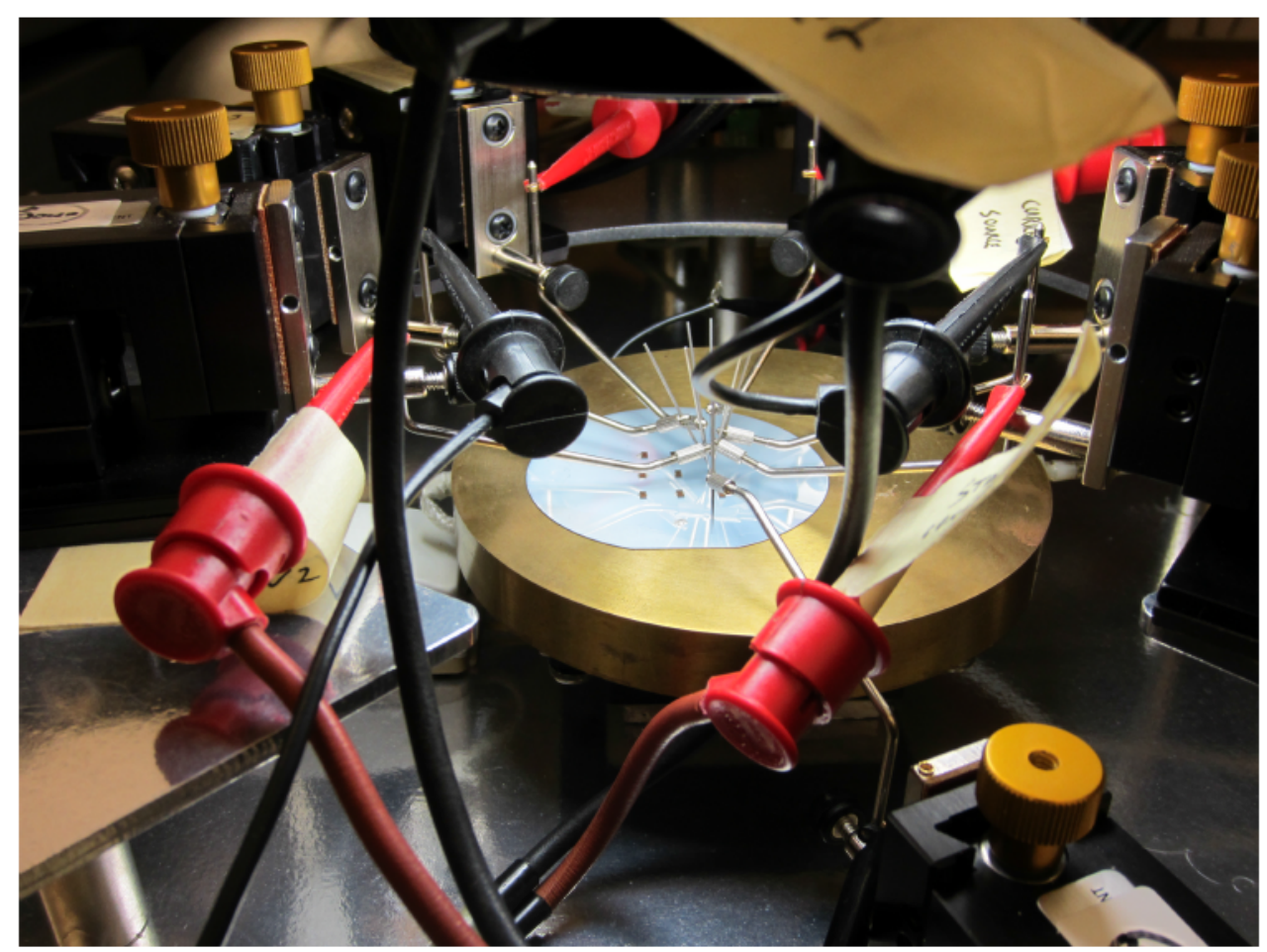

Figure 6.5: Nine die mounted on conductive plate where one die is probed using six needles. The needles supply the analog input, $\mathrm{S} / \mathrm{H} / \mathrm{s}$ clock, power, ground, current supply, and measure the $\mathrm{S} / \mathrm{H} / \mathrm{s}$ output.

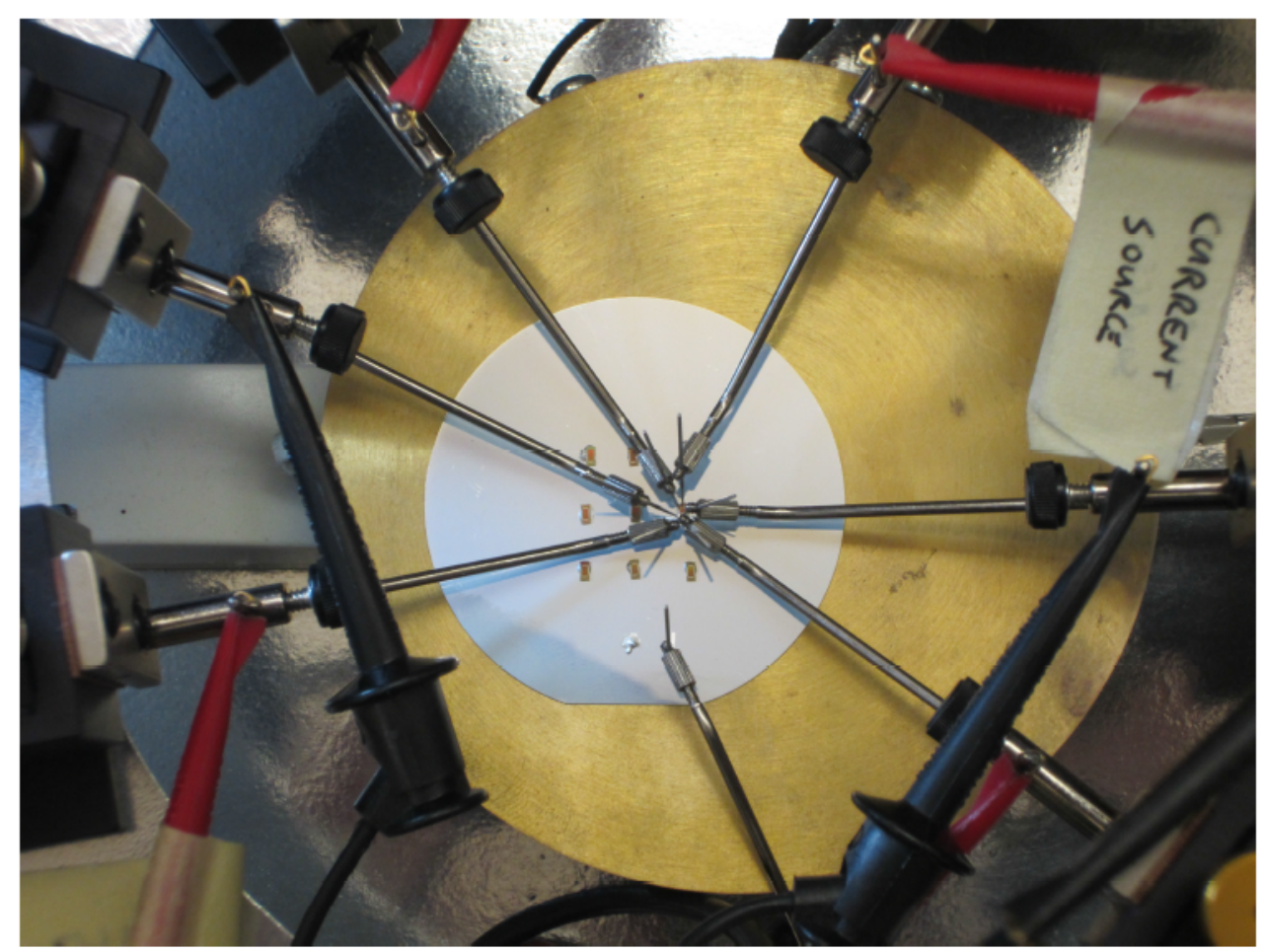

Figure 6.6: Nine die mounted on conductive plate where one die is probed using six needles. View is looking down from above. 
Several experiments were performed where unpackaged die were mounted to a conductive plate as shown in Figure 6.7, mounted on a glass slide, and the previously shown die mounted on a conductive wafer Figure 6.5 .

The experiment using the conductive plate as shown in Figure 6.7 was abandoned due to the fact that the plate had a slight warp and didn't adhere to the probing station. The plate had a tendency to move when probed and all die were damaged as they were probed.

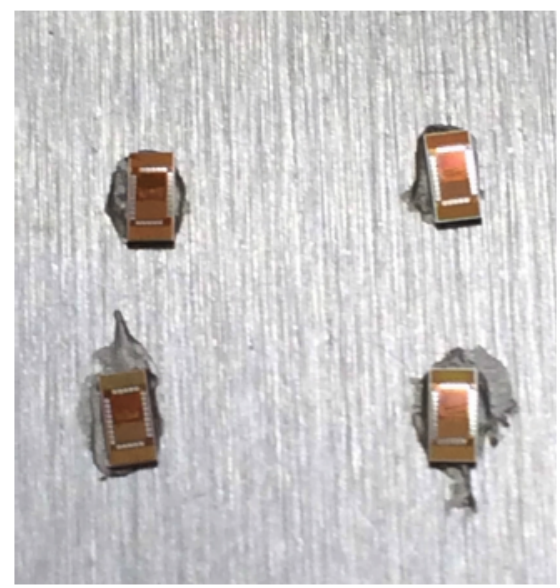

Figure 6.7: Four die mounted on conductive plate. This experiment was thrown out because the plate wasn't flat and moved too easily.

The experiments using the conductive wafer as shown in Figure 6.5 and glass plate showed the same capacitor discharge behavior as was observed with the packaged parts (shown in Figure 6.3). Therefore, it was ruled out that the packaging had damaged the die. The reason for mounting die on the glass plate and on a conductive plate was to check if grounding of the die's substrate (by grounding the conductive plate) made a difference in the die's behavior. It was suspected that the substrate connection to ground could be the reason for the S/H's capacitor discharge. It was found that grounding the substrate made no difference in the $\mathrm{S} / \mathrm{H}$ 's behavior.

While the unpackaged die were being mounted on the conductive wafer and metal plate, it was observed that the die had a strange bubbling. This bubbling is known as "chicken pox". A five hundred times optical zoom of the die is shown in Figure 6.8. As Figure 6.8 shows, the "chicken pox" are numerous. 


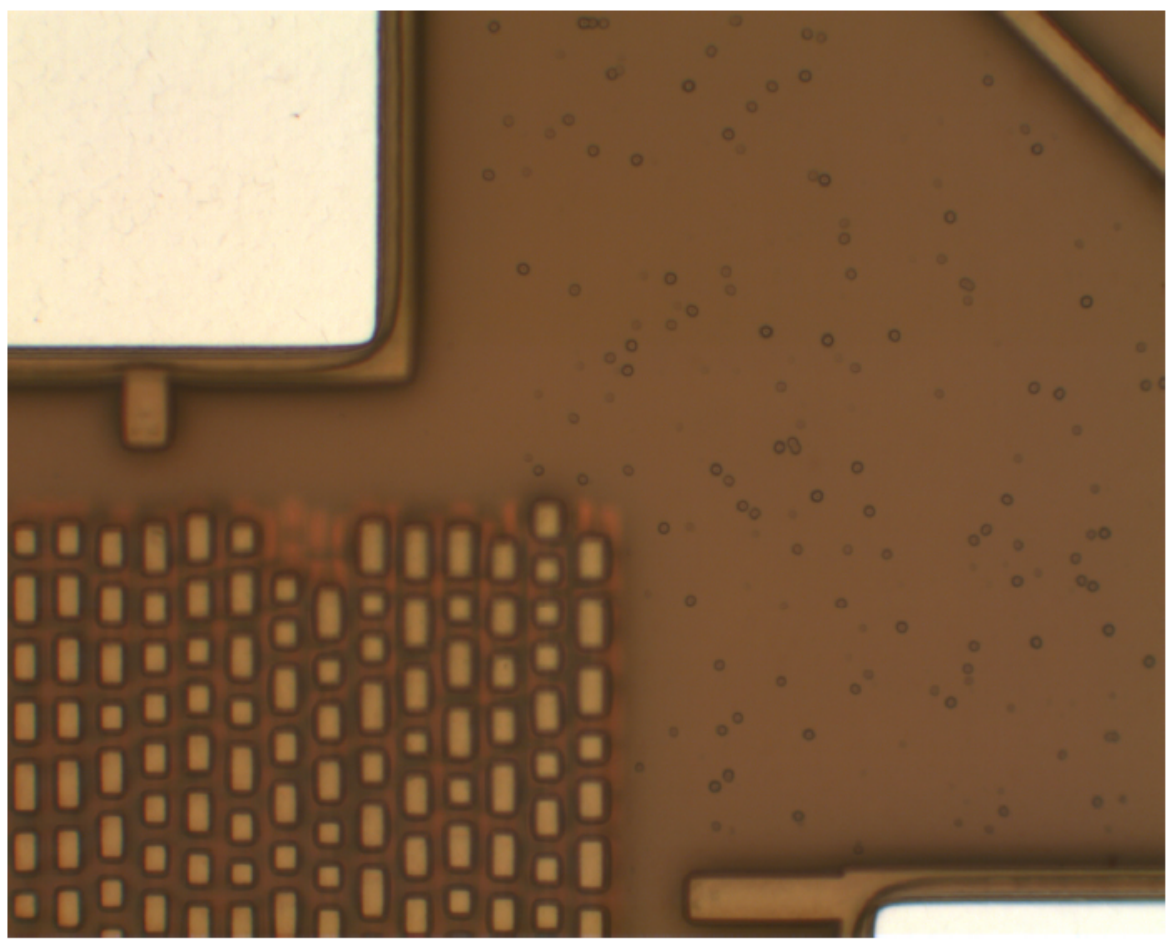

Figure 6.8: Five hundred times zoom in of die to show "chicken pox".

Figure 6.9 shows a one thousand times zoom of the unpackaged die to show the "chicken pox" more closely. As Figure 6.9 shows that on average, the "chicken pox" are $1.95 \mu \mathrm{m}$ in diameter.

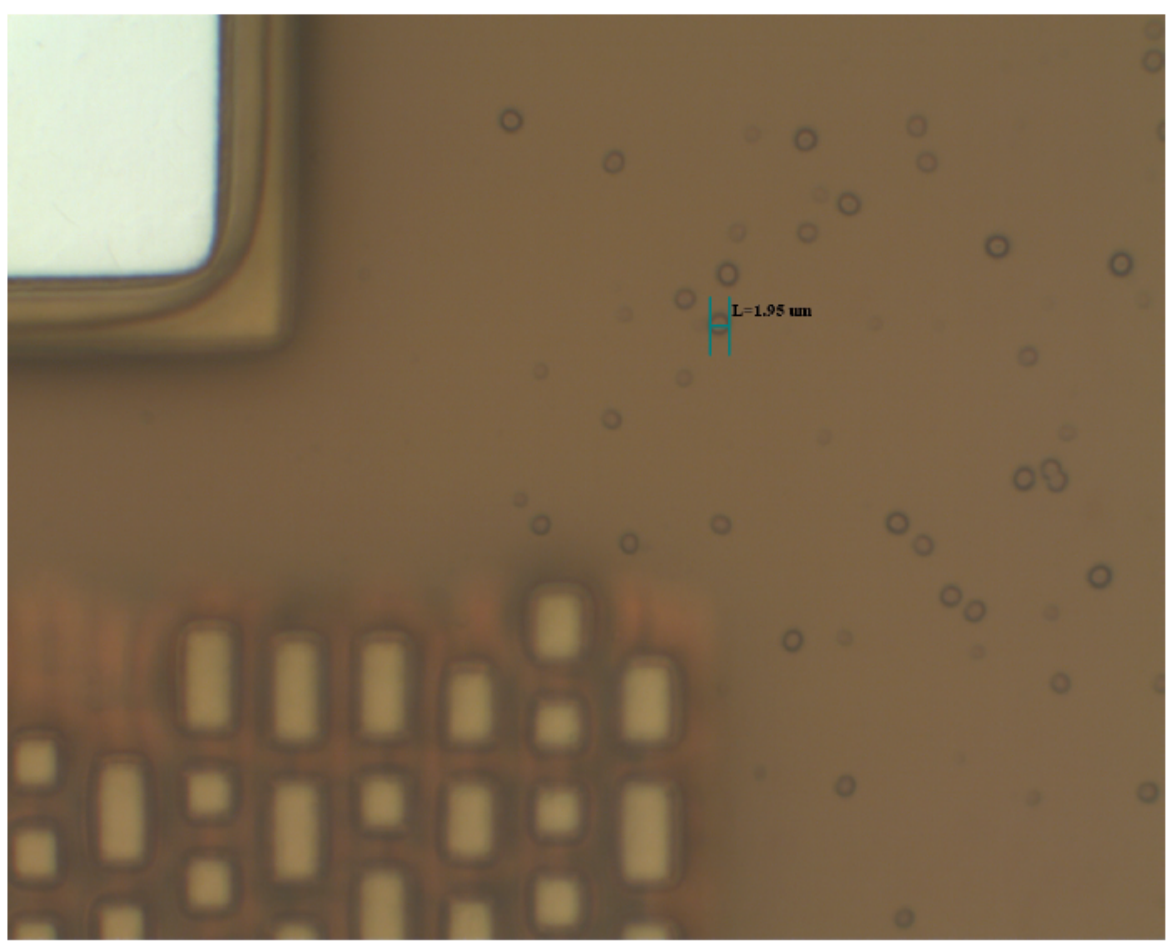

Figure 6.9: One thousand times zoom in of die to show "chicken pox" more clearly. The average sized bubble is measured to show their approximate size. 
Figure 6.10 shows an example of a full unpackaged die. As Figure 6.10 shows, the "chicken pox" are visible over the entire die.

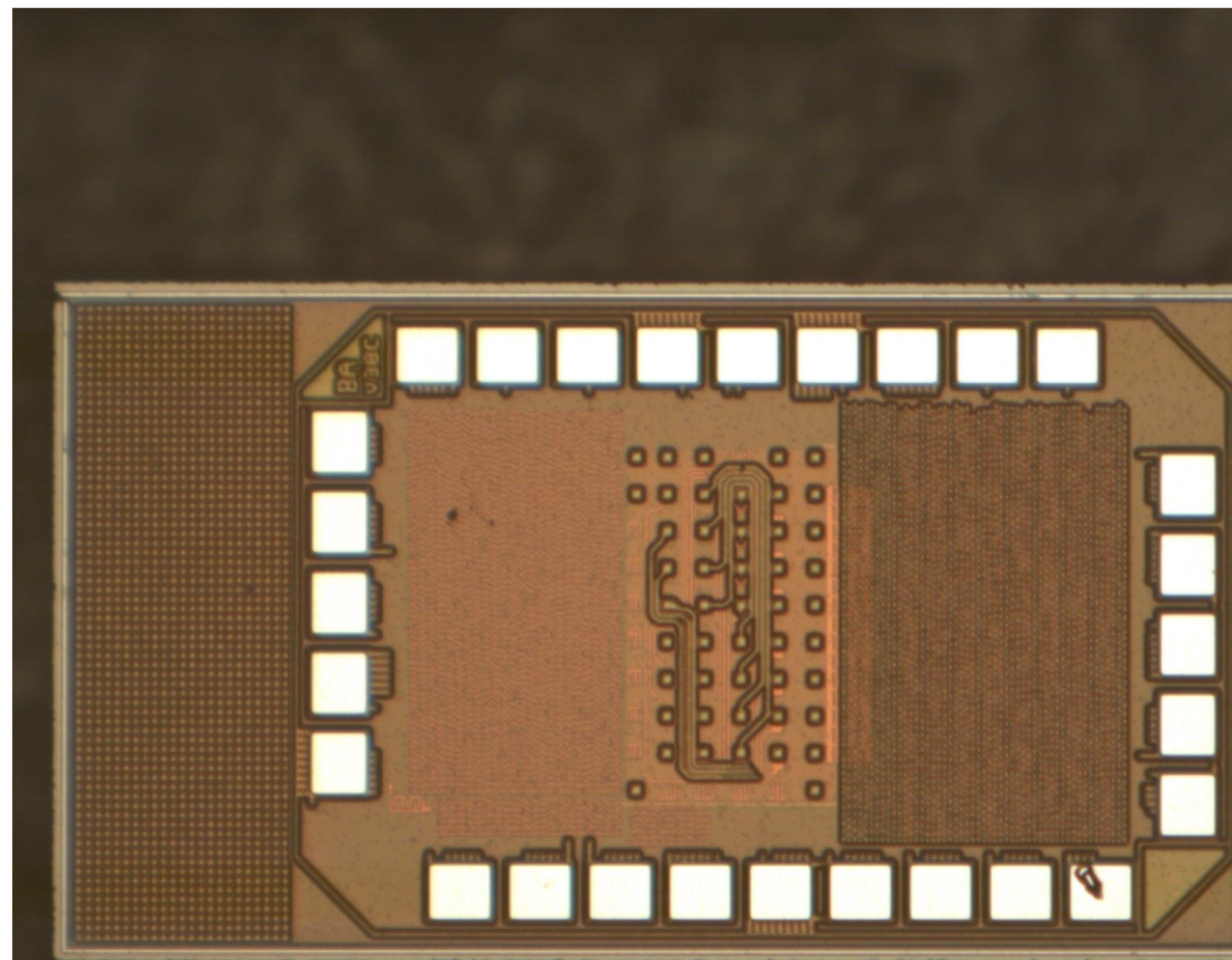

Figure 6.10: Full view of die to show "chicken pox" are present over entire die. This was typical of die received.

In order to determine where the "chicken pox" are located, an unpackaged die was analyzed using a SEM. Figure 6.11 shows that the "chicken pox" are below the surface of the die.

A die was then cleaved in order to perform cross sectional analysis of the die. 

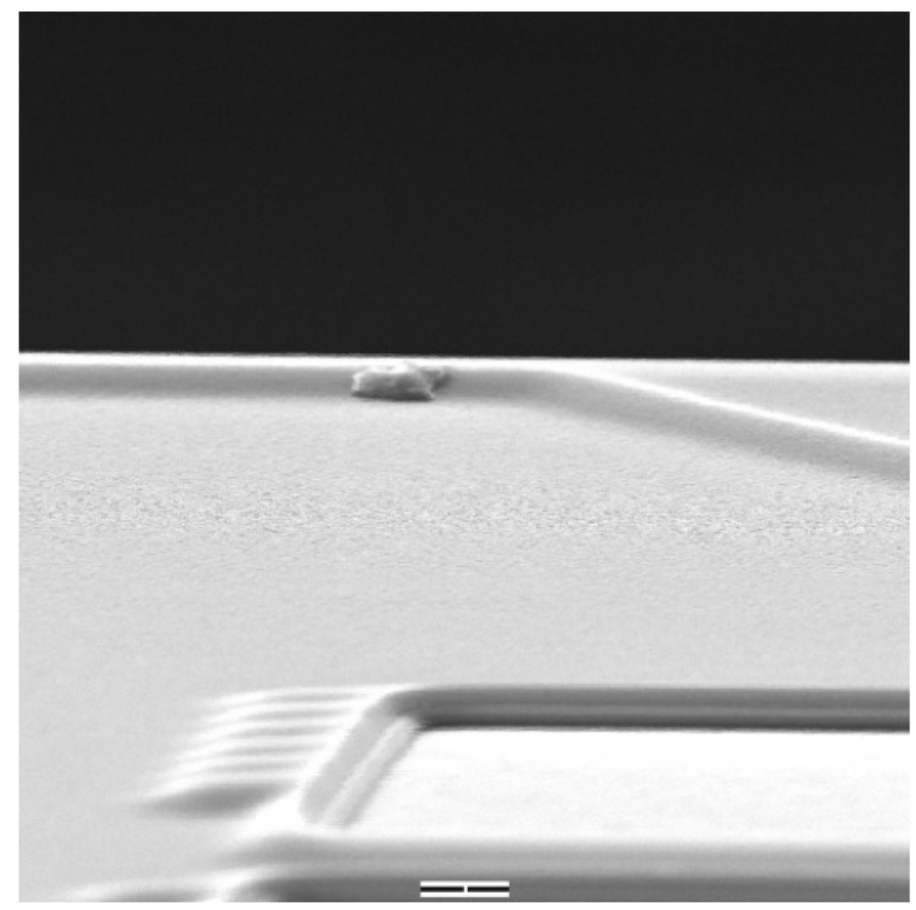

Figure 6.11: Surface of die captured using SEM. This shows that the "chicken pox" are not at the surface but at some layer within the layer stackup. A deposit of something is visible in the figure but this isn't the "chicken pox".

Figure 6.12 shows a SEM image of a "chicken pox" bubble at a location deep within the cleaved die.

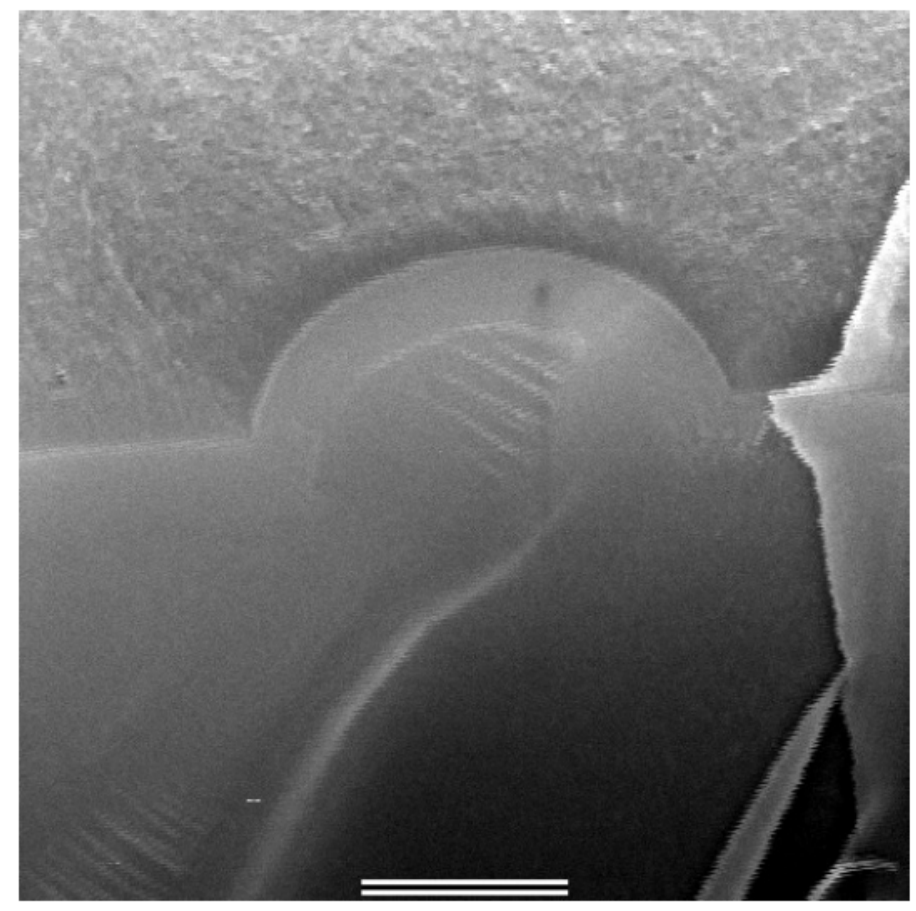

Figure 6.12: SEM picture showing "chicken pox" bubble. Note that this bubble is approximately $2 \mu \mathrm{m}$ in diameter as expected.

Figure 6.13 shows a SEM image of a cracked VIA in the cleaved die. It was unclear 
if the cracked VIA was due to the cleaving process or was a manufacturing defect. So a clean cleave was performed at Fibics to determine if the VIA was cracked during the cleaving process.

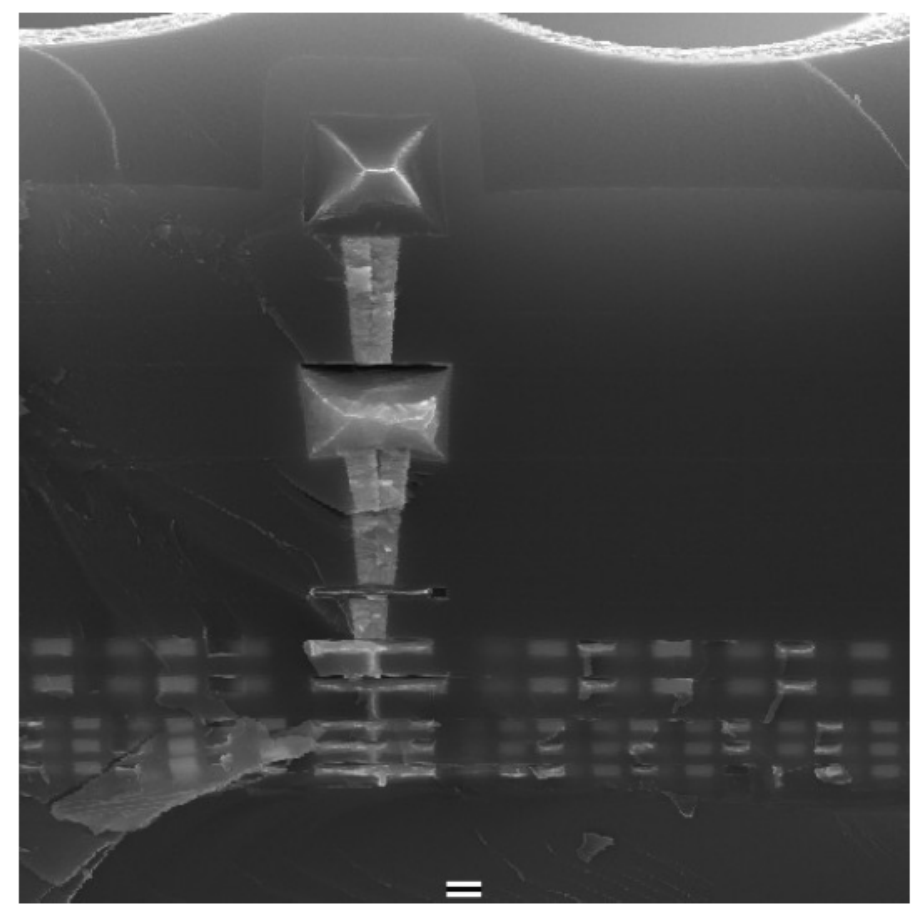

Figure 6.13: $[$ SEM picture of VIA stack. It is unclear if the VIA was cracked as a result of cleaving. 


\subsection{Fibics SEM}

A further analysis of the unpackaged die was performed at Fibics. The die was cleaved and a Gallium beam was used to remove die material. The Fibics focused ion beam $(\mathrm{FIB})$ /scanning electron microscope (SEM) station is shown in Figure 6.14.

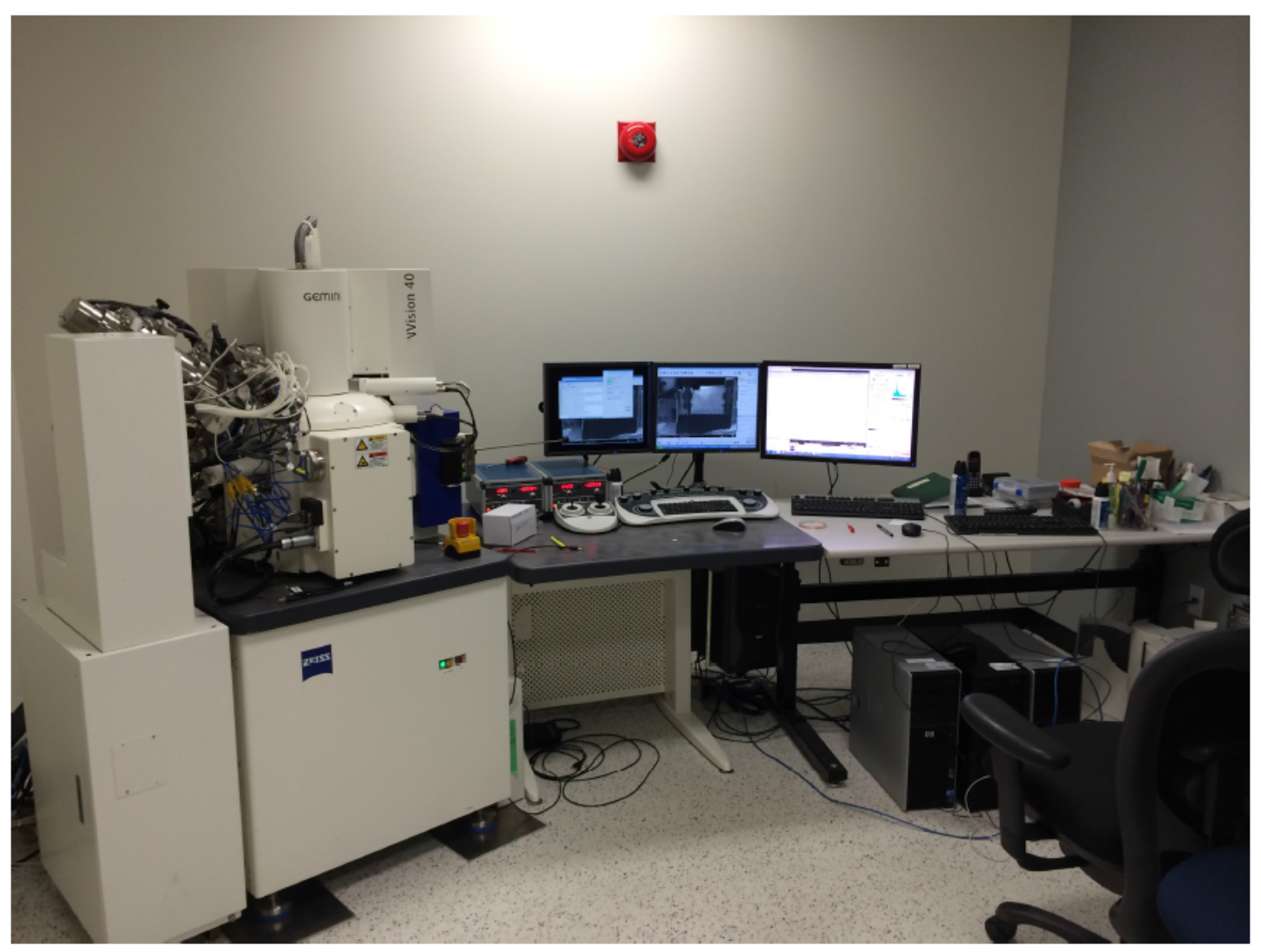

Figure 6.14: Fibics Incorporated FIB SEM station.

Many hours of removing die material using the Gallium beam produced the image of the "chicken pox" bubbles shown in Figure 6.15. No VIAs were found during these many hours of analysis. Analysis time at Fibics is very expensive so it was not possible to perform an exhaustive scan to find the VIAs. The VIA that was found to be cracked in Figure 6.13 was found by chance. 


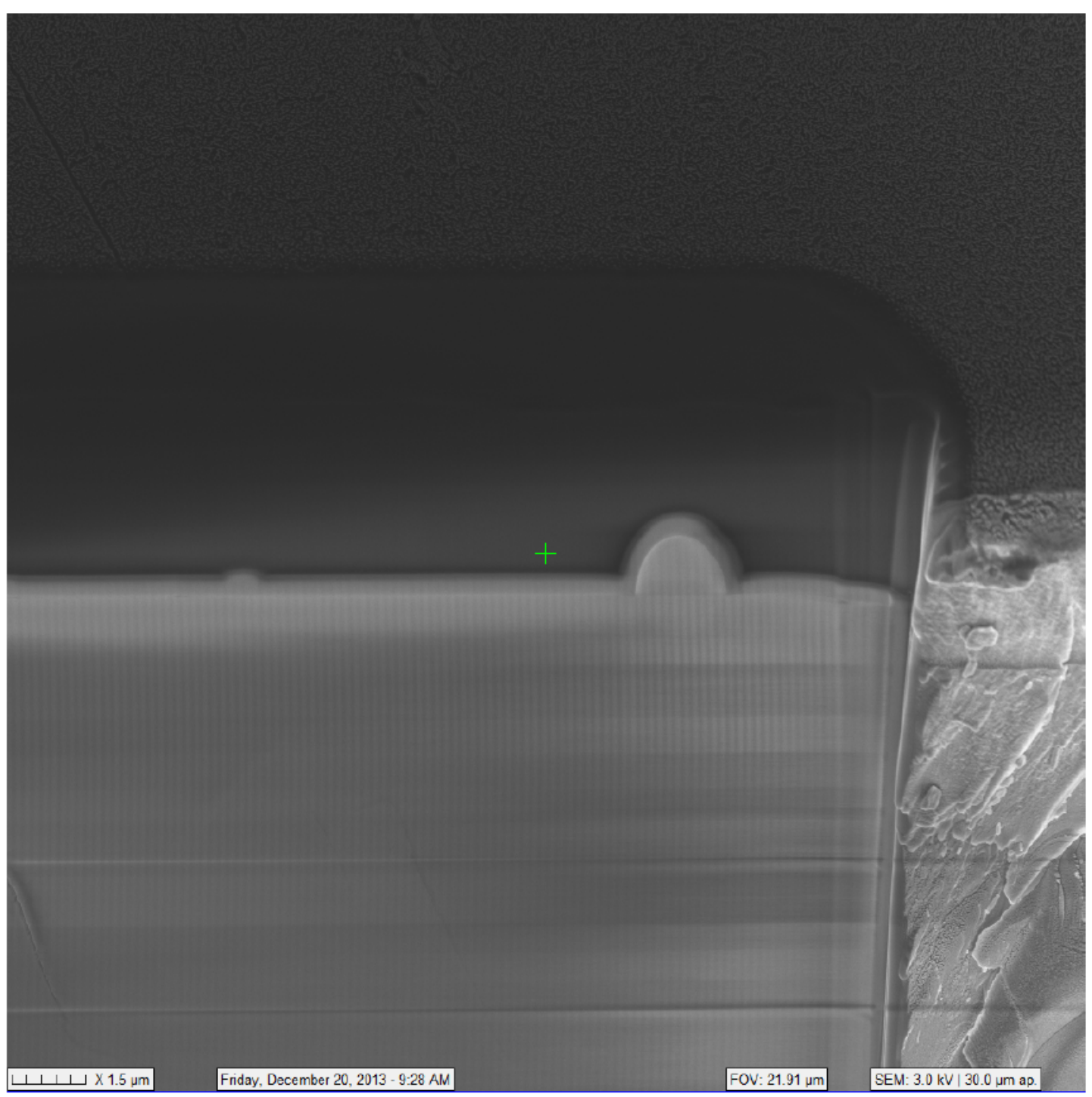

Figure 6.15: Fibics SEM image of "chicken pox" bubbles. Die were cleaved using a Galium beam to allow visual inspection of die using a "clean cut".

\subsection{Comparison to Published Results}

The comparison paper [1] implemented an 8-bit charge sharing SAR ADC using 28 unit capacitors. The charge sharing SAR ADC presented in this work used 15 unit capacitors.

A comparison of the performance metrics for the $\mathrm{SAR}$ ADC presented in $[1]$ and in this work are presented in Table 6.1. [1]'s INL and DNL are metrics from simulation. [1]'s ENOB, signal to noise and distortion ratio SNDR, and SFDR are measured values.

[1]'s ENOB, SNDR, and SFDR results from simulation are not available. Therefore, a direct comparison of these parameters for this work to [1]'s isn't possible. However, [1] 


\begin{tabular}{|l|l|l|}
\hline Metric $\left(\mathrm{TT},+27^{\circ} \mathrm{C}\right)$ & {$[1]$} & This Work \\
\hline ENOB & $4.5 \mathrm{bits}$ & $7.8 \mathrm{bits}$ \\
\hline INL & $2 \mathrm{LSB}$ & $0.3069 \mathrm{LSB}$ \\
\hline DNL & $1 \mathrm{LSB}$ & $0.3627 \mathrm{LSB}$ \\
\hline SNDR & $25 \mathrm{~dB}$ & $49.9 \mathrm{~dB}$ \\
\hline SFDR & $42 \mathrm{~dB}$ & $60.4 \mathrm{~dB}$ \\
\hline
\end{tabular}

Table 6.1: Performance comparison between [1] and this work.

did provide $\mathrm{DNL}$ and $\mathrm{INL}$ from simulation so a comparison with these metrics can be done. [1]'s design has DNL and INL values that are both greater than 1/2 LSB. Therefore, [1]'s design has missing codes. The design in this work has DNL and INL values less than $1 / 2$ LSB. The reason for the poor performance of [1]'s manufactured design is most likely due to the fact that the capacitors in the capacitor array were not laid out using common centroid layout with dummy capacitors. Therefore, each capacitor in [1]'s capacitor array will not have the same capacitance and the dynamic performance parameters DNL and INL are poor. This work used proper layout techniques (common centroid and dummy capacitors) to achieve its performance so a comparison of layout area between [1] and this work a good metric.

The improved DNL, INL, and number of capacitors used to implement the capacitor array in this work show that this work has significant improvement over [1]. 


\section{Chapter 7}

\section{Conclusion}

\subsection{Contribution to research}

Telemonitoring systems that measure signals from patients in every day situations allow physicians in locations remote from their patients to accurately diagnose their patient's health issues. The ability for remote diagnosis means that increasingly scarce medical resources can be used more cost effectively. The key to an accurate diagnosis are telemonitoring systems that don't impede a patent's mobility, are physically small, and have a long battery life.

Test modalities such as EEG, ECG, EMG, and ENG measure frequencies that are less than 1kHz. Therefore, low resolution SAR ADC [1] which are known for their high energy efficiency [3] are a good match for telemonitoring systems used by these test modalities.

The charge sharing $\mathrm{SAR} \mathrm{ADC}$ is composed of a $\mathrm{S} / \mathrm{H}$, comparator, capacitor array, and SAR (implemented using digital logic). The low frequencies measured using this charge sharing SAR ADC require a process technology that has low leakage. 130nm process technologies are known to have much better leakage performance than smaller geometries such as $90 \mathrm{~nm}, 65 \mathrm{~nm}, 45 \mathrm{~nm}$, etc. Larger process geometries such as $180 \mathrm{~nm}$ and $350 \mathrm{~nm}$, which have slightly better leakage characteristics than $130 \mathrm{~nm}$, are available from CMC, But, their fabrication schedule didn't line up with the requirements of this 
thesis.

The standard cell library used to implement the SAR logic was developed specifically for this thesis. The standard cell library includes 12-track tall cells such as an asynchronously reset flip-flop, an asynchronously set flip-flop, a two input NAND gate, a two input NOR gate, an inverter, a clock tree buffer, and a filler cell. CMC does offer an ARM standard cell library but this library does not include GDSII views, making simulation with standard simulators such as Spectre not possible. Full chip simulations with the SAR (using cells from the custom standard cell library) connected to the $\mathrm{S} / \mathrm{H}$, comparator, and capacitor array were carried out using Spectre. This library was also used with standard PNR tools to synthesize Verilog RTL, place cells, route nets, and route power nets. This fully placed and routed digital design was then directly imported into the Cadence analog tool environment.

This custom standard cell library, along with its views for standard PNR tools, is available to DOE students for future projects.

A rising edge clocked, comparator based on the StrongARM architecture with calibration was also specifically developed for this thesis. The performance of this comparator was validated over 400 simulations. Where the simulations were generated using IBM CMRF8SF Monte Carlo models to provide verification over process and mismatch.

The $\mathrm{S} / \mathrm{H}$ developed for this thesis allowed for the use of a smaller hold capacitor because the $\mathrm{S} / \mathrm{H}$ architecture took advantage of the "Miller Effect". Smaller hold capacitor means less die area and less leakage power. This $\mathrm{S} / \mathrm{H}$ also used an amplifier designed to operate in the subthreshold region to further reduce power dissipation. The simulated performance of this $\mathrm{S} / \mathrm{H}$ was $\mathrm{SNR}=80.1 \mathrm{~dB}, \mathrm{SINAD}=77.5 \mathrm{~dB}, \mathrm{ENOB}=12.6 \mathrm{bits}$, $\mathrm{THD}=-80.9 \mathrm{~dB}$, and $\mathrm{SFDR}=84.7 \mathrm{~dB}$ at TT process and $27^{\circ} \mathrm{C}$.

The entire charge sharing $[\mathrm{SAR} \mathrm{ADC}$ s performance from simulation was $\underline{\mathrm{SNR}}=49.9$ $\mathrm{dB}, \mathrm{SINAD}=48.7 \mathrm{~dB}, \mathrm{ENOB}=7.8 \mathrm{bits}, \mathrm{THD}=-60.3 \mathrm{~dB}$, and $\mathrm{SFDR}=60.3 \mathrm{~dB}$ at TT process and $+27^{\circ} \mathrm{C}$. The worst case $\mathrm{DNL}$ was $0.36 \mathrm{LSB}$ and the worst case INL was 0.31 LSB.

The measurement results of the physical circuit implementation in this thesis do not 
match the predicted simulation results. The measured results have been consistent in packaged and unpackaged die and across multiple testing environments.

It was noticed that there are manufacturing irregularities known as "chicken pox" visible on the die. It is possible that there are further manufacturing defects that aren't clearly visible.

The IBM CMRF8SF library used to design this work does model leakage. This was proven in Section 5.6. Therefore, the differences between the simulated and the measured results are not leakage related. That is of course, assuming that some other manufacturing defect is causing more leakage than is modelled by the library.

However, the improved DNL, INL, and reduced number of capacitors required to implement the capacitor array in this work vs. [1]'s shows that even in simulation, this work has improved upon the literature.

\subsection{Future work}

The charge sharing SAR ADC uses a digital algorithm to control a capacitor array to convert an analog input signal into a digital equivalent. Increasing the number of bits of this charge sharing SAR ADC could possibly be done by only increasing the number of standard cells used by the SAR digital logic. The SAR logic standard cell utilization in the current implementation is only about $25 \%$. Therefore, the number of standard cells can easily be doubled or even tripled before growing the standard cell placement area. The factor driving an increase in the area required to place the SAR logic standard cells is the routing (as long as the area required by the standard cells is less than $100 \%$ utilization). It is commonly found that designs becomes unroutable using standard place and route routers available from EDA vendors such as Cadence and Synopsys as placement utilization increases beyond $75 \%$.

Migrating this design to a $65 \mathrm{~nm}$ process will reduce the area required to implement the SAR s standard cell logic. However, as previously mentioned, the SAR standard cell logic utilization is very low so an area reduction of this circuit will not decrease the size of 
the die. The real driving factor will be the area required to implement capacitors. This will depend on the specific $65 \mathrm{~nm}$ process used.

It is well known that $65 \mathrm{~nm}$ designs suffer from much larger leakage currents than $130 \mathrm{~nm}$ designs. Therefore, migrating to $65 \mathrm{~nm}$ will result in higher power dissipation if this design is directly ported from $130 \mathrm{~nm}$ to $65 \mathrm{~nm}$. 
Appendix A

Detailed Binary Weighted SAR

ADC Conversion Example 


\section{A.1 Step 1: Sampling Mode}

Figure A.1 shows the binary weighted SAR ADC in "sampling mode". This is the configuration where the ADC samples the analog input signal. As Figure A.1 shows, the top plate of the capacitors in the array are connected to ground. The bottom plate of all capacitors in the array are connected to the input voltage $\left(V_{\text {in }}\right)$. The voltage at node $\mathrm{Vx}$ is therefore set to voltage $V_{\text {in }}$.

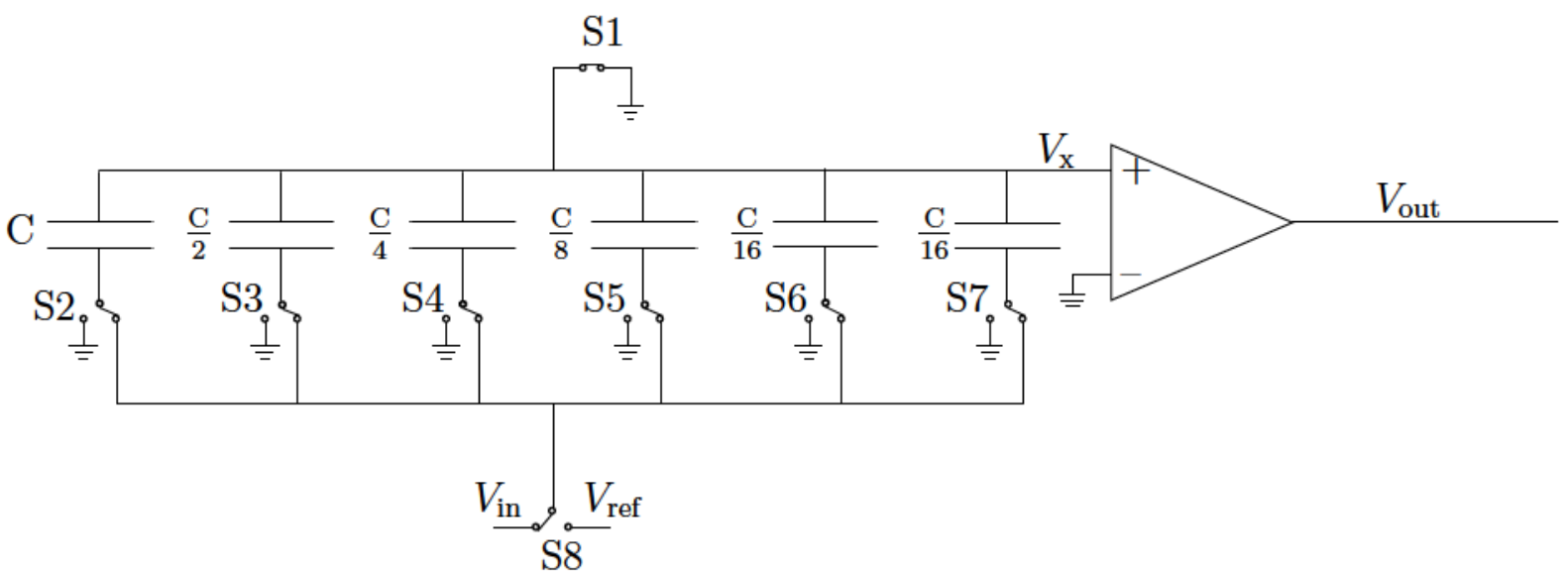

Figure A.1: Binary weighted SAR ADC schematic diagram shown in the signal sampling configuration. Switch S1 connects the top plate of the capacitors in the capacitor array to ground. Switches S2, S3, S4, $\mathrm{S} 5, \mathrm{~S} 6, \mathrm{~S} 7$, and S8 connect the bottom plates of the capacitors in the capacitor array to $V_{\text {in }}$.

\section{A.2 Step 2: Hold Mode}

The bottom plate of all the capacitors in the array are connected to ground and the top plate of all these capacitors are disconnected from ground. This causes the voltage at node $V_{\mathrm{x}}$ to become $-V_{\text {in }}$. The configuration of the binary weighted SAR ADC for this mode is shown in Figure A.2.

\section{A.3 Step 3: Determine binary value of bit 4}

The bottom plate of the capacitor associated with the most significant bit, bit 4 , is connected to Vref. The value of this capacitor is half the total capacitance in the array. The remaining capacitors in the array are connected in parallel and the sum of their capacitance is half that of the total capacitance array. Therefore, a voltage divider is 


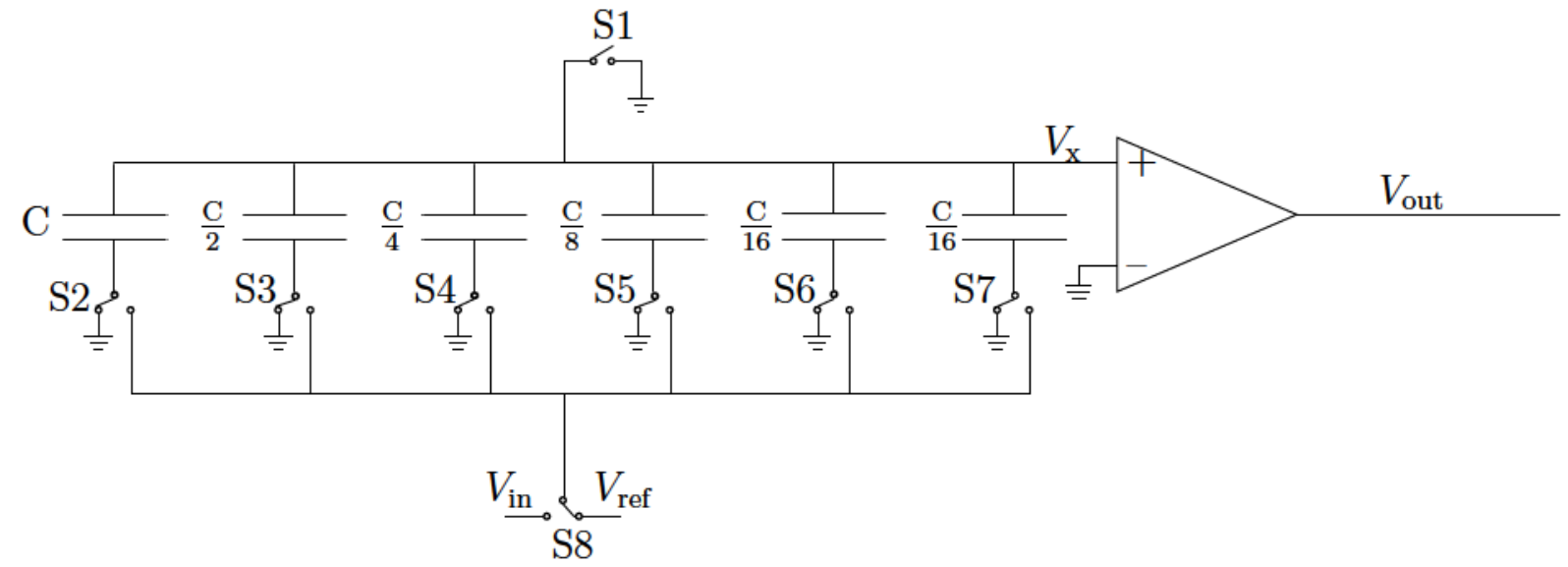

Figure A.2: Binary weighted SAR ADC schematic diagram shown in the "hold" configuration. Switch $\mathrm{S} 1$ is opened so the top plates of the capacitors in the capacitor array are connected to the comparator's non-inverting input and not to ground. Switches S2, S3, S4, S5, S6, and S7 connect the bottom plates of the capacitors in the capacitor array to ground.

created such that the voltage across the capacitor associated with bit 4 is one half of

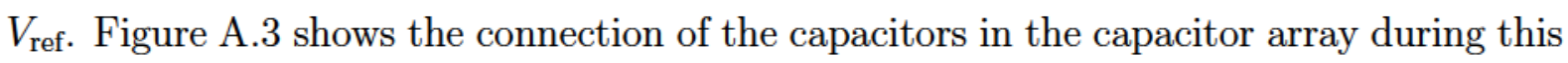
phase.

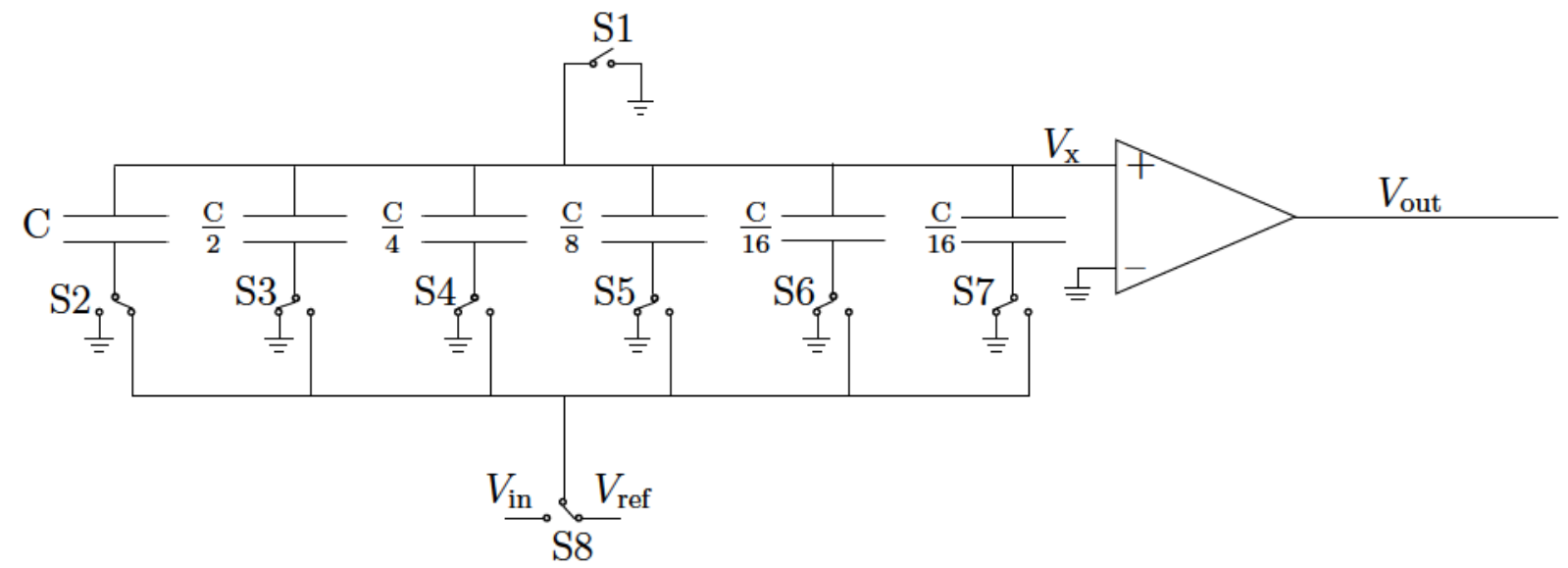

Figure A.3: Binary weighted SAR ADC schematic diagram showing the circuit configuration used to determine the logic value of bit 4 by allowing capacitor "C" to charge. Switch S1 remains opened so the top plates of the capacitors in the capacitor array are connected to the comparator's non-inverting input and not to ground. Switch S2 connects capacitor C's bottom plate to $V_{\text {ref. }}$ Switches S3, S4, S5, S6, and $\mathrm{S} 7$ connect the bottom plates of capacitors $\frac{\mathrm{C}}{2}, \frac{\mathrm{C}}{4}, \frac{\mathrm{C}}{8}$, and $\frac{\mathrm{C}}{16}$ to ground.

As a result of the circuit configuration shown in Figure A.3, the voltage across the capacitor associated with bit 4 is added to the voltage at node $V_{\mathrm{x}}$. Therefore, at this point of the conversion, the voltage at node $V_{\mathrm{x}}$ is equal to $-V_{\text {in }}+\frac{V_{\text {ref }}}{2}$. For the example shown in Figure 2.2, the binary value of bit 4 is logic zero due to the fact that the voltage at node $V_{\mathrm{x}}$ is negative. 


\section{A.4 Step 4: Determine binary value of bit 3}

The bottom plate of the capacitor associated with the most significant bit, bit 4 is connected to ground and the bottom plate of the next most significant bit, bit 3 is connected to $V_{\text {ref }}$. Since the voltage divider created by the capacitance is such that the capacitance of the capacitor associated with bit 3 is one quarter of the total capacitance in the capacitor array, the voltage across the capacitor associated with bit 3 is one quarter of $V_{\text {ref. }}$ This is shown in Figure A.4.

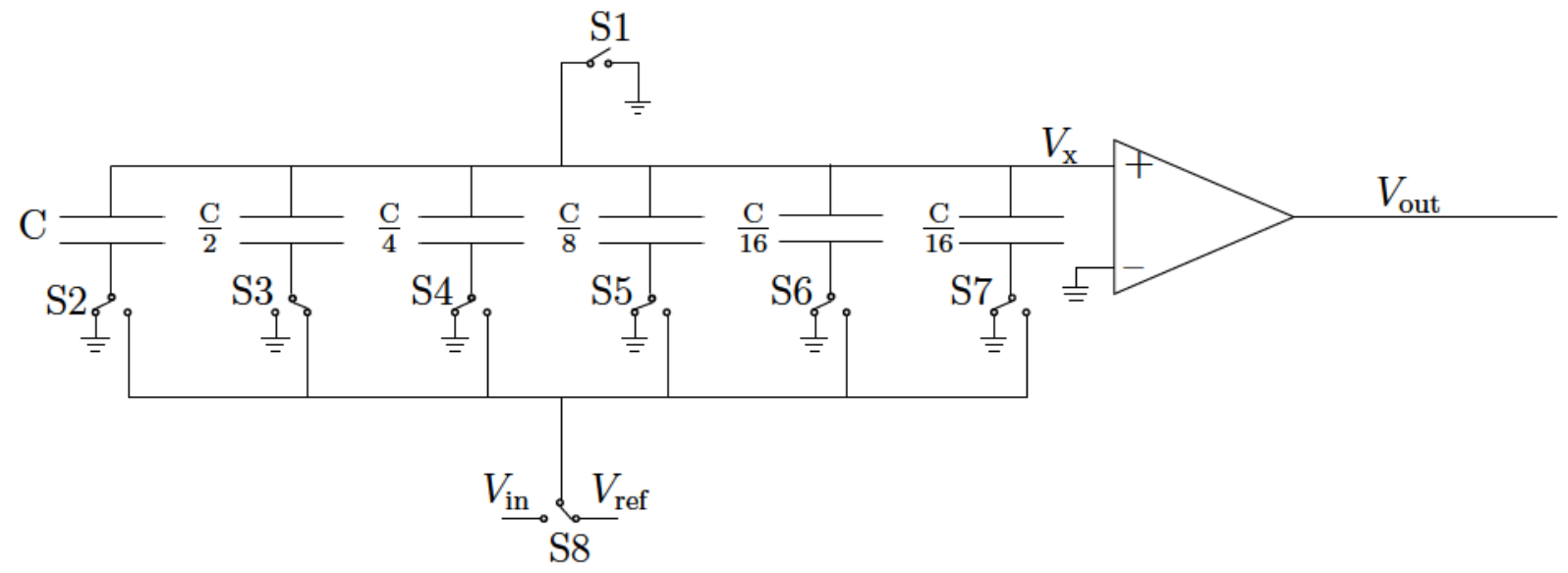

Figure A.4: Binary weighted SAR ADC schematic diagram showing the circuit configuration used to determine the logic value of bit 3 by allowing capacitor $\frac{\mathrm{C}}{2}$ to charge. Switch S1 remains opened so the top plates of the capacitors in the capacitor array are connected to the comparator's non-inverting input and not to ground. Switch S2 connects capacitor C's bottom plate to ground. Switch S3 connects capacitor $\frac{\mathrm{C}}{2}$ 's bottom plate to $V_{\text {ref. }}$. Switches $\mathrm{S} 4, \mathrm{~S} 5, \mathrm{~S} 6$, and $\mathrm{S} 7$ connect the bottom plates of capacitors $\frac{\mathrm{C}}{4}, \frac{\mathrm{C}}{8}$, and $\frac{\mathrm{C}}{16}$ to ground.

The result of the circuit configuration shown in Figure A.4 is that the voltage across the capacitor associated with bit 3 is added to the voltage at node $V_{\mathrm{x}}$. Therefore, the

voltage at node $V_{\mathrm{x}}$ is equal to $-V_{\mathrm{in}}+\frac{V_{\text {ref }}}{2}+\frac{V_{\text {ref }}}{4}$. In the case of the example shown in Figure 2.2, the voltage at node $V_{\mathrm{x}}$ is positive so bit 3 is set to binary 1 .

\section{A.5 Step 5: Determine binary value of bit 2}

The bottom plate of the capacitor associated with bit 3 is connected to ground and the bottom plate of the capacitor associated with bit 2 is connected to $V_{\text {ref. }}$ This time, the voltage divider is such that the capacitor associated with bit 2 is one eighth of Vref. The circuit configuration shown in Figure A.5 shows the connections for this configuration. 
For the example shown in Figure A.5, the voltage at node $V_{\mathrm{x}}$ is equal to $-V_{\text {in }}+\frac{V_{\text {ref }}}{2}+\frac{V_{\text {ref }}}{4}$ $+\frac{V_{\text {ref }}}{8}$, which is positive, so bit 2 is set to binary 1 .

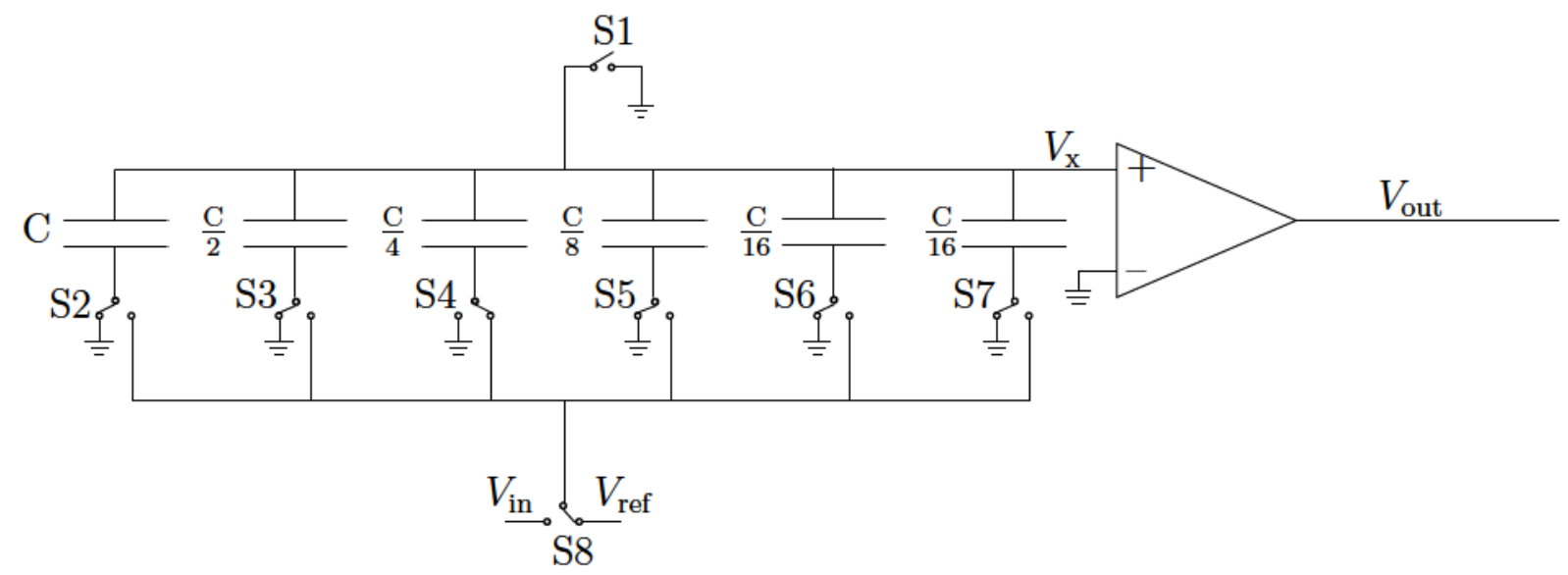

Figure A.5: Binary weighted SAR ADC schematic diagram showing the circuit configuration used to determine the logic value of bit 2 by allowing capacitor $\frac{\mathrm{C}}{4}$ to charge. Switch S1 remains opened so the top plates of the capacitors in the capacitor array are connected to the comparator's non-inverting input and not to ground. Switch S3 connects capacitor $\frac{\mathrm{C}}{2}$ 's bottom plate to ground. Switch S4 connects capacitor $\frac{\mathrm{C}}{4}$ 's bottom plate to $V_{\text {ref. }}$ Switches S2, S5, S6, and S7 connect the bottom plates of capacitors C, $\frac{\mathrm{C}}{8}$, and $\frac{\mathrm{C}}{16}$ to ground.

\section{A.6 Step 6: Determine value of bit 1}

At this step of the conversion, the bottom plate of the capacitor associated with bit 2 is connected to ground and the bottom plate of the capacitor associated with bit 1 is connected to $V_{\text {ref. }}$ In this case, the voltage divider causes the voltage across the capacitor associated with bit 1 to be one sixteenth of $V_{\text {ref. }}$ This is shown in Figure A.6. For the example shown in Figure 2.2 , the voltage at node $V_{\mathrm{x}}$ is equal to $-V_{\text {in }}+\frac{V_{\text {ref }}}{4}+\frac{V_{\text {ref }}}{8}+\frac{V_{\text {ref }}}{16}$, which, since node $V_{\mathrm{x}}$ is positive, causes bit 1 to be a binary 1 . 


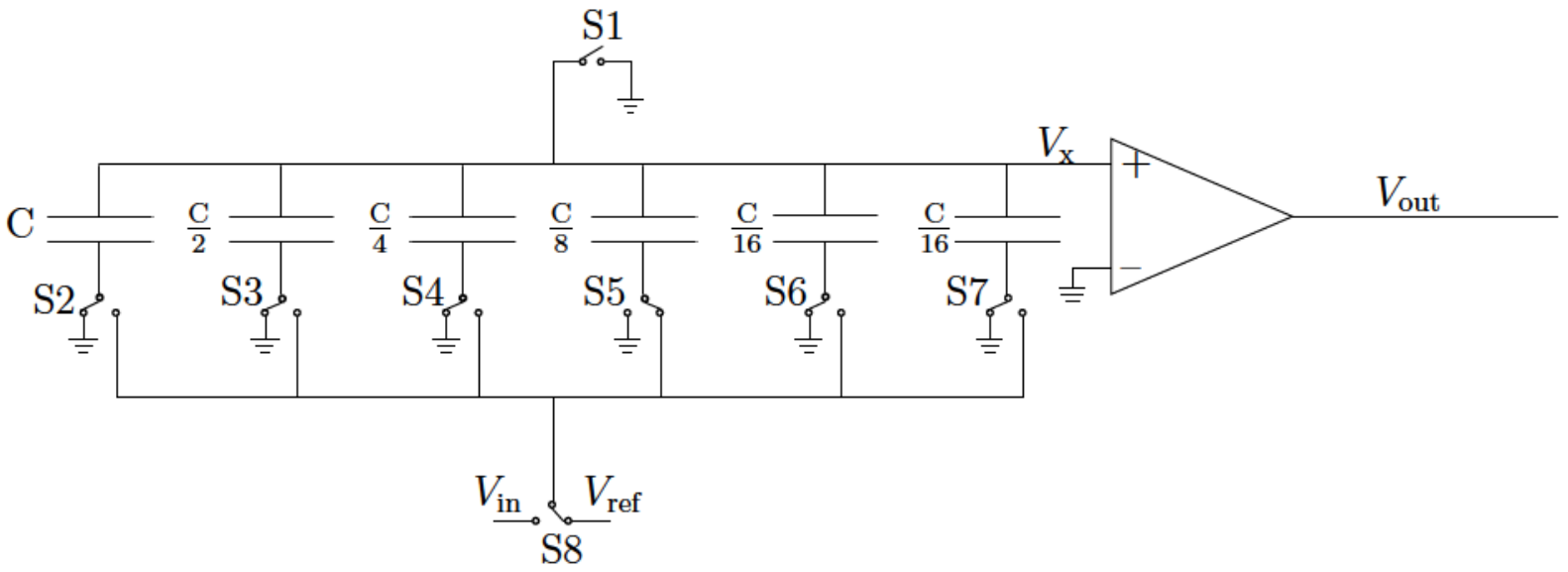

Figure A.6: Binary weighted SAR ADC schematic diagram showing the circuit configuration used to determine the logic value of bit 1 by allowing capacitor $\frac{C}{8}$ to charge. Switch S1 remains opened so the top plates of the capacitors in the capacitor array are connected to the comparator's non-inverting input and not to ground. Switch S4 connects capacitor $\frac{\mathrm{C}}{4}$ 's bottom plate to ground. Switch S5 connects capacitor $\frac{\mathrm{C}}{8}$ 's bottom plate to $V_{\text {ref. }}$. Switches $\mathrm{S} 2, \mathrm{~S} 3, \mathrm{~S} 6$, and $\mathrm{S} 7$ connect the bottom plates of capacitors $\mathrm{C}, \frac{\mathrm{C}}{2}$, and $\frac{\mathrm{C}}{16}$ to ground.

\section{A.7 Step 7: Determine value of bit 0}

Here, the bottom plate of the capacitor associated with bit 1 is connected to ground and the bottom plate of the capacitor associated with bit 0 connected to $V_{\text {ref. }}$ The circuit configuration is shown in Figure A.7. For the example shown in Figure 2.2, the voltage at node $V_{\mathrm{x}}$ is equal to $-V_{\text {in }}+\frac{V_{\text {ref }}}{4}+\frac{V_{\text {ref }}}{8}+\frac{V_{\text {ref }}}{16}+\frac{V_{\text {ref }}}{32}$, which, since the voltage at node $V_{\mathrm{x}}$ is positive - causes bit 0 to be set to binary 1 .

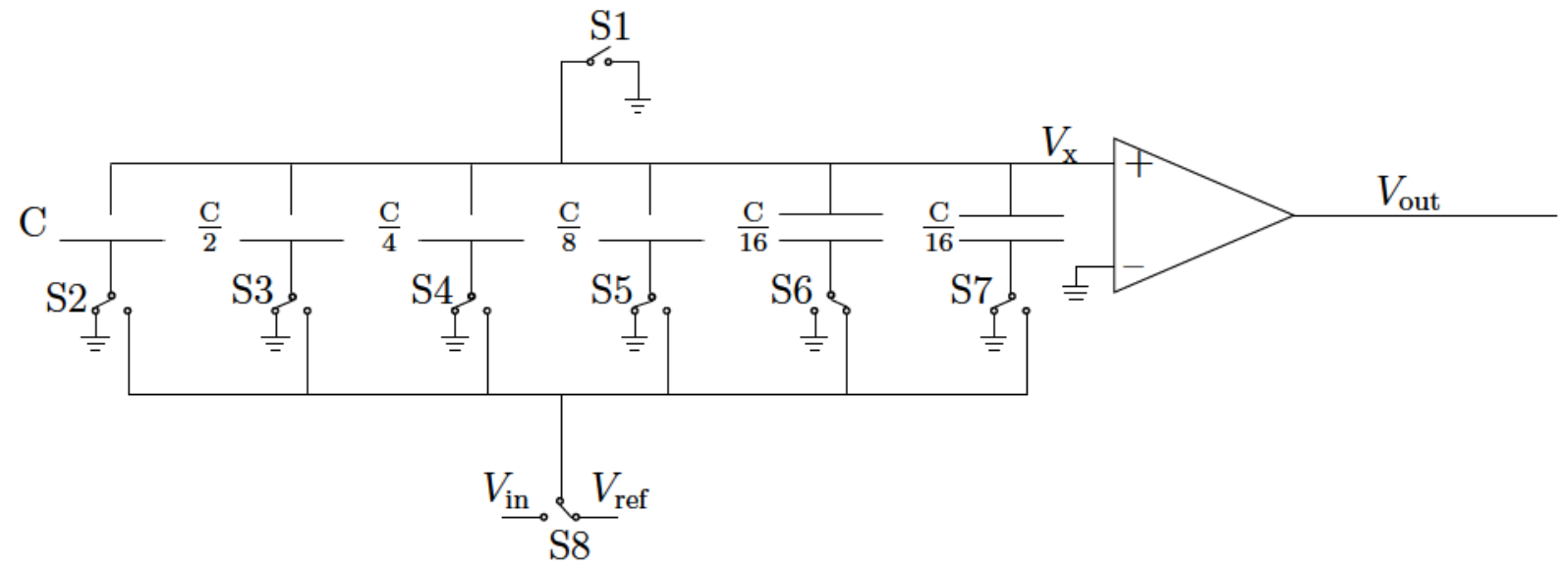

Figure A.7: Binary weighted SAR ADC schematic diagram showing the circuit configuration used to determine the logic value of bit 0 by allowing capacitor $\frac{\mathrm{C}}{16}$ to charge. Switch S1 remains opened so the top plates of the capacitors in the capacitor array are connected to the comparator's non-inverting input and not to ground. Switch S5 connects capacitor $\frac{\mathrm{C}}{8}$ 's bottom plate to ground. Switch S6 connects capacitor $\frac{\mathrm{C}}{16}$ 's bottom plate to $V_{\text {ref. }}$. Switches S2, S3, S4, and S7 connect the bottom plates of capacitors $\mathrm{C}, \frac{\mathrm{C}}{2}, \frac{\mathrm{C}}{4}$, and $\frac{\mathrm{C}}{16}$ to ground.

The digital equivalent of the sampled analog waveform is 01111. 
136APPENDIX A. DETAILED BINARY WEIGHTED SAR ADC CONVERSION EXAMPLE 


\section{Appendix B}

\section{Detailed Serial SAR ADC \\ Conversion Example}




\section{B.1 Step 1: Determine MSB of ADC's Digital Out- put}

The first half of the determination of the $\mathrm{ADC}$ s most significant bit (MSB) is shown in Figure B.1. As this figure shows, switches $S_{2}$ and $S_{4}$ are closed, charging $C_{1}$ to $V_{\text {ref }}$ and discharging $\mathrm{C}_{2}$.

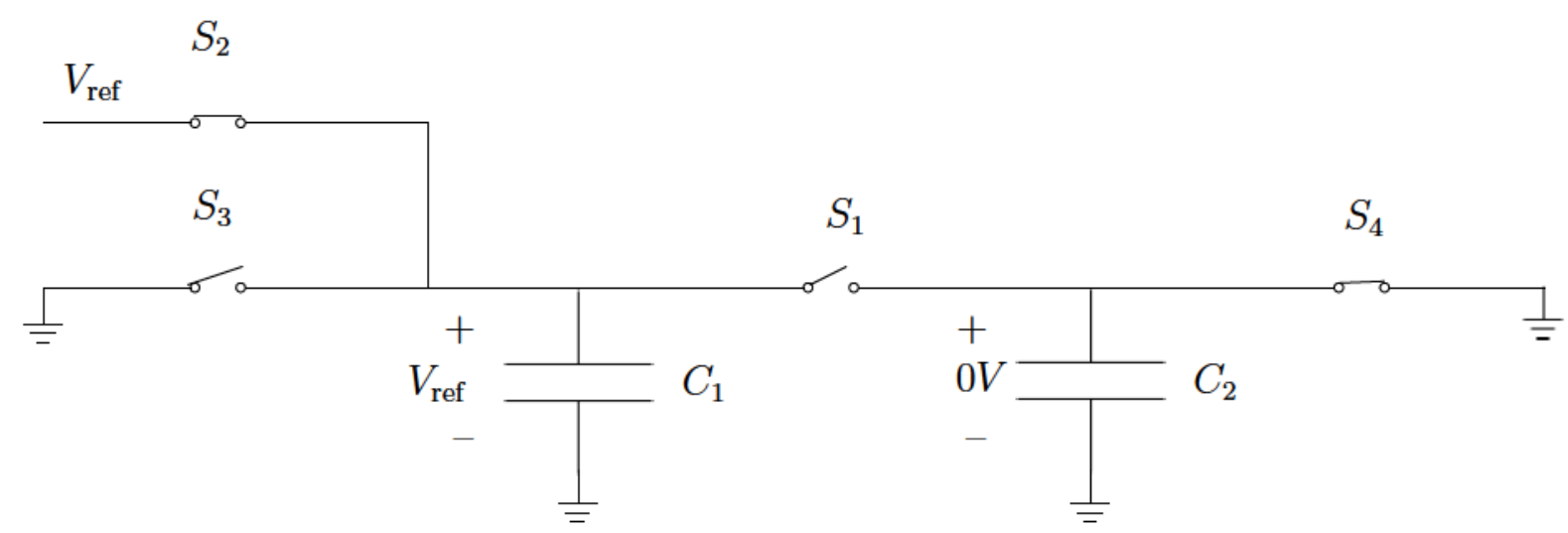

Figure B.1: Serial DAC schematic diagram showing the circuit configuration when capacitor $C_{1}$ is charged to $V_{\text {ref }}$ and $C_{2}$ is discharged to $0 \mathrm{~V}$. This configuration is the first part of the determination of the MSB when the amplitude of the sampled analog signal is slightly greater than $\frac{13}{16} V_{\text {ref }}$.

The second half of the determination of the $\mathrm{ADC}$ s $\mathrm{MSB}$ is shown in Figure B.2. Capacitor $C_{1}$ is connected in parallel with capacitor $C_{2}$, generating a voltage across each capacitor equal to $\frac{V_{\text {ref }}}{2}$. The full serial ADC compares the output of the serial DAC, $\frac{V_{\text {ref }}}{2}$, with the sampled analog signal. The $\mathrm{MSB}$ of the serial $\mathrm{ADC}$ is set to a logic one because $\frac{V_{\text {ref }}}{2}$ is less than the amplitude of the sampled analog signal (the amplitude of the sampled analog signal is slightly greater than $\frac{13}{16} V_{\text {ref }}$ ).

\section{B.2 Step 2: Determine the Second MSB of ADC's Digital Output}

The first part of the second step in the digital to analog conversion of the analog signal with amplitude slightly greater than $\frac{13}{16} V_{\text {ref }}$ is to determine the digital value of the second MSB by first generating $\frac{V_{\text {ref }}}{2}$. The circuit configuration required to generate this voltage is shown in Figure B.1 and Figure B.2. 


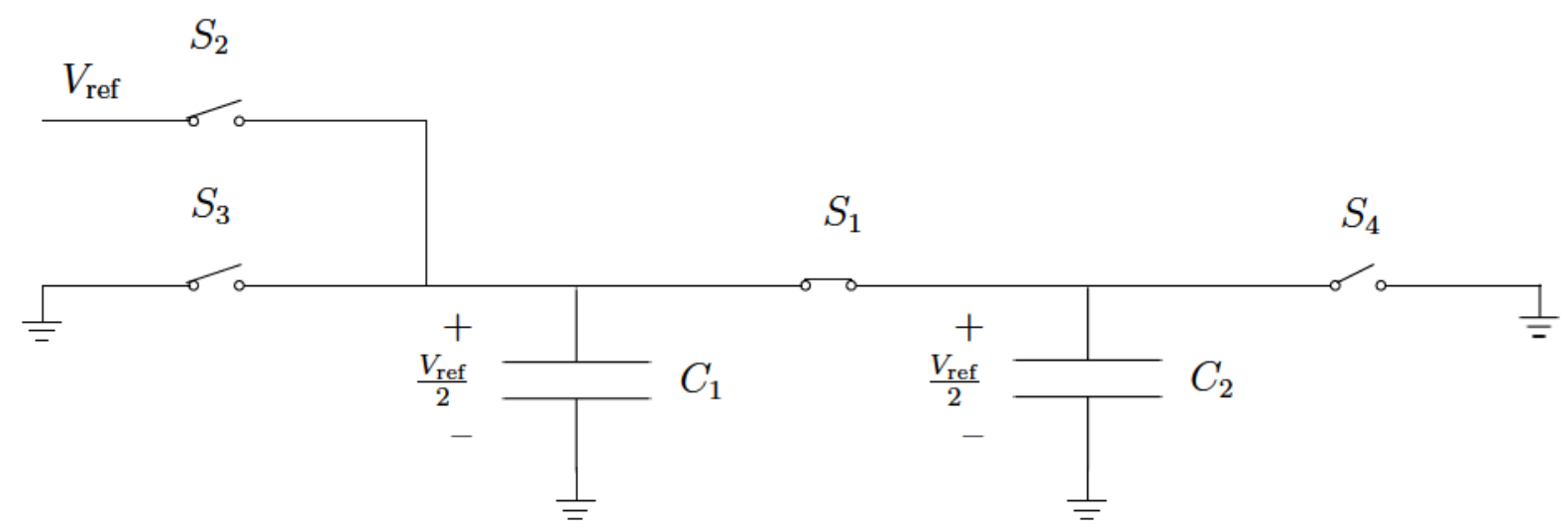

Figure B.2: Serial DAC schematic diagram showing the circuit configuration when capacitor $C_{1}$ is connected in parallel with capacitor $C_{2}$, charging each capacitor to $\frac{V_{\text {ref }}}{2}$. This configuration is the second part of the determination of the MSB when the amplitude of the sampled analog signal is slightly greater than $\frac{13}{16} V_{\text {ref. }}$.

The next step is to generate $\frac{3}{4} V_{\text {ref. }}$ The first part of generating $\frac{3}{4} V_{\text {ref }}$ is shown in Figure B.3. The figure shows switch $S_{2}$ closed, charging $C_{1}$ to $V_{\text {ref. }}$

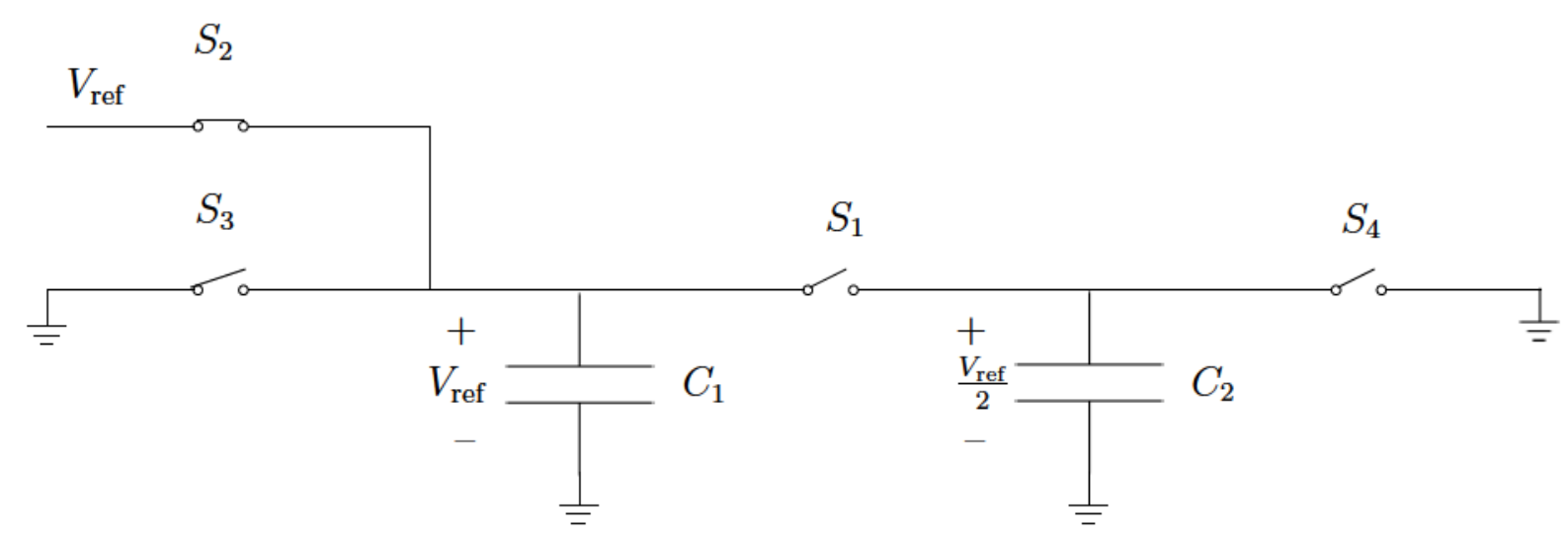

Figure B.3: Serial DAC schematic diagram showing the circuit configuration when capacitor $C_{1}$ is charged to $V_{\text {ref. }}$ This configuration is the first part of the determination of the second MSB when the amplitude of the sampled analog signal is slightly greater than $\frac{13}{16} V_{\text {ref }}$.

The second part of generating $\frac{3}{4} V_{\text {ref }}$ is shown in Figure B.4. Since the voltage across $C_{1}$ is $V_{\text {ref }}$ and capacitor $C_{2}$ is $\frac{V_{\text {ref }}}{2}$, when switch $S_{1}$ is closed, the total voltage across each capacitor becomes $\frac{3}{4} V_{\text {ref. }}$

The second MSB is set to a logic one since $\frac{3}{4} V_{\text {ref }}$ is less than the amplitude of the sampled analog signal. 


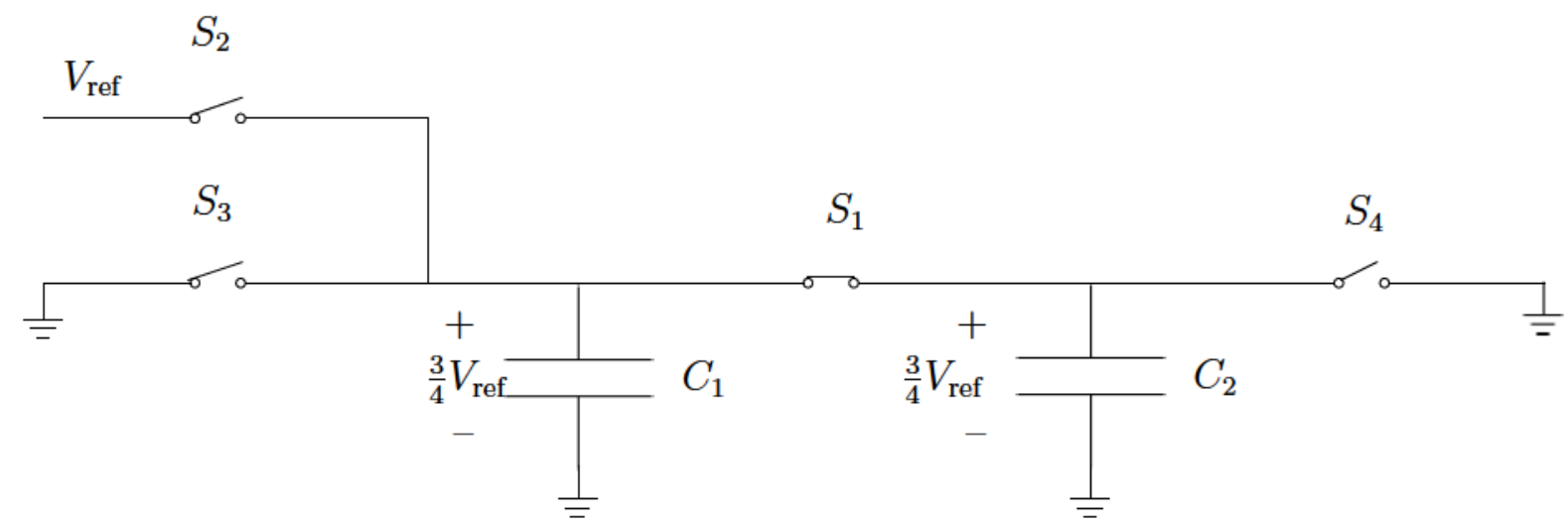

Figure B.4: Serial DAC schematic diagram showing the circuit configuration when capacitor $C_{1}$ is connected in parallel with capacitor $C_{2}$ to create a voltage across each capacitor of $\frac{3}{4} V_{\text {ref. }}$ This configuration is the second part of the determination of the second $\mathrm{MSB}$ when the amplitude of the sampled analog signal is slightly greater than $\frac{13}{16} V_{\text {ref }}$.

\section{B.3 Step 3: Determine the Third MSB of ADC's}

\section{Digital Output}

This step generates $\frac{7}{8} V_{\text {ref. }}$ This is accomplished by generating $\frac{V_{\text {ref }}}{2}, \frac{3}{4} V_{\text {ref }}$, and then generating $\frac{7}{8} V_{\text {ref }}$.

Figure B.1 and Figure B.2 show the circuit configurations required to generate $\frac{V_{\text {ref }}}{2}$. Figure B.3 and Figure B.4 show the circuit configurations required to generate $\frac{3}{4} V_{\text {ref. }}$.

Figure B.5 shows the first circuit configuration of the Serial DAC required to generate $\frac{7}{8} V_{\text {ref. }}$ Switch $S_{2}$ is closed, connecting capacitor $C_{1}$ to $V_{\text {ref. }}$

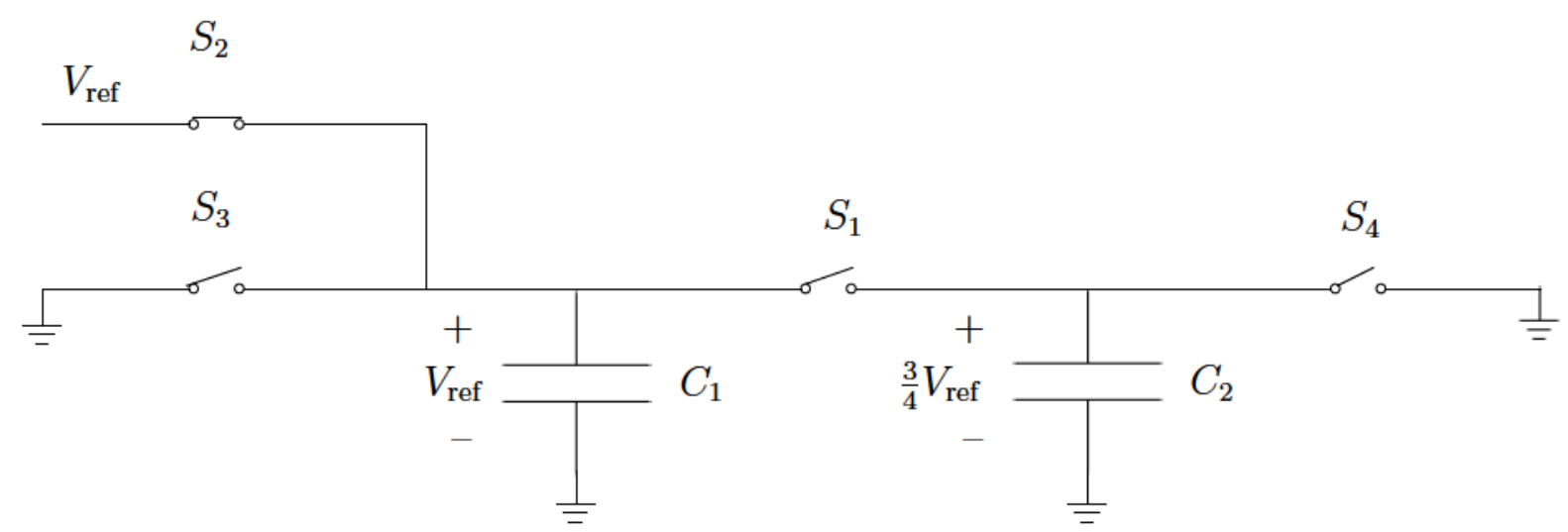

Figure B.5: Serial DAC schematic diagram showing the circuit configuration when capacitor $C_{1}$ is connected to $V_{\text {ref. }}$. This configuration is the first part of the determination of the third MSB when the amplitude of the sampled analog signal is slightly greater than $\frac{13}{16} V_{\text {ref }}$.

Figure B.6 shows the second circuit configuration required to generate $\frac{7}{8} V_{\text {ref. }}$ As this figure shows, when capacitors $C_{1}$ and $C_{2}$ are connected in parallel, the voltage across 
each capacitor becomes $\frac{7}{8} V_{\text {ref. }}$

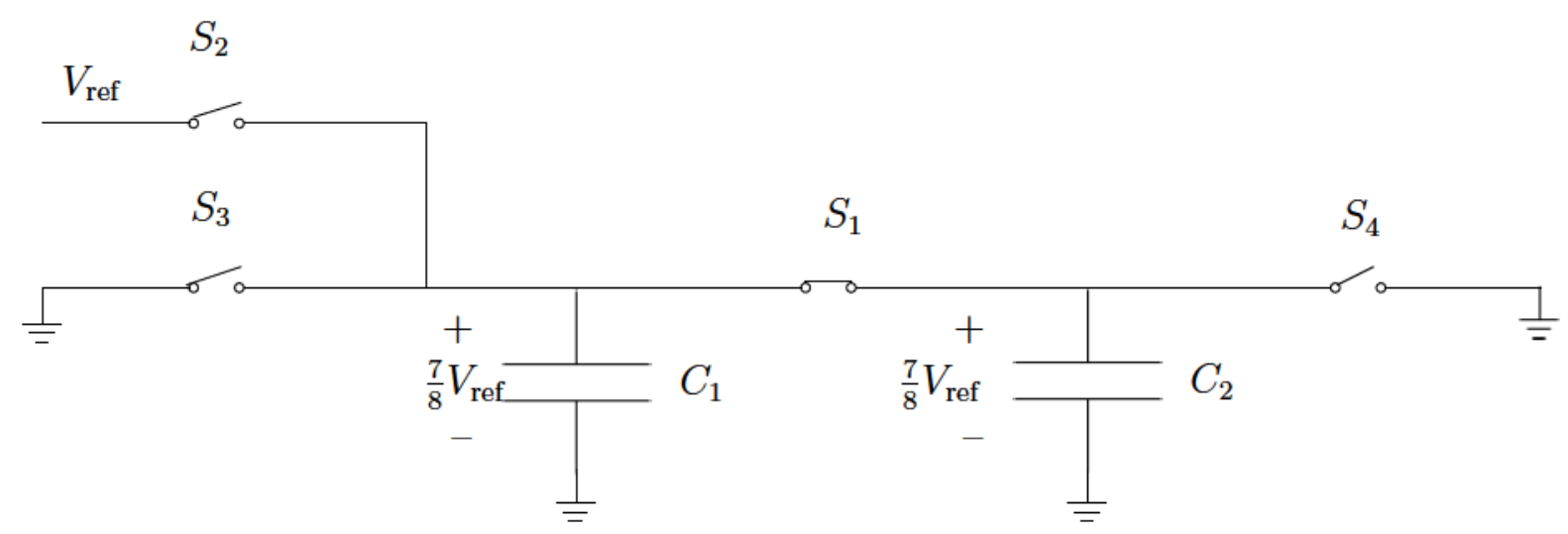

Figure B.6: Serial DAC schematic diagram showing the circuit configuration when capacitors $C_{1}$ and $C_{2}$ are connected in parallel. The voltage across each capacitor will be $\frac{7}{8} V_{\text {ref }}$. This configuration is the second part of the determination of the third MSB when the amplitude of the sampled analog signal is slightly greater than $\frac{13}{16} V_{\text {ref. }}$.

The third MSB is set to a logic zero because $\frac{7}{8} V_{\text {ref }}$ is greater than the sampled analog signal with amplitude slightly greater than $\frac{13}{16} V_{\text {ref. }}$

\section{B.4 Step 4: Determine the Fourth MSB of ADC's Digital Output}

This step is to generate $\frac{13}{16} V_{\text {ref. }}$ This step of the algorithm is different than the previous three steps because the generated voltage $\frac{7}{8} V_{\text {ref }}$ was greater than the amplitude of the sampled signal, which is slightly greater than $\frac{13}{16} V_{\text {ref. }}$ The result was that the third most significant bit was set to logic zero. The voltage compared to the sampled analog signal for this stage of the conversion is generated as described in the following text in this section.

First, generate $\frac{V_{\text {ref }}}{2}$ as is shown in Figure B.1 and Figure B.2. Next, generate $\frac{V_{\text {ref }}}{4}$ as shown in Figure B.7 and Figure B.8.

Next, generate $\frac{5}{8} V_{\text {ref. }}$ This voltage is generated as shown in Figure B.9 and Figure B.10.

The last step is to generate $\frac{13}{16} V_{\text {ref. }}$ This is shown in Figure B.11 and Figure B.12.

The Fourth $\mathrm{MSB}$ is set to a logic one because the sampled analog voltage is, in reality, slightly greater than $\frac{13}{16} V_{\text {ref. }}$ Therefore, the complete 4-bit digital value is 1101 . This algorithm required 20 steps to generate the digital value of these four bits. 


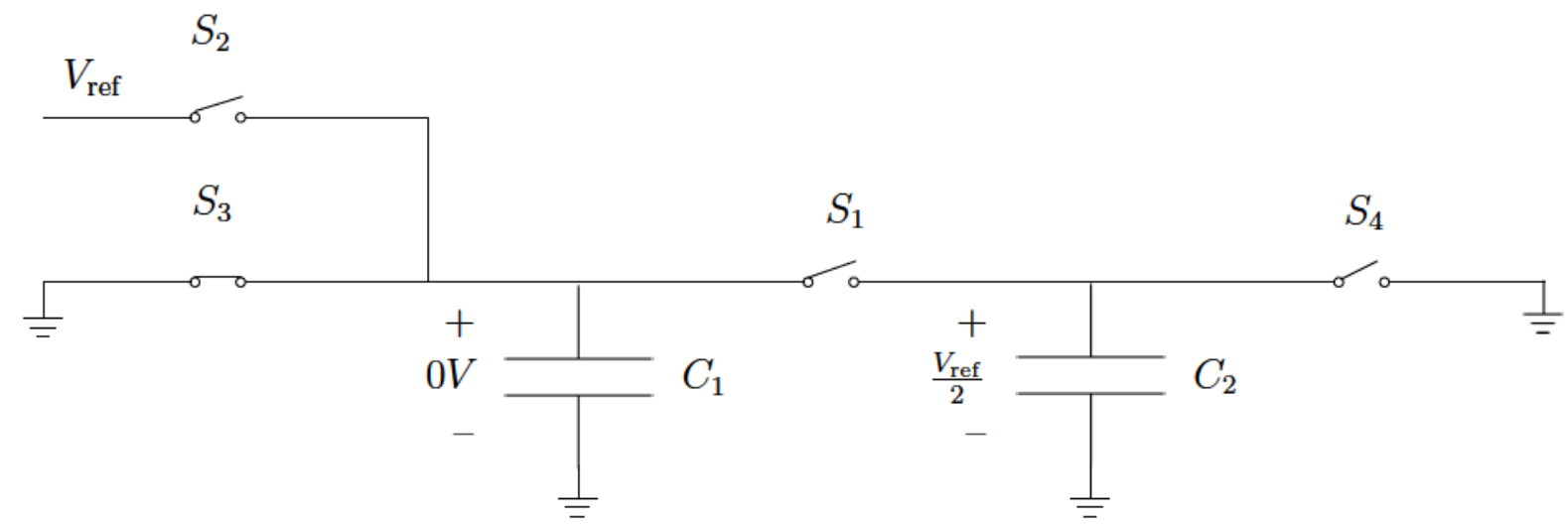

Figure B.7: Serial DAC schematic diagram showing the circuit configuration when capacitor $C_{1}$ is connected to ground and $C_{1}$ discharges to $0 \mathrm{~V}$. The voltage across capacitor $C_{2}$ is still $\frac{V_{\text {ref }}}{2}$ from the previous step. This configuration is the first part of the determination of the fourth $\mathrm{MSB}$ when the amplitude of the sampled analog signal is slightly greater than $\frac{13}{16} V_{\text {ref }}$.

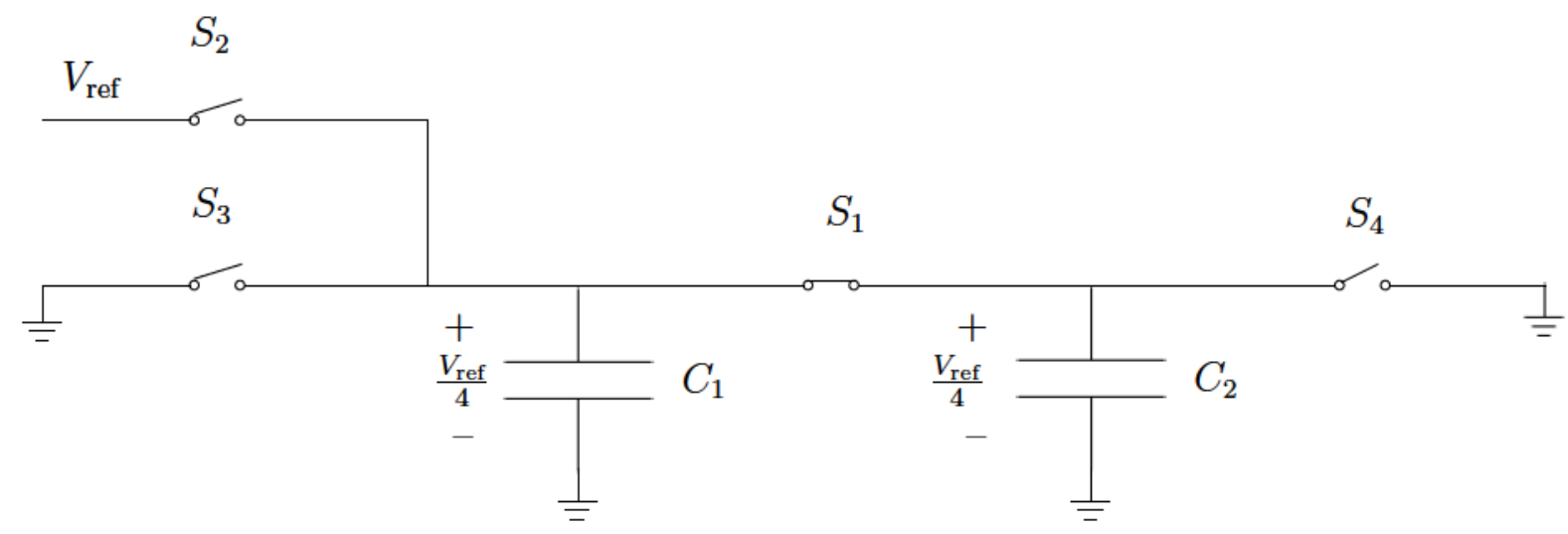

Figure B.8: Serial DAC schematic diagram showing the circuit configuration when capacitors $C_{1}$ and $C_{2}$ are connected in parallel. The voltage across each capacitor is $\frac{V_{\text {ref }}}{4}$. This configuration is the second part of the determination of the fourth MSB when the amplitude of the sampled analog signal is slightly greater than $\frac{13}{16} V_{\text {ref. }}$.

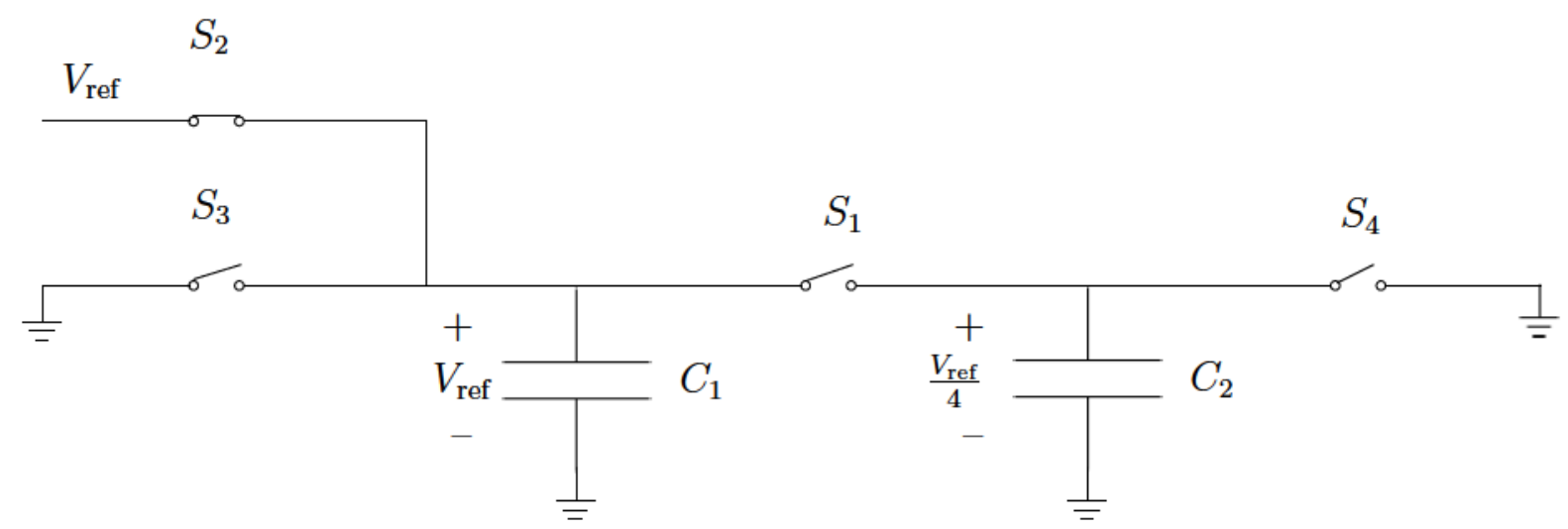

Figure B.9: Serial DAC schematic diagram showing the circuit configuration when capacitor $C_{1}$ is connected to $V_{\text {ref. }}$. The voltage across capacitor $C_{2}$ is still $\frac{V_{\text {ref }}}{4}$ as a result of the previous step. This configuration is the third part of the determination of the fourth MSB when the amplitude of the sampled analog signal is slightly greater than $\frac{13}{16} V_{\text {ref. }}$. 


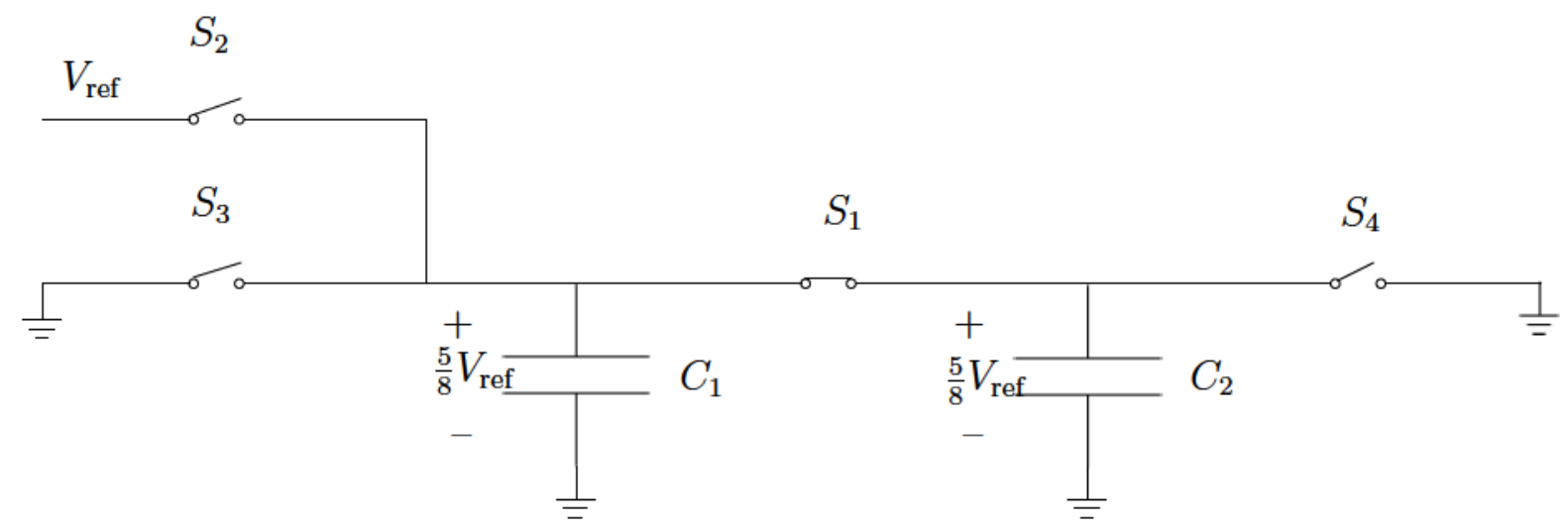

Figure B.10: Serial DAC schematic diagram showing the circuit configuration when capacitors $C_{1}$ and $C_{2}$ are connected in parallel. The voltage across each capacitor becomes $\frac{5}{8} V_{\text {ref. }}$ This configuration is the fourth part of the determination of the fourth MSB when the amplitude of the sampled analog signal is slightly greater than $\frac{13}{16} V_{\text {ref. }}$.

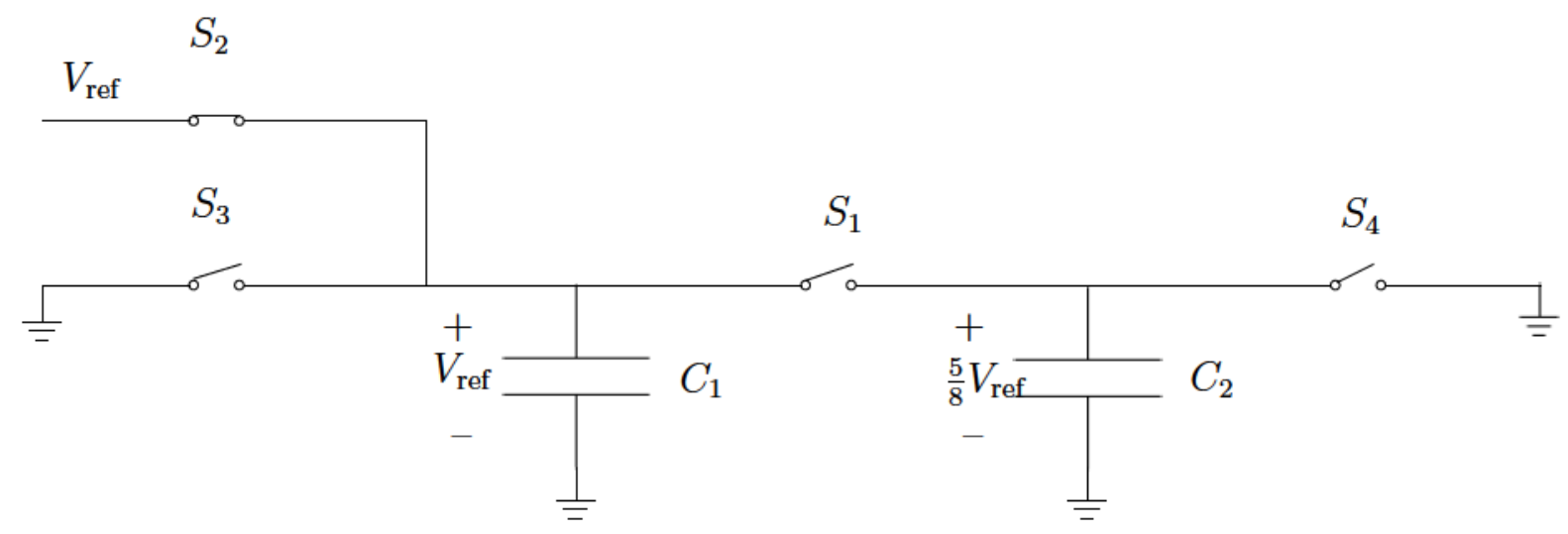

Figure B.11: Serial DAC schematic diagram showing the circuit configuration when capacitor $C_{1}$ is connected to $V_{\text {ref. }}$. The voltage across capacitor $C_{2}$ remains $\frac{5}{8} V_{\text {ref }}$ from the previous step. This configuration is the fifth part of the determination of the fourth MSB when the amplitude of the sampled analog signal is slightly greater than $\frac{13}{16} V_{\text {ref }}$.

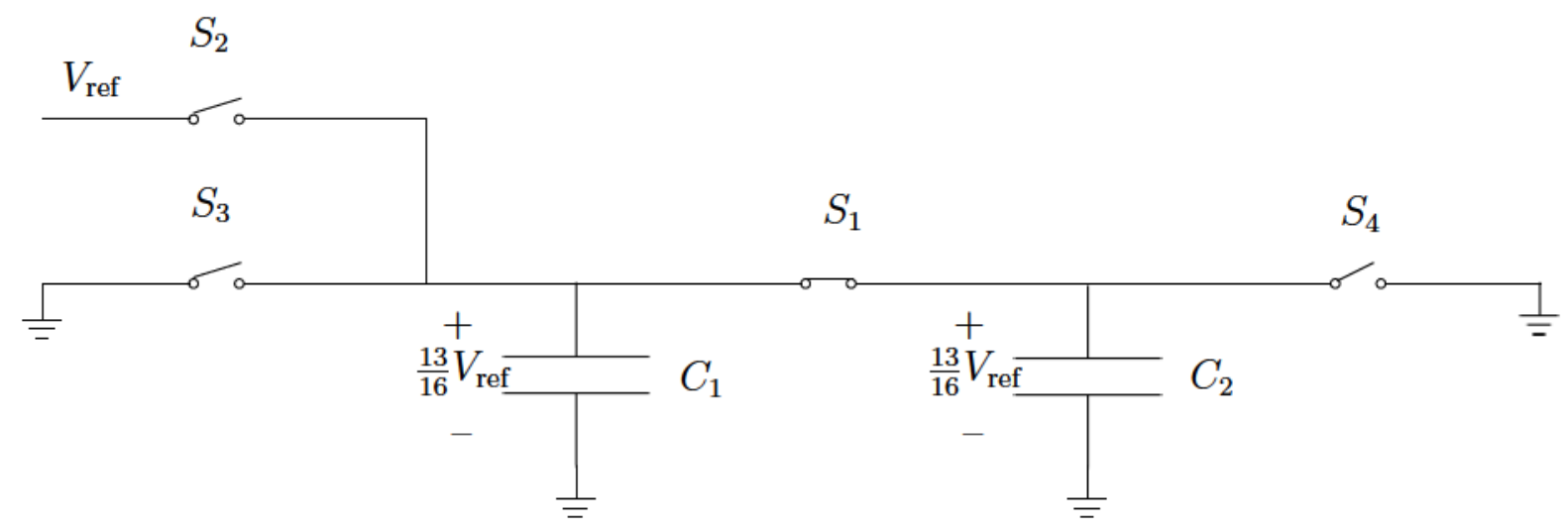

Figure B.12: Serial DAC schematic diagram showing the circuit configuration when capacitors $C_{1}$ and $C_{2}$ are connected in parallel. The voltage across each capacitor is $\frac{13}{16} V_{\text {ref }}$. This configuration is the final part of the determination of the fourth MSB when the amplitude of the sampled analog signal is slightly greater than $\frac{13}{16} V_{\text {ref. }}$. 


\section{Appendix C}

\section{Derivation of SAR Algorithm}


The charge sharing SAR $\mathrm{ADC}$ uses charge recycling to generate the voltages required to convert an analog signal into its digital equivalent. What this means is that the charge sharing SAR ADC will use previously generated voltages, that are still held by capacitors in its capacitor array, to generate the voltage the ADC is currently generating. An example of this is shown in 3.2 .4 .

3.2.4 shows how the charge sharing $\mathrm{SAR}$ ADC generates $\frac{3}{8} V_{\text {ref }}$ using voltages that were generated by this ADC as it generated $\frac{1}{2} V_{\text {ref }}$ and $\frac{1}{4} V_{\text {ref. }} V_{\text {ref }}$ equals $1 \mathrm{~V}$ so the capacitors in the capacitor array are charged to $1 \mathrm{~V}, 0 \mathrm{~V}, \frac{1}{2} \mathrm{~V}$, and $\frac{1}{4} \mathrm{~V}$. To generate $\frac{3}{8} V_{\text {ref }}$, the capacitor array connects three capacitors charged to $\frac{1}{4} \mathrm{~V}$ and one capacitor charged to $0 \mathrm{~V}$. This is how the charge sharing SAR ADC performs charge recycling.

In order to develop an algorithm to implement the charge sharing SAR ADC] SAR logic, Perl code was written to generate all possible combinations of voltages stored across the capacitor array's capacitors for each voltage that the charge sharing SAR ADC generates as it traverses its decision tree. The Perl code is in Appendix D. An example of a decision tree is shown in Figure 2.3 .

The output of the Perl code from Appendix D is a CSV file for each voltage the charge sharing SAR ADC generates (i.e. a CSV file for each voltage in its decision tree, refer to Figure 2.3 for an example of these voltages). The charge sharing SAR ADC has the ability to generate 255 uniquely different voltages. Therefore, the Perl code generated 255 CSV files. These files were read into Excel in order to analyze their data. By looking at the solutions generated for each voltage, the correct sequence of solutions that implemented the voltage required at each node of each path through the decision tree was obtained. The data generated by the Perl code for $\frac{3}{8} V_{\text {ref }}$ is shown in Table C.1.

The data generated by the Perl code for the $\frac{1}{4} V_{\text {ref }}$ and $\frac{1}{2} V_{\text {ref }}$ voltages was consulted to determine which solution to use from Table C.1 to generate $\frac{3}{8} V_{\text {ref. }}$ The data generated by the Perl code for $\frac{1}{4} V_{\text {ref }}$ is shown in Table C.2. The data generated for $\frac{1}{2} V_{\text {ref }}$ is shown in Table C.3. Referring to the solutions for $\frac{1}{4} V_{\text {ref }}$ and $\frac{1}{2} V_{\text {ref }}$ is necessary because the voltages generated for these voltages will be recycled as the ADC generates $\frac{3}{8} V_{\text {ref. }}$.

The generation of $\frac{1}{2} V_{\text {ref }}$ is a special case in that this voltage is created by connecting 


\begin{tabular}{|c|c|c|c|c|}
\hline$\frac{1}{4} \mathrm{~V}$ & $\frac{1}{2} \mathrm{~V}$ & $1 \mathrm{~V}$ & $0 \mathrm{~V}$ & Array Voltage \\
\hline 1 & 1 & 0 & 0 & 0.375 \\
\hline 2 & 2 & 0 & 0 & 0.375 \\
\hline 3 & 3 & 0 & 0 & 0.375 \\
\hline 5 & 0 & 1 & 0 & 0.375 \\
\hline 0 & 3 & 0 & 1 & 0.375 \\
\hline 1 & 4 & 0 & 1 & 0.375 \\
\hline 2 & 0 & 1 & 1 & 0.375 \\
\hline 3 & 1 & 1 & 1 & 0.375 \\
\hline 0 & 1 & 1 & 2 & 0.375 \\
\hline 1 & 2 & 1 & 2 & 0.375 \\
\hline 1 & 0 & 2 & 3 & 0.375 \\
\hline
\end{tabular}

Table C.1: All possible charge sharing SAR ADC's capacitor array voltages when $\frac{3}{8} V_{\text {ref }}$ generated. For case of 15 capacitors in the array. The data in this table was generated by Appendix D $/$ s Perl code.

\begin{tabular}{|c|c|c|c|}
\hline$\frac{1}{2} \mathrm{~V}$ & $1 \mathrm{~V}$ & $0 \mathrm{~V}$ & Array Voltage \\
\hline 1 & 0 & 1 & 0.25 \\
\hline 2 & 0 & 2 & 0.25 \\
\hline 3 & 0 & 3 & 0.25 \\
\hline 0 & 1 & 3 & 0.25 \\
\hline 1 & 1 & 4 & 0.25 \\
\hline
\end{tabular}

Table C.2: All possible charge sharing SAR ADC's capacitor array voltages when $\frac{1}{4} V_{\text {ref }}$ generated. For case of 15 capacitors in the array. The data in this table was generated by Appendix $\mathrm{D} / \mathrm{s}$ Perl code.

\begin{tabular}{|c|c|c|}
\hline $1 \mathrm{~V}$ & $0 \mathrm{~V}$ & Array Voltage \\
\hline 1 & 1 & 0.5 \\
\hline 2 & 2 & 0.5 \\
\hline 3 & 3 & 0.5 \\
\hline
\end{tabular}

Table C.3: All possible charge sharing SAR ADC's capacitor array voltages when $\frac{1}{2} V_{\text {ref }}$ generated. For case of 15 capacitors in the array. The data in this table was generated by Appendix $[\mathrm{D} / \mathrm{s}$ Perl code. 
only capacitors charged to $1 \mathrm{~V}$ and discharged $(0 \mathrm{~V})$. This case is special because some of the voltages generated by the $\mathrm{ADC}$ as it reaches deeper into its decision tree require $1 \mathrm{~V}$ and $0 \mathrm{~V}$. Therefore, looking to all voltages requiring either $1 \mathrm{~V}$ or $0 \mathrm{~V}$ was necessary to determine the number of capacitors that must remain at $1 \mathrm{~V}$ and the number of capacitors that must remain at $0 \mathrm{~V}$ after $\frac{1}{2} V_{\text {ref }}$ is generated. The more capacitors charged to $1 \mathrm{~V}$ and $0 \mathrm{~V}$ as the $\mathrm{ADC}$ traverses deeper into its decision tree means fewer clock cycles are required to converge on a solution. The reason for this is that if the ADC requires $1 \mathrm{~V}$ or $0 \mathrm{~V}$ to generate a specific voltage and none of the capacitors in the array have either $1 \mathrm{~V}$ or $0 \mathrm{~V}$ across it, an additional single clock cycle is required to either charge or discharge the required number of capacitors in the array. Additional clock cycles increase the ADC.s conversion time and decreases the number of times a input signal can be sampled.

Pseudo code for how the charge sharing SAR ADC generates $\frac{3}{8} V_{\text {ref: }}$ :

$1 C_{0}, C_{1}, C_{2}, C_{3}, C_{4}, C_{5}, C_{6}=1 \mathrm{~V} \%$ Charge these capacitors to $1 \mathrm{~V}$.

$2 C_{7}, C_{8}, C_{9}, C_{10}, C_{11}, C_{12}, C_{13}, C_{14}=0 \mathrm{~V} \%$ Discharge these capacitors.

3 Connect capacitors $C_{0}, C_{1}, C_{2}, C_{7}, C_{8}, C_{9}$ in parallel (and also not connected to a supply voltage $(1 \mathrm{~V})$ nor ground) to generate $\frac{1}{2} V_{\text {ref }}$.

4 Connect capacitors $C_{7}, C_{8}, C_{9}, C_{10}, C_{11}, C_{12}$ in parallel (and also not connected to a supply voltage $(1 \mathrm{~V})$ nor ground) to generate $\frac{1}{4} V_{\text {ref }}$.

5 Connect capacitors $C_{6}, C_{7}, C_{8}, C_{13}$ in parallel (and also not connected to a supply voltage (1V) nor ground) to generate $\frac{3}{8} V_{\text {ref }}$.

The 255 CSV files were generated many times. The number of capacitors in the charge sharing SAR ADC 3 capacitor array directly affect the solutions generated by the Perl code. Therefore, an iterative process of "how many capacitors in the array are needed" caused the generation of many sets of CSV files. The deciding factor for how many capacitors was driven by balancing the need to have the fewest number of capacitors possible with a number of capacitors necessary to generate all required voltages. It 
was determined that 15 capacitors were the minimum number required in the array to correctly generate all of the required voltages as the algorithm traverses the decision tree.

The final code that controls the connection of the capacitors in the capacitor array is a case statement implemented in the Verilog hardware description language. A detailed flowchart of the case statement is in Appendix ??. 


\section{Appendix D}

Capacitor Array Voltage Generation

Code 
$\# ! c: \backslash$ strawberry\perl\bin\perl

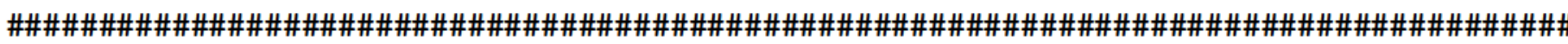

\# Step 1: Have user enter the voltages and number of capacitors.

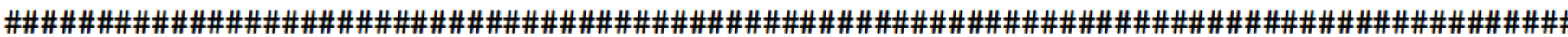

print "Please enter the voltage values (separated by spaces):";

chomp ( inputvoltages $=\langle$ STDIN $\rangle$ );

\$inputvoltages $=\sim \mathrm{s} /(\wedge \mathrm{s}+) \mid(\backslash \mathrm{s}+\$) / / \mathrm{g} ; \quad$ \# Remove any leading or trailing spaces.

@voltages $=\operatorname{split}(\bigwedge \mathrm{s}+/$, \$inputvoltages $) ;$

\$data_size = Qdata; \# Get the size of the array.

print "\nEnter the number of capacitors: ";

chomp (\$input_capacitors $=\langle$ STDIN $\rangle$ );

print "\nEnter the target voltage: ";

chomp (\$target_voltage $=\langle$ STDIN $\rangle$ );

\$number_voltages = @voltages;

print "input_capacitors: \$input_capacitors $\backslash n \backslash n "$;

for \$voltage (0.. (\$number_voltages -1$)$ ) \{

print "Entered data value \$voltage = \$voltages[\$voltage] $\backslash n$ ";

\}

print "\nNumber_voltages: \$number_voltages $\backslash \mathrm{n}$ ";

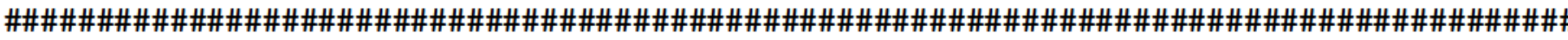

\# Step 2: Initalize column_value array.

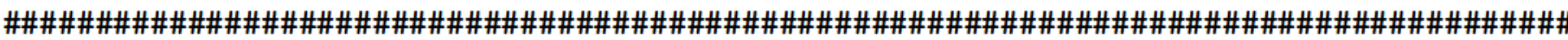




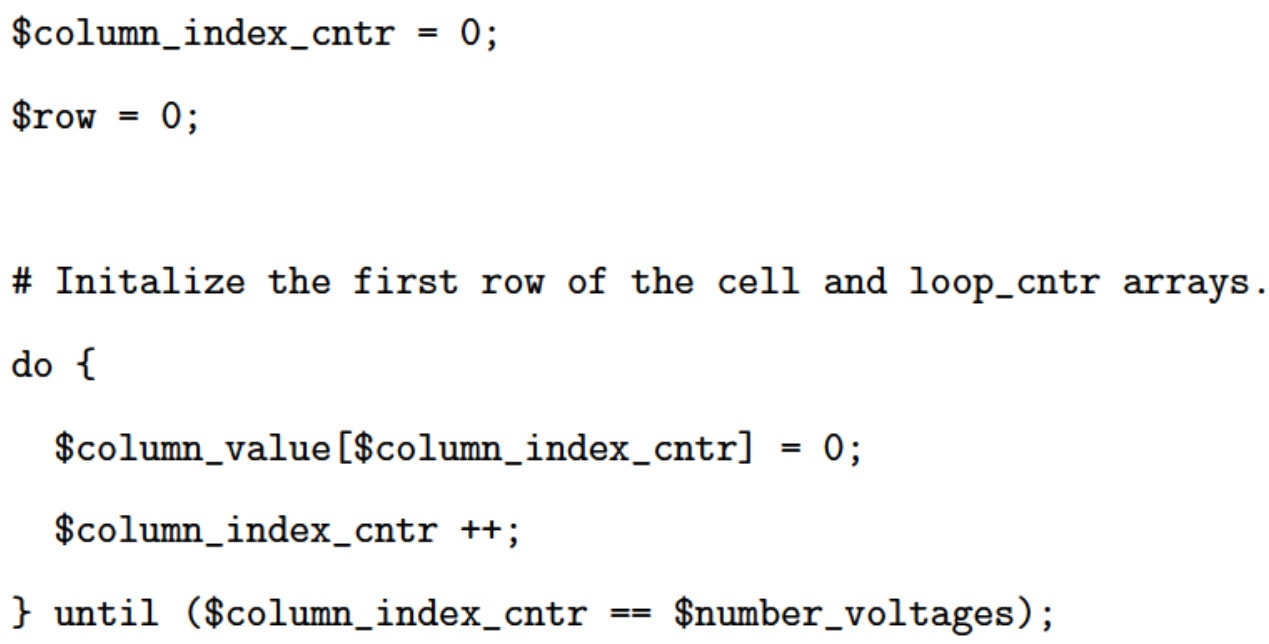


do \{

\$capacitor [\$row] [\$write_column_cntr] = \$column_value [\$write_column_cntr];

\$write_column_cntr ++;

\} until (\$write_column_cntr == \$number_voltages);

\# Move on to the next row.

$\$$ column_cntr $=0$;

\$column_value [\$column_cntr] ++;

\# End when all of the elements on the column_value array equal \$input_capacitors. if (\$column_value[\$column_cntr] >= \$input_capacitors +1$)\{$

do \{

\$column_value [\$column_cntr $]=0$;

\$column_cntr ++;

\$column_value [\$column_cntr] ++;

\} until (\$column_value $[\$$ column_cntr $]<($ input_capacitors +1$)$ );

\} else \{

$\$$ sum $=0 ;$

$\$$ column_check_cntr $=0$;

\$element_check_cntr $=0$;

\$element_input_capacitor $=0$;

\# Check if each element is \$input_capacitors. Quit when they are. do \{ 


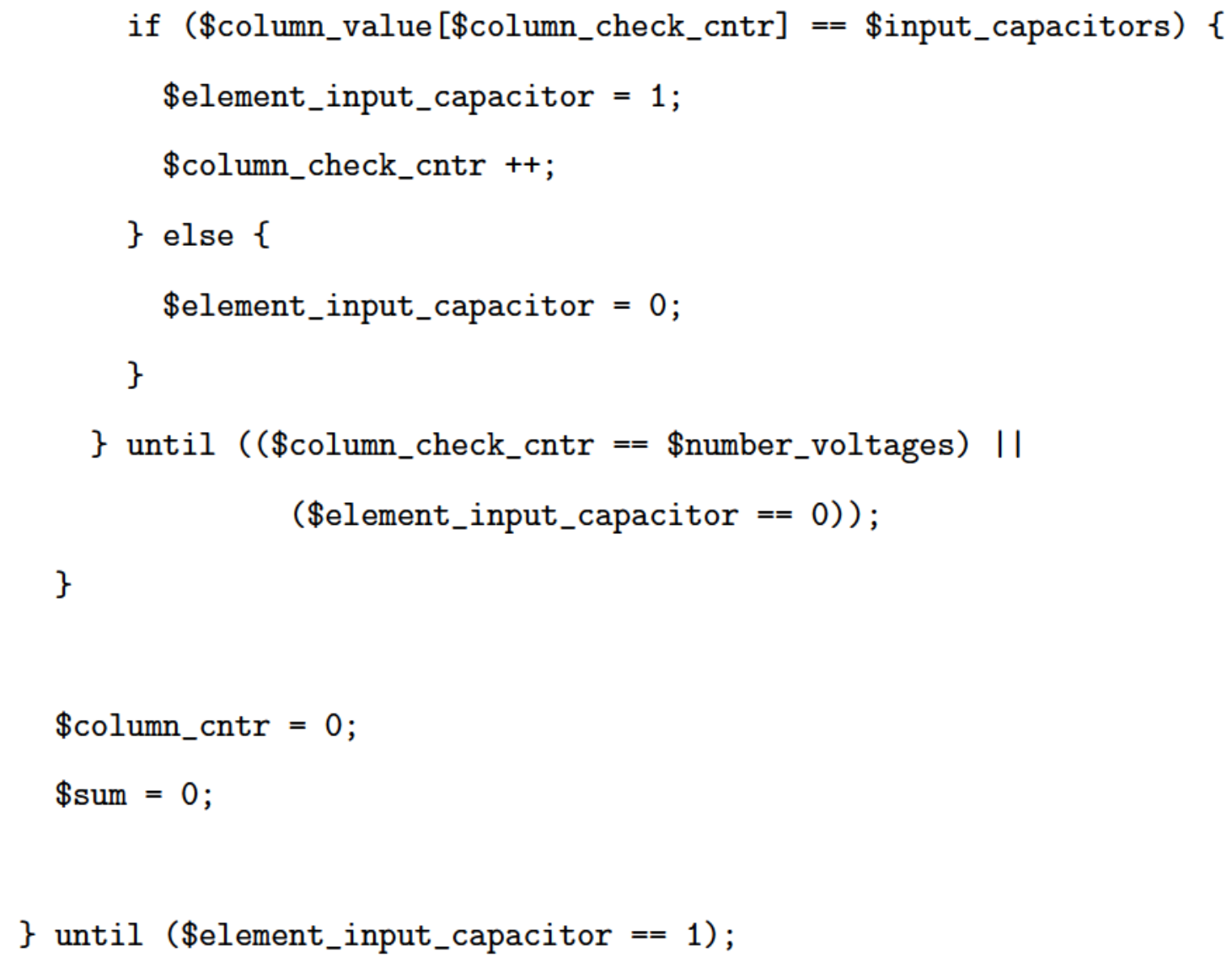


do \{

\# Calculate the output voltage of the capacitor array for the current row of \# capacitor value in the array "capacitor".

do \{

\$sum_volt_cap $=\$$ sum_volt_cap $+(\$$ voltages $[\$$ column_cntr $] *$

\$capacitor [\$row_cntr] [\$column_cntr]);

\$sum_cap $=$ \$sum_cap $+($ capacitor $[$ \$row_cntr $][\$$ column_cntr $]) ;$

\$column_cntr ++;

\} until (\$column_cntr $==$ \$number_voltages);

if ( $\$$ sum_cap $>0)\{$

\$array_voltage[\$row_cntr] = \$sum_volt_cap / \$sum_cap;

\} else \{

\$array_voltage[\$row_cntr] $=0$;

\}

if (\$array_voltage[\$row_cntr] == \$target_voltage) \{

\# Store the row of capacitor values and the array voltage when the larger array \# voltage is calculated.

do \{

print OUTPUT "\$capacitor[\$row_cntr] [\$goal_column_cntr], ";

\$goal_column_cntr ++;

\} until (\$goal_column_cntr == \$number_voltages);

print OUTPUT " I sum_volt_cap \$sum_volt_cap"; 
print OUTPUT " I sum_cap \$sum_cap";

print OUTPUT " I array_voltage \$array_voltage[\$row_cntr] \n";

\}

\$row_cntr ++;

\# Initalize column counter for next row

$\$$ column_cntr $=0$;

\$goal_column_cntr $=0$;

\$sum_volt_cap $=0$;

\$sum_cap $=0$;

\} until (\$row_cntr $==(\$$ row +1$))$; 


\section{Appendix E}

\section{Derivation of Charge Sharing}

\section{Equations}


Using Kirchoff's Voltage Law, the sum of voltages for the capacitors shown in Figure 3.4 is described by Equation E.1.

$$
0=-V_{\mathrm{C} 0}^{\prime}-V_{\mathrm{C} 1}^{\prime}-V_{\mathrm{C} 2}^{\prime}-V_{\mathrm{C} 7}^{\prime}-V_{\mathrm{C} 8}^{\prime}-V_{\mathrm{C} 9}^{\prime}
$$

The equation that defines a capacitor's capacitance is described by Equation E.2,

$$
Q=C V
$$

where, $\mathrm{Q}$ is the charge stored on the capacitor, $\mathrm{C}$ is the capacitor's capacitance, and $\mathrm{V}$ is the voltage across the capacitor.

Substituting Equation E.2 into Equation E.1 results in Equation E.3,

$$
0=\frac{-Q_{\mathrm{C} 0}^{\prime}}{C 1}-\frac{Q_{\mathrm{C} 1}^{\prime}}{C 1}-\frac{Q_{\mathrm{C} 2}^{\prime}}{C 2}-\frac{Q_{\mathrm{C} 7}^{\prime}}{C 7}-\frac{Q_{\mathrm{C} 8}^{\prime}}{C 8}-\frac{Q_{\mathrm{C} 9}^{\prime}}{C 9}
$$

Let $\mathrm{q}$ be the charge lost from a capacitor as it connected to another capacitor. This equation that describes this is Equation E.4.

$$
Q^{\prime}=Q-q
$$

Substituting Equation E.4 into Equation E.3 yields Equation E.5.

$$
0=\frac{-Q_{\mathrm{C} 0}+q}{C 0}-\frac{Q_{\mathrm{C} 1}+q}{C 1}-\frac{Q_{\mathrm{C} 2}+q}{C 2}-\frac{Q_{\mathrm{C} 7}+q}{C 7}-\frac{Q_{\mathrm{C} 8}+q}{C 8}-\frac{Q_{\mathrm{C} 9}+q}{C 9}
$$


Solving Equation E.5 for q results in Equation E.6.

$$
\begin{aligned}
& Q_{\mathrm{C} 0} C_{1} C_{2} C_{7} C_{8} C_{9}+Q_{\mathrm{C} 1} C_{0} C_{2} C_{7} C_{8} C_{9}+Q_{\mathrm{C} 2} C_{0} C_{1} C_{7} C_{8} C_{9} \\
& q=\frac{+Q_{\mathrm{C} 7} C_{0} C_{1} C_{2} C_{8} C_{9}+Q_{\mathrm{C} 8} C_{0} C_{1} C_{2} C_{7} C_{9}+Q_{\mathrm{C} 9} C_{0} C_{1} C_{2} C_{7} C_{8}}{C_{1} C_{2} C_{7} C_{8} C_{9}+C_{0} C_{2} C_{7} C_{8} C_{9}+C_{0} C_{1} C_{7} C_{8} C_{9}} \\
& +C_{0} C_{1} C_{2} C_{8} C_{9}+C_{0} C_{1} C_{2} C_{7} C_{9}+C_{0} C_{1} C_{2} C_{7} C_{8}
\end{aligned}
$$

Substituting Equation E.4 into Equation E.6 results in Equation E.7.

$$
\begin{aligned}
q= & \frac{C_{0} C_{1} C_{2} C_{7} C_{8} C_{9}\left(V_{0}+V_{1}+V_{2}+V_{7}+V_{8}+V_{9}\right)}{C_{1} C_{2} C_{7} C_{8} C_{9}+C_{0} C_{2} C_{7} C_{8} C_{9}+C_{0} C_{1} C_{7} C_{8} C_{9}} \\
& +C_{0} C_{1} C_{2} C_{8} C_{9}+C_{0} C_{1} C_{2} C_{7} C_{9}+C_{0} C_{1} C_{2} C_{7} C_{8}
\end{aligned}
$$

Combining Equation E.2 with Equation E.4 results in Equation E.8

$$
V_{\mathrm{x}}^{\prime}=\frac{Q_{\mathrm{x}}^{\prime}}{C_{\mathrm{x}}}=\frac{Q_{\mathrm{x}}-q}{C_{\mathrm{x}}}=\frac{C_{\mathrm{x}} V_{\mathrm{x}}-q}{C_{\mathrm{x}}}=V_{\mathrm{x}}-\frac{q}{C_{\mathrm{x}}}
$$

where, $\mathrm{x}$ is $1,2,3,7,8,9$ since this describes the change in voltage and charge as capacitors $C_{1}, C_{2}, C_{3}, C_{7}, C_{8}$, and $C_{9}$ are connected in parallel.

Substituting Equation E.8 into Equation E.7 yields the following 6 equations Equation E.9, Equation E.10, Equation E.11, Equation E.12, Equation E.13, Equation E.14.

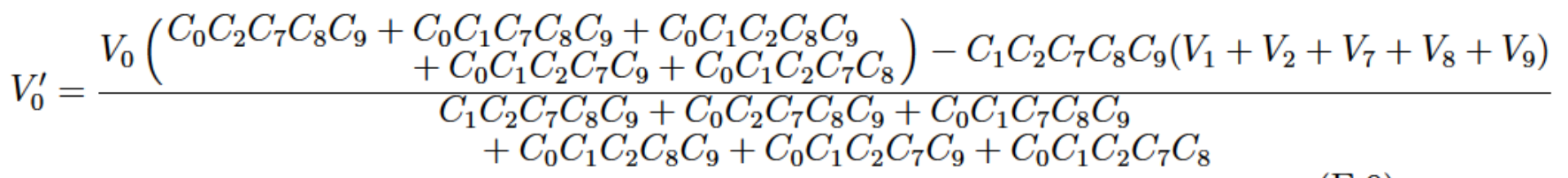

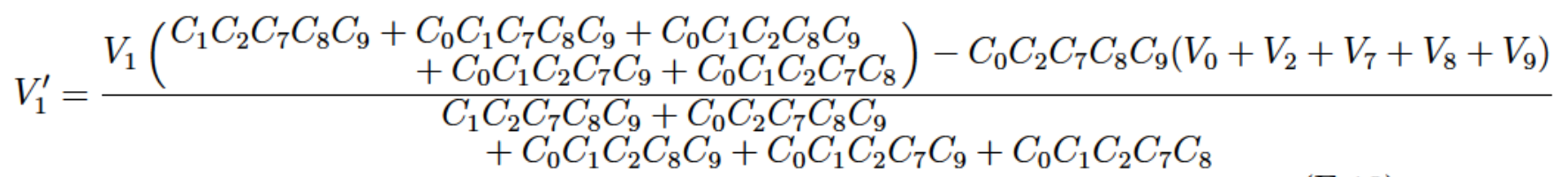




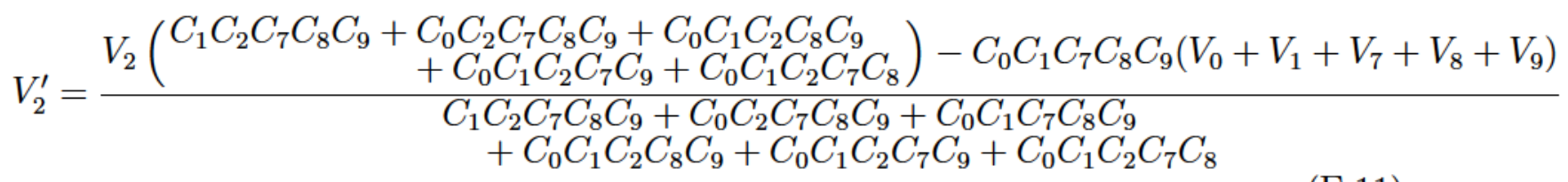

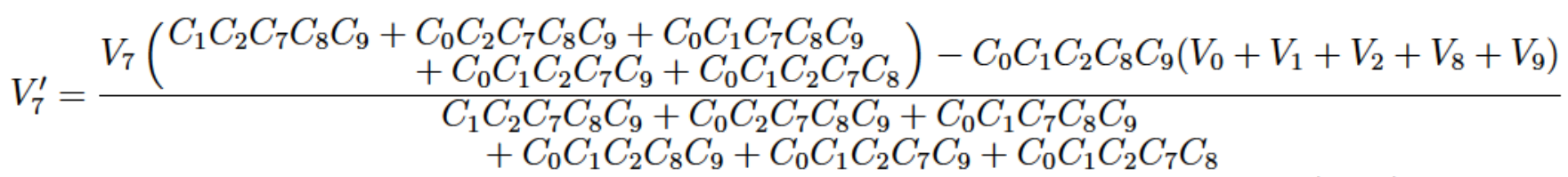

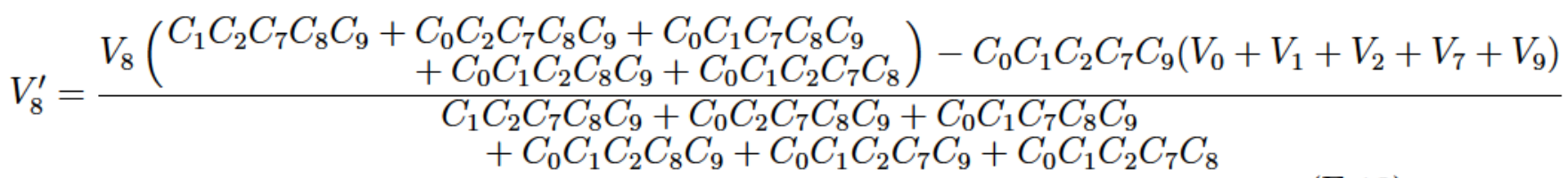

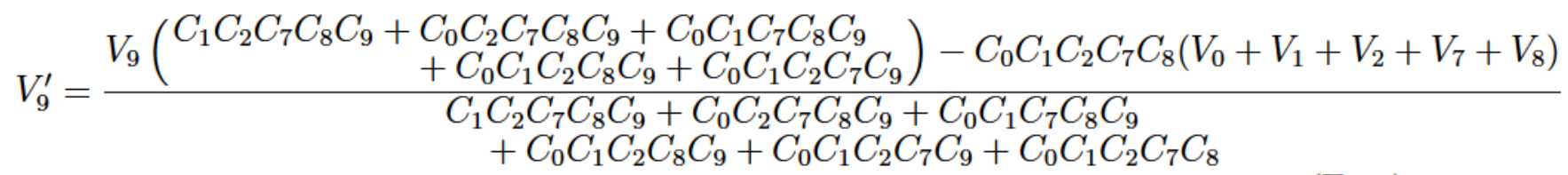

Since all the capacitors in the charge Sharing SAR ADC's capacitor array have the same capacitance, Equation E.9, Equation E.10, Equation E.11, Equation E.12, Equation E.13, and Equation E.14 reduce to Equation E.15, Equation E.16, Equation E.17, Equation E.18, Equation E.19, and Equation E.20 respectively.

$$
V_{0}^{\prime}=\frac{V_{0}(5)-\left(V_{1}+V_{2}+V_{7}+V_{8}+V_{9}\right)}{6}
$$


$V_{1}^{\prime}=\frac{V_{1}(5)-\left(V_{0}+V_{2}+V_{7}+V_{8}+V_{9}\right)}{6}$

$V_{2}^{\prime}=\frac{V_{2}(5)-\left(V_{0}+V_{1}+V_{7}+V_{8}+V_{9}\right)}{6}$

$V_{7}^{\prime}=\frac{V_{7}(5)-\left(V_{0}+V_{1}+V_{2}+V_{8}+V_{9}\right)}{6}$

$V_{8}^{\prime}=\frac{V_{8}(5)-\left(V_{0}+V_{1}+V_{2}+V_{7}+V_{9}\right)}{6}$

$$
V_{9}^{\prime}=\frac{V_{9}(5)-\left(V_{0}+V_{1}+V_{2}+V_{7}+V_{8}\right)}{6}
$$




\section{Appendix F}

Factors that degrade $\mathrm{S} / \mathrm{H}$

performance 


\section{F.1 Charge injection}

Charge injection occurs when a transistor turns off and the charge used to form its channel flows out of the transistor's source and drain terminals. This charge distorts the sampled signal by either increasing (an NMOS transistor's channel charge is electrons which increase the sampled charge stored by the sampling capacitor) or decreasing (a PMOS transistor's channel charge is holes which decrease the sampled charge stored by the sampling capacitor) the voltage stored on capacitors $\mathrm{C}_{1}$ and $\mathrm{C}_{2}$.

As [31] and 32] describe, if the voltage applied to a transistor's gate is slowly reduced, its channel charge can be absorbed by the substrate. If the voltage applied to the transistor's gate is reduced too quickly, the substrate can't absorb all of the channel charge and the un-absorbed charge flows out of the transistor's source and drain terminals. Therefore, it is desirable to ensure that the clock signal applied to the $\mathrm{S} / \mathrm{H}$ s switches have as long a rise and fall time as possible to reduce charge injection.

\section{F.2 Clock feedthrough}

Clock feedthrough error is due to capacitive coupling to the $\mathrm{S} / \mathrm{H}$ capacitor $\mathrm{C}_{\mathrm{L}}$ from the overlap capacitor $\mathrm{C}_{\mathrm{gd}}$ and the gate capacitor $\mathrm{C}_{\mathrm{ox}}[13]$. The amount by which the voltage stored on the $\mathrm{S} / \mathrm{H}$ 's capacitor, $\mathrm{C}_{\mathrm{L}}$, is modified is determined by the difference between the coupled charge and the charge injected by the transistor current.

Figure F.1 shows the currents that flow in a CMOS transistor.

Figure F.1 shows two coupling currents, $\mathrm{I}_{\mathrm{ox}}$ due to the oxide capacitance, and $\mathrm{I}_{\mathrm{gd}}$ due to the parasitic gate-drain overlap capacitor. The coupling currents $\mathrm{I}_{\mathrm{ox}}$ and $\mathrm{I}_{\mathrm{gd}}$ distort the transistor drain current $\mathrm{I}_{\mathrm{D}}$.

Figure F.2 shows the currents that flow in the CMOS transmission gate. The capacitors, $\mathrm{C}_{\mathrm{ox}}$ and $\mathrm{C}_{\mathrm{gd}}$, couple currents $\mathrm{I}_{\mathrm{oxn}}$ and $\mathrm{I}_{\mathrm{gdn}}$ from the nfet and $\mathrm{I}_{\mathrm{oxp}}$ and $\mathrm{I}_{\mathrm{gdp}}$ from the pfet into the load capacitor, modifying the voltage stored on $\mathrm{C}_{\mathrm{L}}$ as the transmission gate turns off.

Ideally, when a transmission gate is used as a switch, the coupling currents through 


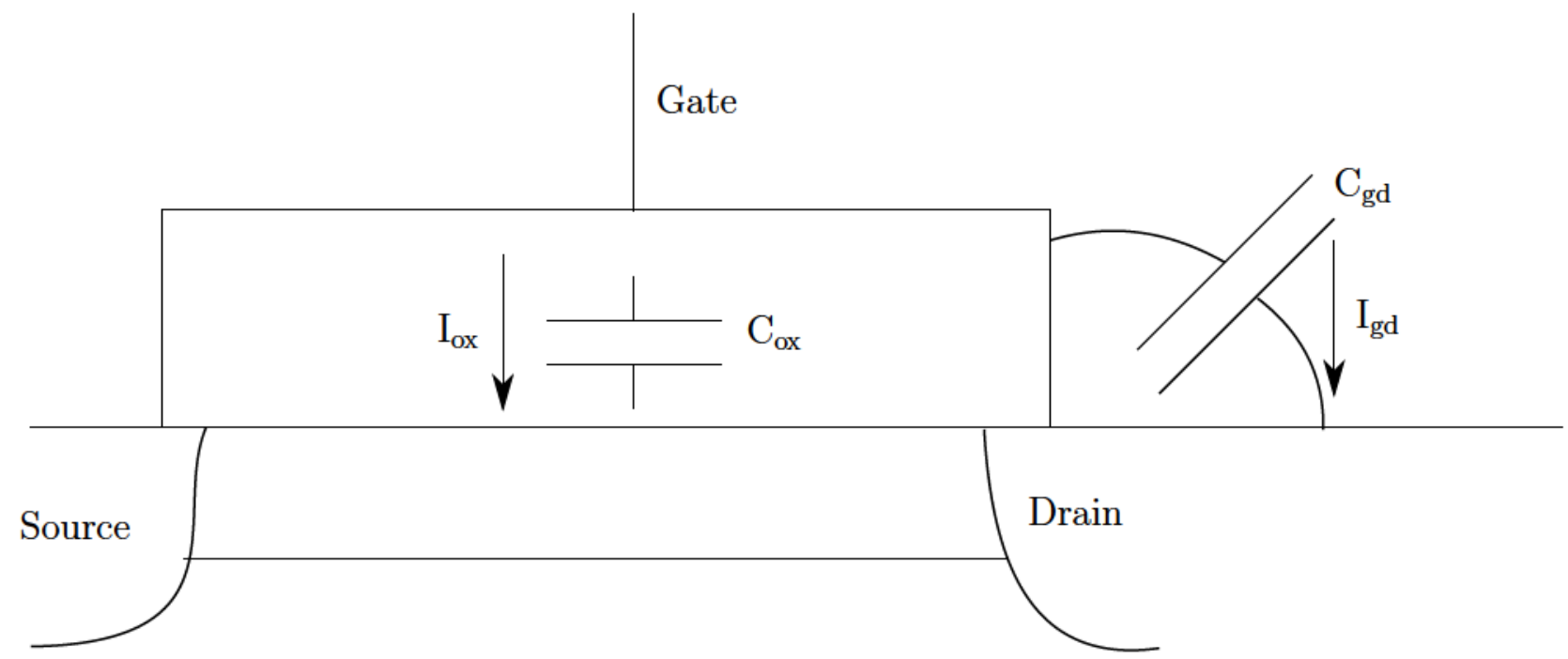

Figure F.1: CMOS transistor drain current, $\mathrm{I}_{\mathrm{d}}$, and coupling currents, $\mathrm{I}_{\mathrm{ox}}$ and $\mathrm{I}_{\mathrm{gd}}$.

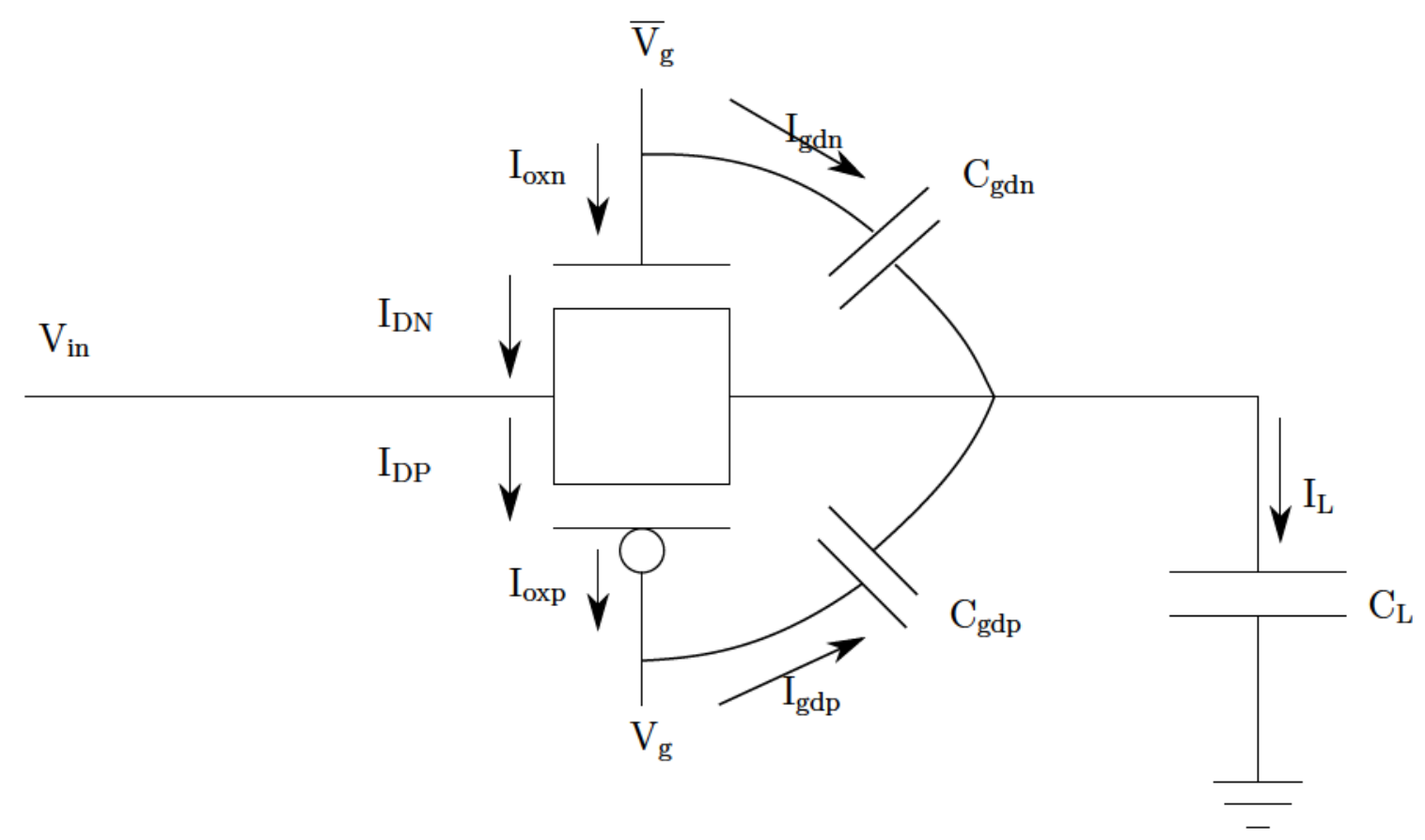

Figure F.2: CMOS transmission gate drain currents $\mathrm{I}_{\mathrm{DN}}$ and $\mathrm{I}_{\mathrm{DP}}$, and coupling currents, $\mathrm{I}_{\mathrm{oxn}}, \mathrm{I}_{\mathrm{oxp}}$, $\mathrm{I}_{\mathrm{gdn}}$, and $\mathrm{I}_{\mathrm{gdp}}$.

the nfet and pfet are the same magnitude and cancel each other. The reason why the currents should cancel is due to the fact that the current from the nfet flows in the opposite direction relative to the pfet current. Unfortunately in practice, these currents aren't the same magnitude and don't completely cancel each other. The first factor affecting the magnitude of these currents is due to manufacturing defects. These defects manifest themselves as a difference in the size of the overlap capacitors, and therefore magnitude of stored charge. The second factor is the skew, or difference in timing of 
the signals applied to, or removed from, the gates of the nfet and pfet transistors. In a simplified explanation, the result of this skew is that the nfet and pfet are turned off at different times, causing the coupling currents to flow at different times with the result that these currents aren't canceled completely.

[13] provides a more sophisticated explanation of how clock skew distorts the charge stored on the load capacitance as the clock applied to a transmission gate is removed. Figure F.3 shows the gate voltages required to turn on transmission gate nfet and pfet transistors. The pfet is turned on when $V_{\text {in }}$ is greater than the black line shown in Figure F.3 and the nfet is turned on when $\mathrm{V}_{\text {in }}$ is less than the red line shown in Figure F.3. This figure was generated assuming that both transistors have the same width, length, and equal, but opposite threshold voltages.

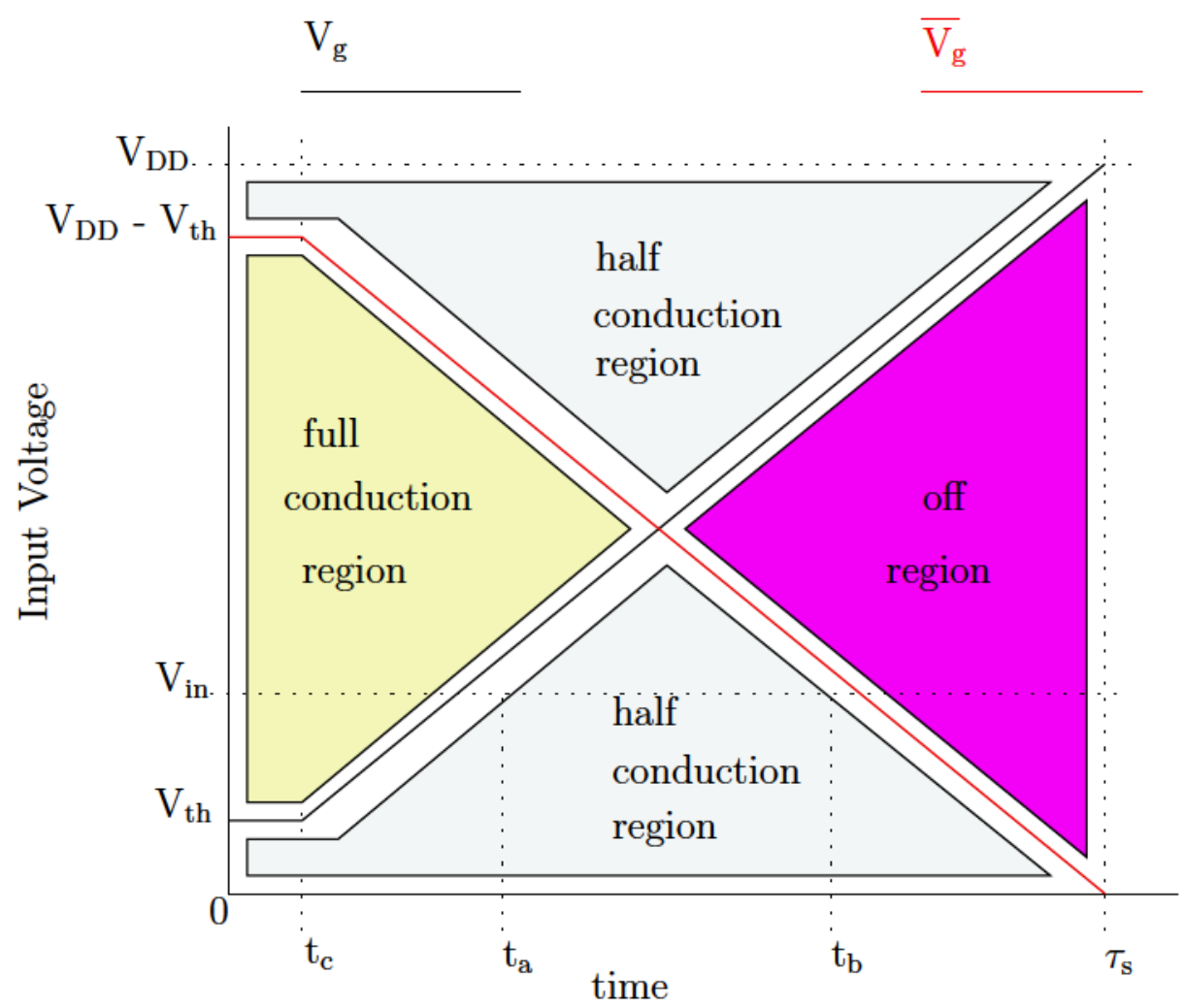

Figure F.3: CMOS transmission gate operating regions: full conduction, half conduction, and off. The pfet is on whenever its gate voltage is greater than $V_{\text {th }}$ (i.e. its gate voltage is greater than the black line). The nfet is on whenever its gate voltage is less than $V_{D D}-V_{\text {th }}$ (i.e. its gate voltage is below the red line). $\tau_{\mathrm{s}}$ is the fall time of the clock.

Figure F.3 shows three operating regions described in subsections F.3, F.3.1 and F.3.2. 


\section{F.3 Conduction}

In this region both the nfet and pfet transistors are turned on. The coupling currents due to the gate and also the source to drain overlap capacitances modify, or distort, the drain current through transmission gate, causing an incorrect voltage to be stored across the load capacitor. The current flowing into the load capacitor is described to the first order in this region by Equation F.1.

$$
I_{\mathrm{L}}=I_{\mathrm{gdn}}+I_{\mathrm{gdp}}-\left(I_{\mathrm{DN}}-I_{\mathrm{oxn}}\right)+\left(I_{\mathrm{DP}}-I_{\mathrm{oxp}}\right)
$$

However, the amount of distortion is a function of the input voltage, transition time of the gate voltage, load capacitance, and size of the transistors. Therefore, a more complete equation to calculate the amount of distortion is obtained by substituting Equation F.2, Equation F.3, Equation F.4, and Equation F.5 into Equation F.6,

$$
C_{\text {Leff }}=C_{L}+C_{g d n}+C_{g d p}
$$

where, $\mathrm{C}_{L}$ is the load capacitor, $\mathrm{C}_{g d n}$ is the nfet gate to drain capacitance, and $\mathrm{C}_{g d p}$ is the pfet gate to drain capacitance.

$$
k_{n}=\mu_{n} C_{o x N}\left(\frac{W}{L}\right)_{N}
$$

where, $\mu_{n}$ is electron mobility, $\mathrm{C}_{o x N}$ is the nfet oxide capacitance, and $\left(\frac{W}{L}\right)_{N}$ is the nfet width to length ratio.

$$
k_{p}=-\left|\mu_{p}\right| C_{o x P}\left(\frac{W}{L}\right)_{P}
$$


where, $\mu_{p}$ is the hole mobility, $\mathrm{C}_{o x P}$ is the pfet oxide capacitance, and $\left(\frac{W}{L}\right)_{P}$ is the pfet width to length ratio.

$$
A_{1}=\frac{k_{n} V_{d d}-\left(k_{n}+\left|k_{p}\right|\right) V_{i n}-k_{n} V_{T N}+\left|k_{p}\right|\left|V_{T P}\right|}{k_{n}-\left|k_{p}\right|}
$$

$$
\begin{aligned}
& V_{e 1}(t)=-\left[\sqrt{\frac{\pi V_{d d} C_{L e f f}}{2 \tau_{s}\left(k_{n}-\left|k_{p}\right|\right)}}\right]\left(\frac{2 C_{g d n}-2 C_{g d p}+C_{o x N}-C_{o x P}}{2 C_{L e f f}}\right) \exp \left\{\frac{\left(k_{n}-\left|k_{p}\right|\right) V_{d d}}{2 \tau_{s} C_{L e f f}}\left(t-\frac{A_{1} \tau_{s}}{V_{d d}}\right)^{2}\right\} \\
& \left\{\operatorname{erf}\left[\sqrt{\frac{\tau_{s}\left(k_{n}-\left|k_{p}\right|\right)}{2 V_{d d} C_{L e f f}} A_{1}}\right]-\operatorname{erf}\left[\sqrt{\frac{\tau_{s}\left(k_{n}-\left|k_{p}\right|\right)}{2 V_{d d} C_{L e f f}}}\left(A_{1}-\left(\frac{V_{d d}}{\tau_{s}}\right) t\right)\right]\right\}
\end{aligned}
$$

where, $\mathrm{V}_{d d}$ is the supply voltage and $\tau_{s}$ is the fall time of clock.

From Equation F.6 it is seen that the error is largest when the input voltage is approximately half of the power supply voltage. This is due to the fact that at this voltage, the on resistance of the transmission gate is at it largest point and therefore, the error voltage across the load capacitance is at its largest value.

\section{F.3.1 Half Conduction}

In this region one of the transistors conduct and the other doesn't. This results in a higher error voltage than when the transmission gate operates in the full conduction region. In the full conduction region, both pfet and nfet transistors conduct and therefore, the coupling currents due to the pfet transistor somewhat cancel the coupling currents due to the nfet transistor. Unfortunately, when the transmission gate operates in this half conduction region, only one of the transistors conducts and therefore no opposing coupling current exists to partially cancel the coupling currents flowing from transistor that is being turned off.

To calculate the clock feedthrough error voltage due to a pfet only, substitute Equa- 
tion F.7 and Equation F.8 into Equation F.9, substitute Equation F.10 and Equation F.11 and Equation [F.12 into Equation [F.13 and then substitute Equation F.9] and Equation F.13 into (F.14).

$$
C_{\text {Leff }}=C_{L}+C_{g d n}+C_{g d p}
$$

where, $\mathrm{C}_{L}$ is the load capacitor, $\mathrm{C}_{g d n}$ is the nfet gate to drain capacitance, and $\mathrm{C}_{g d p}$ is the pfet gate to drain capacitance.

$$
k_{p}=-\left|\mu_{p}\right| C_{o x P}\left(\frac{W}{L}\right)_{P}
$$

where, $\mu_{p}$ is the hole mobility, $\mathrm{C}_{o x P}$ is the pfet oxide capacitance, and $\left(\frac{W}{L}\right)_{P}$ is the pfet width to length ratio.

$$
\beta_{P}=-\sqrt{\frac{\pi V_{d d} C_{L e f f}}{2 \tau_{s}\left|k_{P}\right|}}\left(\frac{C_{g d n}-C_{g d p}+\frac{C_{o x P}}{2}}{C_{L e f f}}\right)
$$

where, $\mathrm{V}_{d d}$ is the supply voltage, $\tau_{s}$ is the fall time of clock,

$$
A_{\text {half }_{P}}=\exp \left\{\frac{\left|k_{P}\right| V_{d d}}{2 \tau_{s} C_{\text {Leff }}}\left(\left(t-t_{b}\right)-\left(\frac{V_{\text {in }}-\left|V_{T P}\right|}{V_{d d}}\right) \tau_{s}\right)^{2}\right\}
$$

where, $\mathrm{V}_{T P}$ is the pfet threshold voltage

$$
\begin{gathered}
B_{\text {half }_{P}}=\operatorname{erf}\left[\sqrt{\frac{\left|k_{p}\right| \tau_{s}}{2 V_{d d} C_{\text {Leff }}}}\left(V_{\text {in }}-\left|V_{T P}\right|\right)\right] \\
C_{\text {half }_{P}}=\operatorname{erf}\left[\sqrt{\frac{\left|k_{p}\right| \tau_{s}}{2 V_{d d} C_{\text {Leff }}}}\left(V_{\text {in }}-\left|V_{T P}\right|-\left(\frac{V_{d d}}{\tau_{s}}\right) t\right)\right]
\end{gathered}
$$




$$
f_{P}(t)=A_{\text {half }_{P}}\left\{B_{\text {half }_{P}}-C_{\text {half }_{P}}\right\}
$$

$$
V_{e 2}(t)=-\beta_{P} f_{P}(t)+V_{e 1}\left(t_{b}\right), t_{b}<t<t_{a}
$$

To calculate the clock feedthrough error voltage due to a nfet only, substitute Equation F.15 and Equation F.16 into Equation F.17 and substitute Equation F.18 and Equation F.19 and Equation F.20 into Equation F.21 and then substitute Equation F.17 and Equation F.21 into Equation F.22.

$$
C_{\text {Leff }}=C_{L}+C_{g d n}+C_{g d p}
$$

where, $\mathrm{C}_{L}$ is the load capacitor, $\mathrm{C}_{g d n}$ is the nfet gate to drain capacitance, and $\mathrm{C}_{g d p}$ is the pfet gate to drain capacitance.

$$
k_{n}=-\left|\mu_{n}\right| C_{o x N}\left(\frac{W}{L}\right)_{N}
$$

where, $\mu_{n}$ is the electron mobility, $\mathrm{C}_{o x N}$ is the nfet oxide capacitance, and $\left(\frac{W}{L}\right)_{N}$ is the nfet width to length ratio.

$$
\beta_{N}=-\sqrt{\frac{\pi V_{d d} C_{L e f f}}{2 \tau_{s} k_{n}}}\left(\frac{C_{g d n}-C_{g d p}+\frac{C_{o x N}}{2}}{C_{L e f f}}\right)
$$


where, $\mathrm{V}_{d d}$ is the supply voltage, $\tau_{s}$ is the fall time of clock

$$
\begin{gathered}
A_{\text {half }_{N}}=\exp \left\{\frac{\left|k_{N}\right| V_{d d}}{2 \tau_{s} C_{\text {Leff }}}\left(\left(t-t_{a}\right)-\left(\frac{V_{\text {in }}-\left|V_{T N}\right|}{V_{d d}}\right) \tau_{s}\right)^{2}\right\} \\
B_{\text {half }_{N}}=\operatorname{erf}\left[\sqrt{\frac{\left|k_{n}\right| \tau_{s}}{2 V_{d d} C_{\text {Leff }}}}\left(V_{\text {in }}-V_{T N}\right)\right]
\end{gathered}
$$

where, $\mathrm{V}_{T N}$ is the nfet threshold voltage.

$$
\begin{gathered}
C_{\text {half }}=\operatorname{erf}\left[\sqrt{\frac{\left|k_{n}\right| \tau_{s}}{2 V_{d d} C_{\text {Leff }}}}\left(V_{\text {in }}-V_{T N}-\left(\frac{V_{d d}}{\tau_{s}}\right) t\right)\right] \\
f_{N}(t)=A_{\text {half } f_{N}}\left\{B_{\text {half } f_{N}}-C_{\text {half }}\right\} \\
V_{e 2}(t)=-\beta_{N} f_{N}(t)+V_{e 1}\left(t_{a}\right), t_{a}<t<t_{b}
\end{gathered}
$$

\section{F.3.2 Off Region}

This is also known as the sub threshold or the cutoff region. In this region, the coupling currents are only due to the gate overlap capacitances $\mathrm{C}_{g d N}$ and $\mathrm{C}_{g d P}$. Both transistors are turned off. Since there is no channel, and the gate resistance is so high, the coupling currents $\mathrm{I}_{o x N}$ and $\mathrm{I}_{o x P}$ are negligible. 


\section{F.4 Sampling Jitter}

Sampling jitter occurs when the $\mathrm{S} / \mathrm{H}$ circuit samples the input analog signal at an incorrect time. This jitter usually manifests itself such that the sampling period isn't consistent and therefore the analog signal isn't sampled at the same point of the sampling clock period. This can lead to incorrectly sampling the analog signal, aliasing it so that the sampled signal is different than the input analog signal.

\section{F.5 Voltage Droop}

Voltage droop is the amount of voltage discharged from the storage capacitor while the $\mathrm{S} / \mathrm{H}$ circuit is in hold mode. The amount of voltage droop, or droop rate, is a function of the leakage currents drawn by the parasitics, for example through the substrate [33].

\section{F.6 Resistance Variation During Track Mode}

The transmission gate switches used by the $\mathrm{S} / \mathrm{H}$ apply the sampled signal to the $\mathrm{S} / \mathrm{H}$ s storage capacitor. Since the transmission gate switch exhibits a resistance when they are turned on (i.e. in track mode), this circuit can be reduced to a simple RC network. [34 uses this simplification to develop the on resistance of the transmission gate as shown in Equation F.23.

$$
R_{\mathrm{on}}=\frac{1}{\mu_{\mathrm{n}} C_{\mathrm{ox}} \frac{W}{L}\left(V_{\mathrm{gs}}-V_{\mathrm{t}}\right)}
$$

Assuming the voltage applied to the gate of the transmission gate remains constant, the varying value of the signal being sampled causes $\mathrm{V}_{\mathrm{gs}}$ to vary. Also, the threshold voltage of the transmission gate will vary with the signal being sampled because $V_{t}$ is a 
function of the source-body voltage, $\mathrm{V}_{\mathrm{sb}}$ as shown in Equation F.24.

$$
V_{t}=V_{t o}+\gamma\left[\sqrt{2\left|\phi_{f}\right|+V_{s b}}-\sqrt{2\left|\phi_{f}\right|}\right]
$$

These two factors result in a variation in the transmission gate's on resistance. This variation results in a distortion of the sampled signal. 


\section{Bibliography}

[1] F. Chen, A. Chandrakasan, and V. Stojanovic. A Low-power Area-efficient Switching Scheme for Charge-sharing DACs in SAR ADCs. IEEE Custom Integrated Circuits Confrence, 2010.

[2] Buzsaki G. Rhythms of the brain. Oxford University Press, ISBN 0-19-530106-4, 2006.

[3] K Abdelhalim. Wireless Neural Recording and Stimulation SOCs for Monitoring and Treatment of Intractable Epilepsy. PhD thesis, University of Toronto, 2013.

[4] M. S. Chae, W. Liu, and M. Sivaprakasam. Design Optimization for Integrated Neural Recording Systems. IEEE Journal of Solid-State Circuits, 2008.

[5] R. Harrison, P. Watkins, R. Kier, R. Lovejoy, D. Black, B. Greger, and F. Solzbacher. A Low-power Integrated Circuit for a Wireless 100-electrode Neural Recodring System. IEEE Journal of Solid-State Circuits, 2007.

[6] M. Chae, W. Liu, Z. Yang, T. Chen, J. Kim, M. Sivaprakasam, and M. Yuce. A 128-Channel 6mW Wireless Neural Recording IC with On-the-Fly Spike Sorting and UWB Transmitter. ISSCC, 2008.

[7] Karim Abdelhalim. Ultra Low Power ADC for Bioedical Applicaitons. Master's thesis, Carleton University, June 2007.

[8] J. McCreary and P. Grey. All-MOS charge redistribution analog-to-digital conversion techniques. I,. IEEE Journal of Solid State Circuits, 1975. 
[9] R. Suarez, P. Grey, and D. Hodges. All-MOS charge-redistribution analog-to-digital conversion techniques. II,. IEEE Journal of Solid-State Circuits, 1975.

[10] R. Jacob Baker. CMOS Circuit Design, Layout, and Simulation. IEEE Press, 3rd edition, 2010.

[11] Shi Bu and Ka Nag Leung. A 116-dB CMOS op amp with repetitive gain boosting and subthreshold operation. Electron Devices and Solid-State Circuits (EDSSC), 2013 IEEE International Confrence of.

[12] Tony Chan Carusone, David Johns, and Ken Martin. Analog Integrated Circuit Design. John Wiley and Sons, second edition, 2012.

[13] W. Xu and E.G. Friedman. Clock Feedthrough in CMOS analog Transmission Gate Switches. ASIC/SOC Confrence, 2002. 15th Annual IEEE International, 2002.

[14] G. Van derPlas and B. Verbruggen. A $150 \mathrm{MS} / \mathrm{s} 133 \mu \mathrm{W} 7$ bit ADC in 90nm Digital CMOS. Solid-State Circuits, IEEE Journal of, vol. 43, December 2008.

[15] p. Nuzzo, C. Nani, C. Armiento, A. Sangiovanni-Vincentelli, J. Craninckx, and G. Van derPlas. A 6-bit 50MS/s $150 \mathrm{MS} / \mathrm{s}$ Threshold Configuring SAR ADC in 90nm Digital CMOS. Circuits and Systems I: Regular Papers, IEEE Transactions on, no. 99, 2011.

[16] P. Nuzzo, G. Van der Plas, F. De Bernardinis, L. Van der Perre, B. Gyselinckx, and P. Terreni. A $10.6 \mathrm{~mW} / 0.8 \mathrm{pJ}$ Power-Scalable $1 \mathrm{GS} / \mathrm{s} 4 \mathrm{~b}$ ADC in 0.18um CMOS with 5.8GHz ERBW. Design Automation Confrence, January 2006.

[17] A. Nikoozadeh and B. Murmann. An Analysis of Latch Comparator Offset Due to Load Capacitor Mismatch. Circuits and Systems II: Express Briefs, IEEE Transactions on, volume 53, December 2006.

[18] Alan Hastings. An Introduction to Matching and Layout. http://www2.engr. arizona.edu/ brew/ece304spr07/PowerPoint/A_Hastings (4_4_05) [1] .ppt. 
[19] N. H. E. Weste and D. M. Harris. CMOS VLSI design: a circuits and systems perspective. Addison-Wesley, first edition, 2010.

[20] S.M. Kang and Y. Leblebici. CMOS Digital Integrated Circuits: Analysis and Design. McGraw-Hill, first edition, 2003.

[21] B. N. Rabaey and A.P. Chandrakasan. Digital Integrated Circuits: A Design Perspective. Pearson Education, first edition, 2003.

[22] Clifford E Cummings. The Fundamentals of Efficient Synthesizable Finite State Machine Design using NC-Verilog and BuildGates. International Cadence Usergroup Confrence, September 16-18 2002.

[23] B. Cherkauer and E. Friedman. Unification of speed, power, area, and reliability in CMOS tapered buffer design. ISCAS '94 Circuits and Systems, 1994 IEEE International Symposium on, vol. 4, pp. 111 - 114 vol. 4, May 1994.

[24] IBM Microelectronics Division. IBM Foundry and Manufacturing Services Education, CMOS8RF V1.2.0.0 Design Kit Training . March 2006.

[25] Texas Instruments. Specification and architectures of sample-and-hold amplifiers. Texas Instruments Literature Number: SNOA223, July 1992.

[26] IEEE std. 1241-2000. IEEE Standard for Terminology and Test Methods for Analogto-Digital Converters. IEEE, ISBN 0-7381-2724-8 2001.

[27] W. Kester. The Data Conversion Handbook. New York: Elsevier, 2005.

[28] Behnaz Mortazavi. Modeling Leakage in Sub-Micron CMOS Technologies. Master's thesis, Simon Frazer University, December 2004.

[29] R. Inagnki, M. Miura-Mattausch, and Y. Inoue. A GIDL-Current Model for Advanced MOSFET Technologies Implemented into HiSIM2. Communications, Circuits and Systems, 2007. ICCCAS 2007 International Confrence on, 11-13 July $200 \%$. 
[30] K. Roy, S. Mukhopadhyay, and H. Mahmoodi-Meimand. Leakage Current Mechanisms and Leakage Reduction Techniques in Deep-Submicrometer CMOS Circuits. Proceedings of the IEEE, 2003.

[31] G. Wegmann, E. Vittoz, and F. Rahali. Charge injection in analog MOS switches. IEEE Jounral of Solid-State Circuits, volume SC-22, number 6, December 1987.

[32] J.H. Shieh, M. Patil, and B.J. Sheu. Modeling charge injection in MOS analog switches. IEEE Transactions on Circuits and Systems, volume CAS-34, February 1987.

[33] Behzad Razavi. Principles of Data Conversion System Design. IEEE Press, first edition, 2000.

[34] A. Ong, V.I. Prodanov, and M. Tarsia. A Method for Reducing the Varation in "On" Resistance of a MOS Sampling Switch. Circuits and Systems, 2000. Proceedings. ISCAS 2000 Geneva., The 2000 IEEE International Symposium on, Volume 5, 2000. 